\title{
The evaluation of a school-based military style high-intensity interval training intervention on adolescent's physical health, mental well-being and quality of life
}

\author{
Eddolls, William T.B.
}

How to cite:

Eddolls, William T.B. (2018) The evaluation of a school-based military style high-intensity interval training intervention on adolescent's physical health, mental well-being and quality of life. Doctoral thesis, Swansea University.

http://cronfa.swan.ac.uk/Record/cronfa48137

Use policy:

This item is brought to you by Swansea University. Any person downloading material is agreeing to abide by the terms of the repository licence: copies of full text items may be used or reproduced in any format or medium, without prior permission for personal research or study, educational or non-commercial purposes only. The copyright for any work remains with the original author unless otherwise specified. The full-text must not be sold in any format or medium without the formal permission of the copyright holder. Permission for multiple reproductions should be obtained from the original author.

Authors are personally responsible for adhering to copyright and publisher restrictions when uploading content to the repository.

Please link to the metadata record in the Swansea University repository, Cronfa (link given in the citation reference above.) 


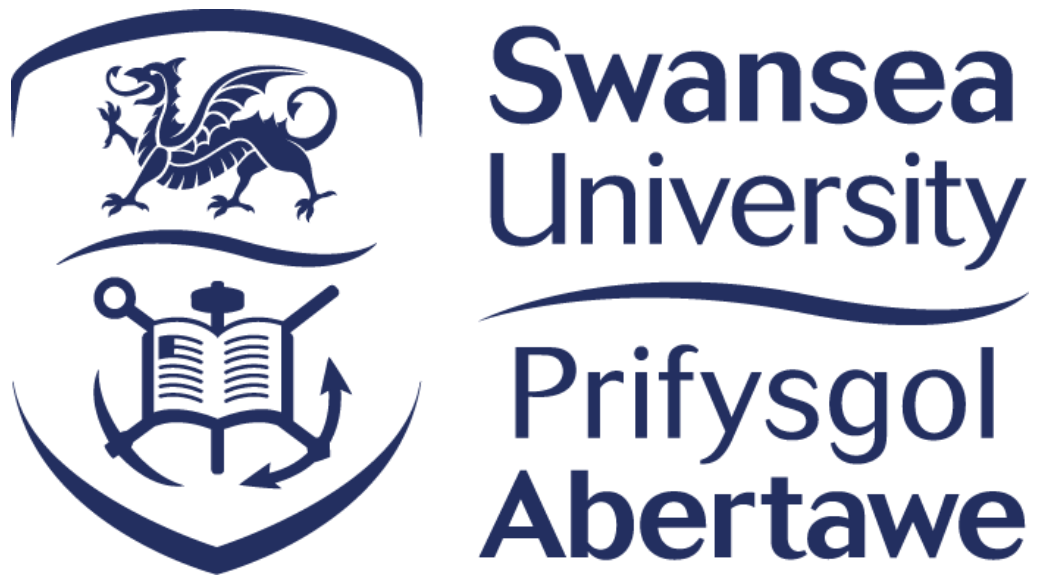

The evaluation of a school-based military style high-intensity interval training intervention on adolescent's physical health, mental well-being and quality of life

William Thompson Bezant Eddolls BSc (Hons)

Submitted to Swansea University in fulfilment of the requirements for the Degree of Doctor of Philosophy

Swansea University

2018 


\begin{abstract}
Reversing the decline in adolescents' mental health has been identified as a priority, especially considering its links to quality of life (QoL). Given that physical activity and exercise have been suggested as potential methods to ameliorate poor mental health and QoL, high-intensity interval training (HIIT) has been suggested as a timeeffective means to improve conditions such as anxiety and depression, as well as QoL. Therefore, in collaboration with Commando Joe's ${ }^{\circledR}$, the aim of this thesis was to design, conduct and evaluate the impact of a HIIT intervention on adolescents' mental health and QoL.

Prior to the design element of this thesis, Study 1 investigated and confirmed a significant relationship between vigorous physical activity, the unstructured form of HIIT, physiological and psychological health and QoL. Subsequently, utilising amalgamated findings from previous formative research and the systematic review included within Chapter 3, a HIIT intervention was designed and conducted. Study 2 therefore examined the effect of the 6-month HIIT intervention on body mass index, physical activity, cardiorespiratory fitness, anxiety, depression and QoL in adolescents, however, no significant changes to mental well-being, physiological health or QoL were exhibited. Consequently, to identify areas for improvement for future research, Study 3 evaluated the HIIT intervention using thematically analysed focus-groups presented by pen profiles. Results indicated that HIIT was an enjoyable form of exercise for adolescents, however, to maximise participation, factors related to session timing, exercise activities, group composition, instructor and exercise progression and protocol should be accounted for.

Overall, while HIIT did not improve mental health or QoL, no detrimental effects were noted. Advancing previous research, the present thesis provides support that HIIT is an enjoyable, feasible and time-efficient form of exercise, although further research is warranted to establish whether it can elicit improvements to mental health or QoL.
\end{abstract}




\section{Declarations and Statements}

This work has not previously been accepted in substance for any degree and is not being concurrently submitted in candidature for any degree.

Signed:

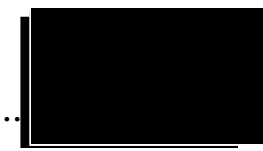
(candidate)

Date: $14 / 03 / 2018$

\section{STATEMENT 1}

This thesis is the result of my own investigations, except where otherwise stated. Other sources are acknowledged by footnotes giving explicit references. A bibliography is appended.

Signed:

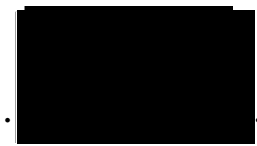
(candidate)

Date: $14 / 03 / 2018$

\section{STATEMENT 2}

I hereby give consent for my thesis, if accepted, to be available for photocopying and for inter-library loan, and for the title and summary to be made available to outside organisations.

Signed:

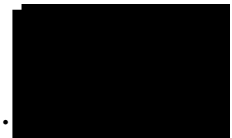
(candidate)

Date: $14 / 03 / 2018$ 


\section{Contents Page}

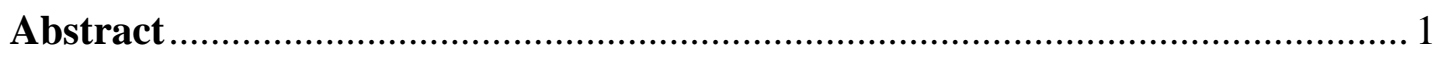

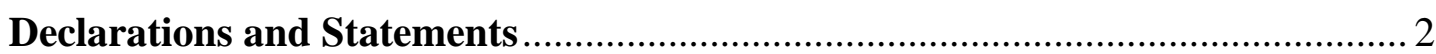

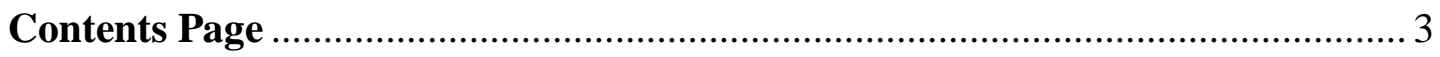

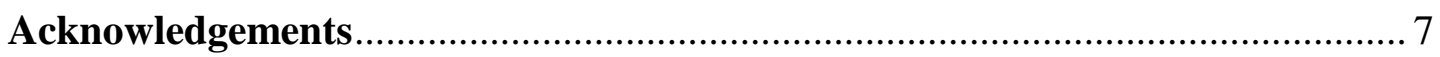

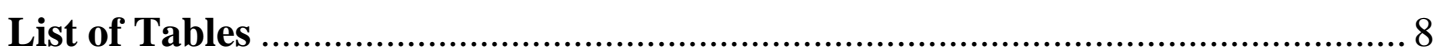

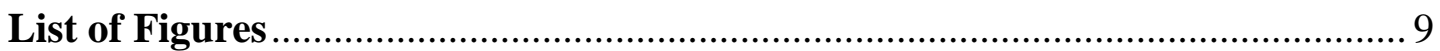

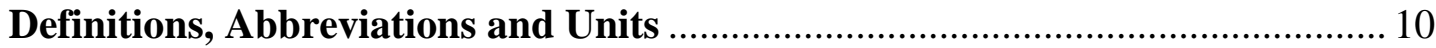

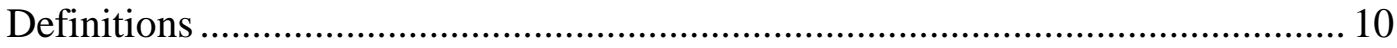

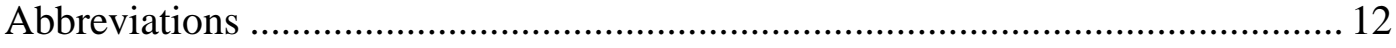

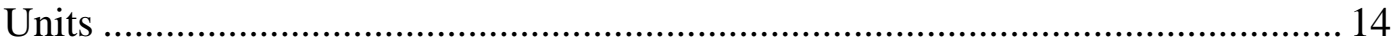

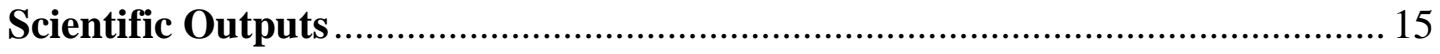

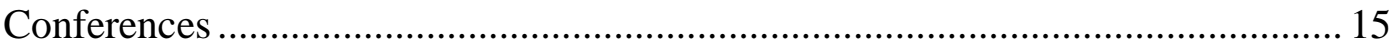

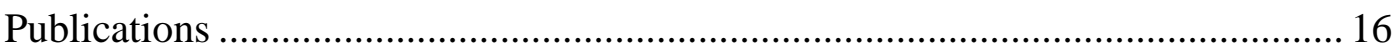

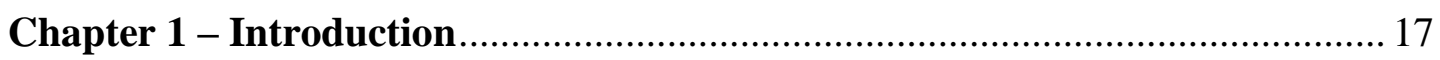

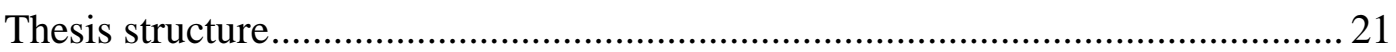

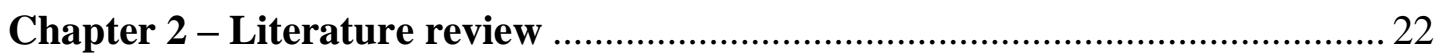

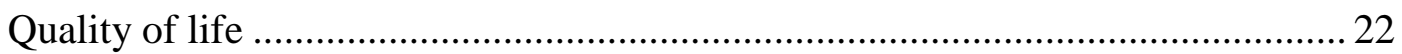

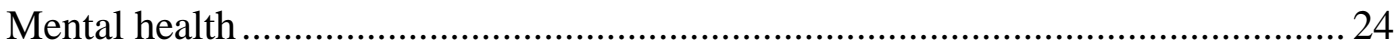

Measuring QoL and mental health in children and adolescents ........................... 24

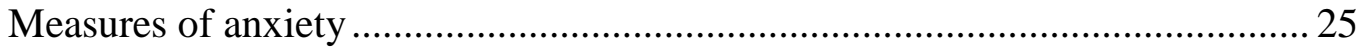

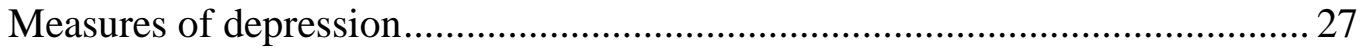

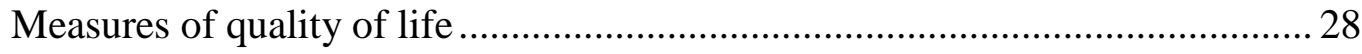

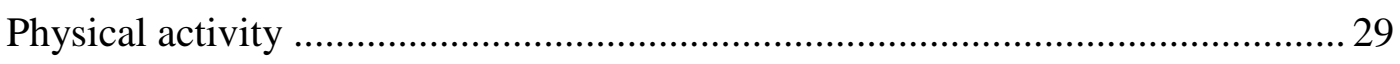

Effects of physical activity on mental health ................................................. 30

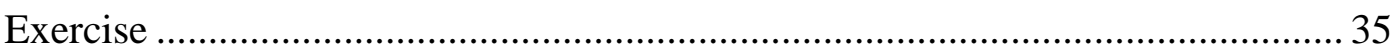


Dose-response of physical activity and exercise interventions on mental health .. 39

Anxiety.

Depression

Further considerations of a dose-response in relation to physical activity and exercise

Mechanistic basis of physical activity and exercise on mental health .45

High-intensity interval training .46

Effects of high-intensity interval training on mental health .48

Overall summary 50

Chapter 3 - Effects of high-intensity interval training on physiological health in children and adolescents.

Introduction .52

Methodology 54

Inclusion criteria .54

Results 56

Risk of bias 62

Body mass and composition 62

Cardiovascular health 64

Discussion .69

Conclusion .71

Thesis study map 73

Chapter 4 - The association between physical activity, fitness and body size and their effect on mental well-being and quality of life in children and adolescents (Study 1) .74

Introduction .74

Methods .76

Participants .76 
Measures .76

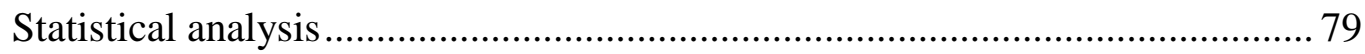

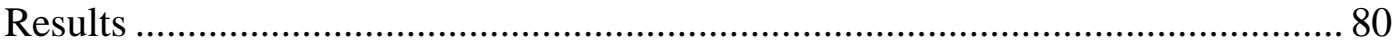

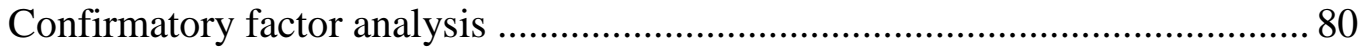

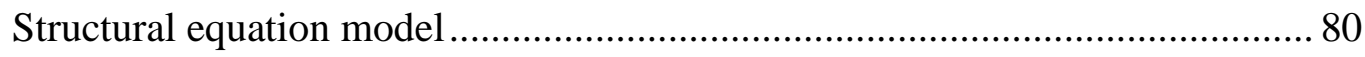

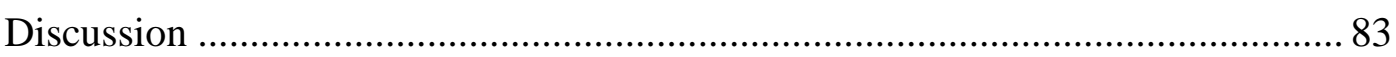

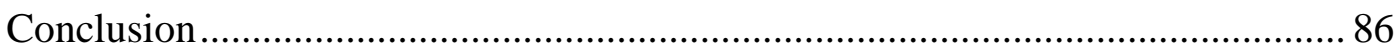

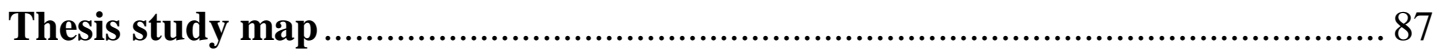

Chapter 5 - The effect of high-intensity interval training on mental well-being and quality of life in adolescents: the eXercise for Asthma with Commando Joe's ${ }^{\circledR}$

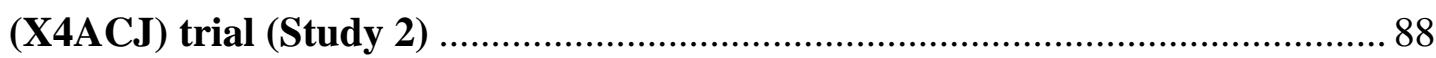

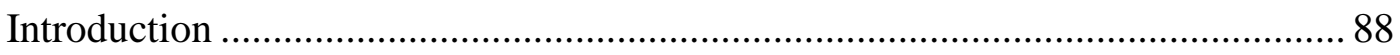

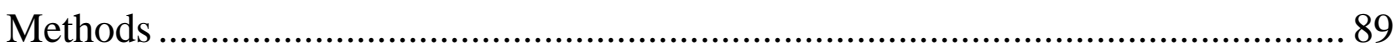

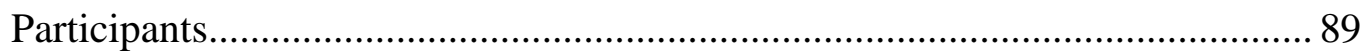

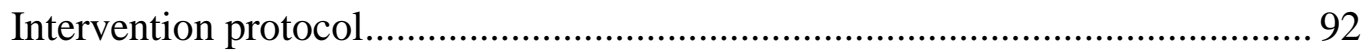

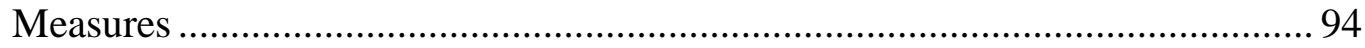

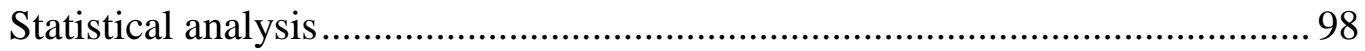

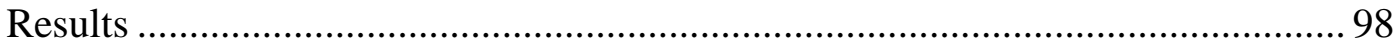

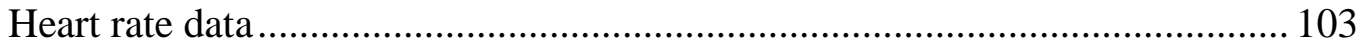

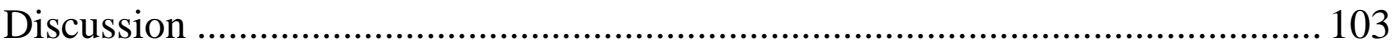

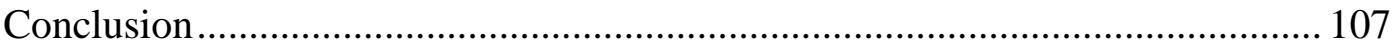

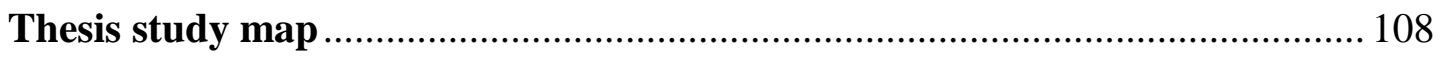

Chapter 6 - Exploring adolescent perceptions of a high-intensity interval training intervention: evaluating the eXercise for Asthma with Commando Joe's ${ }^{\circledR}$

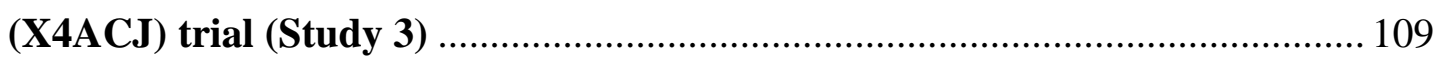

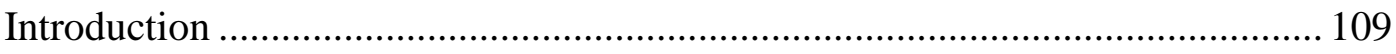

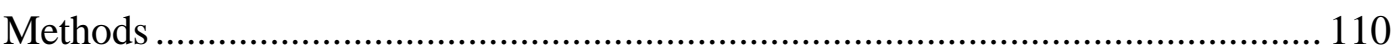

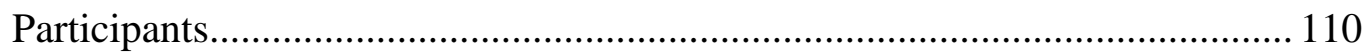




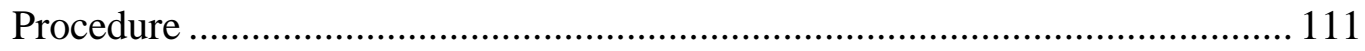

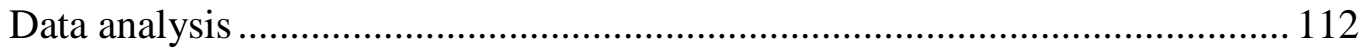

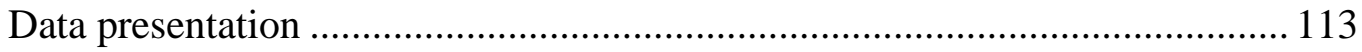

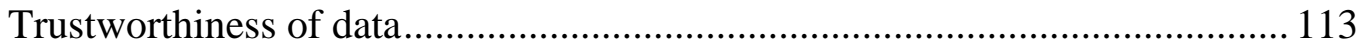

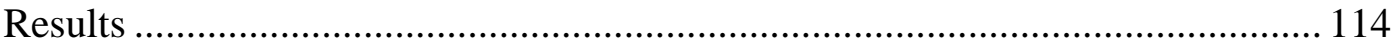

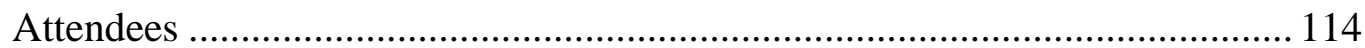

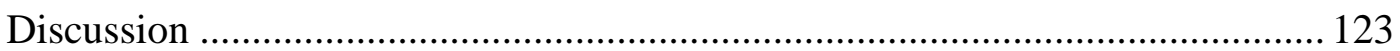

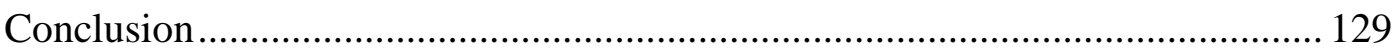

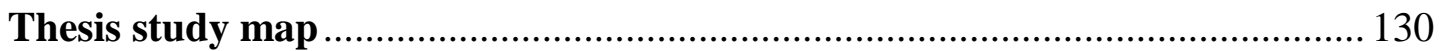

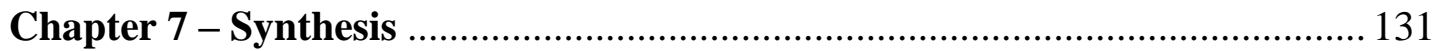

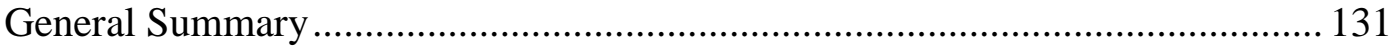

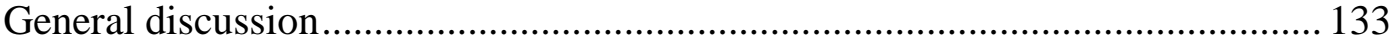

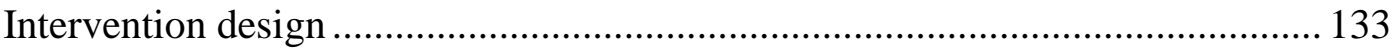

Effects of VPA and HIIT on mental health and QoL ....................................... 136

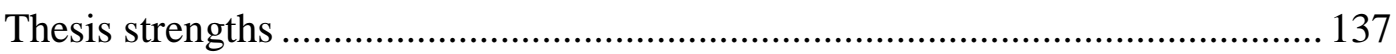

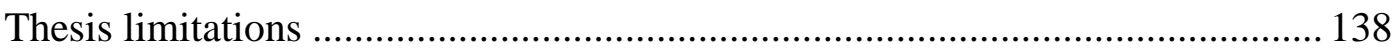

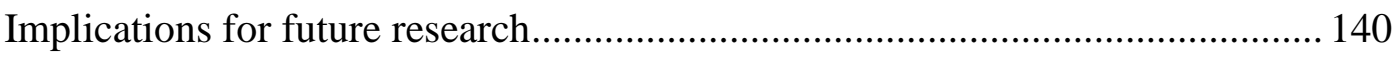

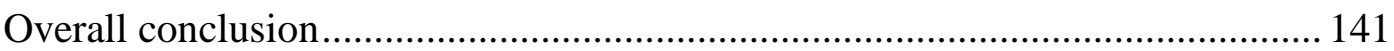

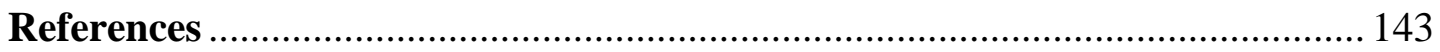

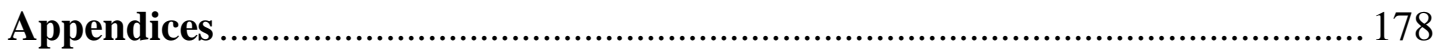




\section{Acknowledgements}

I would like to sincerely thank my supervisory team, Dr Kelly Mackintosh, Dr Melitta McNarry and Professor Gareth Stratton, firstly for the opportunity they gave me but also for their support and expert guidance throughout my $\mathrm{PhD}$. I would also like to thank my research team, Dr Charles Winn and Mr Nicholas Wade, for your invaluable contributions. Furthermore, I would also like to extend my gratitude to all of those that helped with data collection, there are too many to name, but without you it would have been impossible. Also, I must thank Dr Leanne Lester for her excellent advice during my $\mathrm{PhD}$.

The work in this thesis was primarily funded by Commando Joe's Fun and Fitness Limited. As such, I would like to thank the organisation, specifically Mr Michael Hamilton, Mr Stephen Hodson and Mr Matthew Hudson. Also, to the schools and teachers that supported the project and to every pupil involved, especially those that took part in the intervention, thank you.

Finally, but most importantly, thank you to my family, I couldn't have done it without you. Mum and Dad, thank you for giving me the push I needed to start this $\mathrm{PhD}$, and thank you for supporting me throughout. Harry, thank you for providing me with the competition and subsequent motivation to complete this $\mathrm{PhD}$ - bettering you has spurred me on. And of course, Maddy, thank you for your unwavering love and support through the entirety of my $\mathrm{PhD}$. When my confidence dropped, you were there to pick me up again and provide me with the belief I needed to push through. 


\section{List of Tables}

Table 1.1 Aims of the experimental chapter studies within this thesis .................... 21

Table 3.1 Characteristics of studies examining pre-pubertal participants.................58

Table 3.2 Characteristics of studies examining pubertal participants ......................60

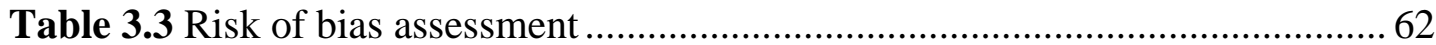

Table 3.4 Baseline and post-intervention changes to body mass/composition and effect size between HIIT and control/moderate protocols

Table 3.5 Baseline to post-intervention changes in SBP/DBP and effect size between HIIT and control/moderate protocols 65

Table 3.6 Baseline and post-intervention changes in cardiovascular disease biomarkers and effect size between HIIT and control/moderate protocols. 68

Table 4.1 Means and SD among of the measured variables of the study participants, dichotomised by sex

Table 4.2 Standardised and unstandardised regression weights with standard errors

Table 5.1 Examples of exercise/rest intervals and activities undertaken during those sessions .92

Table 5.2 Detailed examples of exercises .93

Table 5.3 Overview of trial timeline 95

Table 5.4 Anthropometric and maturational data at baseline 96

Table 5.5 The number of participants for which data was obtained at each time point 100

Table 5.6 Intervention and control group BMI, cardiorespiratory fitness, MVPA, anxiety, depression and quality of life mean scores and standard deviation over time

Table 6.1 Example interview questions 


\section{List of Figures}

Figure 3.1 Phases of study selection during data collection

Figure 4.1 Structural equation model (SEM) of physical activity levels, physiological health and their effect on mental well-being and quality of life in children and adolescents with standardised beta coefficients $(* p<0.05, * * p<0.01)$

Figure 5.1 Flow chart outlining the school recruitment process, group allocation including the number and percentage of pupils who consented within the school and intervention completion rate and matched controls as number and percentage of pupils who consented.

Figure 6.1 Attendee facilitators - pen profile of perceptions of a 6-month highintensity interval training intervention. $\mathrm{B}=\mathrm{boy}, \mathrm{G}=\mathrm{girl}$, $+\mathrm{ve}=$ positive 115

Figure 6.2 Attendee barriers - pen profile of perceptions of a 6-month high-intensity interval training intervention. $\mathrm{B}=$ boy, $\mathrm{G}=\operatorname{girl}$

Figure 6.3 Non-attendee facilitators - pen profile of perceptions of a 6-month highintensity interval training intervention. $\mathrm{B}=$ boy, $\mathrm{G}=$ girl, +ve $=$ positive

Figure 6.4 Non-attendee barriers - pen profile of perceptions of a 6-month highintensity interval training intervention. $\mathrm{B}=\mathrm{boy}, \mathrm{G}=$ girl, $-\mathrm{ve}=$ negative 


\section{Definitions, Abbreviations and Units}

\section{Definitions}

Adolescents

A transitional stage of physical and psychological development that generally occurs between the chronological age range of 12 to 17 years

Children

The developmental stage prior to the onset of puberty that generally occurs between the chronological ages of 4 to 11 years

High-intensity

exercise

Moderate physical activity

Moderate-to-vigorous physical activity

Physical activity
Intense exercise that is greater than or equal to a peak oxygen uptake of $90 \%$, an intensity that is equal to or greater than $100 \%$ maximal aerobic speed and/or an exercise induced peak heart rate equal to or greater than $90 \%$

Habitual activity corresponding to an energy expenditure between 3 and 6 metabolic equivalents

Habitual activity corresponding to an expenditure equal to or greater than 3 metabolic equivalents

"Any bodily movement produced by skeletal muscles resulting in energy expenditure above resting" (Caspersen, Powell, \& Christenson, 1985) 
Vigorous physical activity

Exercise
Habitual activity corresponding to an energy expenditure between 6 and 9 metabolic equivalents

A sub-component of physical activity that is carried out to sustain or improve health 


\section{Abbreviations}

ANOVA analysis of variance

BF\% body fat percentage

BMI body mass index

BP blood pressure

CES-DC Center for Epidemiologic Studies Depression Scale for Children

CI confidence intervals

CON control group

CVD cardiovascular disease

DBP diastolic blood pressure

EM expectation maximisation

FFM fat free mass

GAD-7 Generalized Anxiety Disorder 7

HDL high-density lipoprotein

HIIT high-intensity interval training

HPA hypothalamic-pituitary-adrenal axis

HR heart rate

ISAK International Society for the Advancement of Kinanthropometry

LDL low-density lipoprotein

MAS maximal aerobic speed

MSFT multi-stage fitness test

PedsQL Pediatric Quality of Life Inventory

QoL quality of life

SBP systolic blood pressure 
SEM structural equation modelling

TC total cholesterol

TG triglycerides

UK United Kingdom

$\dot{V} \mathbf{O}_{2}$ oxygen uptake

VPA vigorous physical activity

WC waist circumference

WHO World Health Organisation 


\section{Units}

cm centimetres

Hz hertz

$\mathbf{I U} \cdot \mathbf{m l}^{-\mathbf{1}}$ international unit per millilitre

kg kilogram

$\mathbf{k g} \cdot \mathbf{m}^{-2}$ kilogram per metre squared

$\mathbf{l} \cdot \mathbf{m i n}^{-1}$ litre per minute

$\mathbf{m g} \cdot \mathbf{d l}^{\mathbf{1}}$ milligram per decilitre

$\mathbf{m g} \cdot \mathbf{1 0 0 \mathbf { m l } ^ { - 1 }}$ milligram per one-hundred millilitres

$\mathbf{m l} \cdot \mathbf{k g} \cdot \mathbf{m i n}^{-1}$ millilitre per kilogram per minute

$\mathbf{m l} \cdot \mathbf{k g}^{-0.75} \cdot \mathbf{m i n}^{-1}$ millilitre per kilogram to the power of 0.75 per minute

$\mathbf{m m H g}$ millimetre of mercury

$\mathbf{m m o l} \cdot \mathbf{l}^{-1}$ millimoles per litre

pmol $\cdot \mathbf{l}^{-1}$ picomole per litre 


\section{Scientific Outputs}

\section{Conferences}

Eddolls, W. T. B., McNarry, M. A., Stratton, G., \& Mackintosh, K. A. Parental influences on children's physical self-perceptions, body composition and physical activity levels. $5^{\text {th }}$ Annual Public Health Science: A National Conference Dedicated to New Research in UK Public Health, Cardiff, UK. November 2016. Poster presentation.

Eddolls, W. T. B., McNarry, M. A., Lester, L., Winn, C. O. N., Stratton, G., \& Mackintosh, K. A. The association between physical activity, fitness and body mass index on mental well-being and quality of life in adolescents. $1^{\text {st }}$ Annual Pan-Wales Postgraduate Conference in Sport and Exercise Sciences, Swansea, UK. April 2017. Oral presentation.

Eddolls, W. T. B., McNarry, M. A., Winn, C. O. N., Wade, N. D., \& Mackintosh, K. A. The effects of a 6-month school-based high-intensity interval training intervention on mental well-being and quality of life in adolescents. $22^{\text {nd }}$ Annual Congress of the European College of Sport Science (ECSS), MetropolisRuhr, GER. July 2017. Minioral presentation. 


\section{Publications}

Eddolls, W. T. B., McNarry, M. A., Stratton, G., \& Mackintosh, K. A. (2016). Parental influences on children's physical self-perceptions, body composition and physical activity levels. The Lancet, 388, S45.

Eddolls, W. T. B., McNarry, M. A., Stratton, G., Winn, C. O. N., \& Mackintosh, K. A. (2017). High-intensity interval training interventions in children and adolescents: a systematic review. Sports Medicine, 47(11), 2363-2374.

Winn, C. O. N., Mackintosh, K. A., Eddolls, W. T. B., Stratton, G., Wilson, A. M., Rance, J. Y., Doull, I. J. M., McNarry, M. A., Davies, G. A. (2017). Perceptions of asthma and exercise in adolescents with and without asthma. Journal of Asthma.

Eddolls, W. T. B., McNarry, M. A., Lester, L., Winn, C. O. N., Stratton, G., \& Mackintosh, K. A. (2018). The association between physical activity, fitness and body mass index on mental well-being and quality of life in adolescents. Quality of Life Research. 


\section{Chapter 1 - Introduction}

Health is fundamental in allowing an individual to adapt to changing situations and self-manage (Huber et al., 2011) and is defined as "a state of complete physical, mental, and social well-being and not merely the absence of disease or infirmity" (World Health Organization, WHO, 2006, p. 1). Despite an increased awareness of the factors that can influence health, in recent years the prevalence of poor mental and physical health has increased in both child and adult populations (A. J. Cohen et al., 2017; Ng et al., 2014; Vos et al., 2015).

Mental health is defined as "a state of well-being in which every individual realizes his or her own potential, can cope with the normal stresses of life, can work productively and fruitfully, and is able to make a contribution to her or his community" (WHO, 2004, p. 1). Whilst often overlooked in comparison to physical health, a recent report has suggested that mental health problems, such as anxiety and mood disorders, account for a large proportion of the overall global disease burden (Vos et al., 2015). Indeed, within younger populations, according to the WHO (2003) $20 \%$ of adolescents will experience a mental health problem. Furthermore, adolescents reporting recurrent sensations of anxiety or depression have doubled between the mid 1980's and mid 2000's (Collishaw, Maughan, Natarajan, \& Pickles, 2010), with one-in-ten 5-16 year olds in the UK diagnosed with a clinical mental health disorder (Green, McGinnity, Meltzer, Ford, \& Goodman, 2004). Specifically in Wales, a recent report stated that that the total number of referrals to the Child and Adolescent Mental Health Service has doubled between 2010 and 2014 (National Assembly for Wales Children Young People and Education Committee, 2014). Whilst mental health illnesses have conventionally only been associated with further mental health issues, there is growing evidence that they can also significantly contribute to physical ailments. For example, depression, the most prevalent mental health issue (National Collaborating Centre for Mental Health, 2011), has been directly linked to heart disease (Ferrari et al., 2013). Conversely, it has also been suggested that physical diseases, such as obesity, are also positively associated with mental health issues (Luppino et al., 2010; Scott et al., 2008).

In recent years, multiple studies have suggested that obesity in children has reached unparalleled levels (Abarca-Gómez et al., 2017; Lobstein et al., 2015; Ng et al., 2014), 
with statistics purporting that $\sim 30 \%$ of children are overweight or obese ( $\mathrm{Ng}$ et al., 2014; Ryley, 2013; Townsend et al., 2013). Furthermore, whilst reports have indicated a plateau in paediatric obesity over the last decade (Abarca-Gómez et al., 2017; Rokholm, Baker, \& Sørensen, 2010), this obesity epidemic still represents a significant burden on the National Health Service (NHS). Specifically, current estimates indicate that overweight and obesity, and their deleterious health consequences such as cancer (Calle \& Thun, 2004), type 2 diabetes mellitus (American Diabetes Association, 2000) and coronary heart disease (American Heart Association, 1997), cost the NHS £5.1 billion per year (Scarborough et al., 2011).

Impaired physical and mental health can significantly affect quality of life (QoL), which is a fundamental measure in the evaluation of population health (Helseth \& Misvær, 2010), defined by the WHO (2006) to include a person's perception of their own physical, mental and social well-being. Cardiorespiratory fitness and body mass index (BMI) have both been shown to influence QoL, as those with a greater level of cardiorespiratory fitness experience a better QoL (J. R. Andersen et al., 2017; Morales et al., 2013; Sloan, Sawada, Martin, Church, \& Blair, 2009) and overweight or obesity often indicative of a poorer QoL (Swallen, Reither, Haas, \& Meier, 2005; J. Williams, Wake, Hesketh, Maher, \& Waters, 2005). Additionally, mental well-being has also been shown to partially determine QoL, with those suffering from mental health issues at greater risk of a poor QoL (Sharpe et al., 2016; Wafa et al., 2016). Accordingly, in line with mental and physical health, recent findings have suggested that self-reported QoL has significantly declined in a number of populations over the last decade (Cui \& Zack, 2013; Zack, 2013).

Causes of poor mental and physical health are multifaceted; in addition to factors such as diet (Kuźbicka \& Rachoń, 2013; Sahoo et al., 2015) and daily stresses (Schönfeld, Brailovskaia, Bieda, Zhang, \& Margraf, 2016), physical activity, or lack thereof, and poor cardiorespiratory fitness have been extensively linked to adverse health (Bize, Johnson, \& Plotnikoff, 2007; Freedman, Mei, Srinivasan, Berenson, \& Dietz, 2007; Janssen et al., 2005; Kopelman, 2000; Monyeki, Neetens, Moss, \& Twisk, 2012; Penedo \& Dahn, 2005; Ruggero, Petrie, Sheinbein, Greenleaf, \& Martin, 2015; Trost, Kerr, Ward, \& Pate, 2001). Although the importance of physical activity is apparent, research has shown that large proportions of children are not physically active. Specifically, for children aged between 5 and 15 years in England, only 23\% of boys 
and $20 \%$ of girls meet UK physical activity guidelines of at least 60 min of moderateto-vigorous physical activity every day (Scholes, 2016). Furthermore, research has suggested that adolescent cardiorespiratory fitness, albeit within England, is in decline (Sandercock, Ogunleye, \& Voss, 2015; Sandercock, Voss, McConnell, \& Rayner, 2010; Stratton et al., 2007), with the cardiorespiratory fitness of young people in Wales among the lowest in Europe (Tomkinson \& Olds, 2007), though, this is likely related to low physical activity (Parikh \& Stratton, 2011), Considering that adolescence is a key stage for the development of health-related behaviours (Dumith, Gigante, Domingues, \& Kohl, 2011), with poor health tracking into later life (Freedman et al., 2007), the design, implementation and evaluation of effective interventions targeted at increasing youth physical activity levels and cardiorespiratory fitness are imperative.

Given that children and adolescents spend a considerable proportion of their waking hours in school (Fox, Cooper, \& McKenna, 2004), schools been extensively utilised as a foundation for the implementation of physical activity and exercise interventions (Christian et al., 2016; Mackintosh, Niezen, \& Eslambolchilar, 2016; K. L. Weston et al., 2016). Additionally, interventions within schools have the ability to reach a large number of children from diverse socio-economic backgrounds (Fox et al., 2004). Moreover, given that schools have the personnel, such as physical education teachers, that can deliver effective physical activity and exercise interventions, the possible sustainability of such interventions is increased (Hoelscher et al., 2004; Saunders et al., 2012). Indeed, the design of interventions that can be implemented within a "real world" setting are recommended (De Bourdeaudhuij et al., 2011) as they are less costly (Warren, Henry, Lightowler, Bradshaw, \& Perwaiz, 2003), can be integrated within the curriculum and are have a greater chance of being maintained. However, whilst traditional school-based physical activity, such as physical education lessons, should be encouraged, it has been suggested that these alone are unlikely to afford adequate physical activity to elicit health benefits (Biddle, Gorely, \& Stensel, 2004). Indeed, given the decreasing hours devoted to physical education within the school curriculum (Rumsby, 2015), it could be postulated that additional complementary exercise, the structured form of physical activity, is required.

Whilst traditional exercise interventions have primarily used high-volume moderateintensity continuous exercise (Hansen, Froberg, Hyldebrandt, \& Nielsen, 1991; McMurray et al., 2002; Meyer, Kundt, Lenschow, Schuff-Werner, \& Kienast, 2006), 
the relevance of such exercise to the sporadic high-intensity nature of children's habitual play patterns has been challenged (Bailey et al., 1995). Additionally, highvolume moderate-intensity continuous exercise can often be time-consuming, a key factor commonly cited as a barrier to exercise (Donahue, Mielenz, Sloane, Callahan, \& Devellis, 2006; Lovell, Ansari, \& Parker, 2010; Reichert, Barros, Domingues, \& Hallal, 2007; Welch, McNaughton, Hunter, Hume, \& Crawford, 2009). Consequently, high-intensity interval-based exercise or high-intensity interval training (HIIT) has recently been identified as a potentially effective and time-efficient form of exercise and health promotion (Costigan, Eather, Plotnikoff, Hillman, \& Lubans, 2016; Gibala, 2007). Indeed, given that vigorous physical activity has been associated with improved health (Braithwaite et al., 2017; Drenowatz, Prasad, Hand, Shook, \& Blair, 2016; Gerber et al., 2014; Kenchaiah, Sesso, \& Gaziano, 2009; Parfitt, Pavey, \& Rowlands, 2009), exercise, could provide a necessary mediatory step to engender positive longterm behavioural change.

Whilst there has been substantial development regarding the assessment of highintensity interval-based exercise on physiological health in adolescents (Boddy, Stratton, Hackett, \& George, 2010; Lambrick, Westrupp, Kaufmann, Stoner, \& Faulkner, 2016; Tjønna et al., 2009; K. L. Weston et al., 2016). This could, at least in part, be attributed to the varied success at eliciting significant improvements (Baquet et al., 2002; Lau et al., 2014; Rosenkranz, Rosenkranz, Hastmann, \& Harms, 2012), potentially owing to ineffective protocols. Indeed, to date, there has been no research that has provided information as to the most effective HIIT protocol for adolescents. More pressing, however, given the considerable progress regarding the effect of HIIT on adolescent physiological health, there is a stark paucity of research focusing on mental well-being and/or QoL (Logan, Harris, Duncan, \& Schofield, 2014; McCrone, Dhanasiri, Patel, Knapp, \& Lawton-Smith, 2008). With the exception of Shepherd et al. (2015) in adults, and Costigan, Eather, Plotnikoff, Hillman and Lubans (2016) in adolescents, little research is available regarding the influence of HIIT on mental health and/or QoL. Furthermore, although some effort has been made to ascertain adolescents' post-intervention perceptions of HIIT (Buchan et al., 2013; Costigan et al., 2017), the findings of both studies were embedded alongside physiological measures within main trial papers and, therefore, are limited in detail and indeed interpretation. As such, not only is there a need for further investigation, but a need for 
an in-depth evaluation as to participant's perceptions of HIIT, specifically regarding barriers and facilitators to participation. Therefore, the aim of this thesis was to design, conduct and evaluate a long-term HIIT intervention for on adolescents.

\section{Thesis structure}

This thesis contains seven chapters with the central theme addressing the effect of HIIT on mental well-being and QoL. An initial introduction detailing the justification for and the specific aims of this research are outlined within Chapter 1. A review of previous relevant literature is summarised within Chapter 2, primarily addressing the effects of physical activity, exercise and HIIT on mental health in children and adolescents. Within Chapter 3, a systematic review synthesising literature examining the effects of HIIT on physiological health in children and adolescents is presented, as well as recommendations concerning a HIIT protocol with regard to session structure, intensity, frequency and duration. Three experimental chapters are presented in Chapters 4 to 6 . A synthesis (Chapter 7) discussing the main findings of the results from the three experimental chapters concludes the thesis. The aims of the studies within the experimental chapters (Chapters 4 to 6) are outlined in Table 1.1.

Table 1.1 Aims of the experimental chapter studies within this thesis

\begin{tabular}{cl}
\hline Study & Aims \\
\hline $\mathbf{1}$ & To investigate the mediatory effects between vigorous physical \\
& activity, body mass index and cardiorespiratory fitness on \\
& depression, and their subsequent direct and indirect effects on QoL \\
& in children and adolescents. \\
& To assess the effects of a school-based HIIT intervention on anxiety, \\
& depression and QoL, in adolescents. \\
& Explore adolescent perceptions of a 6-month school-based HIIT \\
& intervention, addressing participants perceived barriers and \\
& facilitators to extra-curricular exercise participation.
\end{tabular}




\section{Chapter 2 - Literature review}

The first sections within this literature review provide an overview of quality of life (QoL) and mental health, the primary outcome measures within this thesis. Subsequently there is a discussion regarding the issues inherent to measuring mental health and QoL in children and adolescents, followed by a brief overview of measures of mental health and QoL. Also included within this literature review is a synopsis and critical analysis of key research relevant to physical activity, exercise and highintensity interval training (HIIT) and their influence on mental health and QoL. Furthermore, the dose-response effect of physical activity and exercise interventions on mental health as well as the mechanistic basis supporting the effects of physical activity and exercise on mental health are deliberated.

\section{Quality of life}

Since its introduction as a key term within medical literature midway through the twentieth century (Post, 2014), there has been a considerable number of attempts to define QoL, with varying outcomes (Cummins, 1997; de Wit \& Hajos, 2013; Kosher, Jiang, Ben-Arieh, \& Huebner, 2014; Ravens-Sieberer et al., 2006; Testa \& Simonson, 1996; World Health Organization, WHO; 2006). Given the lack of consensus, researchers have moved towards viewing QoL "as an organising concept that can be used for a number of purposes" (Schalock, 1996, p. 123). Indeed, it is now widely recognised that QoL is conceptualised and measured using three defined approaches, namely health-related QoL, social indicators and subjective well-being (Cummins, Lau, \& Stokes, 2004; Diener \& Suh, 1997; Michalos, 2004). However, whilst all the approaches are perhaps equally valid within their own purpose, health-related QoL, the concept used within this thesis, has become the most commonly utilised approach within in epidemiological and clinical health research (Wallander \& Koot, 2016). This approach measures an individual's subjective perceptions of their own health, disease, disability and impairment and is frequently used to capture the effects of health treatments and interventions (De Civita et al., 2005).

Since the WHO defined health as "a state of complete physical, mental, and social well-being and not merely the absence of disease or infirmity" (WHO, 2006, p. 1), QoL has been recognised as an integral component in the evaluation of a person's health status (Helseth \& Misvær, 2010) and as the fundamental target for health 
interventions (Ravens-Sieberer, Herdman, et al., 2014). Specifically, interventions or treatments implemented for the benefit of a person's health should ultimately improve QoL, or, at the very least, not be detrimental (Testa \& Simonson, 1996). As such, the utilisation of QoL as an outcome measure in clinical settings has substantially increased in recent years, particularly in children (S. A. Clarke \& Eiser, 2004). Specifically, there have been numerous comprehensive studies that have evaluated the QoL of children suffering from chronic illnesses, such as cancer (Vlachioti et al., 2016), asthma (Miadich, Everhart, Borschuk, Winter, \& Fiese, 2015) and cystic fibrosis (Koscik et al., 2005). Within these populations, independent of objective medical outcomes, QoL has emerged as an effective measure for the evaluation of treatments or interventions (Koot, 2001).

Despite the importance of QoL as a tool to measure a person's health (Helseth \& Misvær, 2010) and its emergence as an outcome measure within clinical populations, QoL's application as an intervention evaluation tool within the general population has been less extensive. Further, recent findings have suggested that QoL has significantly declined in adolescents over the last decade (Cui \& Zack, 2013), highlighting the need for interventions to specifically measure, and target, QoL. It could be postulated that such a paucity of research may be due to the potential difficulty in eliciting improvements given the subjective nature of QoL and the inability to directly enhance it (Zack, 2013). However, previous research has demonstrated that by modifying health behaviours associated with QoL, such as physical health and mental well-being, improvements can be indirectly elicited. For example, cardiorespiratory fitness and body mass index (BMI) have both been identified as predictors of QoL, with a higher cardiorespiratory fitness level associated with a better QoL (J. R. Andersen et al., 2017; Morales et al., 2013; Sloan et al., 2009), and those that are overweight or obese typically experiencing a poorer QoL (Swallen et al., 2005; J. Williams et al., 2005). Additionally, those suffering from mental health issues or not meeting physical activity guidelines have also been identified as being at greater risk of a poor QoL (Sharpe et al., 2016; Wafa et al., 2016). Despite some evidence supporting increased physical activity as a means to indirectly improve QoL (Gill et al., 2013), the paucity of research is stark, with no quality research in the general child and adolescent population available. Consequently, the influence of physical activity and/or exercise on health 
behaviours warrant further attention as potential mediators through which to enhance QoL.

\section{Mental health}

Mental health is an important, but often forgotten, aspect of health that encompasses a person's emotional, psychological and social well-being, and is included within the WHO constitutional definition of health (WHO, 2006). Despite this, mental health problems remain one of the leading causes of disease globally (Vos et al., 2015). Depression and anxiety, the most common mental health issues (National Collaborating Centre for Mental Health, 2011) can significantly contribute to deleterious health consequences such as cardiovascular disease and poor QoL (Ferrari et al., 2013). Previously, mental health treatments have included potentially harmful techniques, such as electroconvulsive therapy and lobotomies, however, medicine has since progressed towards treatments such as psychotherapy and pharmaceuticals. Nonetheless, such treatments are often expensive (McCrone et al., 2008) and timeconsuming (Gladstone, Beardslee, \& O'Connor, 2011), with the safety of pharmacological interventions remaining questionable (Giraldi, 2017). Furthermore, preventing mental health issues rather than treating the symptoms should be a priority, not least given the greater long-term effectiveness (Fisak, Richard, \& Mann, 2011). Therefore, although the sources of poor mental and physical health are multifaceted, low physical activity has been extensively associated with poor health (Bize et al., 2007; Freedman et al., 2007; Janssen et al., 2005; Kopelman, 2000; Penedo \& Dahn, 2005; Trost et al., 2001). Consequently, increasing physical activity levels has been suggested as a time-efficient and low-cost means to ameliorate poor mental health and QoL (J. R. Andersen et al., 2017; Biddle \& Asare, 2011; Wafa et al., 2016).

\section{Measuring QoL and mental health in children and adolescents}

Possibly due the inherent high cost of a health professional administering mental health instruments (Curtis, 2012), despite their limitations (Paulhus \& Vazire, 2005), selfreport questionnaires are perhaps the most commonly utilised method of collecting mental health and QoL data. In addition to concerns relating to the validity and reliability of self-reporting, issues pertaining to response reliability also exist. For example, within some research, especially in studies involving younger children, questionnaires are often completed externally via parental proxy-report. Subsequently, 
the reliability of the data collected is questionable given that it is unknown whether a child or adolescent and their parent share the same concepts (Ravens-Sieberer, Karow, Barthel, \& Klasen, 2014). Furthermore, it has been suggested that a tendency for children to base their responses to questions on their state of mind at that moment, rather than their general levels, may decrease the reliability of their answers (Costello, Dulcan, \& Kalas, 1985; Marsh, Debus, \& Bornholt, 2008)..

Given their importance, the number of tools that measure QoL and mental health issues are vast, with over a thousand methods of assessment available for QoL alone (Ubel, Loewenstein, \& Jepson, 2003). Although new and relatively sparse in comparison to adult measures (Ravens-Sieberer, Karow, et al., 2014), validated measures of children and adolescent mental health and QoL have rapidly multiplied in recent years (Solans et al., 2008; Zima et al., 2013). Prior to this, measuring mental health and/or QoL in children and adolescents was largely restricted. Specifically in relation to QoL, it has been suggested that both children and adolescents develop their own concept of the meaning of QoL, such as an increased appreciation for its social function (Drotar, 2004). Despite this, questionnaires were initially transferred from adult to child populations ad hoc, and without adequate validation (Ravens-Sieberer, Karow, et al., 2014). Whilst also applicable to mental health measures, given that QoL measures within younger populations should recognise cognitive development, reading ability and emotional maturity (Eiser, Mohay, \& Morse, 2000; M. Hill, 1997; Matza, Swensen, Flood, Secnik, \& Leidy, 2004), early ad hoc measures possibly confounded such issues. However, despite these limitations, it has been suggested that if participant maturity and cognitive development is accounted for with respect to question presentation and reading level, self-reporting questionnaires can yield valid and reliable results (Riley, 2004; Schmidt, Garratt, \& Fitzpatrick, 2000; Truman et al., 2003).

\section{Measures of anxiety}

Commonly used measures of anxiety symptoms within clinical practice and research include the Beck Anxiety Inventory (BAI; Beck, Epstein, Brown, \& Steer, 1988), the Generalised Anxiety Disorder 7 (GAD-7) assessment (Spitzer, Kroenke, Williams, \& Löwe, 2006) and the State Trait Anxiety Inventory - Children (STAI-C; Spielberger \& Edwards, 1973). The BAI is a 21 -item self-reporting measure of anxiety symptoms that focuses on somatic symptoms, such as dizziness or increased heart rate (HR). 
Specifically, the BAI is easily administered and scored, has strong validity and reliability within adult populations (Julian, 2011) and has acceptable psychometric properties in adolescents (Osman et al., 2002). Despite this, however, like the BDI, the BAI is copyrighted and therefore also requires a user fee. Furthermore, the BAI does not assess additional leading symptoms of anxiety (i.e., worry) and other cognitive aspects of anxiety and, therefore, subsequently limits its interpretation.

Identified as the best performing measure of generalised anxiety disorder when compared to other measures (Herr, Williams, Benjamin, \& McDuffie, 2014), the GAD-7 is a 7-item self-reporting questionnaire that is often used within a primary care setting (Löwe et al., 2008). Whilst short in length, the GAD-7 has been validated in adult (Löwe et al., 2008) and adolescent populations (Mossman et al., 2017) and, therefore, is an extremely quick, easy and efficient tool to assess symptoms of anxiety. However, although the GAD-7 may be used as a screening tool as part of an initial diagnostic evaluation, further evaluation by a trained mental health professional is required to fully diagnose anxiety. Furthermore, despite validation (Mossman et al., 2017) and some limited use (Wong, Kady, Mewton, Sunderland, \& Andrews, 2014), the GAD-7 has not been widely utilised within adolescent populations, and therefore, inter-study comparisons are limited.

In addition to the BAI and GAD-7, the STAI-C, the revised child-specific version of the State-Trait Anxiety Inventory (Spielberger, Gorsuch, \& Lushene, 1970), has been used to gauge symptoms of anxiety. The STAI-C is a 40-item self-reporting measure that assesses both state (20-items) and trait anxiety (20-items). However, although one of the most commonly used measures of anxiety (Julian, 2011) that is specific to children and adolescents (Thabrew, Mcdowell, Given, \& Murrell, 2017), it has not been validated against a gold-standard measure. Moreover, in accord with the lack of validating research, as with the BAI, the STAI-C requires a usage fee, thereby limiting its usability, particularly within studies with small budgets.

Regarding the experimental chapters within the current thesis, the limitations of the BAI and STAI-C, most notably the cost of their reproduction for use within research, make their utilisation unfeasible. Taken together, given the supporting research (Herr et al., 2014; Mossman et al., 2017), coupled with the short nature, it could be suggested that the GAD-7 is the most appropriate tool to measure symptoms of anxiety. 


\section{Measures of depression}

For depression, some of the most popular and commonly utilised tools include the Beck Depression Inventory (BDI; Beck, Ward, Mendelson, Mock, \& Erbaugh, 1961), the Hamilton Rating Scale for Depression (HAM-D; Hamilton, 1960) and the Center for Epidemiologic Studies Depression Scale for Children (CES-DC; Weissman, Orvaschel, \& Padian, 1980). The BDI, considered the gold standard self-rating scale (Cusin, Yang, Yeung, \& Fava, 2010), consists of 21 items and is widely used within adult and adolescent populations (K. Myers \& Winters, 2002). Specifically, the BDI has been shown to be an easy (Thabrew et al., 2017) and reliable (Stockings et al., 2015) measure of depressive symptoms in adolescents. Despite this, the applicability of the BDI to young people has been criticised, with some research suggesting that a lack of items concerning school functioning may limit its use among young people (Kovacs \& Beck, 1977). Copyright restrictions and associated costs may also limit the use of the BDI.

One of the earliest scales to be developed, the HAM-D, consisting of 17-items is the most frequently used depression scale within clinical trials (Cusin et al., 2010). With high and adequate reliability and validity within the general (Simmons, Wilkinson, \& Dubicka, 2015) and adolescent population (Brooks \& Kutcher, 2001), respectively, the HAM-D is quick and easy tool to use (Brooks \& Kutcher, 2001). However, given that the HAM-D is clinician measured, in order to administer this tool a considerable level of training is required (Brooks \& Kutcher, 2001). Furthermore, although the reliability and validity is acceptable, the scale is not youth specific with little data youth data available (K. Myers \& Winters, 2002).

Another scale that has been frequently used within paediatric depression research is the CES-DC, a modified child version of the Center for Epidemiologic Studies Depression Scale (Radloff, 1977). Like the BDI, the CES-DC is a 20-item selfreported scale, but is not copyrighted and, therefore, is freely available to use. Further advantages of the CES-DC for use within research in adolescents is that the measure is sensitive to treatment effects (Simmons et al., 2015) with research suggesting that it is a valid and reliable measure in adolescents (K. Myers \& Winters, 2002). Notwithstanding its advantages, the CES-DC does have its limitations. It has been suggested that the scale measures general psychopathology rather than depression (Brooks \& Kutcher, 2001; K. Myers \& Winters, 2002). Additionally, there have been 
criticisms of its reliability for children, with boys' reliability values particularly poorer than girls' (K. Myers \& Winters, 2002).

Given these findings, in the context of the experimental chapters within this thesis, considering the need for a valid, reliable, inexpensive and self-reported depression assessment tool for young people, the CES-DC is an appropriate scale for use within prospective studies.

\section{Measures of quality of life}

Measurement tools that assess QoL in young people are vast (Drotar, 2004; Eiser \& Jenney, 2007), however, two of the most widely used and validated measures of generic QoL include the KIDSCREEN (Ravens-Sieberer et al., 2005) and the Pediatric Quality of Life Inventory (PedsQL; Varni, Seid, \& Rode, 1999). Promoted by the European Union, the KIDSCREEN is a relatively new multi-version (57-, 27- and 10item) and multi-language self-reporting tool. Although reported by those who developed the measurement tool, the KIDSCREEN has been shown to be a sensitive, valid and reliable measure of QoL (Ravens-Sieberer, Herdman, et al., 2014) and has been recommended in a review of QoL measures (Janssens, Gorter, Ketelaar, Kramer, \& Holtslag, 2008). Although a valid and reliable QoL measurement tool, the 57-item version is relatively time-intensive, with an estimated completion time of 15-20 minutes. As such, the KIDSCREEN may not be a feasible tool in time-pressed environments.

Akin to the KIDSCREEN, the PedsQL is also a self-reported inventory commonly used and widely validated within adolescent populations (Varni, Burwinkle, \& Seid, 2006; Varni, Burwinkle, Seid, \& Skarr, 2003; Varni, Seid, \& Kurtin, 2001). Specifically, the PedsQL is a 23-item self-reporting measure of QoL that has multiple versions for specifically-aged populations. Indeed, the PedsQL has been recommended by a number of reviews as the leading measure of QoL in young people (Eiser \& Morse, 2001; Janssens et al., 2008) and, when compared to other tools, has few, if any, limitations.

Pertaining to the utilisation of a QoL measurement tool within the experimental chapters of this thesis, whilst both the PedsQL and KIDSCREEN are both been equally commended (Janssens et al., 2008), since it has fewer items and has smaller estimated 
completion time, it could be suggested that the PedsQL is the most appropriate tool to measure QoL.

\section{Physical activity}

Physical activity, defined as any bodily movement produced by the skeletal muscles that requires energy, is universally endorsed as a leading preventative measure for a range of physiological health risks (Ekelund et al., 2016; Kyu et al., 2016; J. Myers et al., 2015). Within child and adolescent populations, a number of studies have established a strong inverse relationships between physical activity and physical health, with those that regularly achieve children's physical activity targets set by the Department of Health (2011) at lesser risk of poor physical health. Specifically, children and adolescents that complete 60 minutes of moderate-to-vigorous physical activity per day and undertake vigorous-intensity activities that strengthen muscle and bone three days a week are more likely to obtain significant health benefits (Janssen \& LeBlanc, 2010; Kesäniemi, Riddoch, Reeder, Blair, \& Sørensen, 2010). However, despite research by Martinez-Gomez et al. (2010) suggesting that 60 minutes moderate-to-vigorous physical activity per day adequately prevents increases in adolescents body composition, there have been criticisms and concerns that these guidelines have been established on limited evidence (Donovan et al., 2010). Indeed, current guidelines are based on self-reported physical activity data (Füssenich et al., 2016), which has a number of limitations (Paulhus \& Vazire, 2005). Furthermore, research by L. B. Andersen et al. (2006) exploring the effects of physical activity and cardiovascular risk in children has suggested that 116 minutes of physical activity, approximately double the recommended guidelines, is required to benefit health,.

In addition to criticisms of physical activity guidelines, there has also been a debate regarding the quantity of physical activity. For example, numerous large studies within adult populations have shown that $\sim 150$ minutes of moderate physical activity per week, which is proportionate to 10 metabolic equivalent hours per week, is associated with a lower risk of cardiovascular disease and premature mortality. To accumulate 10 metabolic equivalent hours per week, partaking in only $\sim 75$ minutes of vigorous physical activity (VPA) is required (Manson et al., 2002; Tanasescu et al., 2002). As such, some have argued that researchers should be more concerned with the type of physical activity rather than the volume (A. H. Laursen, Kristiansen, Marott, Schnohr, 
\& Prescott, 2012; Saint-Maurice, Troiano, Berrigan, Kraus, \& Matthews, 2018). Indeed, within child and adolescent populations it has been recommend that vigorous intensity activity should be included when possible as it may provide additional health benefits beyond moderate-intensity physical activity (Janssen \& LeBlanc, 2010). Regardless of these findings, the adage, "some is good; more is better", should still be applied. Indeed, according to a recent systematic review, compared with highly active individuals, those that do not meet the recommended guidelines are $26 \%$ and $21 \%$ more likely to suffer from a stroke or coronary heart disease, respectively (Kyu et al., 2016). Furthermore, as evidenced by Tan et al. (2017), similar outcomes were also noted between physical activity and dementia, the leading cause of death in the UK (Office for National Statistics, 2017).

In addition to physiological or cognitive ailments, increased physical activity has also been shown to significantly enhance mental well-being (Biddle \& Asare, 2011; Fox, 1999; Penedo \& Dahn, 2005). Moreover, considering the financial pressures being faced by the National Health Service (Robertson, Wenzel, Thompson, \& Charles, 2017), physical activity has been recommended as cost-effective approach to rectify such poor health (Hagger, Chatzisarantis, \& Biddle, 2001; Larun, Nordheim, Ekeland, Hagen, \& Heian, 2006).

\section{Effects of physical activity on mental health}

Although the benefits of physical activity on mental health are now widely accepted (Biddle \& Asare, 2011), early reports suggested that its effectiveness was ambiguous, with a report by the Surgeon General stating that:

"The mental health benefits of physical activity for individuals of relatively good physical and mental health were not as evident, but the studies did not have sufficient rigour from which to draw unequivocal conclusions" (US Department of Health and Human Services, 1999).

Furthermore, even more recently it has been reported that there is a limited awareness among clinical psychology professionals as to the effects of physical activity on mental health (Faulkner \& Biddle, 2001). Regardless, however, the effects of increased physical activity are promising (Biddle \& Asare, 2011). Indeed, given the minimal cost, dearth of negative side effects and the adjunct physiological benefits compared 
to alternative pharmacological and non-pharmacological treatments, physical activity is ideally positioned to improve mental well-being (Phillips, Kiernan, \& King, 2003).

\section{Effects of physical activity on anxiety}

Anxiety is an emotion in which a person experiences recurring feelings of tension and intrusive thoughts or concerns (Kowalski, 2000). Studies advocating the association between physical activity and anxiety within the general adult population are vast. Indeed, a recent meta-meta-analysis that identified 306 study effects across 10,755 participants indicated a significant small effect (Rebar et al., 2015). Additionally, although dated, in a large study of US and Canadian participants aged 10 to 74 years, physical activity was shown to be associated with anxiety, irrespective of education and socio-economic status (Stephens, 1988). Despite the evidence suggesting that elevated levels of physical activity can be beneficial for symptoms of anxiety within the general population, there remains a dearth of research focusing specifically on children and adolescents.

Despite the paucity of research, however, there is some evidence to support increased physical activity as a means of improving symptoms of anxiety. Although much of the cohort falls outside of what is considered the upper age limit of an adolescent or child, from a cohort of 2,458, Ströhle et al. (2007) found that those who were regularly active were less likely to suffer from anxiety disorders. Furthermore, those who engaged in regular physical activity had a lower incident rate of anxiety disorders compared to inactive individuals. In addition to issues relating to the relevance of the cohort, these findings are also limited by the lack of objectively measured physical activity. Nonetheless, congruent with Ströhle et al. (2007), a recent study of 481 adolescents (McDowell, MacDonncha, \& Herring, 2017) also supports physical activity as a means to improve anxiety. Indeed, it was reported that moderate and high physical activity were inversely related to anxiety. Again, however, the validity of the recorded physical activity is questionable given that a modified version of the self-reporting PatientCentred Assessment and Counselling for Exercise Plus Nutrition (Prochaska, Sallis, \& Long, 2001) was used to measure physical activity.

Consistent with McDowell et al. (2017) and Ströhle et al. (2007), a study examining the association between self-reported physical activity and anxiety in 1,747 Malay adolescents also found that the level of physical activity was significantly associated 
with symptoms of anxiety (Tajik et al., 2017). Though, it should be noted that in addition to using a self-reported physical activity, anxiety was measured using the 21item Depression, Anxiety and Stress Scale (Lovibond \& Lovibond, 1995), which has provided mixed findings regarding its validity for use within adolescents (Le et al., 2017; Patrick, Dyck, \& Bramston, 2010).

Contrary to McDowell et al. (2017), Ströhle et al. (2007) and Tajik et al. (2017), earlier research (Norris, Carroll, \& Cochrane, 1992; Strauss, Rodzilsky, Burack, \& Colin, 2001) has suggested that physical activity levels are not related to symptoms of anxiety. Norris and colleagues (1992), who collected self-reports of exercise and psychological stress and well-being from 147 adolescents, suggested that anxiety, measured using the Multiple Affect Adjective Check List (MAACL; Zuckerman \& Lubin, 1990), shared no significant correlation with physical activity, or exercise as they have described it. However, whilst physical activity measured using self-reported methods is already questionable, these results were further limited given that the selected physical activity questionnaire (J. D. Brown \& Lawton, 1986) failed to account for habitual physical activity outside of exercise.

Regardless of methodological shortcomings relating to self-reported measures (Norris et al., 1992), using objectively measured physical activity, Strauss et al. (2001) also found that, within a cohort of 95 10-16 year olds, anxiety, irrespective of if it was measured using the Revised Children's Manifest Anxiety Scale (C. R. Reynolds \& Richmond, 1985) or the Piers-Harris inventory (Piers \& Harris, 1969), was related to physical activity. Although rigorous in terms of its physical activity measurement, this study is, however, limited by the relatively small sample size when compared to other studies (McDowell et al., 2017; Norris et al., 1992; Ströhle et al., 2007; Tajik et al., 2017), and is perhaps underpowered.

In summary, whilst the interpretation of each of the studies within this section is limited, given the considerably greater total sample size when combining McDowell et al. (2017), Ströhle et al. (2007) and Tajik et al. (2017), compared to Norris et al. (1992) and Strauss et al. (2001), it could be postulated that physical activity can ameliorate anxiety in adolescents. Nonetheless, future research is needed to provide greater clarification as to physical activity's effects on anxiety in children. 


\section{Effects of physical activity on depression}

Depression is a mental illness whereby a person experiences a lack of interest and pleasure in daily activities, experiences weight loss or gain, and/or suffers from insomnia or excessive sleeping. Additional symptoms include feeling lethargic, an inability to concentrate, experiencing feelings of worthlessness, excessive guilt or recurrent thoughts of death or suicide (Beckham, 2000). Within adults, research has shown that higher levels of physical activity are often indicative of improved mental health (Rebar et al., 2015). The relationship between physical activity and mental health has also been established within children and adolescents (Kremer et al., 2014; McMahon et al., 2017).

Following a large-scale study ( $\mathrm{n}=8,256,10-16$ years) that examined the associations between self-reported physical activity levels and depressive symptoms, measured via the Short Mood and Feelings Questionnaire (Angold et al., 1995), Kremer and associates (2014) reported that those who participated in physical activity were less likely to suffer from depressive symptoms. Furthermore, those participants that were more frequently physically active at school, both within and outside of class, and/or met moderate-to-vigorous physical activity guidelines, reported fewer symptoms of depression. These findings therefore suggest that future research should heavily consider school-based physical activity interventions if aiming to ameliorate symptoms of depression in adolescents. However, it should be noted that the differences between the low and moderately active participants was small and may not have been clinically significant.

In a more recent large-scale $(\mathrm{n}=11,110)$ cross-sectional study in adolescents (mean age $=14.8$ years) across Europe, findings suggested that the frequency of self-reported physical activity was also correlated with depression (McMahon et al., 2017). McMahon and colleagues (2017) found that depressive symptoms, measured using the Beck Depression Inventory II (Beck, Steer, Ball, \& Ranieri, 1996), were negatively correlated with the frequency of physical activity. Interestingly, whilst lower levels of physical activity were associated with depression, an upper threshold was also detected. Indeed, among girls, exceeding 60 minutes of physical activity on more than 11 days within a 2-week period was associated with higher levels of depression. Consequently, it could be postulated that these findings support the use of short intense exercise, such as HIIT, as a means of improving health given the lesser total exercise 
time and associated benefits (Costigan, Eather, Plotnikoff, Taaffe, \& Lubans, 2015; Logan et al., 2014). However, this is perhaps speculative given that physical activity was self-reported with no information as to the intensity of that exercise. Also, it is possible that the upper threshold may well be associated with energy expenditure, which is comparative when considering the exercise intensity and time ratio. Furthermore, as suggested within McMahon et al. (2017), this may be a result of the sub-group that reported a high frequency of physical activity over-exercising and/or suffering from eating disorders or additional mental health disorders.

Partially in support of McMahon et al. (2017), research has shown that within children, for each additional hour of physical activity undertaken per week, the odds of experiencing depressive symptoms are decreased by 8\% (Rothon et al., 2010). However, despite these positive findings (Kremer et al., 2014; McMahon et al., 2017; Rothon et al., 2010), it should be noted that all of the studies measured physical activity using self-report methods, thus bringing into question the validity and subsequent underestimation of physical activity levels and overall findings.

In contrast to Kremer et al. (2014) and McMahon et al. (2017), within a large sample of adolescent girls $(n=1,397)$, Johnson et al. (2008) did not observe any significant relationship between physical activity and self-reported depression. These findings are especially compelling given that physical activity was measured objectively via accelerometery. Furthermore, although the depression measure, the Center for Epidemiological Studies Depression Scale (Radloff, 1977), is not child-specific, a later study (Radloff, 1991) validated its use in children. However, unfortunately this study only included girls and, therefore, may not be representative of the wider population. Furthermore, the data within this study was from a wider school-based intervention aimed at increasing physical activity (Stevens et al., 2005), therefore, the effects of self-selection bias (i.e., only those that were fit and healthy volunteered to take part) may also limit these findings.

Whilst Johnson et al. (2008) were perhaps more rigorous in terms of measurement validity, when comparing the level of evidence in terms of sample size, Kremer et al. (2014) and McMahon et al. (2017) provided a strong argument that increased physical activity can improve symptoms of depression. As such, although it could be tentatively 
summarised that physical activity levels are indicative of depressive symptoms in children and adolescents, more research is required substantiate this.

Given the relationship between physical activity and anxiety and depression in children and adolescents (Kremer et al., 2014; McDowell et al., 2017; McMahon et al., 2017; Ströhle et al., 2007; Tajik et al., 2017), it is possible that the poor state of child and adolescent mental health (Collishaw et al., 2010; Green et al., 2004) is symptomatic of current physical activity levels. Indeed, reports suggest that in England less than $23 \%$ of children and adolescents meet UK physical activity guidelines (Ryley, 2013; Scholes, 2016). This is especially worrying considering that physical activity during a person's formative years has been shown to influence physical activity habits and health in later life (Blair, Clark, Cureton, \& Powell, 1989; Dumith et al., 2011). Consequently, although few effective mental health prevention strategies have been established (McCrone et al., 2008), exercise, the structured form of physical activity, has been proposed as a means that can increase physical activity levels and subsequently improve health and well-being.

\section{Exercise}

Exercise, a sub-component of physical activity that is carried out to sustain or improve health, has frequently been utilised to improve physical health (Anderson et al., 2016; Farina, Rusted, \& Tabet, 2014; Haskell, Lee, Pate, Powell, \& Blair, 2007; Janssen \& LeBlanc, 2010). Within children and adolescents, research has shown that prescribing exercise, such as resistance or interval training, can significantly improve physical activity levels and mitigate the risk of health issues such as type 2 diabetes mellitus, cardiovascular disease and obesity (Dias et al., 2017; Shiroma et al., 2017; K. L. Weston et al., 2016). However, whilst there has been a considerable interest in physical health, mental health and QoL have garnered significantly less attention within child and adolescent populations. Indeed, the psychological effects of fitness training were originally viewed by some physiologists and psychiatrists as having been "propagandized by the popular press" (Folkins \& Sime, 1981). However, more recently, exercise has been supported as a treatment for mental health issues by a number of national bodies (Gelenberg et al., 2010; National Collaborating Centre for Mental Health and The National Institute for Health and Care Excellence, 2010). 


\section{Effects of exercise on mental health}

The beneficial effect of exercise on mental health and well-being is evident across a wealth of populations. Exercise has been demonstrated to improve psychological health and well-being in older adults (Netz, Wu, Becker, \& Tenenbaum, 2005), those suffering from chronic illness or disability (Graham, Kremer, \& Wheeler, 2008), pregnant women (Da Costa, Rippen, Dritsa, \& Ring, 2003) and the general population (Hassmén, Koivula, \& Uutela, 2000). However, despite research suggesting that physical activity can positively influence mental well-being (Kremer et al., 2014; McMahon et al., 2017; Rothon et al., 2010), the effect of exercise on psychological health and well-being in children and adolescents has accumulated less attention (Whitelaw, Teuton, Swift, \& Scobie, 2010). However, regardless of the paucity of research, whilst dated, several studies have explored the effect of physical activity and exercise on anxiety and depression in child and adolescent populations.

\section{Effects of exercise on anxiety}

Research within child and adolescent populations is sparse, however, evidence suggests that exercise may elicit a reduction in anxiety. Early research using youth offenders ( $n=60,15-18$ years) concluded that, in addition to physiological measures, self-reported anxiety, measured via the State-Trait Anxiety Inventory for Children (Spielberger, 1973), improved as a result of a 20-week intervention consisting of three 90-minute exercise sessions per week (Hilyer et al., 1982). Hilyer et al. (1982) noted that whilst the intervention group $(n=30)$ scored less favourably than the control group $(n=30)$ at baseline, post-intervention scores were reversed, highlighting the magnitude of the improvement. However, the generalisation of these findings to the wider population may not be appropriate given the circumstances of the participants.

Similar to Hilyer et al. (1982), Norris, Carroll and Cochrane (1992) who, when analysing participants from the general adolescent population $(n=80)$, found that after a 10-week intervention, anxiety, measured using the MAACL (Zuckerman \& Lubin, 1990), improved in the exercise groups. Furthermore, while significant improvements in anxiety were manifest in both exercise intensity groups, Norris and colleagues (1992) suggest that the high-intensity group elicited the greatest improvement. Caution should, however, be taken when interpreting these findings given that the specified exercise intensity does not meet the standards considered to be high-intensity by recent 
research (Hood, Little, Tarnopolsky, Myslik, \& Gibala, 2011; Little et al., 2011). In support of Hilyer et al. (1982) and Norris et al. (1992), a more recent and longitudinal study, that included 197 adolescents, suggested that anxiety, measured using the Hospital Anxiety and Depression Scale (HADS; Bjelland, Dahl, Haug, \& Neckelmann, 2002), significantly improved relative to the control $(-2.8 \%, p<0.01$; Bonhauser et al. 2005). However, given that Bonhauser et al. (2005) focused on participants of lower socioeconomic status in Chile, these findings may not represent the general global adolescent population.

In contrast to the previous positive findings (Bonhauser et al., 2005; Hilyer et al., 1982; Norris et al., 1992), despite eliciting significant physiological improvements, Lau, Yu, Lee and Sung (2004) found no significant differences in anxiety measured using the HADS (Bjelland et al., 2002). In this study 36 obese adolescents (24 boys) aged 10-17 years were recruited and assigned to either an intervention or control group. For the intervention group, participants were required to complete 3 resistance training sessions per week for 6 weeks in conjunction with a hypocaloric diet, whilst the control group followed dietary intervention only. Such mixed findings may, in part, be due to the poor quality of the study (Larun et al., 2006). Lau et al. (2004) acknowledged that their sample size was not adequate and, therefore, may not have been suitably powered to detect significant differences. Furthermore, it could be postulated that the lack of significant findings may have been attributed to the relatively healthy state of anxiety in both the intervention and control groups which, consequently, negated the potential for anxiety to improve as a result of exercise.

In summary, possibly due to publication bias, to the authors knowledge, there are no other studies in children or adolescents that have reported no significant changes. Therefore, in summary, given the minimal evidence in opposition, it is possible to suggest that exercise an effective means of reducing symptoms of anxiety, however, further research is required.

\section{Effects of exercise on depression}

Although sensations of depression have increased two-fold within adolescents (Collishaw et al., 2010), research exploring the effects of exercise on depression is sparse (Biddle \& Asare, 2011). As with anxiety, Hilyer et al. (1982) conducted one of the earliest studies in children and adolescents. Following 20 weeks of flexibility, 
resistance or aerobic training 3 times per week, Hilyer and associates (1982) reported that symptoms of depression significantly improved between pre- and post-test in the intervention group, despite a significant increase in the control group. Congruent with Hilyer et al. (1982), a more recent study in the general population found similar results (Hughes et al., 2013). In Hughes et al. (2013), a small sample of adolescents ( $n=30$, 12-18 years) completed either a 12-week thrice weekly exercise or stretching intervention. Although symptoms of depression, measured using the Children's Depression Rating Scale-Revised (Poznanski \& Mokros, 1996), significantly decreased in both groups and the depressions scores between the exercise group and stretching control group not being significantly different at post-intervention, these results still advocate exercise as a means to improve exercise since both interventions groups induced some form of energy expenditure. Furthermore, although similar benefits were manifest in both groups, for the exercise group, such improvements were evident at a greater magnitude.

Significant changes to depression were also reported by Jeong et al. (2005). In this study, matched by an equal number of control participants, 20 adolescents suffering from mild depression completed 3 x 45-minute dance therapy sessions over 12 weeks. Results suggested that depression scores, measured via the Symptom Check List-90Revision (Derogatis, 1992), significantly deteriorated in the control group, while mean depression scores significantly improved in the exercise group. However, these findings are limited given the small sample size and the absence of an equivalent exercise control group to estimate an expectation effect. Despite these positive outcomes (Hilyer et al., 1982; Hughes et al., 2013; Jeong et al., 2005), it should be noted that, although commonly utilised due to their time- and cost-effectiveness, all psychological outcome measures were self-reported. As such, the validity of these findings should be questioned given the inherent issues, such as social desirability (Paulhus \& Vazire, 2005), associated with self-report measures.

Converse to previous studies (Hilyer et al., 1982; Hughes et al., 2013; Jeong et al., 2005), some research has suggested that exercise has no effect on depression in children or adolescents (Bonhauser et al., 2005; S. W. Brown, Welsh, Labbé, Vitulli, \& Kulkarni, 1992). Despite reporting significant reductions in anxiety, Bonhauser et al. (2005) suggest that exercise does not elicit any beneficial effects to symptoms of depression measured using the HADS (Bjelland et al., 2002). However, in addition to 
the previously stated limitations, given that physical activity was not tracked throughout this study, such findings may, in part, be due to a compensation effect whereby increased physical activity during one part of the day may result in a compensatory decrease during another part (Ridgers, Timperio, Cerin, \& Salmon, 2014). Whilst limited, the findings from Bonhauser and colleagues (2005) are supported by additional literature (S. W. Brown et al., 1992). In S. W. Brown et al. (1992), following 3 exercise sessions per week over 9 weeks, when baseline was compared to post-intervention and follow-up scores, no significant differences to depression, measured using the BDI (Beck et al., 1961), were observed. The quality of these findings should, however, be questioned given the numerous limitations of the study. Due to poor adherence, from 27, the final participant cohort of 11 participants did not provide adequate statistical power to detect significant changes. Furthermore, whilst not diagnosed with depression, all the participants included within this study were inpatients at a psychiatric institution. As such, their underlying mental health issues, primarily dysthymia and conduct disorder, could have had some bearing on the outcome of this study and, subsequently, restrict the application of these findings within the general population.

Given these findings, taking in the large body of evidence (Hilyer et al., 1982; Hughes et al., 2013; Jeong et al., 2005), it could be postulated that exercise is an effective means of improving symptoms of depression in children and adolescents. However, given the various limitations of studies included within this section, the effect of exercise on depression is still ambiguous, and therefore more research is needed.

\section{Dose-response of physical activity and exercise interventions on mental health}

Physical activity and exercise dose-response typically refers to three discrete variables; duration, frequency and intensity. Duration concerns the amount of time accrued in a single exercise session or an intervention as a whole, with frequency reflecting the number of exercise sessions over a specified period, for example a week. Whilst duration and frequency are basic concepts that concern the total time spent in exercise, intensity is perhaps slightly more convoluted. Intensity is "typically quantified in absolute terms as the metabolic cost of an exercise session or in relative terms as the performance of a given activity as a function of some percentage of measurable maximal capacity" (Wasfy \& Baggish, 2016, p. 2298). In addition to duration, 
frequency and intensity, another variable that is commonly included is the modality, or type of exercise, for example, the activity within an exercise intervention (i.e. running, resistance training or swimming).

\section{Anxiety}

With the exception of a limited number of studies that have explored the effects of intensity (Norris et al., 1992; Parfitt et al., 2009), research exploring the dose-response of physical activity and exercise interventions with regard to duration and frequency on anxiety in children and/or adolescents is limited. From a cohort of 82 children (32 boys) aged between 9-10 years, utilising objectively measured physical activity and symptoms of anxiety scored using the STAI-C (Spielberger \& Edwards, 1973), Parfitt et al. (2009) suggested that different intensities of physical activity were associated with children's psychological health. Indeed, those children that accumulated high levels of lighter-intensity physical activity reported greater symptoms of anxiety in comparison to those who accrued more VPA. Furthermore, these results remained when body fatness, a factor that has been positively associated with anxiety within this population (Esposito et al., 2014), was controlled for. Similarly, Norris et al. (1992) found that exercise intensity was associated with adolescent anxiety. Within this study, 44 participants with a mean age of 16.7 years were allocated to either a 10 -week highintensity $(n=14)$, moderate-intensity $(n=15)$ or flexibility $(n=15)$ school-based intervention. The exercise sessions for each protocol lasted between 25-30 minutes and were completed twice per week. It was reported that, compared to the moderateintensity group, those in the high-intensity group experienced significant reductions to anxiety post-intervention. However, although both these studies (Norris et al., 1992; Parfitt et al., 2009) advocate VPA and high-intensity exercise, the results should be interpreted with caution due to their small sample sizes. Additionally, within Norris et al. (1992), whilst referred to as high-intensity, the information relating to participant HR is limited. As such, it is difficult to ascertain whether the exercise intensity met modern high-intensity criteria (Hood et al., 2011; Little et al., 2011).

Despite a paucity of information within children and adolescents, in adults there has been considerably more attention as to the influence of dose-response of physical activity and/or exercise on anxiety. Whilst studies are yet to examine the combined effect of intensity, duration and frequency of exercise or physical activity on anxiety, 
some information is available via numerous studies that have analysed components individually. With reference to the influence of intervention duration, in a metaanalysis, Petruzzello, Landers, Hatfield, Kubitz and Salazar (1991) concluded that the length of the intervention was significantly associated with effectiveness. Their findings suggested that those who trained for 16 weeks or longer benefited from greater improvements to anxiety than those who trained for 9 weeks or less. Petruzzello and colleagues (1991) also suggested that, for improvements to anxiety to be manifest, a minimum of 21 minutes of exercise is required. The interpretation of these findings, however, are restricted by confounding factors related to the design or exercise variables within the included studies.

Akin to exercise duration, the frequency of physical activity or exercise sessions was also shown to influence symptoms of anxiety. Indeed, as suggested in their metaanalysis, Wipfli, Rethorst and Landers (2008) concluded that participating in exercise 3-4 times per week elicits greater benefits to anxiety than exercising 1-2 times a week. Interestingly, Wipfli et al. (2008) also indicated that there may be a maximum threshold to exercise frequency, in that those that engage in exercise more than 4 times a week yield fewer benefits than those who exercise 3-4 times per week.

In contrast to the findings within children and adolescents, an optimal intensity within the adult population was more ambiguous. Specifically, although there are no studies that have specifically examined differing intensities, there is support for both aerobic and anaerobic exercise as means to improve anxiety. Furthermore, the effect of differing intensities of long-term or chronic physical activity or exercise are comparable. Indeed, aerobic training consisting of moderate-intensity exercise (Blumenthal, Williams, Needels, \& Wallace, 1982; DiLorenzo et al., 1999) and anaerobic training consisting of high-intensity resistance training (Cassilhas, Antunes, Tufik, \& de Mello, 2010; Tsutsumi, Don, Zaichkowsky, \& Delizonna, 1997) have both been found to elicit similar significant benefits to anxiety.

With reference to the most beneficial exercise type or modality, whilst direct comparisons are not available due to limited research, according to a recent systematic review (Stonerock, Hoffman, Smith, \& Blumenthal, 2015), aerobic type exercises such as running are the most common form of exercise to treat anxiety. However, as alluded to previously, the effects of both aerobic and resistant type exercises on anxiety are 
comparable (Cassilhas et al., 2010; Tsutsumi et al., 1997). Whilst these findings provide a useful insight into the dose-response of physical activity and exercise on anxiety, it should be noted that their findings are limited in the context of paediatric research given that children are not mini-adults. As such, further research is required exploring the effects of different doses of physical activity and/or exercise on anxiety in children and adolescents.

\section{Depression}

In comparison to anxiety, with the exception of physical activity and/or exercise modality, for which there is a paucity of information, there is a relative wealth of research exploring the dose-response of physical activity and/or exercise and its effects on depression in children and adolescents. Using a large adolescent sample $(n=1,396)$, based on the WHO Composite International Diagnostic Interview (CIDI, R. C. Kessler \& Üstün, 2004) depression scores and subjectively measured physical activity, Stavrakakis et al. (2013) suggested that a dose-response does not exist. Specifically, Stavrakakis and colleagues (2013) reported that the nature, frequency, duration and intensity of physical activity were not significantly indicative of depression. Despite these findings (Stavrakakis et al., 2013), several methodological issues limit such an interpretation. Indeed, physical activity was measured using self-report, which can overestimate the amount of time engaged in, and the intensity of physical activity (Hussey, Bell, \& Gormley, 2007). Furthermore, although the CIDI uses an interview to evaluate a person's level of depression, its format replicates a questionnaire that can be administered by a non-health professional and, therefore, may not be any more valid or reliable than a self-reporting questionnaire. Regardless of these limitations, Norris et al. (1992) also concluded that exercise intensity had no effect on depressive symptoms. In this randomised control trial, participants were allocated to either a 30minute bi-weekly 10-week high-intensity $(n=14)$, moderate-intensity $(n=15)$ or flexibility $(n=15)$ intervention. Results suggested that none of the intensities had any effect on depression, which also suggests that exercise in general does not elicit improvements to depression in adolescents. However, the interpretation of these findings are perhaps limited given the small sample size of the overall study, and especially when analysed in their separate groups. Additionally, insufficient information relating to participants' HR during exercise makes it difficult to ascertain the true exercise intensity. 
Whilst both Norris et al. (1992) and Stavrakakis et al. (2013) found that the intensity of physical activity and exercise had no effect on depression, Goldfield et al. (2011) and Tao et al. (2007) found that differing intensities of physical activity did elicit contrasting effects on depression. In a study of 1,259 adolescents (Goldfield et al., 2011), physical activity and depression, measured via self-reporting methods and the Child Depression Inventory (Kovacs, 1992), respectively, were correlated. A clear dose-response was reported in relation to physical activity intensity, whereby VPA exhibited greater effects to depressive symptoms compared to mild- and moderateintensity physical activity. Although, when dichotomised by sex, VPA was only significantly associated with depression in boys. Alternatively, as part of a large-scale study ( $\mathrm{n}=5,453)$, Tao et al. (2007) reported that whilst light-to-moderate intensity physical activity protected against the development of depressive symptoms, measured using the Symptoms Checklist 90 (Z. Y. Wang, 1999), high-intensity physical activity was shown to significantly increase the risk of general mental health problems in school children. However, the difference in findings compared to Goldfield and colleagues (2011) may be explained by cultural discrepancies, in that Tao et al. (2007) focused exclusively on Chinese adolescents. In addition to the disparity between studies (Goldfield et al., 2011; Tao et al., 2007), physical activity was again reported using self-reported measures, casting doubt over the validity of the true intensities of physical activity.

In relation to physical activity duration, in a sample of 2,093 participants aged between 11 and 14, Rothon et al. (2010) found that depression, measured using the Short Moods and Feelings Questionnaire (Angold et al., 1995), was associated with the duration of physical activity. Interestingly, Rothon et al. (2010) also reported that for each additional hour of physical activity or exercise recorded per week, depressive symptoms reduced by $8 \%$. These findings therefore suggest that a greater duration of physical activity is more beneficial. Though, it should be stated that physical activity was measured using self-report methods that did not take in to account physical activity within school, during physical education classes or at school break times, and as such is likely unreliable. In terms of exercise intervention duration, in a metaanalysis which evaluated 9 studies encompassing a sample size of 581 children and adolescents (H. E. Brown, Pearson, Braithwaite, Brown, \& Biddle, 2013), it was 
suggested that shorter duration interventions were more effective. Indeed, the largest effects that were noted were for interventions lasting less than 3 months.

Within H. E. Brown et al. (2013) the frequency of exercise sessions and their effect on depression was also assessed, however, there was no significant difference between less than three, or more than four, sessions per week. In contrast to H. E. Brown et al. (2013), McMahon et al. (2017) suggested that physical activity frequency can effect symptoms of depression. From a sample of 6,566 adolescents, McMahon et al. (2017) found that exceeding 60 minutes of physical activity on more than 11 days within a 2week period was associated with higher levels of depression. These findings indicate the existence of an upper threshold, and as such, it could be suggested that achieving the governmental guidelines of 60 minutes moderate-to-vigorous physical activity may be detrimental to depressive symptoms. However, these findings may be a result of over-exercising and/or participants suffering from eating disorders or additional mental health disorders.

\section{Further considerations of a dose-response in relation to physical activity and exercise}

Whilst it could be postulated that moderate- and high-intensity exercise are perhaps equally as effective in improving anxiety and depression, it has been suggested that there are distinct differences between exercise intensity and affective responses (Ekkekakis, 2003). Referred to as the dual-mode theory (Ekkekakis, 2003), this theoretical framework proposes that affective responses to exercise are jointly influenced by cognitive factors and interoceptive cues, the balance between which fluctuate in relation to exercise intensity. For example, during and for a limited period after moderate-intensity exercise, the majority of people respond positively. This theory further posits that, during exercise performed at or near ventilatory threshold, individuals' response can vary significantly, with some reacting negatively and some positively. This framework further postulates that when exercise is performed beyond the ventilatory threshold, there is a significant decline in the valence of affective responses. Essentially, Ekkekakis (2003) asserts that as exercise intensity increases, pleasure decreases. Whilst such findings should be considered with regards to the effects of dose-response, it should be noted that the majority of the research on the dual-mode model has involved healthy, relatively active adults, with some of the 
relationships yet to be tested within people of varying ages (D. M. Williams, 2008). Consequently, such findings may not be applicable to the to the paediatric population within this thesis.

\section{Mechanistic basis of physical activity and exercise on mental health}

The beneficial effects of physical activity and exercise on psychological health and well-being in children and adolescents are evident (Bonhauser et al., 2005; Hilyer et al., 1982; Hughes et al., 2013; Jeong et al., 2005; Norris et al., 1992) and several mechanisms have subsequently been proposed that may facilitate such improvements. One physiological adaptation of physical activity and exercise, widely considered to be a primary mechanism of improved mental health is increased cerebral blood flow, which can subsequently affect the hypothalamic-pituitary-adrenal axis. Indeed, physical activity and exercise have been shown to alter the concentration of numerous biologically-active molecules, such as adrenocorticotrophic hormones, cortisol, catecholamines, opioid peptides and cytokines, which have been reported to effect mood (McArdle, Katch, \& Katch, 1996). Further to increased cerebral blood flow, it has been hypothesised that participation in physical activity and/or exercise can serve as a distraction from negative thoughts (Craft, 2005; Craft \& Perna, 2004; Leith, 1994) and improve a person's mood during and after exercise. During exercise, and especially within a competitive environment, a person's thoughts are focused on specific goals related to the chosen activity, reducing the time spent reflecting on negative thoughts. It has also been postulated that exercise can increase self-efficacy, via its ability to provide effective mastery experience (Bandura, 1997), which has been associated with improvements in depressive symptoms (Craft, 2005). The benefit of social interactions during physical activity or exercise, especially the social relationships formed within team sports, have also been shown to improve mental health (Peluso \& de Andrade, 2005; Ransford, 1982). Finally, physiological improvements attributed to physical activity and exercise, such as an improved body composition (Drenowatz et al., 2016; Gutin et al., 2002), have been shown to induce improvements to a person's self-image and self-esteem (C. E. Ross, 1994), both of which have been associated with mental health (Lubans et al., 2016; Markowitz, Friedman, \& Arent, 2008). Whilst the benefits of exercise and physical activity on mental health is evident (Bonhauser et al., 2005; Hilyer et al., 1982; Hughes et al., 2013; Jeong et al., 2005; Norris et al., 1992), most exercise interventions have 
traditionally focused on high-volume, moderate-intensity exercises, which are often time-consuming, a frequently cited barrier to exercise (Donahue et al., 2006; Lovell et al., 2010; Reichert et al., 2007; Welch et al., 2009). Subsequently, in order to ameliorate such issues, HIIT has been identified as a potentially potent and timeefficient form of exercise (Gibala, 2007).

\section{High-intensity interval training}

The beneficial effects of moderate or endurance exercise to both physical and mental health are have been widely evidenced (Bonhauser et al., 2005; Cornelissen \& Fagard, 2005; Donges, Duffield, \& Drinkwater, 2010; Hughes et al., 2013; Sigal et al., 2014), however, such exercise may not be congruous with the time-restricted society of today (Palmer, 2010). Specifically, perceived lack of time is commonly indicted as a barrier to physical activity (Dwyer et al., 2006; Lovell et al., 2010; Reichert et al., 2007; Welch et al., 2009). Furthermore, the relevance of such exercise to the sporadic high-intensity nature of children's habitual play patterns has been questioned (Bailey et al., 1995), and may not be appealing to adolescent populations (Buchan et al., 2011). In an attempt to ameliorate such barriers, research has proposed that individuals may be able to achieve the benefits accrued from meeting governmental physical activity guidelines more time-efficiently by substituting moderate-intensity physical activity with VPA (I.-M. Lee \& Paffenbarger, 2000; Yu, Yarnell, Sweetnam, \& Murray, 2003). Moreover, it has also been suggested that more intense physical activity may elicit greater benefits given the subsequent increased energy expenditure accrued compared to moderate-intensity physical activity of a similar duration (Billat, 2001; Christensen, Hedman, \& Saltin, 1960).

Given the health and time benefits of increased VPA, the implementation of HIIT exercise, a structured form of VPA, has been proposed. Significantly associated with elevated VPA levels (Costigan et al., 2017), there has recently been an upsurge in interest in the potential health-related benefits of HIIT as a time-effective method of exercise (Gibala, Little, Macdonald, \& Hawley, 2012; H. S. Kessler, Sisson, \& Short, 2012; Warburton et al., 2005; M. Weston, Taylor, Batterham, \& Hopkins, 2014). Extensively used by athletes and coaches to improve cardiorespiratory and metabolic function with the aim of maximising performance within elite sport (FernandezFernandez, Zimek, Wiewelhove, \& Ferrauti, 2012; Gibala \& McGee, 2008; P. B. Laursen, Shing, Peake, Coombes, \& Jenkins, 2002; Westgarth-Taylor et al., 1997; A. 
R. Weston et al., 1997; Wisløff, Ellingsen, \& Kemi, 2009), HIIT, also known as highintensity intermittent exercise, can be defined as short and intense repeated bouts of exercise (Buchheit \& Laursen, 2013; P. B. Laursen \& Jenkins, 2002) at "maximum effort" (Buchheit \& Laursen, 2013). Exercise durations range from a few seconds to several minutes (Gibala \& McGee, 2008), interspersed with passive or active recovery intervals (Buchheit \& Laursen, 2013), which ordinarily last a duration equal to that of the exercise bout (L. M. Ross, Porter, \& Durstine, 2016). Exercise interval intensity can be measured using a variety of methods: $\mathrm{HR}$, oxygen uptake $\left(\dot{V} \mathrm{O}_{2}\right)$, rating of perceived exertion and maximal aerobic speed (MAS). High-intensity exercise is usually greater than or equal to $90 \% \dot{V} \mathrm{O}_{2 \text { peak }}$ (Gibala \& McGee, 2008), an intensity equal to or greater than $100 \%$ MAS (Baker, 2011) and/or an exercise induced HR equal to or greater than $90 \%$ maximum HR (Hood et al., 2011; Little et al., 2011).

Whilst the primary appeal of HIIT was that it could be completed in a shorter period of time compared to traditional aerobic training for equal effect, recent systematic reviews and meta-analyses have suggested that HIIT may also stimulate more beneficial changes to health (Costigan, Eather, Plotnikoff, Taaffe, \& Lubans, 2015; Logan et al., 2014). In a recent meta-analysis (Costigan, Eather, Plotnikoff, Taaffe, \& Lubans, 2015), data revealed that HIIT significantly improved cardiorespiratory fitness, BMI and body fat percentage when compared to moderate-intensity training. Similarly, Logan et al. (2014) reported that cardiovascular disease biomarkers and cardiorespiratory fitness were improved to a greater extent with HIIT than moderateintensity exercise. However, despite the widespread use within elite sport and the growing interest of HIIT within public health, the use of HIIT in non-athlete populations was initially considered to be unsafe (MacDonald \& Currie, 2009). Concerns have been raised regarding the feasibility of implementing HIIT in sedentary, overweight or clinical populations, with perceived barriers including risk of injury (Donahue et al., 2006) and participant ability to participate in such highintensity exercise (Korkiakangas, Alahuhta, \& Laitinen, 2009). Specifically, the cardiac strain induced by the high HR naturally elicited by HIIT. However, growing evidence has alleviated these concerns, with recent reviews demonstrating the beneficial effects and safety of HIIT in clinical and general populations (Guiraud et al., 2012; Hwang, Wu, \& Chou, 2011; H. S. Kessler et al., 2012; L. M. Ross et al., 2016; K. S. Weston, Wisløff, \& Coombes, 2014). For example, research has suggested 
that the stop-start exercise pattern allows less fit individuals a greater capacity to perform vigorous exercise given that there are rest periods naturally built in (Francois \& Little, 2015). It is also poignant to note that within the exercise phase of HIIT, the intensity at which an individual exercises is relative to their own cardiorespiratory fitness and is not standardised. Furthermore, a meta-analysis by K. S. Weston et al. (2014) reported that clinical populations suffering from cardiometabolic disease performing HIIT exhibited a greater increase in cardiorespiratory fitness when compared to a moderate-intensity exercise group.

\section{Effects of high-intensity interval training on mental health}

Moderate physical activity is commonly cited as the recommended mode of physical activity in order to elicit improvements in health (Pate et al., 1995). However, work in children and adolescents by Parfitt et al. (2009) found that, although low and moderate physical activity were correlated with negative psychological profiles, VPA was associated with a positive psychological profile. More specifically, Gerber et al. (2014) found that, in adults, VPA was not only associated with less stress, but also that those that met the recommended VPA targets, as set by the American College of Sports Medicine (Haskell et al., 2007), and similar to UK guidelines (Department of Health, 2011), reported fewer mental health issues when exposed to high-stress situations. Furthermore, those that reached VPA targets achieved mental health benefits beyond those experienced with just moderate physical activity. One method of exercise that was identified to increase VPA was HIIT. Although limited, early research found that HIIT did not significantly increase physical activity (Boddy et al., 2010), however, more recent rigorous research has shown that, despite the prospect of the "activitystat" hypothesis (Gomersall, Rowlands, English, Maher, \& Olds, 2013), not only was HIIT able to significantly increase moderate-to-vigorous physical activity (Lonsdale et al., 2013), but also moderately increased VPA levels (Costigan et al., 2017). However, in spite of evidence supporting HIIT as an efficient method to increase VPA, which is as an effective means of improving mental well-being (Gerber et al., 2014; Parfitt et al., 2009), there remains a paucity of research investigating HIIT within a healthy population; with the majority of research exploring the effects HIIT on mental health has been in those suffering from mental illness, such as schizophrenia (Wu, Lee, Hsu, Chang, \& Chen, 2015) or depression (Singh et al., 2005), or those diagnosed with a cognitive impairment, such as dementia (Telenius, Engedal, \& Bergland, 2015). 
Despite the apparent translation of HIIT into VPA and the scarcity of research available, to date, only two studies have examined the effect of HIIT on mental health in healthy adults (Shepherd et al., 2015) and adolescents (Costigan et al., 2016).

In Shepherd et al. (2015), ninety healthy but inactive adults (18-60 years) were randomly assigned to either a HIIT or moderate-intensity continuous training group. Exercise sessions were instructor-led in a gym and were conducted in groups of 10-15 using mechanically-braked spinning bikes. For the HIIT group, participants were asked to attend three 18 to 20 -minute sessions per week for 10 weeks. Within these sessions, in addition to a 5-minute warm-up and cool-down, participants performed multiple repeated high-intensity sprints (>90\% HR maximum) lasting between 15 and 60 seconds, interspersed with active rest lasting 45 to 120 seconds. For the moderateintensity continuous training group, each session also began and ended with a warmup and cool-down. Over 10 weeks participants were asked to attend 3 training sessions per week, with each training session consisting of continuous cycling at an intensity of $\sim 70 \%$ HR maximum and lasting between 30-40 minutes. In addition to attending three supervised sessions per week, participants within the moderate-intensity continuous training group were also asked to perform two unsupervised moderateintensity sessions per week. Whilst it should be noted that this study primarily focused on and assessed cardiometabolic health, various questionnaires assessing psychological health were also completed at baseline and post-intervention (Bostic, McGartland, \& Hood, 2000; Diener, Emmons, Larsen, \& Griffin, 1985; Gauvin \& Rejeski, 1993; Ware \& Sherbourne, 2015; Watson, Clark, \& Tellegen, 1988). Shepherd et al. (2015) found that, in addition to improving cardiometabolic risk factors, HIIT significantly enhanced psychological well-being, with benefits similar to those achieved in the moderate-intensity comparison group. These findings were irrespective of the HIIT group participating in fewer exercise sessions and therefore completing a markedly smaller total exercise time. However, the interpretation of these psychological findings are perhaps limited given that the measures adopted (Bostic et al., 2000; Diener et al., 1985; Gauvin \& Rejeski, 1993; Ware \& Sherbourne, 2015; Watson et al., 1988) do not directly measure anxiety or depression and are not commonly used within research.

In contrast to Shepherd et al. (2015), research within adolescents has suggested that HIIT does not appear to be an effective means of improving mental well-being 
(Costigan et al., 2016). In Costigan et al. (2016), sixty-five adolescents (mean age = $15.8 \pm 0.6$ years) were randomly allocated to either a HIIT aerobic exercise program, a HIIT resistance and aerobic exercise program or control group. The school-based exercise sessions were completed during participants' physical education lessons or during a lunch break and were led by the research team. These sessions consisted of 8-10 minutes of either cardiorespiratory-based exercises or a combination of cardiorespiratory and body weight resistance exercises, with a work to rest ratio of 30:30 seconds and were completed 3 times per week for 8 weeks. Using magnitudebased inferences to assess the effects of the intervention, whilst difference were reported for other psychological measures, Costigan et al. (2016) did not detect any clinically important difference to markers of depression or anxiety, measured via the Kessler Psychological Distress Scale (Andrews \& Slade, 2001). However, these findings are limited given the small sample size that was further reduced in size once the participants were allocated to their respective groups.

Despite the limitations, the lack of clinically important differences in Costigan et al. (2016) are somewhat surprising given the neurological benefits of HIIT on psychological well-being (Saanijoki et al., 2017). Nonetheless, considering research has shown that HIIT is able to acutely reduce distress and state anxiety in a healthy population (Helgadóttir, Forsell, \& Ekblom, 2015), the length of the intervention may have affected the variation between the studies (Costigan et al., 2016; Shepherd et al., 2015). Indeed, it could be hypothesised that an 8-week HIIT intervention in Costigan et al. (2016) may have been insufficient to elicit enhancements to mental well-being, whereas, the longer intervention duration (10 weeks) utilised in Shepherd et al. (2015) may have been adequate to enhance mental health parameters. Given the abundance of literature supporting the effectiveness of HIIT in eliciting significant improvements in physiological health (Costigan, Eather, Plotnikoff, Taaffe, \& Lubans, 2015; Logan et al., 2014), the dearth of studies that have examined its effect on mental well-being is stark and warrants further research, especially considering the contradictory findings between both studies to date (Costigan et al., 2016; Shepherd et al., 2015).

\section{Overall summary}

Considering the importance of a healthy mental state and a good QoL, in conjunction with the reported decline of such health and relative paucity of studies in the general child and adolescent population, further research is critical. Within this youth 
population, methods to ameliorate such poor health are especially important given that mental health issues can track into later life. Therefore, studies should aim to prevent mental health issues rather than treating the symptoms, especially considering the purported greater long-term effectiveness. Given the large body of evidence supportive of increased physical activity and/or exercise as means to improve mental health and QoL, the effectiveness of HIIT is promising. Indeed, although previous research has suggested that HIIT does not improve mental health, methodological restrictions perhaps confounded these findings. Therefore, further research is needed to elucidate the effect of HIIT on mental health and QoL. 


\section{Chapter 3 - Effects of high-intensity interval training on physiological health in children and adolescents}

Whilst vigorous physical activity (VPA) has been shown to significantly improve mental health (Gerber et al., 2014; Parfitt et al., 2009), there remains a paucity of research exploring the effects of HIIT, the structured form of VPA, on mental wellbeing and QoL. Indeed, only two studies to date have assessed whether HIIT can effect mental health (Costigan et al., 2016; Shepherd et al., 2015). Consequently, conducting a systematic review and establishing an optimal protocol based on psychological literature was not possible and, therefore, an alternative approach to forming a suitable exercise protocol was required. As such, given the wealth of literature looking at physiological health (Rosenkranz et al., 2012; Tjønna et al., 2009; K. L. Weston et al., 2016), a systematic review was conducted to examine HIIT as a means of influencing key physiological health parameters. The identification of the most effective HIIT protocol was utilised for the intervention (Chapter 5).

The following chapter has been published as an original article in a peer-reviewed journal (Eddolls, W. T. B., McNarry, M. A., Stratton, G., Winn, C. O. N., \& Mackintosh, K. A. (2017). High-intensity interval training interventions in children and adolescents: a systematic review. Sports Medicine, 47(11), 2363-2374). With the exception of some minor amendments, it has been presented in its published form. The fully published version of this systematic review can be found in Appendix A (p. 178189).

\section{Introduction}

Worldwide, the prevalence of childhood and adolescent obesity have reached unparalleled levels (Lobstein et al., 2015; Ng et al., 2014). Specifically, in the UK, approximately $28 \%$ of children are classified as overweight or obese ( $\mathrm{Ng}$ et al., 2014; Ryley, 2013), representing a significant burden on health services. Current estimates suggest that obesity and its deleterious health consequences, such as diabetes mellitus type II (American Diabetes Association, 2000) and coronary heart disease (American Heart Association, 1997), cost the National Health Service £5.1 billion per year (Scarborough et al., 2011). Despite some reports suggest a plateau in paediatric obesity over the last decade (Rokholm et al., 2010), others suggest that UK obesity levels have increased between 1980 and 2014 by $48 \%$ and 39\% in boys and girls, respectively ( $\mathrm{Ng}$ 
et al., 2014). Of concern, paediatric obesity has been associated with an increased prevalence of cardiometabolic risk factors (Skinner, Perrin, Moss, \& Skelton, 2015), which have been shown to track into later life (Freedman et al., 2007) and increase the risk of premature mortality (Franks et al., 2010; Freedman et al., 2007). While the causes of obesity and cardiometabolic risk are multifaceted, low physical activity levels, as well as high engagement in sedentary pursuits, have been identified as key contributory factors (Freedman et al., 2007; Janssen et al., 2005; Kopelman, 2000; Trost et al., 2001). Specifically, according to the latest statistics, in England, only $21 \%$ of boys and $16 \%$ of girls, meet UK physical activity guidelines of at least 60 minutes of moderate-to-vigorous physical activity every day (Ryley, 2013), with physical activity levels further declining with age (Heitzler et al., 2011; Sallis, Prochaska, \& Taylor, 2000; Troiano et al., 2008). Effective interventions targeted at increasing youth physical activity levels are therefore imperative.

While traditional interventions designed to increase physical activity and improve health have principally utilised moderate-intensity, continuous exercise (Hansen et al., 1991; McMurray et al., 2002; Meyer et al., 2006), the relevance of such programmes to the sporadic, high-intensity nature of children's habitual play patterns has been questioned (Bailey et al., 1995). Consequently, high-intensity interval-based programmes have recently been investigated as a potentially effective and timeefficient form of physical activity and health promotion (Gibala, 2007). Whilst exercise, a sub-component of physical activity, is structured and conducted for the health-associated benefits, it could provide a necessary mediatory step in order to provoke positive long-term behaviour change. However, to date, studies have demonstrated varied success at eliciting significant improvements (Baquet et al., 2002; Baquet, Berthoin, Gerbeaux, \& van Praagh, 2001; Lau et al., 2014; Tjønna et al., 2009), potentially due to a lack of consensus regarding an optimum HIIT intervention protocol with regard to exercise intensity, frequency and duration. Nonetheless, recent systematic reviews (Costigan, Eather, Plotnikoff, Taaffe, \& Lubans, 2015; Logan et al., 2014) highlighted that HIIT can elicit greater improvements in health-related parameters (i.e., cardiometabolic health and body composition) in adolescents, compared to traditional programmes (Racil et al., 2016; Rosenkranz et al., 2012; Tjønna et al., 2009; K. L. Weston et al., 2016). However, no systematic reviews have investigated the effects of HIIT on primary school-aged children, the identified 
potential differences between pre-pubertal and pubertal youth in the adaptations elicited or provided optimal HIIT protocol recommendations. Therefore, the purpose of this review was to systematically synthesise the scientific literature regarding HIIT on improving body composition, cardiometabolic health and cardiovascular health in children and adolescents and to establish an optimal HIIT protocol with regard to session structure, intensity, frequency and duration.

\section{Methodology}

In line with the Preferred Reporting Items for Systematic Reviews and Meta-Analyses (PRISMA) (Liberati et al., 2009), the following methodology details the review's inclusion criteria, search strategy, data collection and study analysis protocols.

\section{Inclusion criteria}

\section{Types of study}

The present review included studies involving interventions targeted at reducing obesity-related physiological parameters with a principal focus on one of the following: high-intensity physical activity, high-intensity exercise/training or highintensity intermittent/interval exercise/training interventions.

\section{Types of participant}

Studies incorporating children and adolescents between the ages of 5 and 18 years were included. These age constraints were applied to all children and adolescents without disability, irrespective of whether they were a healthy weight, overweight or obese. Studies that utilised physical activity interventions as part of a treatment for specific illnesses were excluded.

\section{Intervention variables and outcome measures}

To be included in the review, studies were required to report a minimum of one intervention exercise session intensity variable and one outcome measure, measured at baseline and post-intervention and compared to either a moderate-intensity exercise intervention or control group.

\section{Intervention intensity variables}

Interventions were defined as high-intensity if: i) the intensity was greater than or equal to $90 \%$ peak oxygen uptake ( $\dot{V} \mathrm{O}_{2 \text { peak}}$; Gibala \& McGee, 2008); ii) had an 
intensity that was equal to or greater than $100 \%$ maximal aerobic speed (MAS; Baker, 2011); and/or iii) ensured that the participant's HR was equal to or greater than $90 \%$ of their peak HR (Hood et al., 2011; Little et al., 2011). There were no restrictions applied regarding the duration of the intervention.

\section{Primary outcomes}

Primary outcomes included cardiometabolic health markers, namely mean SBP and DBP, body composition in the form BMI, BF\% and fat-free mass (FFM), and cardiovascular disease biomarker analysis that include at least one of the following: glucose, insulin, triglyceride and total cholesterol, as well as its sub-fractions, LDLcholesterol and HDL-cholesterol.

\section{Search strategy}

Electronic databases were searched until September 2016, with no restriction set on the publication year. The PubMed and SCOPUS databases were explored using the following key word search strategy, devised by the research team and verified by a subject librarian: (high intensity training OR high intensity exercise* OR high intensity activit* OR high intensity intermittent training OR intensity intermittent exercise* OR high intensity intermittent activit* or high intensity interval training OR high intensity interval exercise* OR high intensity interval activit*) AND (child* OR children OR pediatric OR paediatric OR adolescen* OR juvenile*) AND (health OR healthy). Inclusion of at least one of the key words was required in the study title for it to be considered. Studies were excluded based on language; only studies written in English were included. Additional studies were identified by searching the reference lists of included studies. Google Scholar and ResearchGate were also searched to identify studies that were potentially overlooked by the database searches.

\section{Data collection and analysis}

Pertinent study abstracts from the stated search strategies were downloaded and independently screened. Studies that were deemed to meet the criteria were downloaded in their entirety and independently assessed for relevance by two authors using the pre-determined criteria. When study information was missing, the research team attempted to contact the primary author of the incomplete study. If the author failed to respond, the study was excluded. 


\section{Effect Size}

Cohen's d was used to determine the standardised mean effect of HIIT on the previously outlined health-related outcome measures compared to a control group or a moderate- or light-intensity group (J. Cohen, 1988). Confidence intervals (CI) were calculated by applying an equation recommended by Nakagawa and Cuthill (2007), employing standard error calculations (Hunter \& Schmidt, 2004). For studies that provided values for both moderate and control groups, moderate group values were included as the comparison. Additionally, effect size was not calculated for studies that failed to disclose post-intervention mean values.

\section{Risk of bias assessment}

Risk of bias was assessed independently by two reviewers using the Cochrane risk of bias tool (RoB 2.0; Higgins et al., 2016). Using the RoB 2.0 tool, studies were awarded an overall risk of bias grade of either high, some or low risk of bias. This overall grade was calculated by assessing five domains: (A) bias arising from the randomisation process; (B) bias due to deviations from intended interventions; (C) bias due to missing outcome data; (D) bias in measurement of the outcome; and (E) bias in selection of the reported result.

\section{Heterogeneity assessment}

Due to the variation of the study characteristics in this review, for example between interventions, outcome measures and cohort populations, it was deemed unsuitable to amalgamate the results for a meta-analysis. Therefore, the results in this review were analysed narratively.

\section{Results}

The database search generated 2092 studies. Once duplicates were removed, 54 title/abstracts were screened for eligibility, with the reference list search producing 3 further studies. From this, 13 studies were deemed suitable. The screening process is shown in Figure 3.1. In total, 7 of the 13 studies included in this review examined the effects of HIIT interventions on pre-pubertal participants, the characteristics of which are summarised in Table 3.1, with the remaining 6 studies examining the effects of HIIT interventions on pubertal participants (Table 3.2). 
Records identified through database
searching
$(\mathrm{n}=2092)$
Additional records identified through other sources

$(\mathrm{n}=3)$

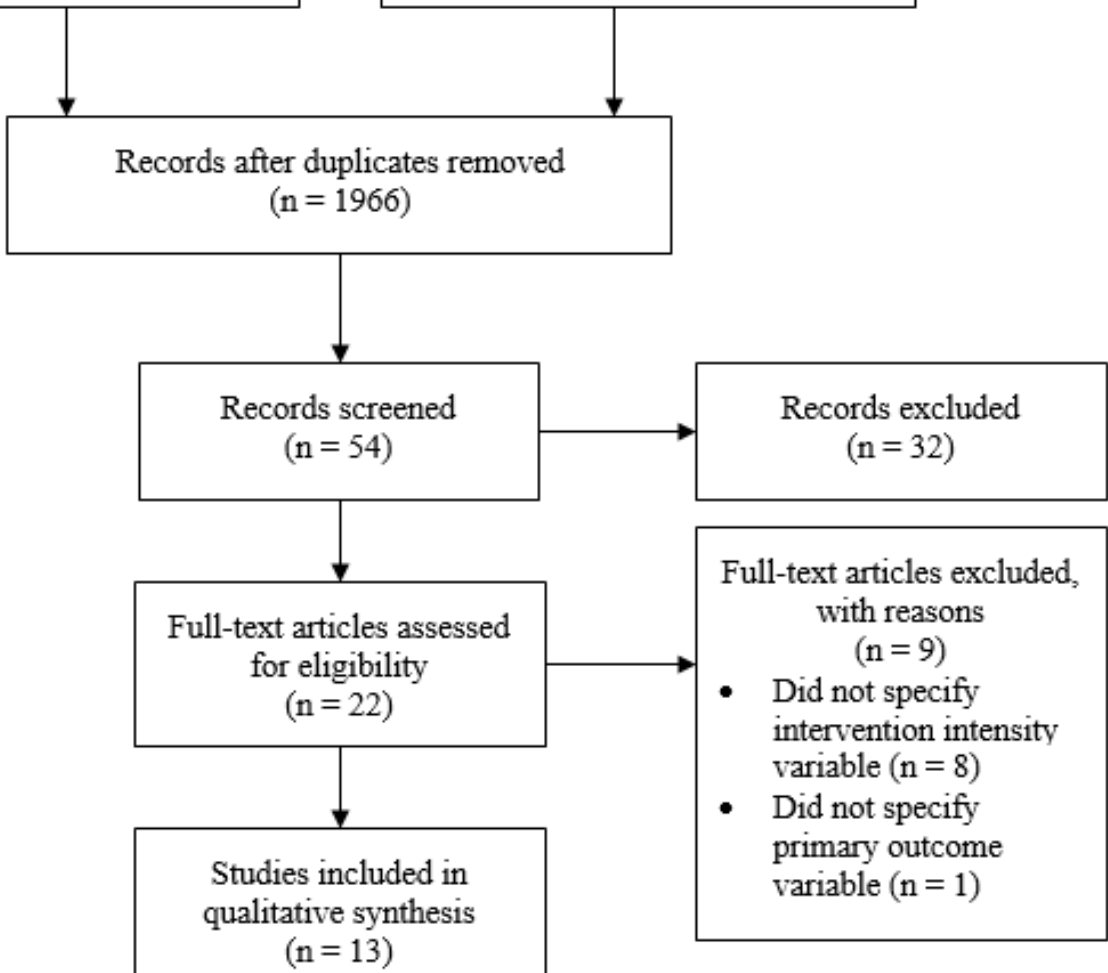

Figure 3.1 Phases of study selection during data collection 
Table 3.1 Characteristics of studies examining pre-pubertal participants

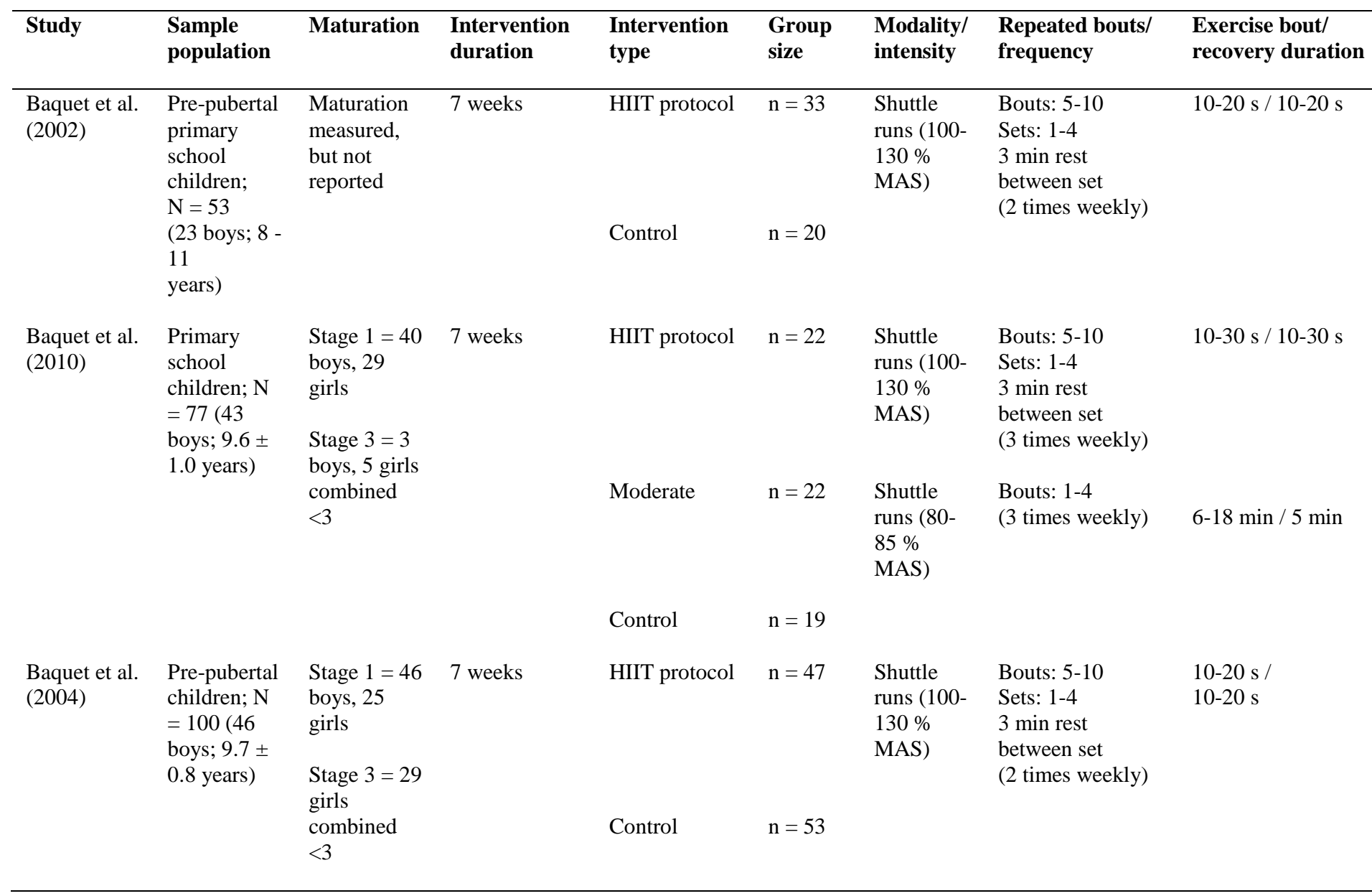




\begin{tabular}{|c|c|c|c|c|c|c|c|c|}
\hline $\begin{array}{l}\text { Lambrick et al. } \\
\text { (2016) }\end{array}$ & $\begin{array}{l}\text { Obese and } \\
\text { normal } \\
\text { weight } \\
\text { children; } \mathrm{N} \\
=55(32 \\
\text { boys) }\end{array}$ & $\begin{array}{l}\text { Peak height } \\
\text { velocity at } \\
\text { baseline: } \\
\text { INT }=2.7 \pm \\
0.1 \\
\text { CON }=2.65 \\
\pm 0.05\end{array}$ & 6 weeks & HIIT Protocol & $\mathrm{n}=28$ & $\begin{array}{l}\text { Child } \\
\text { specific } \\
\text { games } \\
(93 \% \\
\text { mean } \\
\left.\text { HR }_{\max }\right)\end{array}$ & $\begin{array}{l}\text { Bouts: } 7 \\
\text { (2 times weekly) }\end{array}$ & $6 \mathrm{~min} / 2 \mathrm{~min}$ \\
\hline \multirow[t]{3}{*}{ Lau et al. (2014) } & $\begin{array}{l}\text { Overweight } \\
\text { primary } \\
\text { school } \\
\text { children; N }\end{array}$ & $\begin{array}{l}\text { Maturation } \\
\text { not reported }\end{array}$ & 6 weeks & HIIT protocol & $\mathrm{n}=15$ & $\begin{array}{l}\text { Shuttle } \\
\text { runs }(120 \\
\% \text { MAS })\end{array}$ & $\begin{array}{l}\text { Bouts: } 12 \\
\text { ( } 3 \text { times weekly) }\end{array}$ & $15 \mathrm{~s} / 15 \mathrm{~s}$ \\
\hline & $\begin{array}{l}=48(36 \\
\text { boys; } 10.4 \pm \\
0.9 \text { years })\end{array}$ & & & Light & $\mathrm{n}=21$ & $\begin{array}{l}\text { Shuttle } \\
\text { runs }(100 \\
\% \text { MAS })\end{array}$ & $\begin{array}{l}\text { Bouts: } 16 \\
\text { ( } 3 \text { times weekly) }\end{array}$ & $15 \mathrm{~s} / 15 \mathrm{~s}$ \\
\hline & & & & Control & $\mathrm{n}=12$ & & & \\
\hline \multirow[t]{2}{*}{$\begin{array}{l}\text { Nourry et al. } \\
\text { (2005) }\end{array}$} & $\begin{array}{l}\text { Pre-pubertal } \\
\text { children; } \mathrm{N} \\
=18(11 \\
\text { boys; } 10.0 \pm \\
0.8 \text { years })\end{array}$ & Stage $1=18$ & 8 weeks & HIIT protocol & $\mathrm{n}=9$ & $\begin{array}{l}\text { Shuttle } \\
\text { runs (100- } \\
130 \% \\
\text { MAS) }\end{array}$ & $\begin{array}{l}\text { Bouts: } 10 \\
\text { Sets: } 4 \text { times } \\
\text { ( } 2 \text { times weekly) }\end{array}$ & $10-20 \mathrm{~s} / 10-20 \mathrm{~s}$ \\
\hline & & & & Control & $\mathrm{n}=9$ & & & \\
\hline \multirow[t]{2}{*}{$\begin{array}{l}\text { Rosenkranz et al. } \\
\text { (2012) }\end{array}$} & $\begin{array}{l}\text { Pre-pubertal } \\
\text { children; N } \\
=16(2 \\
\text { boys; } 7-12 \\
\text { years })\end{array}$ & Stage $1=16$ & 8 weeks & HIIT protocol & $\mathrm{n}=8$ & $\begin{array}{l}\text { Shuttle } \\
\text { runs }(100- \\
130 \% \\
\text { MAS) }\end{array}$ & $\begin{array}{l}\text { Bouts: } 5-10 \\
\text { Sets: } 4 \text { times } \\
\text { ( } 2 \text { times weekly) }\end{array}$ & $10-20 \mathrm{~s} / 10-20 \mathrm{~s}$ \\
\hline & & & & Control & $\mathrm{n}=8$ & & & \\
\hline
\end{tabular}

HIIT high-intensity interval training, MAS maximal aerobic speed, INT intervention group, $\mathrm{HR}_{\max }$ heart rate 
Table 3.2 Characteristics of studies examining pubertal participants

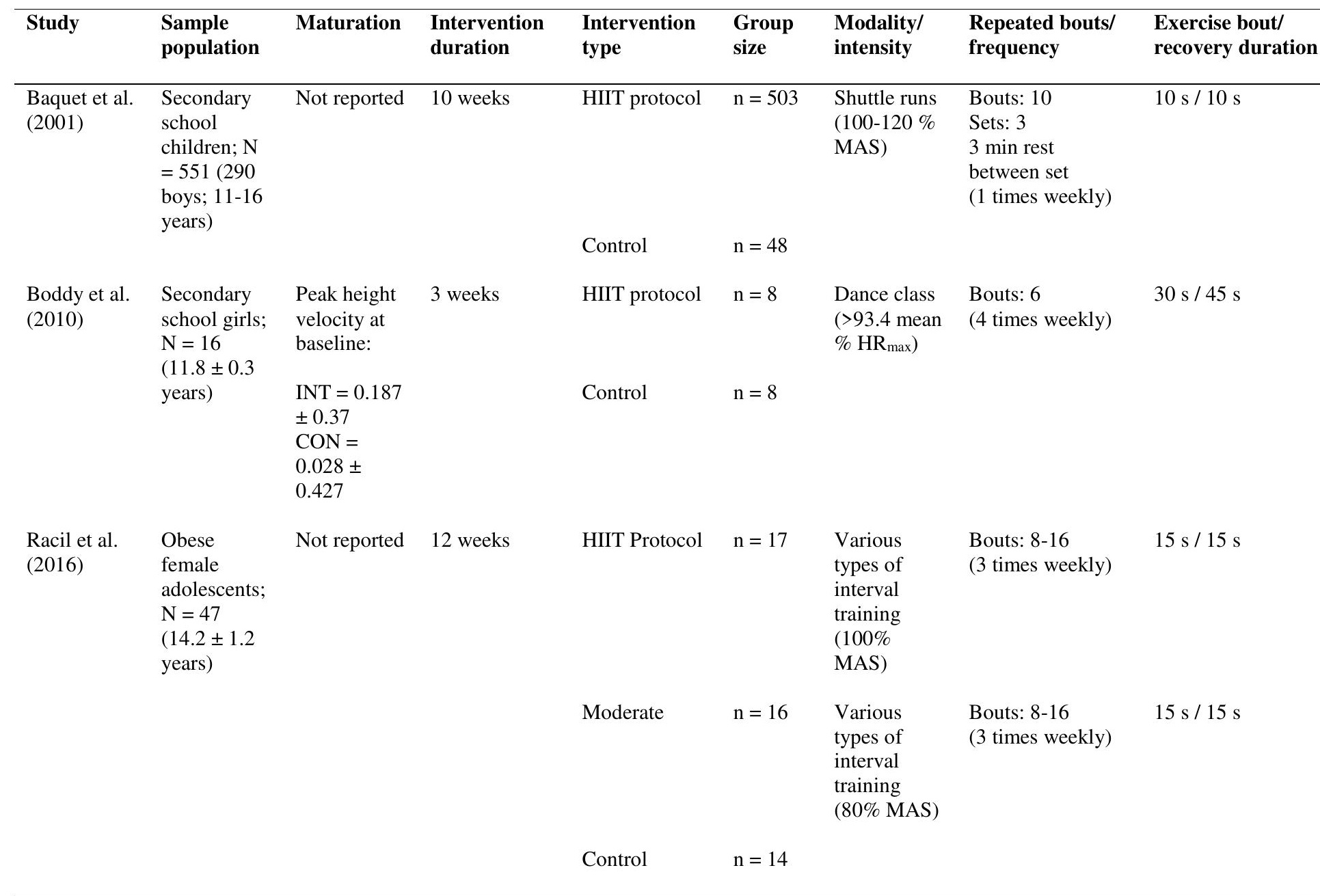




\begin{tabular}{|c|c|c|c|c|c|c|c|c|}
\hline \multirow[t]{2}{*}{$\begin{array}{l}\text { Sperlich et al. } \\
(2011)\end{array}$} & \multirow[t]{2}{*}{$\begin{array}{l}\text { Male soccer } \\
\text { players; } \mathrm{N}= \\
19(13.5 \pm \\
0.4 \text { years })\end{array}$} & \multirow[t]{2}{*}{$\begin{array}{l}\text { Maturation } \\
\text { not reported }\end{array}$} & \multirow[t]{2}{*}{5 weeks } & HIIT protocol & $\mathrm{n}=9$ & $\begin{array}{l}\text { Various } \\
\text { types of } \\
\text { interval } \\
\text { training } \\
(90-95 \% \\
\left.\mathrm{HR}_{\max }\right)\end{array}$ & $\begin{array}{l}\text { Bouts: } 4-12 \\
\text { (3-4 times } \\
\text { weekly) }\end{array}$ & $\begin{array}{l}30 \mathrm{~s}-4 \mathrm{~min} / 30 \\
3 \mathrm{~min}\end{array}$ \\
\hline & & & & Moderate & $\mathrm{n}=8$ & $\begin{array}{l}\text { Various } \\
\text { types of } \\
\text { interval } \\
\text { training } \\
(50-70 \% \\
\left.\text { HR }_{\max }\right)\end{array}$ & $\begin{array}{l}\text { Bouts: } 1-5 \\
\text { (3-4 times } \\
\text { weekly) }\end{array}$ & $10-30 \mathrm{~min} / 1-3$ \\
\hline \multirow[t]{2}{*}{ Tjønna et al. (2009) } & 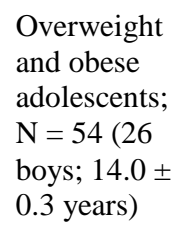 & $\begin{array}{l}\text { Maturation } \\
\text { not reported }\end{array}$ & 3 months & HIIT protocol & $\mathrm{n}=28$ & $\begin{array}{l}\text { Treadmill } \\
\text { walking/r } \\
\text { unning } \\
(90-95 \% \\
\left.\text { HR }_{\max }\right)\end{array}$ & $\begin{array}{l}\text { Bouts: } 4 \\
\text { (2 times weekly) }\end{array}$ & $4 \mathrm{~min} / 3 \mathrm{~min}$ \\
\hline & & & & Control & $\mathrm{n}=26$ & & & \\
\hline $\begin{array}{l}\text { K. L. Weston et al. } \\
\text { (2016) }\end{array}$ & $\begin{array}{l}\text { Adolescent } \\
\text { secondary } \\
\text { school } \\
\text { children; } \mathrm{N} \\
=101(62 \\
\text { boys; } 14.0 \pm \\
0.3 \text { years })\end{array}$ & $\begin{array}{l}\text { Peak height } \\
\text { velocity at } \\
\text { baseline: } \\
\text { INT }=0.3 \pm \\
1.0 \\
\text { Control }= \\
0.5 \pm 1.3\end{array}$ & 10 weeks & HIIT Protocol & $\mathrm{n}=60$ & $\begin{array}{l}\text { Games } \\
\text { based } \\
(>90 \% \\
\text { mean } \\
\left.\mathrm{HR}_{\max }\right)\end{array}$ & $\begin{array}{l}\text { Bouts: } 4-7 \\
\text { (3 times weekly) }\end{array}$ & $45 \mathrm{~s} / 90 \mathrm{~s}$ \\
\hline
\end{tabular}

INT intervention, HIIT high-intensity interval training, MAS maximal aerobic speed, INT intervention grou 


\section{Risk of bias}

The methodological rigour of studies included in this review, according to the risk of bias assessment, is presented in Table 3.3. Seven studies were considered to have a high risk of bias (Baquet et al., 2002, 2010, 2001, 2004; Lau et al., 2014; Sperlich et al., 2011; K. L. Weston et al., 2016), whereas only two (Nourry et al., 2005; Rosenkranz et al., 2012) and four (Boddy et al., 2010; Lambrick et al., 2016; Racil et al., 2016; Tjønna et al., 2009) studies were considered to have some or low risk of bias, respectively. In studies deemed to have a high or some risk of bias (Baquet et al., 2002, 2010, 2001, 2004; Lau et al., 2014; Nourry et al., 2005; Rosenkranz et al., 2012; Sperlich et al., 2011; K. L. Weston et al., 2016), the bias arose from the randomisation process (Domain A).

Table 3.3 Risk of bias assessment

\begin{tabular}{lcccccc}
\hline Study & $\begin{array}{c}\text { Domain } \\
\text { A }\end{array}$ & $\begin{array}{c}\text { Domain } \\
\text { B }\end{array}$ & $\begin{array}{c}\text { Domain } \\
\text { C }\end{array}$ & $\begin{array}{c}\text { Domain } \\
\text { D }\end{array}$ & $\begin{array}{c}\text { Domain } \\
\text { E }\end{array}$ & Total \\
\hline Baquet et al. (2002) & High & Low & Low & Low & Low & High \\
Baquet et al. (2001) & High & Low & Low & Low & Low & High \\
Baquet et al. (2010) & High & Low & Low & Low & Low & High \\
Baquet et al. (2004) & High & Low & Low & Low & Low & High \\
Boddy et al. (2010) & Low & Low & Low & Low & Low & Low \\
Lambrick et al. (2016) & Low & Low & Low & Low & Low & Low \\
Lau et al. (2014) & High & Low & Low & Low & Low & High \\
Nourry et al. (2005) & Some & Low & Low & Low & Low & Some \\
Racil et al. (2016) & Low & Low & Low & Low & Low & Low \\
Rosenkranz et al. (2012) & Some & Low & Low & Low & Low & Some \\
Sperlich et al. (2011) & High & Low & Low & Low & Low & High \\
Tjønna et al. (2009) & Low & Low & Low & Low & Low & Low \\
K. L. Weston et al. (2016) & High & Low & Low & Low & Low & High \\
\hline
\end{tabular}

Domain: (A) bias arising from the randomisation process, (B) bias due to deviations from intended interventions, (C) bias due to missing outcome data, (D) bias in measurement of the outcome, (E) bias in selection of the reported result, Total overall risk of bias grade calculated by assessing the five domains [A-E]

\section{Body mass and composition}

All 13 included studies reported the effect of HIIT compared to moderate-intensity exercise or a control group on BMI $(n=9), \mathrm{BF} \%(\mathrm{n}=9)$ or FFM $(\mathrm{n}=1)$. The results revealed little evidence to suggest that HIIT can elicit significant changes in body composition (Table 3.4), although, Tjønna et al. (2009) and Racil et al. (2016), both of which were assessed to be at low risk of bias, reported significant improvements in 
Table 3.4 Baseline and post-intervention changes to body mass/composition and effect size between HII

\begin{tabular}{|c|c|c|c|c|}
\hline Study & $\begin{array}{l}\text { Outcome } \\
\text { measure }\end{array}$ & $\begin{array}{c}\text { HIIT (mean } \\
\text { change from } \\
\text { baseline) }\end{array}$ & $\begin{array}{l}\text { Control/moderate } \\
\text { (mean change } \\
\text { from baseline) }\end{array}$ & $\begin{array}{l}\text { Effect s } \\
\text { (Cohen' }\end{array}$ \\
\hline Baquet et al. (2001) & $\mathrm{BMI}\left(\mathrm{kg} \cdot \mathrm{m}^{-2}\right)$ & $0.40 * *$ & $0.60 * *$ & -0.14 \\
\hline Baquet et al. (2010) & $\mathrm{BMI}\left(\mathrm{kg} \cdot \mathrm{m}^{-2}\right)$ & 0.10 & $-0.30 / 0.20$ & -0.10 \\
\hline Boddy et al. (2010) & $\mathrm{BMI}\left(\mathrm{kg} \cdot \mathrm{m}^{-2}\right)$ & -0.50 & 0.20 & 0.93 \\
\hline Lambrick et al. (2016) & $\mathrm{BMI}\left(\mathrm{kg} \cdot \mathrm{m}^{-2}\right)$ & 0.00 & 0.00 & 0.23 \\
\hline Lau et al. (2014) & $\mathrm{BMI}\left(\mathrm{kg} \cdot \mathrm{m}^{-2}\right)$ & 0.20 & $0.10 / 0.40 *$ & 0.42 \\
\hline Racil et al. (2016) & $\mathrm{BMI}\left(\mathrm{kg} \cdot \mathrm{m}^{-2}\right)$ & $-3.20 *$ & $0.30 /-1.70 *$ & -1.41 \\
\hline Rosenkranz et al. (2012) & $\operatorname{BMI}\left(\mathrm{kg} \cdot \mathrm{m}^{-2}\right)$ & -1.40 & 0.00 & -0.06 \\
\hline Tjønna et al. (2009) & $\mathrm{BMI}\left(\mathrm{kg} \cdot \mathrm{m}^{-2}\right)$ & $-0.70 * *$ & -0.20 & -1.50 \\
\hline K. L. Weston et al. (2016) & $\mathrm{BMI}\left(\mathrm{kg} \cdot \mathrm{m}^{-2}\right)$ & $-0.60^{\mathrm{a}}$ & $0.80^{\mathrm{a}}$ & N/A \\
\hline Baquet et al. (2001) & Body fat (\%) & $1.60 *$ & $1.30 *$ & -0.31 \\
\hline Baquet et al. (2002) & Body fat (\%) & -0.90 & -0.70 & 0.10 \\
\hline Baquet et al. (2004) & Body fat (\%) & 0.10 & 0.10 & 0.02 \\
\hline Boddy et al. (2010) & Body fat (\%) & 0.22 & 0.46 & 0.61 \\
\hline Lambrick et al. (2016) & Body fat (\%) & -0.10 & 0.40 & 0.08 \\
\hline Nourry et al. (2005) & Body fat (\%) & 1.50 & 0.50 & 0.14 \\
\hline Racil et al. (2016) & Body fat (\%) & $-3.90 *$ & $-0.50 /-3.40 *$ & -0.59 \\
\hline Rosenkranz et al. (2012) & Body fat (\%) & -2.20 & -1.00 & -0.17 \\
\hline Tjønna et al. (2009) & Body fat (\%) & $-0.90 * *$ & -0.30 & 3.00 \\
\hline Sperlich et al. (2011) & FFM (kg) & 1.00 & 0.90 & 0.71 \\
\hline
\end{tabular}

Significantly different from baseline $\left({ }^{*} \mathrm{p}<0.05,{ }^{* *} \mathrm{p}<0.01\right),{ }^{\mathrm{a}}$ values adjusted for sex, baseline value and maturi calculated due to no reported post-intervention means, HIIT high-intensity interval training group, CI confide FFM fat-free mass 
BMI and BF\% associated with a medium-to-large effect sizes following a 3-month intervention.

It is pertinent to note that while other studies failed to find a significant improvement in measures of body mass or composition, there was a general trend for greater change in body mass and composition in the HIIT group (Baquet et al., 2002; Nourry et al., 2005; Rosenkranz et al., 2012; Sperlich et al., 2011; K. L. Weston et al., 2016). The exception to this was Baquet et al. (2001), who saw significant increases to BMI and $\mathrm{BF} \%$ for both the HIIT protocol and the control group, however, this study was deemed to have a high risk of bias. While no significant benefits were reported in pre-pubertal children, significant improvements in body mass and composition have been demonstrated in pubertal children (Racil et al., 2016; Tjønna et al., 2009), suggesting a potential maturational effect. There were insufficient data to examine potential sex differences regarding the efficacy of HIIT in eliciting significant changes in body composition.

\section{Cardiovascular health}

\section{Blood pressure}

Five studies investigated the effect of HIIT on SBP and DBP, with the majority concluding significant benefits were obtained (Table 3.5). Specifically, two studies that were assessed to be of low risk of bias, Tjønna et al. (2009) and Racil et al. (2016), reported significant improvements in both SBP and DBP following the intervention, although it is pertinent to note the low effect sizes associated with the improvements reported for SBP and DBP in Racil et al. (2016; Table 3.5). Whilst the remaining studies (Boddy et al., 2010; Rosenkranz et al., 2012; K. L. Weston et al., 2016) reported no significant differences, they demonstrated a trend towards a lower SBP and DBP. Interestingly, Boddy et al. (2010), demonstrating methodological rigour through a low risk of bias, found an increase in both DBP and SBP in the HIIT group and reductions in the control group, though not significant. Regarding maturation differences, no studies examining pre-pubertal children reported significant changes, whereas significant improvements were reported in pubertal populations (Racil et al., 2016; Tjønna et al., 2009). Sex differences could not be investigated due to insufficient data. 
Table 3.5 Baseline to post-intervention changes in SBP/DBP and effect size between HIIT and co

\begin{tabular}{lcccr}
\hline Study & $\begin{array}{c}\text { Outcome } \\
\text { measure }\end{array}$ & $\begin{array}{c}\text { HIIT (mean } \\
\text { change from } \\
\text { baseline) }\end{array}$ & $\begin{array}{c}\text { Control/moderate } \\
\text { (mean change } \\
\text { from baseline) }\end{array}$ & $\begin{array}{c}\text { Effect } \\
\text { (Cohen }\end{array}$ \\
\hline Boddy et al. (2010) & SBP (mmHg) & 10.10 & -1.40 & 0.34 \\
Racil et al. (2016) & SBP (mmHg) & $-0.60^{*}$ & $0.00 /-0.40^{*}$ & 0.00 \\
Rosenkranz et al. (2012) & SBP (mmHg) & -2.20 & -2.50 & -0.5 \\
Tjønna et al. (2009) & SBP (mmHg) & $-9.40^{* *}$ & $-2.50^{*}$ & -2.0 \\
K. L. Weston et al. (2016) & SBP (mmHg) & $-5.00^{\mathrm{a}}$ & $-1.00^{\mathrm{a}}$ & $\mathrm{N} / \mathrm{A}$ \\
& & & & \\
Boddy et al. (2010) & $\mathrm{DBP}(\mathrm{mmHg})$ & 5.90 & -4.10 & 1.14 \\
Racil et al. (2016) & $\mathrm{DBP}(\mathrm{mmHg})$ & $-6.00^{*}$ & $-1.00 /-4.00^{*}$ & -0.3 \\
Rosenkranz et al. (2012) & $\mathrm{DBP}(\mathrm{mmHg})$ & -2.50 & -1.70 & -0.8 . \\
Tjønna et al. (2009) & $\mathrm{DBP}(\mathrm{mmHg})$ & $-5.50^{* *}$ & 1.80 & -1.5 \\
K. L. Weston et al. (2016) & $\mathrm{DBP}(\mathrm{mmHg})$ & $-6.00^{\mathrm{a}}$ & $-4.00^{\mathrm{a}}$ & $\mathrm{N} / \mathrm{A}$ \\
\hline
\end{tabular}

Significantly different from baseline $\left({ }^{*} \mathrm{p}<0.05,{ }^{*} \mathrm{p}<0.01\right),{ }^{\mathrm{a}}$ values adjusted for sex, baseline value and maturi calculated due to no reported post-intervention means, HIIT high-intensity interval training group, CI confide pressure, DBP diastolic blood pressure 


\section{Cardiovascular disease biomarker health}

Four studies examined the effect of HIIT on cardiovascular disease biomarkers, specifically blood glucose $(n=4)$, total cholesterol $(n=2)$, HDL-cholesterol $(n=3)$, LDL-cholesterol $(n=1)$, blood triglyceride $(n=3)$ and insulin $(n=2)$, concluding that HIIT is an effective strategy for such improvements (Table 3.6). All of the studies demonstrated significant (Racil et al., 2016; Rosenkranz et al., 2012; Tjønna et al., 2009) or clinically substantial (K. L. Weston et al., 2016) improvements in multiple cardiovascular disease biomarker outcome measures. Tjønna et al. (2009) reported significant improvements for blood glucose in favour of the HIIT group $(p<0.01)$ compared to the control group $(p<0.05)$, with an effect size indicating large, clinically important differences ( $d=-1.43,95 \% \mathrm{CI}-3.01$ to 0.16$)$. Additionally, results from Rosenkranz et al. (2012) suggest large significant reductions in total cholesterol compared to the control group. Further, the associated effect size $(d=-0.93,95 \% \mathrm{CI}$ 1.16 to -0.70 ) suggested highly clinically important differences. Rosenkranz et al. (2012) saw significant reductions $(p<0.05)$ in LDL-cholesterol as a result of HIIT, with a large and clinically important difference $(d=-1.67,95 \%$ CI -2.03 to -1.31$)$ compared to a small, non-significant increase in the control group. Examining blood insulin results in Tjønna et al. (2009), larger significant reductions in the HIIT group were found compared to the control group, with further significant reductions after a 12-month follow-up. In addition, HDL-cholesterol significantly improved in the HIIT group compared to a non-significant increase in the control group. Furthermore, Racil et al. (2016) reported significant reductions in blood glucose and insulin in both HIIT and moderate-intensity protocols compared to a control group, with a greater improvement reported in the HIIT group for both measures. Effect sizes for both blood glucose and insulin were low-to-moderate $(d=0.32,95 \% \mathrm{CI}-0.44$ to 0.13$)$ and large $(d=-0.82,95 \%$ CI -1.55 to -0.10$)$, respectively. Notwithstanding the finite improvement in blood triglycerides, K. L. Weston et al. (2016) reported clinically substantial beneficial effects as a result of HIIT despite increased triglyceride levels reported in the control group. Despite these encouraging findings, it is pertinent to note that the studies by Rosenkranz et al. (2012) and K. L. Weston et al. (2016) were assessed to have 'some' and a 'high' risk of bias, respectively, therefore, caution should be taken when interpreting these studies as methodological limitations may have confounded the results. There was no effect due to maturation on cardiovascular 
disease biomarkers, with significant or clinically substantial improvements found in both pre-pubertal (Rosenkranz et al., 2012) and pubertal (Racil et al., 2016; Tjønna et al., 2009; K. L. Weston et al., 2016) children. Sex differences were not reported and, therefore, their effect is unknown.

\section{HIIT protocol}

All studies included in this review provided a detailed description of their intervention protocol in terms of session structure, duration, intensity and frequency, in addition to intervention duration; key details of these are summarised in Table 3.1 and Table 3.2. Based on the four studies that demonstrated significant health improvements (Racil et al., 2016; Rosenkranz et al., 2012; Tjønna et al., 2009; K. L. Weston et al., 2016), a running-based HIIT intervention at an intensity of >90\% maximum HR/100-130 \% maximal aerobic velocity, 2-3 times a week with a minimum intervention duration lasting 7 weeks could be considered the suggested practice. However, suggested exercise session duration and rest intervals remain ambiguous due to the variance across the studies, a notion also supported by Baquet, van Praagh and Berthoin (2003). 
Table 3.6 Baseline and post-intervention changes in cardiovascular disease biomarkers and effect size betwe protocols.

\begin{tabular}{|c|c|c|c|}
\hline Study & Outcome measure & $\begin{array}{c}\text { HIIT (mean } \\
\text { change from } \\
\text { baseline) } \\
\end{array}$ & $\begin{array}{c}\text { Control/moderate } \\
\text { (mean change } \\
\text { from baseline) }\end{array}$ \\
\hline Racil et al. (2016) & Glucose $\left(\mathrm{mmol} \cdot \mathrm{l}^{-1}\right)$ & $-0.20^{*}$ & $0.00 /-0.20^{*}$ \\
\hline Rosenkranz et al. (2012) & Glucose $\left(\mathrm{mg} \cdot \mathrm{dl}^{-1}\right)$ & 5.20 & 0.40 \\
\hline Tjønna et al. (2009) & Glucose $\left(\mathrm{mmol} \cdot \mathrm{l}^{-1}\right)$ & $-0.30 * *$ & -0.10 \\
\hline K. L. Weston et al. (2016) & Glucose $\left(\mathrm{mmol} \cdot \mathrm{l}^{-1}\right)$ & $-0.10^{\mathrm{a}}$ & $-0.03^{\mathrm{a}}$ \\
\hline Rosenkranz et al. (2012) & Total cholesterol $\left(\mathrm{mg} \cdot \mathrm{dl}^{-1}\right)$ & $-22.00 *$ & 2.40 \\
\hline K. L. Weston et al. (2016) & Total cholesterol $\left(\mathrm{mmol} \cdot \mathrm{l}^{-1}\right)$ & $-0.24^{\mathrm{a}}$ & $0.00^{\mathrm{a}}$ \\
\hline Rosenkranz et al. (2012) & HDL-cholesterol $\left(\mathrm{mg} \cdot \mathrm{dl}^{-1}\right)$ & 9.90 & 3.60 \\
\hline Tjønna et al. (2009) & HDL-cholesterol $\left(\mathrm{mmol} \cdot \mathrm{l}^{-1}\right)$ & $0.11 *$ & 0.09 \\
\hline K. L. Weston et al. (2016) & HDL-cholesterol $\left(\mathrm{mmol} \cdot \mathrm{l}^{-1}\right)$ & $-0.14^{\mathrm{a}}$ & $-0.24^{\mathrm{a}}$ \\
\hline Rosenkranz et al. (2012) & LDL-cholesterol $\left(\mathrm{mg} \cdot \mathrm{dl}^{-1}\right)$ & $-34.80 *$ & -5.60 \\
\hline Rosenkranz et al. (2012) & Triglycerides $\left(\mathrm{mg} \cdot \mathrm{dl}^{-1}\right)$ & 23.50 & 3.50 \\
\hline Tjønna et al. (2009) & Triglycerides $\left(\mathrm{mmol} \cdot \mathrm{l}^{-1}\right)$ & -0.50 & -0.10 \\
\hline K. L. Weston et al. (2016) & Triglycerides $\left(\mathrm{mmol} \cdot \mathrm{l}^{-1}\right)$ & $-0.05^{\mathrm{a}}$ & $0.18^{\mathrm{a}}$ \\
\hline Racil et al. (2016) & Insulin $\left(\mathrm{IU} \cdot \mathrm{ml}^{-1}\right)$ & $-5.70^{*}$ & $-0.80 /-4.30 *$ \\
\hline Tjønna et al. (2009) & Insulin $\left(\mathrm{pmol} \cdot \mathrm{1}^{-1}\right)$ & $-54.30 *$ & $-33.00 *$ \\
\hline
\end{tabular}

Significantly different from baseline $\left(* \mathrm{p}<0.05,{ }^{*} \mathrm{p}<0.01\right),{ }^{\mathrm{a}}$ values adjusted for sex, baseline value and matur calculated due to no reported post-intervention means, HIIT high-intensity interval training group, CI confide lipoprotein cholesterol, LDL low-density lipoprotein cholesterol 


\section{Discussion}

The aim of the current review was to synthesise previous literature that examined HIIT in children and adolescents and establish its potential effect on body composition, cardiometabolic and cardiovascular health. In addition, this review aimed to identify an optimal HIIT protocol with regard to session structure, intensity, frequency and duration. In accord with this aim, 13 studies were evaluated providing evidence suggesting that HIIT can significantly improve certain health parameters in children and adolescents. However, evidence supporting the overall effectiveness of HIIT as a means of eliciting improvements to all the specified health outcomes remains unclear. Some guidelines for a HIIT protocol were established, though recommendations for certain protocol details remain unidentified.

Advancing previous reviews (Costigan, Eather, Plotnikoff, Taaffe, \& Lubans, 2015; Logan et al., 2014), the findings of the current review suggest that pubertal children may achieve a greater benefit as a result of HIIT when compared to pre-pubertal children, a topic that has been widely debated (McNarry, Mackintosh, \& Stoedefalke, 2014; McNarry, Welsman, \& Jones, 2011; Mirwald, 1981; Ostojic, Stojanovic, Jukic, Pasalic, \& Jourkesh, 2009). However, it is pertinent to note that this may be a consequence of several methodological factors that restrict the interpretation of previous studies. Firstly, the duration of the HIIT interventions examining pubertal participants tended to be longer than those in pre-pubertal children. Given the present findings suggesting that a minimum of 7 weeks is required for significant adaptations to be manifest, this shorter intervention duration may lead to erroneous conclusions regarding the efficacy of HIIT in this population. Furthermore, differences in the participant characteristics between pre-pubertal and pubertal studies with regard to baseline body mass or body composition and health status may confound interpretation of inter-study differences and their attribution to maturity per se. Specifically, the majority of studies in pre-pubertal children utilised those of a normal weight compared to the inclusion of overweight or obese participants in pubertal studies, which may predispose these latter studies to demonstrating greater health benefits, irrespective of their biological age. Moreover, both Tjønna et al. (2009) and Racil et al. (2016) did not report maturation stages, subsequently casting ambiguity over the cohort's true maturational stage. Despite this, when focusing on the additional study involving pubertal children (K. L. Weston et al., 2016), it generally elicited greater 
improvements in outcome measures when compared to the study involving prepubertal children (Rosenkranz et al., 2012). Moreover, only two of the studies involving pubertal children that demonstrated positive significant results (Racil et al., 2016; Tjønna et al., 2009) considered dietary intake; a failure to account for changes in dietary intake, which is strongly associated with cardiometabolic health (Funtikova, Navarro, Bawaked, Fíto, \& Schröder, 2015) and obesity (Gillis, Kennedy, Gillis, \& Bar-Or, 2002) in children and adolescents, confounds the interpretation of the results and their attribution to the exercise per se. Finally, in addition to the aforementioned methodological limitations, the interpretation of the overall findings of this study may also be limited by mixed risk of bias between the significant studies.

An additional factor that may contribute to the collective ambiguity regarding the overall effectiveness of HIIT is the "compensation effect". Specifically, a recent study has suggested that school children appear to compensate for increased physical activity levels, with a reduction in physical activity undertaken the following day (Ridgers et al., 2014). Additionally, the "activitystat" hypothesis suggests that increased levels of physical activity during one part of the day may result in a compensatory decrease in physical activity in another part (Rowland, 1998). Therefore, there is scope to suggest that prescribing exercise that reflects the characteristics of children's comparatively elevated levels of habitual play (Brodersen, Steptoe, Boniface, \& Wardle, 2007) may result in a decrease in habitual physical activity levels that day or the subsequent day. This therefore highlights the need to measure habitual physical activity, as was the case for only four of the studies included within this review (Boddy et al., 2010; Rosenkranz et al., 2012; Tjønna et al., 2009; K. L. Weston et al., 2016), alongside the previously outlined study outcomes. Regarding the effect of HIIT between sexes, no conclusions could be drawn as none of the studies included in this review provided a breakdown of between sex differences. This could have implications for future research, given the possibility that effects of exercise interventions on body size (Lazaar et al., 2007; Martínez-Vizcaíno et al., 2014), cardiorespiratory (Obert et al., 2003) and cardiometabolic health (Martínez-Vizcaíno et al., 2014) may be sexdependent in children. Therefore, future studies should endeavour to report a more expansive breakdown of results, thus providing clarification as to possible sex and maturational differences associated with HIIT. 
Whilst the studies within this review have advanced our understanding regarding the influence of HIIT in children under laboratory-based conditions, the relatively small sample sizes and intervention delivery methods highlight potential issues regarding larger scale implementation of HIIT. Schools have frequently been used as a foundation in the implementation of physical activity interventions (Christian et al., 2016; Mackintosh et al., 2016) due to their access to a greater population of children, who spend $40 \%$ of their waking hours there (Fox et al., 2004), and are widely accepted as one of the most effective locations to promote physical activity and health. Furthermore, previous studies have demonstrated how short-term HIIT interventions have been successfully embedded within the school timetable (Boddy et al., 2010; Costigan, Eather, Plotnikoff, Taaffe, Pollock, et al., 2015). It has been suggested that HIIT can allow for greater class control compared to conventional physical education lessons, and can be adapted to include specific movements related to different sports (Ratel et al., 2004). Therefore, given the promising findings regarding the effectiveness of HIIT, future research may wish to consider how long-term HIIT interventions could be incorporated within the school environment. A key consideration in the development of future interventions, and participants' engagement in and adherence to the devised program, is a participant's perceived enjoyment. Whilst HIIT has been suggested to be a preferable exercise modality to more conventional, aerobic exercise (Logan et al., 2014), further research is required, with only one study in the present review considering this aspect of intervention development and implementation (Lambrick et al., 2016). Finally, the sustained postintervention efficacy of HIIT interventions that reported significant improvements to body composition, cardiometabolic and cardiovascular health in children and adolescents remains indeterminate owing to a predominant scarcity of studies reporting a post-intervention follow-up. Therefore, future studies should incorporate a follow-up period within their study design to assess the long-term post-intervention sustainability of positive HIIT elicited benefits.

\section{Conclusion}

High-intensity interval training is a time-effective method of improving cardiovascular disease biomarker health in children and adolescents. However, evidence supporting its effectiveness in additional health measures remains equivocal. This review suggests that running-based sessions, at an intensity of $>90 \%$ maximum HR/100-130\% 
maximal aerobic velocity, 2-3 times a week and with a minimum intervention duration lasting $>7$ weeks, elicit improvements in health markers; however, these findings are limited by the mixed risk of bias between the significant studies. Further recommendations as to exercise duration and rest intervals remain ambiguous due to the paucity and methodological limitations of studies presently available. 


\section{Thesis study map}

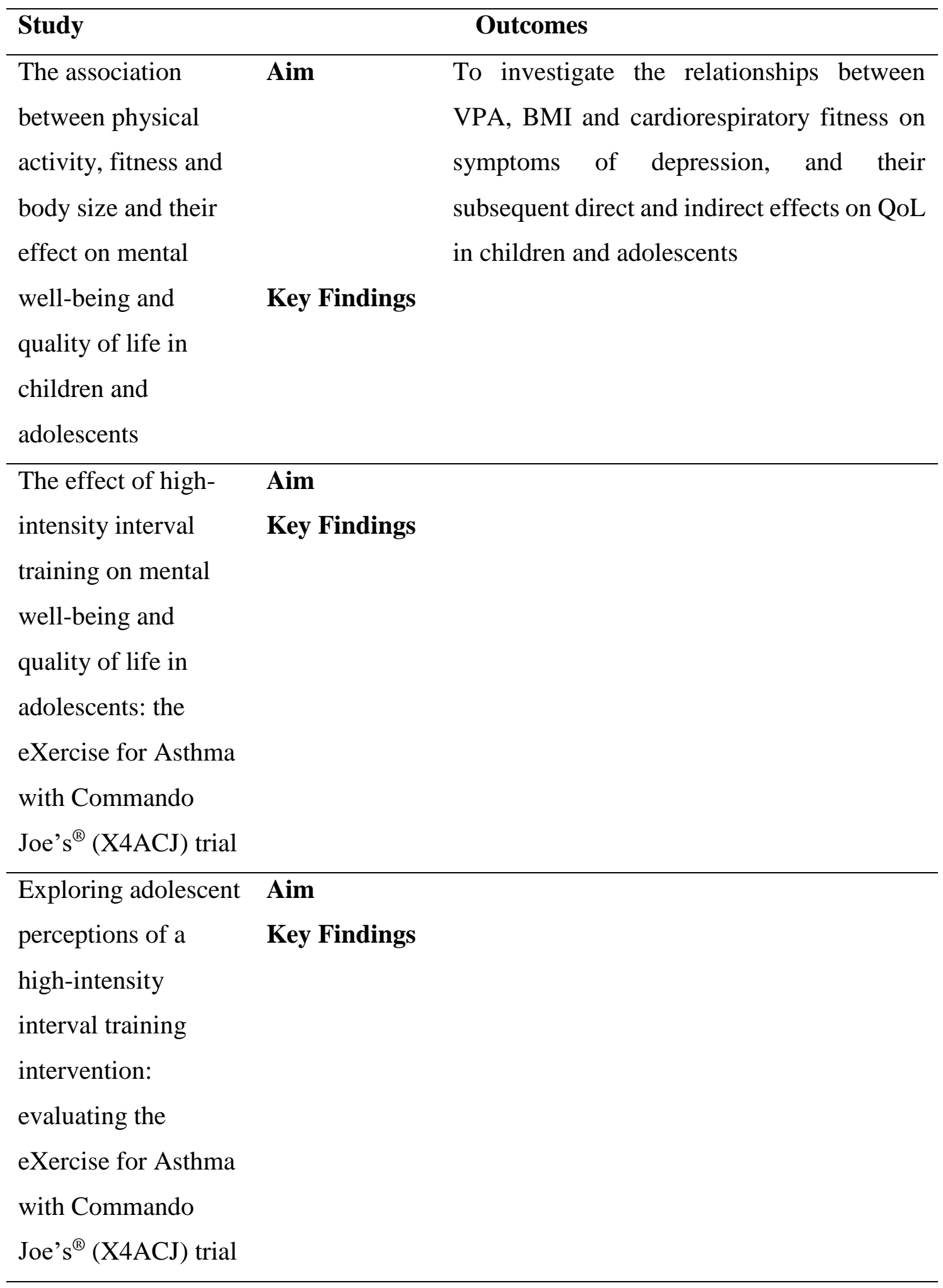




\section{Chapter 4 - The association between physical activity, fitness and body size and their effect on mental well-being and quality of life in children and adolescents (Study 1)}

The following chapter has been published as an original article in a peer-reviewed journal (Eddolls, W. T. B., McNarry, M. A., Lester, L., Winn, C. O. N., Stratton, G., \& Mackintosh, K. A. (2018). The association between physical activity, fitness and body mass index on mental well-being and quality of life in adolescents. Quality of Life Research). With the exception of some minor amendments, it has been presented in its published form. The fully published version of this study can be found in Appendix B (p. 190-197).

\section{Introduction}

Quality of life (QoL), a subset of health defined by the World Health Organisation to include the physical, mental and social well-being of a person (World Health Organization, 2006), is widely recognised as a fundamental element in the evaluation of population health (Helseth \& Misvær, 2010) and should be considered the underlying target for health interventions (Ravens-Sieberer, Herdman, et al., 2014). Nonetheless, despite its widely accepted importance, recent findings have suggested that self-reported QoL has significantly declined in adolescents over the last decade (Cui \& Zack, 2013), highlighting the need for interventions that specifically target QoL in this population. However, due to the subjective nature of QoL, it cannot be directly enhanced (Zack, 2013). Therefore, the influence of health-related parameters warrants further attention as potential mediators through which to enhance QoL.

Previous research has shown that interventions that modify certain health behaviours associated with QoL, such as physical health and mental well-being, can indirectly elicit improvements in QoL. Cardiorespiratory fitness and body mass index (BMI), which are important indicators of health (Ortega et al., 2008; Ruiz et al., 2009) that predict one another (Monyeki et al., 2012), also predict QoL; those with a higher cardiorespiratory fitness experience a better QoL (J. R. Andersen et al., 2017; Morales et al., 2013; Sloan et al., 2009), and overweight or obese individuals typically experience a poorer QoL (Swallen et al., 2005; J. Williams et al., 2005). Moreover, mental well-being and low physical activity levels, further prominent indicators of health (Warburton, Nicol, \& Bredin, 2006; World Health Organization, 2006), have 
also been shown to influence QoL, with those suffering from mental health issues, or not meeting physical activity guidelines of 60 minutes of moderate-to-vigorous physical activity per day, at greater risk of a poor QoL (Sharpe et al., 2016; Wafa et al., 2016). However, despite these predictors being identified, the interaction between them and the influence of mental health on QoL remains to be elucidated. Indeed, little research has explored the potential indirect relationships between health behaviours and their mediating and interactive influence on QoL. For example, when compared to moderate physical activity (MPA), vigorous physical activity (VPA) has been associated with fewer symptoms of depression (Gerber et al., 2014), the most common form of mental illness in the UK (National Collaborating Centre for Mental Health, 2011) and has also been shown to predict cardiorespiratory fitness and adiposity (Parikh \& Stratton, 2011) more time-efficiently (I.-M. Lee \& Paffenbarger, 2000; Yu, Yarnell, Sweetnam, \& Murray, 2003). Thus, VPA levels have the potential to indirectly, yet substantially, affect QoL through symptoms of depression, adiposity and cardiovascular fitness.

In addition to providing a more comprehensive oversight of the mediating factors affecting QoL, establishing a link between body composition, cardiorespiratory fitness, symptoms of depression, QoL and VPA, specifically, may also help to inform future research. Indeed, research has suggested that individuals may be able to achieve the benefits accrued from achieving physical activity guidelines more time-efficiently by substituting moderate physical activity with VPA (I.-M. Lee \& Paffenbarger, 2000; Yu, Yarnell, Sweetnam, \& Murray, 2003). Furthermore, Parfitt et al. (2009) found that, compared to light and moderate physical activity, VPA was associated with a positive psychological profile. Moreover, given the association between VPA and highintensity interval training (HIIT; Costigan et al., 2017), determining the relationship between VPA, physical health, mental well-being and QoL may also substantiate previously outlined physiological HIIT protocol guidelines (Eddolls, McNarry, Stratton, Winn, \& Mackintosh, 2017) within a psychological and QoL setting.

Therefore, the purpose of the current study was to investigate the relationships between VPA, BMI and cardiorespiratory fitness on symptoms of depression, and their subsequent direct and indirect effects on QoL in children and adolescents using structural equation modelling (SEM). 


\section{Methods}

\section{Participants}

Five comprehensive schools located in South Wales (UK) were randomly stratified from fifteen who were initially invited to participate in a wider randomised control trial (the X4A trial: eXercise for Asthma with Commando Joe's) and included within this study. Based on the most recent Estyn reports and the Welsh Index of Multiple Deprivation, the participating schools ranged in size from 625-1,119 pupils, with deprivation ranging between the second and fifth quintiles. Given that participating schools were unwilling to allow their year 11 pupils to participate due to the academic pressures of examinations, only years 7-10 were invited to participate and provided information sheets. Following headteacher and parental consent and child/adolescent assent (Appendix C-K; p. 198-219), five hundred and seventy-six children and adolescents (314 boys, $12.5 \pm 1.1$ years) participated. Within the intervention school and largest control school, from an estimated 1,500 pupils, 28\% returned parental consent and child assent forms. For the 3 additional schools, however, only specific physical education lessons could be targeted with total number of children eligible not known. As such, information as to the participation rate for these schools is not available. Measures were taken over approximately 6 weeks at the beginning of the academic year (September-October). Participants that were included within Studies 2 and 3 were also included within this study. Approval to conduct this research was granted by the institutional ethical advisory committee (ethics number: PG/2014/29) and performed in accordance with the Declaration of Helsinki.

\section{Measures}

\section{Anthropometric and maturity assessment}

Stature and body mass were measured, to the nearest $0.1 \mathrm{~cm}$ and $0.1 \mathrm{~kg}$, respectively, using a stadiometer (Seca 213, Seca Ltd, Birmingham, UK) and body mass scales (Seca 899, Seca Ltd, Birmingham, UK). From these measures, BMI was calculated. Given the ease of data collection and analysis in addition to the risk of multicollinearity with BMI by using peak height velocity (Mirwald, Baxter-Jones, Bailey, $\&$ Beunen, 2002), maturational status was assessed using self-reported indices of pubic hair, as described by Tanner (1962; Appendices L \& M; p. 220-221). Considered the 
gold-standard for measuring pubertal stage (Schmitz et al., 2004), self-reported Tanner stage pubertal assessment is considered sufficiently accurate for large epidemiological studies (Rasmussen et al., 2015).

\section{Physical activity levels}

Measures of physical activity are vast with a wide array of both objective and subjective tools available. Whilst subjective measures such as questionnaires have previously been utilised in large scale studies, these methods have several limitations (Shephard, 2003). Consequently, only objective measures, such as heart rate (HR) telemetry and accelerometery, were considered. Although Actihearts (CamNTech, Cambridge, UK) are perhaps considered the leading field instrument for measuring energy expenditure (Butte et al., 2010), their cost was prohibitive. As such the GT3X+ (ActiGraph, Pensacola, FL, USA) was used to measure and record the quantity, intensity and frequency of body movement. Although other accelerometer devices such as the GENEActiv (Activinsights, Kimbolton, UK) are available, the GT3X+ is widely considered as the leading valid and reliable objective measure of physical activity (Trost, 2007), which is relatively inexpensive compared to objectively assessed energy expenditure. Furthermore, given the abundance of accelerometers available to the study, using the GT3X+ was identified as a much more pragmatic approach. As such, participants were asked to wear the GT3X+ accelerometer, set at $100 \mathrm{~Hz}$, on their right mid-axilla line at the level of the iliac crest for seven full days, only removing it if they undertook contact or water-based activities. Wear-time diaries were used to $\log$ why the accelerometers were taken off and for how long. Data was analysed using KineSoft (version 3.3.67; KineSoft, Saskatchewan, Canada) employing 1 second epochs with sustained periods of at least 20 minutes at zero counts considered non-wear-time (Catellier et al., 2005). A minimum daily wear-time of 10 hours for 3 days, including 1 weekend day, was selected in order to provide a more accurate overview of participants habitual physical activity levels (Rich et al., 2013). Of the 576 participants who received an accelerometer, 323 (56\%) met this criteria. Evenson, Catellier, Gill, Ondrak and McMurray (2008) cut-points, shown to be valid and reliable determinants of activity intensity in children and adolescents (Trost, Loprinzi, Moore, \& Pfeiffer, 2011), were used to calculate time spent in vigorous physical activity (>4012 counts per minute). 


\section{Depression}

Although there are other questionnaires available that perhaps have greater validity, for example the Hamilton Rating Scale for Depression (HAM-D; Hamilton, 1960) and the Beck Depression Inventory (BDI; Beck et al., 1961), these scales were not pragmatic nor feasible. Indeed, the HAM-D requires a trained clinician to administer the test on an individual basis and the BDI is copyrighted and requires a fee for each copy used. As such, symptoms of depression were measured using the Center for Epidemiologic Studies Depression Scale for Children (CES-DC) questionnaire (Weissman et al., 1980; Appendix N; p. 222). Validated for use within the child and adolescent population (Binagwaho, Stulac, \& Beardslee, 2012; Fendrich, Weissman, \& Warner, 1990; Stockings et al., 2015), the CES-DC is a 20-item scale with overall scores ranging from 0-60 with higher scores representing a greater level of depressive symptoms. In order to assess the reliability or internal consistency of the test items, Cronbach's Alpha (Cronbach, 1951) was used. This method correlates each scale item with the total score for each observation, then compares it to the variance for all individual item scores. Scores can range between 0-1 with an $\alpha>0.7$ often deemed the minimum acceptable threshold. Internal reliability for the CES-DC, was deemed good $(\alpha=0.911)$.

\section{Quality of life}

Perceived QoL was assessed using the Pediatric Quality of Life Inventory (PedsQL) Teenager Report, Version 4.0 (Varni et al., 1999; Appendix O; p. 223), a widely validated 23-item scale designed for use with participants aged between 13-18 years (Varni et al., 2006, 2003, 2001). Within the construct of the 23-item questionnaire, the measure is broken down into four scales: physical $(n=8)$, emotional $(n=5)$, social ( $n$ $=5)$ and school functioning $(\mathrm{n}=5)$. From these scales, physical $(\mathrm{n}=8)$ and psychological $(n=15)$ summary scores were calculated. Scores ranged between 0-100, with higher scores indicative of a better QoL. In line with Chapter 2, whilst the 57item KIDSCREEN (Ravens-Sieberer et al., 2005) has shown comparative validity (Janssens et al., 2008), given the fewer number of items and smaller estimated completion time, the PedsQL was deemed the most pragmatic choice. Internal reliabilities, for physical QoL $(\alpha=0.759)$ and psychological QoL $(\alpha=0.875)$ deemed acceptable (Cronbach, 1951). 


\section{Cardiorespiratory fitness}

Cardiorespiratory fitness was estimated using the multi-stage fitness test (MSFT), a previously validated field measure in children (Boreham, Paliczka, \& Nichols, 1990; Mayorga-Vega, Aguilar-Soto, \& Viciana, 2015). The MSFT was conducted over a 20metre space, in a hard-floor indoor sports hall, with the total number of shuttles completed used as a score marker.

\section{Statistical analysis}

Analysis was guided by Ferrans, Zerwic, Wilbur and Larson's (2005) updated Wilson and Cleary (1995) conceptual model, which hypothesised that measured health outcomes can predict QoL. Data were analysed using IBM SPSS Statistics for Windows, Version 22.0 (IBM Corp, Armonk, NY, 2013) and IBM SPSS AMOS for Windows, Version 22.0 (IBM Corp, Armonk, NY, 2013). Missing data ( $\mathrm{n}=903$, 17.4\%) were imputed using an expectation-maximisation (EM) algorithm. For BMI, cardiorespiratory fitness, anxiety, depression and QoL, the reasons for missing data was as a result of participants being absent from school or unavailable when data collection took place. For physical activity, there was poor wear-time compliance and therefore the physical activity data of all participants not meeting the wear-time criteria were excluded. Structural equation modelling was used to evaluate the difference in linear structural associations between variables. Direct effects were estimated using direct path coefficients between two measured variables and indirect associations were estimated as a product of two direct effects between three measured variables. This second-generation multivariate technique is used to analyse structural relations using a combination of factor analysis and multiple regression. In contrast to first-generation statistical techniques such as multiple regression, using structural equation modelling allows for multiple dependent variables and can simultaneously evaluate model construct relationships. Given the potential confounding effects in children and adolescents (Baxter-Jones, Eisenmann, \& Sherar, 2005), sex and maturation were accounted for within the model. All data are presented as mean $\pm \mathrm{SD}$, with a statistically significant criterion set at $p<0.05$. For analysis, unless otherwise stated, data were standardised $(\beta)$. 


\section{Results}

Dichotomised by sex, the means and SD of maturation, BMI, cardiorespiratory fitness, depression, physical and psychological QoL and VPA are presented in Table 4.1.

Table 4.1 Means and SD among of the measured variables of the study participants, dichotomised by sex.

\begin{tabular}{lcc}
\hline & $\begin{array}{c}\text { Boys } \\
(\mathbf{n}=\mathbf{3 1 4})\end{array}$ & $\begin{array}{c}\text { Girls } \\
(\mathbf{n}=\mathbf{2 6 2})\end{array}$ \\
\hline Maturation & $3.4 \pm 1.0$ & $3.0 \pm 1.0$ \\
BMI & $20.9 \pm 4.1$ & $20.7 \pm 3.7$ \\
MSFT & $46.9 \pm 23.1$ & $33.2 \pm 14.7$ \\
CES-DC & $14.3 \pm 10.0$ & $20.3 \pm 11.6$ \\
PedsQL (Physical) & $81.1 \pm 14.9$ & $77.1 \pm 14.8$ \\
PedsQL (Psychological) & $78.6 \pm 15.8$ & $73.3 \pm 17.0$ \\
VPA & $26 \pm 10$ & $22 \pm 7$ \\
\hline
\end{tabular}

SD standard deviation, MSFT multi-stage fitness test, BMI body mass index, CESDC Center for Epidemiologic Studies Depression Scale, PedsQL Pediatric Quality of Life Inventory, VPA vigorous physical activity

\section{Confirmatory factor analysis}

Inspection of the model fit indices $(\mathrm{NFI}=0.981, \mathrm{TLI}=0.982, \mathrm{CFI}=0.991$, RMSEA $<0.05)$ suggested that the model was acceptable. Standardised parameter estimates for the measurement model are provided in Figure 4.1.

\section{Structural equation model}

Standardised and unstandardised regression weights with standard errors between variables included within the model are provided within Table 4.2.

\section{Vigorous physical activity}

The model (Figure 4.1) revealed that VPA was directly related to BMI $(\beta=-0.293, p$ $<0.001)$ and cardiorespiratory fitness $(\beta=0.242, p<0.05)$, and indirectly related to depressive symptoms scores $(\beta=-0.034, p<0.001)$ and physical QoL $(\beta=-0.038, p$ $<0.001)$, mediated by BMI and cardiorespiratory fitness, respectively.

\section{Body mass index}

Body mass index was directly related to cardiorespiratory fitness $(\beta=-0.331, p<$ $0.001)$ and depressive symptoms scores $(\beta=0.117, p<0.05)$. Indirect relationships 
were found between BMI and physical QoL, mediated by cardiorespiratory fitness ( $\beta$ $=0.053, p<0.001)$ and symptoms of depression $(\beta=-0.061, p<0.001)$. Additionally, an indirect relationship was found between BMI and psychological QoL $(\beta=-0.092$, $p<0.001)$, mediated by symptoms of depression.

\section{Cardiorespiratory fitness}

Cardiorespiratory fitness had direct relationship with physical QoL $(\beta=0.159, p<$ $0.001)$

\section{Depression}

There was a direct relationship between symptoms of depression and psychological QoL $(\beta=-0.788, p<0.01)$ and physical QoL $(\beta=-0.519, p<0.001)$.

\section{Maturation and sex}

Maturation directly affected BMI $(\beta=0.147, p<0.001)$ and cardiorespiratory fitness scores $(\beta=0.255, p<0.001)$, whilst sex was found to directly affect VPA $(\beta=-0.179$, $p<0.01)$, cardiorespiratory fitness $(\beta=-0.237, p<0.001)$ and depressive symptoms $(\beta=0.270, p<0.001)$.

Table 4.2 Standardised and unstandardised regression weights with standard errors

\begin{tabular}{lccc}
\hline Paths & $\boldsymbol{\beta}$ & B & SE \\
\hline Sex $\rightarrow$ VPA & -0.18 & -3.34 & 0.76 \\
Maturation $\rightarrow$ BMI & 0.15 & 0.55 & 0.15 \\
VPA $\rightarrow$ BMI & -0.29 & -0.13 & 0.02 \\
Maturation $\rightarrow$ MSFT & 0.26 & 0.53 & 0.07 \\
BMI $\rightarrow$ MSFT & -0.33 & -0.18 & 0.02 \\
BMI $\rightarrow$ CESDC & 0.12 & 0.33 & 0.11 \\
VPA $\rightarrow$ MSFT & 0.24 & 0.06 & 0.01 \\
Sex $\rightarrow$ CESDC & 0.27 & 6.04 & 0.89 \\
Sex $\rightarrow$ MSFT & -0.24 & -1.04 & 0.15 \\
CESDC $\rightarrow$ PedsQL (Psychological) & -0.79 & -1.17 & 0.04 \\
MSFT $\rightarrow$ PedsQL (Physical) & 0.13 & 0.89 & 0.20 \\
CESDC $\rightarrow$ PedsQL (Physical) & -0.53 & -0.70 & 0.05 \\
\hline
\end{tabular}

$\beta$ standardised regression weight, B unstandardised regression weight, SE standard error, MSFT multi-stage fitness test, BMI body mass index, CES-DC Center for Epidemiologic Studies Depression Scale, PedsQL Pediatric Quality of Life Inventory, VPA vigorous physical activity 


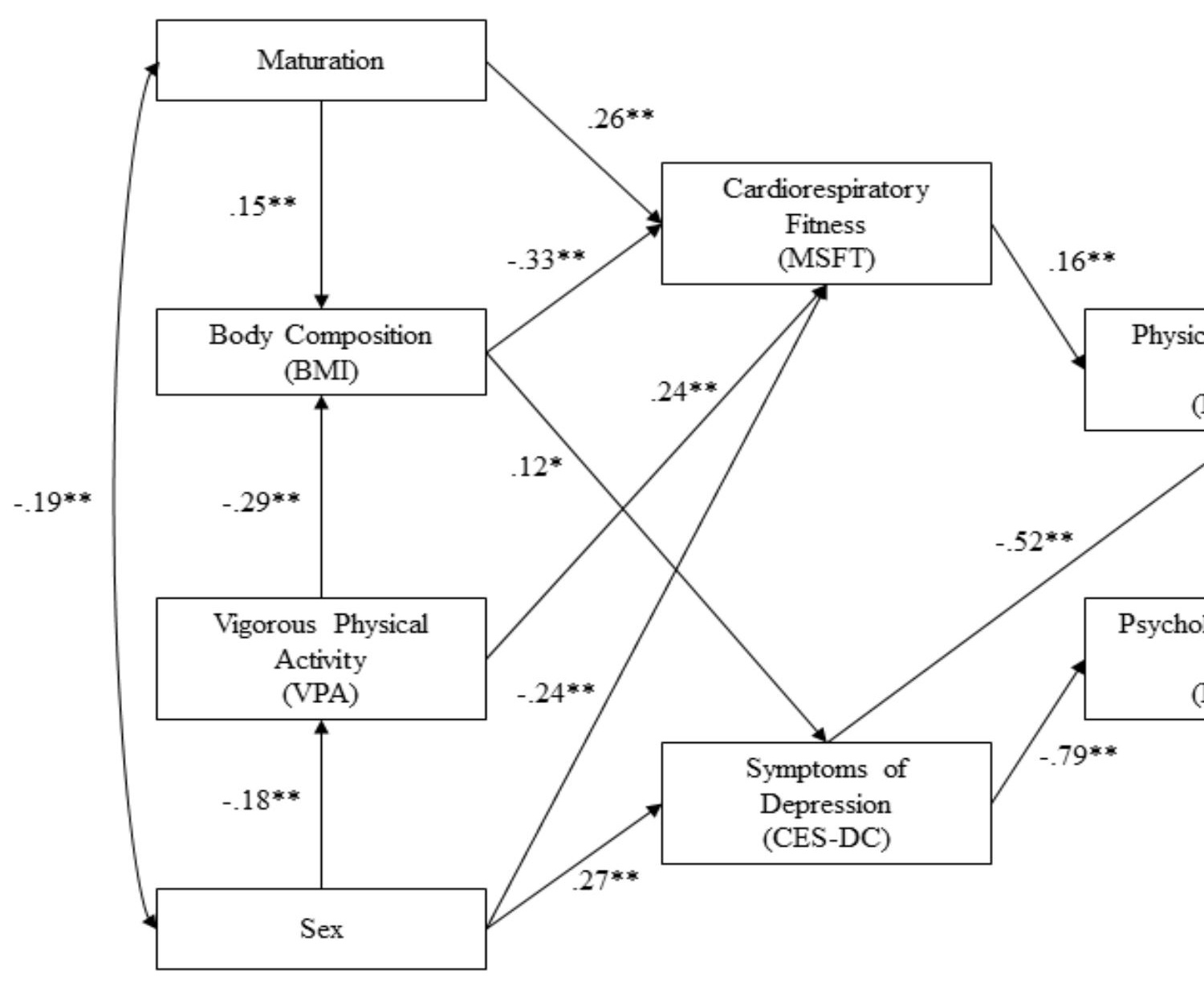

Figure 4.1 Structural equation model (SEM) of physical activity levels, physiological health and their effect life in children and adolescents with standardised beta coefficients $\left(* \mathrm{p}<0.05,{ }^{* *} \mathrm{p}<0.01\right)$ 


\section{Discussion}

This study investigated the relationship and mediatory effects between physical activity levels, BMI, cardiorespiratory fitness and symptoms of depression, and their subsequent effect on physical and psychological QoL. Models suggested that VPA, cardiorespiratory fitness and BMI were associated, both directly and indirectly, with mental well-being and physical and psychological QoL. It could therefore be postulated that enhancing cardiorespiratory fitness and BMI through increasing VPA may be beneficial to both mental well-being and QoL in children and adolescents.

The current results show that, in accord with previous literature (Luppino et al., 2010; Roberts, Deleger, Strawbridge, \& Kaplan, 2003), a healthier BMI is associated with fewer symptoms of depression. It is possible that this association, at least in part, may be due to psychosocial factors associated with BMI. For example, stigmatisation towards those that have a higher BMI may cause lower self-esteem, induce negative self-images and lead to perceptions of social isolation, thus eliciting depressive symptoms (C. E. Ross, 1994). A review by Markowitz et al. (2008) also proposes that the association between obesity and depression is related to concerns regarding selfperceived health and appearance, whereby those with higher BMI experience, or are more vulnerable to, depression. Physiologically, overweight and obesity may lead to hypothalamic-pituitary-adrenal (HPA) axis dysregulation (Pasquali \& Vicennati, 2000) and the onset of comorbidities, such as insulin resistance and consequent type II diabetes (Bastard et al., 2006), both of which are associated with alterations to the brain and increase the likelihood of depressive symptoms (Ajilore et al., 2007; Belanoff, Kalehzan, Sund, Fleming Ficek, \& Schatzberg, 2001; Holsboer, 2000).

Congruent with previous research (Sharpe et al., 2016), findings from the present study suggest that lower levels of depressive symptoms were associated with higher levels of psychological and physical QoL, highlighting the link between mental well-being and QoL. In addition to the direct associations with depressive symptoms, the model also proposes that a healthier BMI is indirectly associated with a greater psychological and physical QoL, mediated by levels of depressive symptoms and cardiorespiratory fitness, respectively. As outlined in previous literature (Kearns, Ara, Young, \& Relton, 2013; Mclaughlin \& Hinyard, 2014; Sach et al., 2007), these findings, therefore, identify BMI as a key influential factor that can significantly affect an individual's 
QoL. Indeed, interventions designed to improve QoL via a reduction in BMI have reported positive results (Rippe et al., 1998; Rothberg et al., 2015).

Beyond depressive symptoms and BMI, participants with a higher cardiorespiratory fitness also experienced a better physical QoL (J. R. Andersen et al., 2017). Given the link between cardiorespiratory fitness and BMI observed in this study and elsewhere (Stratton et al., 2007), the association between cardiorespiratory fitness and physical QoL (Lowery et al., 2005) may also be related to a negative self-image and self-esteem (C. E. Ross, 1994), or body-image (Nayir, Uskun, Yürekli, Çelick, \& Okyay, 2016), components that are encompassed within the physical QoL measurement. In addition to psychosocial issues, given that disease impairs QoL (Häkkinen et al., 2010), it could be postulated that the effect of cardiorespiratory fitness on QoL may be related to its association with an increased risk of physical morbidities (D. Lee, Artero, Sui, \& Blair, 2010). However, the physiological mechanisms explaining the connection between cardiorespiratory fitness and QoL are not entirely understood (Clennin et al., 2015) and therefore warrant further research. Nevertheless, given the relationship between cardiorespiratory fitness and exercise (Garber et al., 2011), it could be postulated that exercise may be an effective means of improving QoL. More specifically, given that recent research has suggested that HIIT is a more potent and time-efficient method of inducing improvements to cardiorespiratory fitness (Tjønna et al., 2009; K. L. Weston et al., 2016), it could be proposed that HIIT could significantly promote QoL.

In support of HIIT as an effective and time-efficient form of exercise, given its association with increased VPA (Costigan et al., 2017), a further interesting finding was that, whilst higher levels of VPA were directly associated with lower BMI and increased cardiorespiratory fitness (Drenowatz et al., 2016; Kenchaiah et al., 2009), higher levels of VPA were also indirectly associated with fewer symptoms of depression, mediated by BMI. It is postulated that, similar to the mechanisms proposed previously (Ajilore et al., 2007; Belanoff et al., 2001; Holsboer, 2000; Markowitz et al., 2008; C. E. Ross, 1994), the negative correlation between VPA and BMI may be associated with improvements in symptoms of depression, due to greater regulation of the HPA axis and insulin control, as well as improved self-perceptions in both appearance and health. Consequently, increasing VPA may elicit improvements to not only a person's physical health, but also may improve mental well-being. Indeed, Fox (1999) found that healthy adults should consider vigorous exercise as a means of 
improving mental well-being. However, with the exception of Parfitt et al. (2009) and Gerber et al. (2014), who found VPA elicited mental health benefits beyond those engendered through low physical activity and moderate physical activity, there is limited research that focuses on the effects of VPA on mental well-being, especially in children and adolescents. Regardless of the limited research and lack of consensus (Costigan et al., 2016; Shepherd et al., 2015), the identification of VPA as a moderator of mental health in the current study, Gerber et al. (2014) and Parfitt et al. (2009) is promising. Indeed, it potentially presents a newfound advocacy for the potential of HIIT, to directly and indirectly improve symptoms of depression and both physical and psychological QoL, mediated through the well-documented physiological health benefits (Racil et al., 2016; Rosenkranz et al., 2012; Tjønna et al., 2009; K. L. Weston et al., 2016).

Congruent with previous research (Pate, Wang, Dowda, Farrell, \& O’Neill, 2006; Piccinelli \& Wilkinson, 1999), the present study demonstrated that sex had a significant relationship with both cardiorespiratory fitness and symptoms of depression; girls were more likely to experience poorer fitness and mental well-being. These differences may, in part, be related to environmental considerations, for example, sociocultural roles and psychological attributes (Piccinelli \& Wilkinson, 1999) or hormonal factors (Nolen-Hoeksema, 2001). However, it is also important to consider the importance of the divergence in physical development during puberty, such as changes to lean muscle and fat (Pate et al., 2006) relating to psychosocial factors associated with overweight or obesity (Markowitz et al., 2008; C. E. Ross, 1994), therefore, further highlighting the need for health interventions to primarily focus on improving BMI in order to enhance overall health.

This is the first study to use SEM to determine both direct and indirect associations between physiological health, physical activity levels, mental well-being and QoL. Furthermore, the present study accounted for maturational status and sex, both of which are often overlooked in child and adolescent studies, as well as using robust, previously validated, measures of physical activity (Mattocks et al., 2008; MayorgaVega et al., 2015; Varni et al., 2006). Nonetheless, whilst this study is associated with numerous strengths, there are inevitably some limitations. It is anticipated that the omission of socio-economic status (SES) within the model, given its well-established relationship with the measured variables (Houben-van Herten, Bai, Hafkamp, 
Landgraf, \& Raat, 2015; Olson, Lara, \& Frintner, 2004), may limit the interpretation of the findings. Despite its frequent utilisation within clinical research, the application of BMI is commonly subject to scepticism due to the measure's inability to differentiate between lean and fat body mass (Nevill, Stewart, Olds, \& Holder, 2006). Additional limitations include the self-reporting of mental well-being and the associated disadvantages, such as social desirability (Paulhus \& Vazire, 2005). However, all of the questionnaires used were previously validated (Binagwaho et al., 2012; Fendrich et al., 1990; Stockings et al., 2015; Varni et al., 2006, 2003, 2001) and demonstrated acceptable reliability. Finally, primarily due to participants failing to meet accelerometer wear-time, expectation-maximisation was utilised to impute missing data and, whilst it has previously been reported as a valid method (Catellier et al., 2005; Nelwamondo, Mohamed, \& Marwala, 2007), it is important to acknowledge the influence the absence of a complete data set may have had on the findings within this study.

\section{Conclusion}

In conclusion, the present study found that VPA, cardiorespiratory fitness and BMI were associated, both directly and indirectly, with mental well-being and physical and psychological QoL. As such, it could be suggested that improving cardiorespiratory fitness and BMI by increasing VPA may be beneficial to both mental well-being and QoL in children and adolescents. Specifically, given the paucity of information and its relationship with VPA and cardiorespiratory fitness, future research should explore the effects of HIIT as a means to improve mental well-being and QoL. Furthermore, these findings suggest that, to elicit improvements to QoL, exercise or physical activity interventions should remain multi-faceted, simultaneously focusing on cardiorespiratory fitness, BMI and symptoms of depression. Consequently, the next chapter will assess the effects of a HIIT intervention on body mass index (BMI), cardiorespiratory fitness, physical activity levels, depression, anxiety and QoL in adolescents. 


\section{Thesis study map}

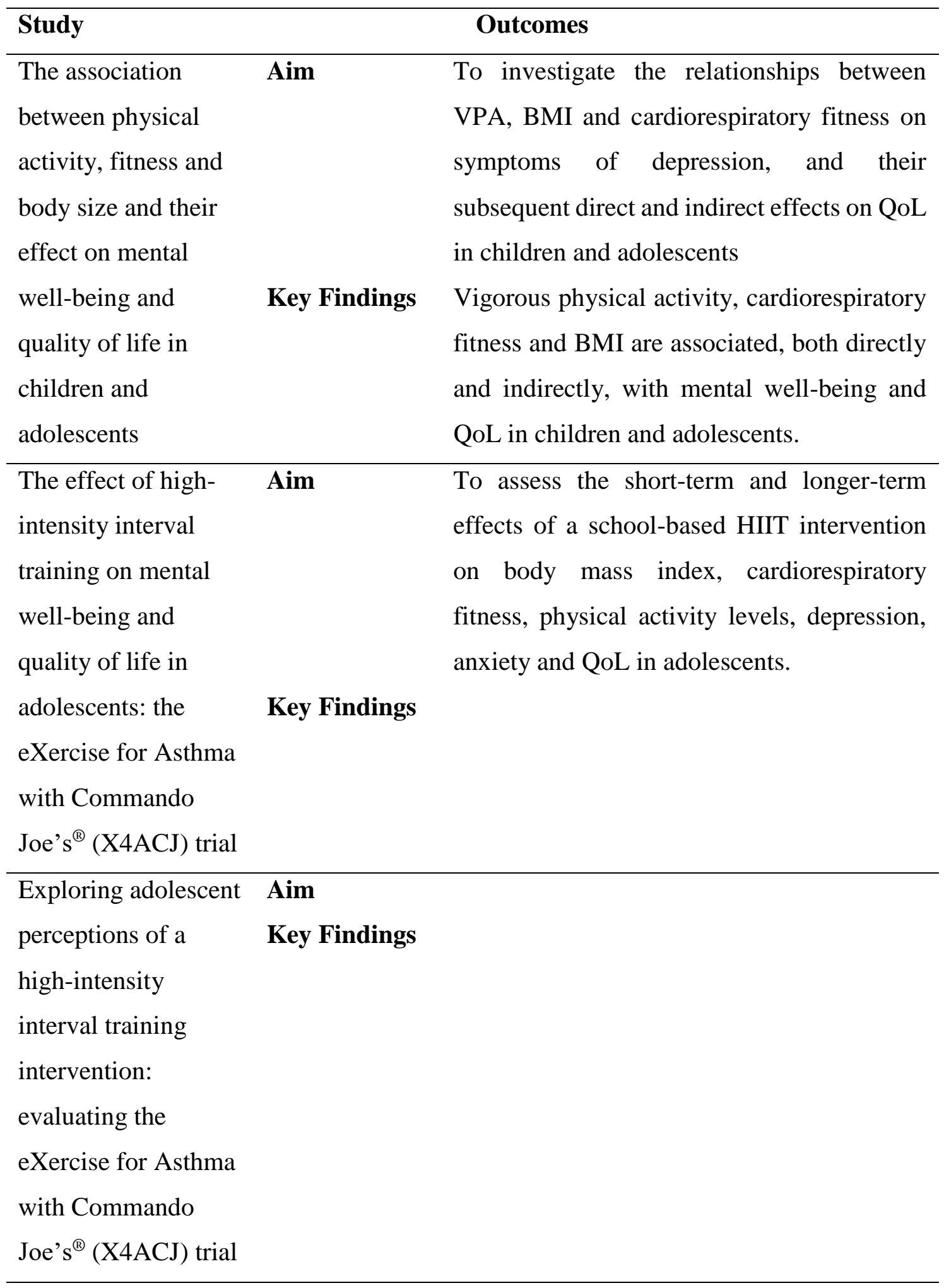


Chapter 5 - The effect of high-intensity interval training on mental well-being and quality of life in adolescents: the eXercise for Asthma with Commando Joe's ${ }^{\circledR}$ (X4ACJ) trial (Study 2)

\section{Introduction}

According to the World Health Organisation, 20\% of adolescents will experience a mental health problem (World Health Organization, 2003). Indeed, adolescents reporting recurrent sensations of anxiety or depression doubled between the mid 1980's and mid 2000's (Collishaw et al., 2010), with one-in-ten 5-16 years olds in the UK clinically diagnosed with a mental health disorder (Green et al., 2004). Furthermore, such mental illnesses have been associated with poorer quality of life (QoL; Sharpe et al., 2016), a fundamental element in the evaluation of adolescent health (Helseth \& Misvær, 2010), which has also declined during the last decade (Cui \& Zack, 2013). However, traditional methods for treating mental health issues, such as psychotherapy and pharmacological treatments, are expensive (McCrone et al., 2008), time-consuming (Gladstone et al., 2011) with doubts remaining over their effectiveness. Thus, preventing depression and anxiety themselves, rather than treating the symptoms, should be a priority, not least given the greater long-term effectiveness associated with improving depression and anxiety on QoL (Fisak et al., 2011).

Few effective mental health prevention strategies have been established (McCrone et al., 2008). Exercise, the structured form of physical activity, has been recognised to be associated with improved mental health and QoL (J. R. Andersen et al., 2017; Biddle \& Asare, 2011; Wafa et al., 2016), and has been recommended as a cost-effective approach for adolescents (Hagger et al., 2001; Larun et al., 2006). While the benefits of vigorous-intensity physical activity for mental well-being have been evidenced (Gerber et al., 2014; Parfitt et al., 2009), most exercise interventions have traditionally focused on high-volume, moderate-intensity or resistance-based exercise (Lau et al., 2004; Rees \& Sabia, 2010). However, such exercise interventions do not accurately resemble the habitual play patterns of adolescents (Brodersen et al., 2007) and are often time-consuming, a frequently cited barrier to exercise (Donahue et al., 2006; Lovell et al., 2010; Reichert et al., 2007; Welch et al., 2009). High-intensity interval training (HIIT) may, therefore, represent a time-efficient and cost-effective tool in eliciting improvements to mental health and QoL, in addition to the well documented 
physiological benefits (Costigan, Eather, Plotnikoff, Taaffe, \& Lubans, 2015; Eddolls et al., 2017; Chapter 3; Logan et al., 2014).

Whilst the effects of HIIT are promising, little research is currently available regarding its influence on mental health and QoL, with the limited evidence available largely contradictory. Whilst Costigan et al. (2016) reported that HIIT did not elicit significant improvements in mental well-being or QoL, Shepherd et al. (2015) found that HIIT significantly enhanced psychological well-being, with benefits similar to those achieved in the moderate-intensity comparison group. These discrepancies may be related to the relatively short intervention duration ( 8 weeks) utilised by Costigan et al. (2016), which may have been insufficient for beneficial effects to be manifest. Specifically, although H. E. Brown et al. (2013) suggested that the largest effects to depression were manifest in interventions lasting less than 3 months, a meta-analysis suggested that greater benefits were manifest in those who trained for 16 weeks or longer compared to those who trained for 9 weeks or less (Petruzzello et al., 1991). Given these contradictory findings, elucidation of possible minimum and maximal intervention duration thresholds in relation to the effects of HIIT on mental health are required. Furthermore, although previous research has explored the effect of shortterm HIIT (Boddy et al., 2010), there is a stark paucity of research examining the longer-term effects of HIIT on both physical and mental health.

Therefore, the purpose of the present study was to assess the short-term and longerterm effects of a school-based HIIT intervention on body mass index (BMI), cardiorespiratory fitness, physical activity levels, depression, anxiety and QoL in adolescents.

\section{Methods}

\section{Participants}

Eighty-one adolescents (45 boys; $12.8 \pm 1.1$ years; $1.58 \pm 0.01 \mathrm{~m} ; 51.8 \pm 14.1 \mathrm{~kg}$ ) from two comprehensive schools involved within the eXercise for Asthma with Commando Joe's $^{\circledR}(\mathrm{X} 4 \mathrm{ACJ})$ project were included within this study. As part of two Doctoral and

one Masters by Research programmes, the X4ACJ project (616 adolescents; 334 boys) was a wider trial that investigated the effects of physical activity and exercise on physical health, mental well-being and QoL in both the general adolescent population 
and adolescent populations with asthma. In the context of this thesis, however, participants were not stratified according to condition, with the percentage of participants with asthma (15\%) similar to the Welsh national average.

Recruited via email, telephone and face-to-face meetings, five schools matched for free school meal status were selected via random stratification from fifteen schools. Subsequently, using cluster randomisation, one school was allocated as the intervention school, with the remaining four allocated as the control schools. Within the intervention school, following recruitment assemblies and the return of parental/guardian consent and participant assent, two-hundred and twenty-three participants (116 boys) were recruited. However, despite power calculations suggesting that 47 intervention participants were required (Rosner, 2011), only 27 participants $(23 \% ; 15$ boys) attended an adequate number of sessions $(>70 \%)$ to warrant inclusion within this study. To increase statistical power, for the control group following recruitment assemblies, two participants were matched to each intervention participant based on sex, maturation and baseline scores. A flow-chart outlining the school recruitment process, group allocation and intervention completion rate is included within Figure 5.1. Consistent with Chapter 1, a school was chosen as the setting for the intervention due to the ability to reach a large number of young people from diverse socio-economic backgrounds (Fox et al., 2004). Furthermore, interventions that can be implemented within a "real world" setting are recommended (De Bourdeaudhuij et al., 2011) as they are less costly (Warren et al., 2003), have a greater chance of being maintained and, although not in this study, can be integrated within the curriculum. Approval to conduct this research was granted by the institutional ethical advisory committee (ethics number: PG/2014/29) and performed in accordance with the Declaration of Helsinki. 


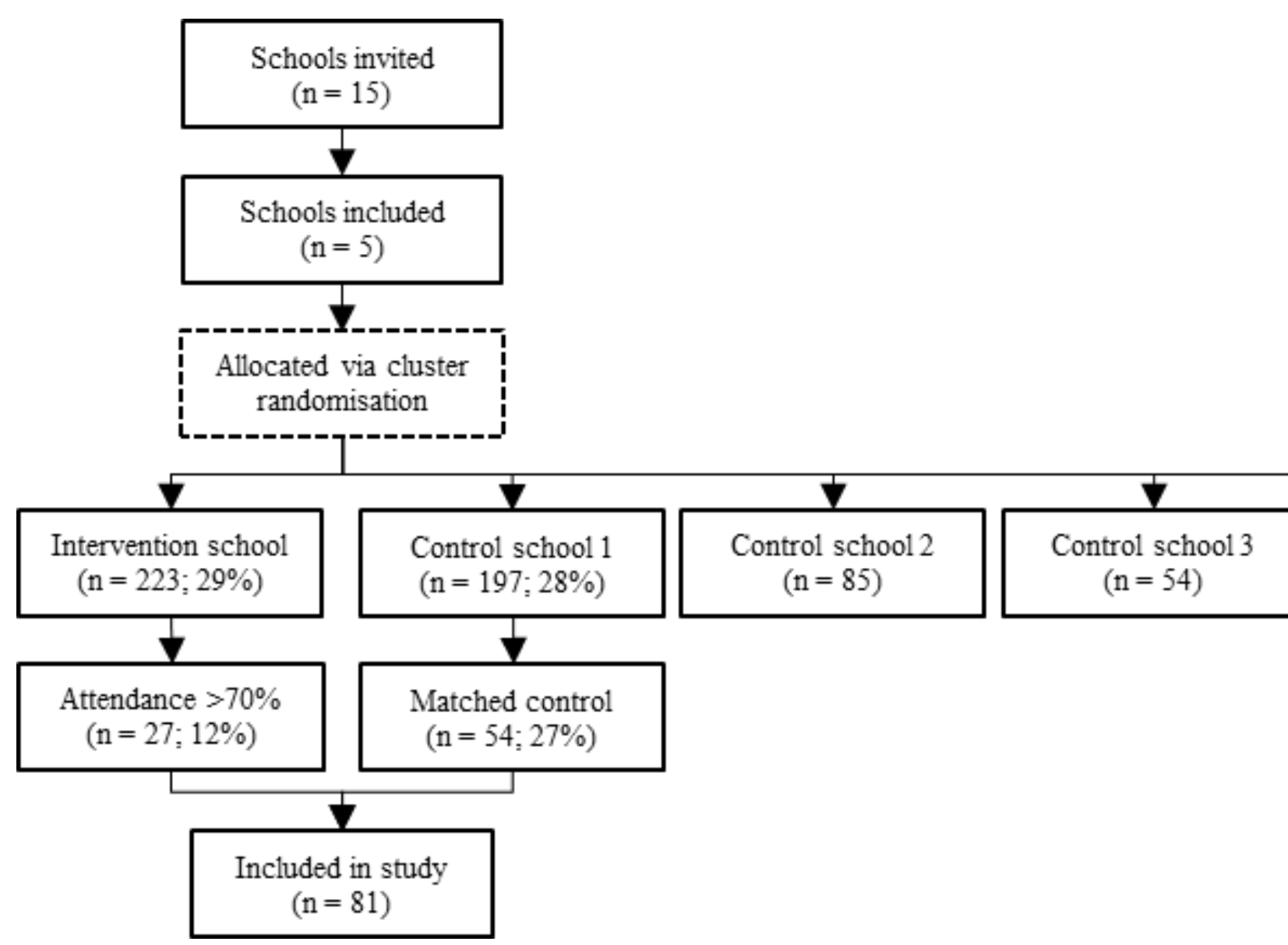

Figure 5.1 Flow chart outlining the school recruitment process, group allocation including the number and p within the school and intervention completion rate and matched controls as number and percentage of pupils 


\section{Intervention protocol}

\section{Design}

The exercise programme was designed following formative research which explored adolescents' perceptions of the proposed design, content and delivery of a schoolbased HIIT intervention, the X4A trial: eXercise for Asthma with Commando Joe's (Winn et al., 2017; Appendix P; p. 224-232) in addition to using HIIT guidelines presented within a systematic review (Eddolls et al., 2017). As such, the intervention group followed a 6-month HIIT programme, involving $3 \times 30$-minute sessions per week consisted of multiple bouts of intense exercise lasting between 10-30 seconds, followed by an equal period of rest. In line with Winn et al. (2017) and following the protocol guidelines, with significant input from the research team, Commando Joe's designed the content of the individual sessions and facilitated their delivery within the school. Exercise sessions, the progression of exercise and rest intervals are displayed in Table 5.1.

Table 5.1 Examples of exercise/rest intervals and activities undertaken during those sessions

\begin{tabular}{lc}
\hline & Exercise/Rest Time (s) \\
\hline \multirow{2}{*}{ Week 1-3 } & $10 / 10$ \\
& $10 / 10$ \\
\multirow{2}{*}{ Week 4-6 } & $10 / 10$ \\
\hline \multirow{2}{*}{ Week 7+ } & $15 / 15$ \\
& $15 / 15$ \\
& $15 / 15$ \\
\hline
\end{tabular}

\section{Session structure and content}

To provide as many opportunities for participants to attend as possible, exercise sessions were held both prior to and after school 3 days a week. A Commando Joe's instructor, a fully qualified personal trainer led the sessions. The instructor also had considerable input into the content of the individual sessions, which consisted of sprinting and bodyweight resistance exercises. Detailed examples of exercise sessions completed are displayed in Table 5.2. 
Table 5.2 Detailed examples of exercises

Activity $\quad$ Example exercises

Static exercises Participants stood in front of a designated cone where they conducted exercises such as:

- Jumping jacks

- $\quad$ Squats

- $\quad$ Press-ups

- $\quad$ Burpees

Obstacle course An improvised obstacle course, created using various resources such as camouflage nets, ladders, tyres and hurdles. The courses incorporated sections that included:

- $\quad$ Crawling

- $\quad$ Rolling

- $\quad$ Agility

- Jumping

\begin{tabular}{lcl}
\hline Speed, agility & Activities included fast paced agility exercises such as: \\
and quickness & - & Slalom sprints \\
& • & Shuttles \\
& • & Fast feet ladder drills \\
& Zig-zag sprints \\
& - & Hurdles
\end{tabular}

Linear sprints Participants moved between cones. Movement between cones was varied i.e. forward, backwards and sideways. Variations to the sprints included:

- $\quad$ Bear crawls

- Gorilla walks

- Crab walks

Throughout each exercise bout, participants were asked to exercise maximally with a view to their HR exceeding $90 \%$ age-predicted maximum HR during each exercise bout, according to the formula developed in Tanaka, Monahan and Seals (2001). Although incremental ramps tests are perhaps the leading and most common method to establish a person's maximum HR (Lanzi et al., 2015; Machado \& Denadai, 2011), 
given the number of consenting participants this method of calculating participants' maximum HR was deemed unfeasible. Consequently, although the formula by Tanaka et al. (2001) can often over- or under-estimate maximum HR, possibly jeopardising the benefits of HIIT given that an individual may not be exercising at $90 \%$ of their true maximum HR, it has been previously validated for use in adolescents (Machado \& Denadai, 2011; Mahon, Marjerrison, Lee, Woodruff, \& Hanna, 2010) and was considered the most pragmatic method available. If it became apparent that an activity did not induce the required HR across a number of participants, that activity was not repeated.

To gauge the intensity of specific activities within the exercise session, prior to the start of the session, participants were fitted with Activio Sport (Activio AB, Stockholm, SWE) HR monitors. Participants' HRs were continuously monitored throughout the session with participants encouraged to increase their exercise intensity if they did not achieve the $90 \%$ maximum HR threshold during each exercise bout. Heart rate data for the participants included within this study was analysed using Activio Sport System (Activio AB, Stockholm, SWE). Following the exclusion of the warm-up and cool-down, average HR was calculated as the HR across the whole session. The mean maximum HR was determined using the averaged maximum HRs achieved from each participant from each session. Participants HR's were expressed and recorded as a percentage of the participant's predicted maximal HR.

\section{Measures}

As part of a wider randomised control trial, all participants conducted a battery of assessments with a smaller sub-sample completing additional measures such as blood lipid analysis, blood pressure, pulse wave velocity and incremental ramps tests measuring peak oxygen uptake. However, since these measures were collected as part of a coinciding but separate $\mathrm{PhD}$ project, these measures were not included within this study or within a separate sub-study. Measurements were taken at four-time points (baseline, mid-intervention, post-intervention and follow-up), approximately 3 months apart, across one academic year (September-July). An outline of when testing at the respective schools took place and how the intervention coincided with testing is provided in Table 5.3. 
Table 5.3 Overview of trial timeline

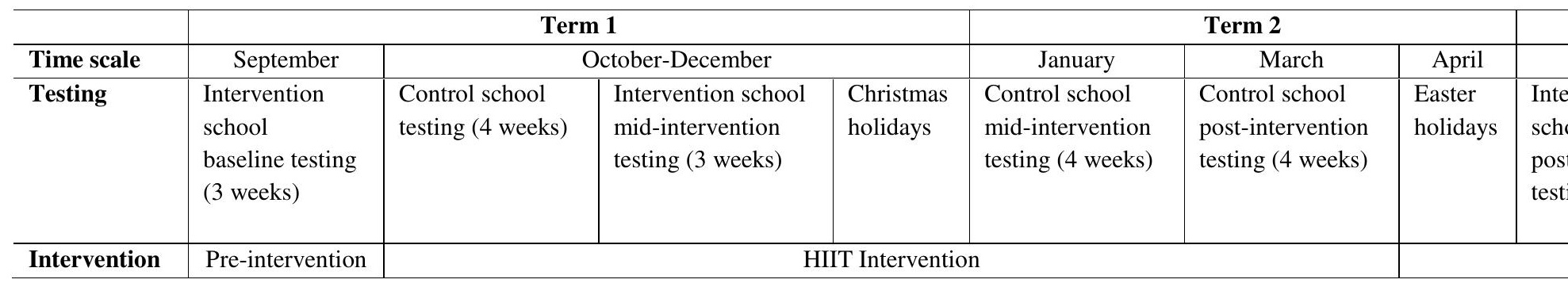




\section{Anthropometrics and maturation}

Participant stature was measured using a potable stadiometer (Seca 213, Seca Ltd, Birmingham, UK), with body mass measured using calibrated body mass scales (Seca 899, Seca Ltd, Birmingham, UK) to the nearest $1.0 \mathrm{~cm}$ and $0.1 \mathrm{~kg}$, respectively. In compliance with the International Standards for Anthropometric Assessment (ISAK) guidelines (Stewart, Marfell-Jones, Olds, \& de Ridder, 2011), during these assessments participants were barefoot and wore light clothing. From these measures, BMI was calculated. Maturational status was assessed using self-reporting indices of pubic hair, as described by Tanner (1962; Table 5.4).

Table 5.4 Anthropometric and maturational data at baseline

\begin{tabular}{lcc}
\hline & $\begin{array}{c}\text { Intervention } \\
(\mathbf{n}=\mathbf{2 7})\end{array}$ & $\begin{array}{c}\text { Control } \\
(\mathbf{n = 5 4})\end{array}$ \\
\hline Height $(\mathbf{m})$ & $1.56 \pm 0.10$ & $1.58 \pm 0.10$ \\
Body mass $(\mathbf{k g})$ & $50.4 \pm 13.3$ & $52.4 \pm 14.1$ \\
Tanner stage & $3.3 \pm 1.1$ & $3.2 \pm 1.1$ \\
\hline
\end{tabular}

\section{Cardiorespiratory fitness}

Cardiorespiratory fitness was estimated using the multi-stage fitness test (MSFT), a previously validated field measure in youth (Boreham et al., 1990; Mayorga-Vega et al., 2015). The MSFT was conducted over a 20-metre space, in a hard-floor indoor sports hall, with the total number of shuttles completed used as a score marker.

\section{Physical activity levels}

The GT3X+ (ActiGraph, Pensacola, FL, USA), a valid and reliable objective measure of physical activity (Trost, 2007), was used to measure and record the quantity, intensity and frequency of body movement. Participants were asked to wear the GT3X+ accelerometer, set at $100 \mathrm{~Hz}$, on their right mid-axilla line at the level of the iliac crest for seven full days, only removing it if they undertook contact or waterbased activities. Wear-time diaries were used to log the reason why the accelerometers were taken off, when, and for how long. Data was analysed using KineSoft (version 3.3.67; KineSoft, Saskatchewan, Canada) employing 1 second epochs, with sustained periods of at least 20 minutes of zero counts considered non-wear-time (Catellier et al., 2005). In order to capture an accurate representation of daily, weekday and 
weekend day physical activity, a minimum daily wear-time of 10 hours for 3 days, including 1 weekend day, was set (Rich et al., 2013). Of the 81 participants who received an accelerometer over 4 time points, the wear-time criteria was met 106 times (25\%). Evenson, Catellier, Gill, Ondrak and McMurray (2008) cut-points, shown to be valid and reliable determinants of activity intensity in adolescents (Trost et al., 2011), were used to calculate the intensity of physical activity.

\section{Questionnaires}

Questionnaire internal consistency and scale reliability were measured using Cronbach's Alpha (Cronbach, 1951) and assessed for quality using previously specified criteria (Field, 2013).

\section{Anxiety}

The 7-item Generalised Anxiety Disorder 7 (GAD-7) assessment (Spitzer et al., 2006; Appendix Q; p. 233), was utilised to measure symptoms of anxiety. Although other questionnaires are potentially more valid and have been used more commonly these alternative questionnaires, such as the State Trait Anxiety Inventory - Children (STAIC; Spielberger \& Edwards, 1973), the Beck Anxiety Inventory (BAI; Beck et al., 1988), were not considered time or cost efficient for the present study. In contrast, the GAD-7, which has previously been used (Wong et al., 2014) and validated in the general population (Löwe et al., 2008) and in adolescents (Mossman et al., 2017) represented a time-efficient and practical measure of anxiety. Participants responded to questions related to general anxiety (e.g., Over the past 2 weeks have you been... feeling afraid as if something awful might happen?) using a 4-point scale $(0=$ Not at all; 3 = Nearly every day). The overall summed scores of the seven items ranged from 0-21, with higher scores indicating a greater severity of general anxiety. Internal consistency for this measure was deemed to be good $(\alpha=0.88-0.89)$.

\section{Depression}

The Center for Epidemiologic Studies Depression Scale for Children (CES-DC; Weissman et al., 1980) was used to assess levels of depression. Whilst the Hamilton Rating Scale for Depression (HAM-D; Hamilton, 1960) and the Beck Depression Inventory (BDI; Beck et al., 1961), are perhaps more valid measures of depressive symptoms these scales were not practical. Given that HAM-D requires a trained 
clinician to administer the test on an individual basis and the BDI is copyrighted and requires a fee for each copy used these scales were deemed unfeasible. In comparison, the CES-DC is self-reporting and free to use, and has been validated for use within the adolescent population (Binagwaho et al., 2012; Fendrich et al., 1990; Stockings et al., 2015). The CES-DC is a 20 -item scale in which participants respond to statements relating to indicators of depression (e.g., During the past week... I have felt down and unhappy). Overall scores range from $0-60$ based on a 4-point scale $(0=$ Not at all, $3=$ A lot), with higher scores representing a greater level of depression. Cronbach's Alpha coefficient scores for CES-DC $(\alpha=0.88-0.94)$ were good.

\section{Quality of life}

The Pediatric Quality of Life Inventory (PedsQL) Teenager Report (Version 4.0) was used to measure QoL, and is a widely validated measure in adolescents (Varni et al., 2006, 2003, 2001). Although the 57-item KIDSCREEN (Ravens-Sieberer et al., 2005) has been shown to provide similar validity and reliability (Janssens et al., 2008), given time-constraints and the fewer number of items and smaller estimated completion time, the PedsQL was deemed the most pragmatic choice. Indeed, the PedsQL is a 23-item scale in which participants were asked to respond, using a 5-point scale $(0=$ Never, 4 $=$ Almost always), to statements relating to 4 sub-scales: physical $(n=8)$, emotional $(\mathrm{n}=5)$, social $(\mathrm{n}=5)$ and school functioning $(\mathrm{n}=5$; e.g., I have trouble sleeping). Scores can range between 0-100, with higher scores indicating a better QoL. Scale reliability for this measure $(\alpha=0.88-0.92)$ was deemed acceptable.

\section{Statistical analysis}

Using SPSS version 22 (IBM Corp, USA), data were analysed using a mixed measures analysis of variance (ANOVA) with groups as the between- and time as the withinsubject effects. Partial eta-squared $\left(\eta_{\mathrm{p}}^{2}\right)$ effect size was calculated from baseline to follow-up and classified in line with J. Cohen $(1988,1992)$ guidelines for effect sizes ( small $=0.2$, medium $=0.5$, large $=0.8)$. All data is presented as mean \pm standard deviations, with significance accepted at $p<0.05$.

\section{Results}

Presented as percentages, data regarding the number of participants for which data was obtained at each time point are presented in Table 5.5. For all parameters, except 
physical activity, the main reason for missing data was participants being absent from school or unavailable when data collection was taking place. Specifically, for physical activity, there was poor wear-time compliance and therefore the physical activity data of all participants not meeting the wear-time criteria were excluded.

There were no significant differences in sex, maturation, anthropometric measures, cardiorespiratory fitness, physical activity, symptoms of anxiety or depression or QoL between groups at baseline (Table 5.6). For the intervention group, mean scores over time suggest that BMI, cardiorespiratory fitness, anxiety, depression and QoL improved post-intervention, before subsequently demonstrating increased symptoms of depression and anxiety and a decline in QoL at follow-up. Conversely, mean moderate-to-vigorous physical activity levels decreased over time. However, for all parameters, these changes failed to reach statistical significance. There were no significant differences in depression, anxiety or QoL between sexes or according to maturity $(p>0.05)$.

\section{Body mass index}

The classification of body composition based on terminology by Barlow and the Expert Committee (2007) suggested that, within the intervention group, 78\% were normal weight $\left(5-85^{\text {th }}\right.$ percentile $), 15 \%$ overweight $\left(85-95^{\text {th }}\right.$ percentile) and $7 \%$ also classified as obese $\left(>95^{\text {th }}\right.$ percentile). Within the control group, $68 \%$ were classed as normal weight, $23 \%$ as overweight with a further $9 \%$ classed as obese. Although no significant interaction was detected between the intervention and control groups and time for $\operatorname{BMI}\left(F(2.08,162.16)=1.80, p=0.17, \eta_{\mathrm{p}}{ }^{2}=0.02\right)$, a main effect for time $(F$ $\left.(2.08,162.16)=15.17, p=p<0.001, \eta_{\mathrm{p}}^{2}=0.16\right)$ was detected. Specifically, a significant difference was noted between baseline and follow-up (-0.42, 95\% CI, -0.70 to $-0.14, p<0.05)$. However, the main effect for group showed no statistically significant difference $\left.(F(1,78)=0.08), p=0.80, \eta_{\mathrm{p}}{ }^{2}<0.01\right)$. 
Table 5.5 The number of participants for which data was obtained at each time point

\begin{tabular}{|c|c|c|c|c|c|c|}
\hline & & Inter & ention & & & \\
\hline & Baseline & $\begin{array}{c}\text { Mid- } \\
\text { intervention }\end{array}$ & $\begin{array}{c}\text { Post- } \\
\text { intervention }\end{array}$ & Follow-up & Baseline & $\begin{array}{r}\text { Mic } \\
\text { interve }\end{array}$ \\
\hline BMI & $27(100 \%)$ & $27(100 \%)$ & $27(100 \%)$ & $27(100 \%)$ & $54(100 \%)$ & $54(10$ \\
\hline Cardiorespiratory fitness & $27(100 \%)$ & $27(100 \%)$ & $27(100 \%)$ & $26(96.3 \%)$ & $54(100 \%)$ & $46(85$ \\
\hline MVPA & $17(63.0 \%)$ & $15(55.6 \%)$ & $11(40.7 \%)$ & $11(40.7 \%)$ & $25(46.3 \%)$ & $14(25$ \\
\hline GAD-7 & $27(100 \%)$ & $27(100 \%)$ & $27(100 \%)$ & $27(100 \%)$ & $54(100 \%)$ & $54(1$ \\
\hline CES-DC & $27(100 \%)$ & $27(100 \%)$ & $27(100 \%)$ & $27(100 \%)$ & $54(100 \%)$ & $54(1$ \\
\hline PedsQL & $27(100 \%)$ & $27(100 \%)$ & $27(100 \%)$ & $27(100 \%)$ & $54(100 \%)$ & $54(1$ \\
\hline
\end{tabular}




\section{Cardiorespiratory fitness}

According to the thresholds proposed by Boddy, Thomas, et al. (2012), 8 participants from the intervention group and 9 participants from the control were potentially at risk of cardiometabolic disease at baseline. There was no significant interaction between group and time for cardiorespiratory fitness $\left(F(3,147)=2.60, p>0.05, \eta_{\mathrm{p}}{ }^{2}=0.05\right)$, with no main effect for time $\left(F(3,147)=1.61, p=0.19, \eta_{\mathrm{p}}{ }^{2}=0.03\right)$. Additionally, there was no significant difference between groups at baseline $(F(1,49)=0.02), p=$ $\left.0.89, \eta_{\mathrm{p}}^{2}<0.01\right)$.

\section{Moderate-to-vigorous physical activity}

At baseline, within the intervention group only 6 of the $17(35.3 \%)$ that met the weartime criteria also achieved the government guidelines of 60 minutes of moderate-tovigorous physical activity (Department of Health, 2011). In the control group, 8 of the $25(32 \%)$ that met the wear-time criteria accumulated greater than 60 minutes moderate-to-vigorous physical activity. No significant interaction between group and time for moderate-to-vigorous physical activity was detected $(F(3,12)=1.34, p>$ $\left.0.05, \eta_{\mathrm{p}}{ }^{2}=0.25\right)$. Additionally, there was no main effect of time $(F(3,12)=2.50, p=$ $\left.0.11, \eta_{\mathrm{p}}^{2}=0.38\right)$ or group $\left(F(1,4)=0.17, p=0.71, \eta_{\mathrm{p}}^{2}=0.04\right)$.

\section{Anxiety}

There was no significant interaction between group and time for anxiety ( $F$ (2.43, $\left.189.78)=1.12, p>0.05, \eta_{\mathrm{p}}{ }^{2}<0.01\right)$. Additionally, there was no main effect of time $\left(F(2.43,189.78)=0.43, p=0.73, \eta_{\mathrm{p}}{ }^{2}<0.01\right)$ or $\operatorname{group}\left(F(1,78)=1.79, p=0.17, \eta_{\mathrm{p}}{ }^{2}\right.$ $=0.02$ ).

\section{Depression}

For depression, there was no significant interaction between group and time $(F(3,234)$ $\left.=0.29, p=0.83, \eta_{\mathrm{p}}^{2}<0.01\right)$, also there was no main effects for time $(F(3,234)=0.36$, $\left.p=0.78, \eta_{\mathrm{p}}^{2}<0.01\right)$ or group $\left(F(1,78)=3.20, p=0.08, \eta_{\mathrm{p}}{ }^{2}=0.04\right)$.

\section{Quality of life}

There was no significant interaction between group and time for $\operatorname{QoL}(F(3,231)=$ $\left.1.17, p>0.05, \eta_{\mathrm{p}}^{2}=0.02\right)$. However, there was a main effect for time $(F(3,231)=$ $\left.2.73, p<0.05, \eta_{\mathrm{p}}{ }^{2}=0.03\right)$ and group $\left.(F(1,77)=4.12), p<0.05, \eta_{\mathrm{p}}{ }^{2}<0.05\right)$. Despite this, for time the reported significant differences were not in relation to group. 
Table 5.6 Intervention and control group BMI, cardiorespiratory fitness, MVPA, anxiety, depression and qua deviation over time

\begin{tabular}{|c|c|c|c|c|c|c|}
\hline \multicolumn{7}{|c|}{ Intervention } \\
\hline & Baseline & $\begin{array}{c}\text { Mid- } \\
\text { intervention }\end{array}$ & $\begin{array}{c}\text { Post- } \\
\text { intervention }\end{array}$ & Follow-up & Baseline & \\
\hline BMI & $20.3 \pm 3.4$ & $20.1 \pm 3.5$ & $20.5 \pm 3.6$ & $20.8 \pm 3.5$ & $20.6 \pm 3.6$ & \\
\hline MSFT shuttles & $48.4 \pm 24.6$ & $58.8 \pm 22.7$ & $56.7 \pm 20.6$ & $53.8 \pm 24.6$ & $52.3 \pm 25.8$ & 51 \\
\hline MVPA (mins) & $56 \pm 14$ & $46 \pm 10$ & $47 \pm 11$ & $46 \pm 21$ & $56 \pm 25$ & \\
\hline GAD-7 & $4.2 \pm 4.9$ & $3.6 \pm 3.4$ & $3.9 \pm 3.6$ & $4.4 \pm 4.3$ & $5.2 \pm 4.9$ & 4 \\
\hline CES-DC & $15.0 \pm 9.6$ & $14.7 \pm 9.2$ & $14.2 \pm 8.7$ & $14.7 \pm 8.3$ & $19.5 \pm 11.1$ & 19.5 \\
\hline PedsQL & $82.7 \pm 12.1$ & $81.6 \pm 11.6$ & $84.7 \pm 10.7$ & $84.4 \pm 9.8$ & $76.8 \pm 14.0$ & 74. \\
\hline
\end{tabular}


Additionally, although significant differences were noted for group, they were between the same intervention protocol.

\section{Heart rate data}

Excluding the warm-up and cool-down periods, participants' mean HR $(155 \pm 18$ beats per minute (bpm), $78 \pm 9 \%$ maximum HR) and mean maximum HR (188 \pm 18 bpm, $95 \pm 6 \%$ maximum HR) were calculated for each session. During the main body of the session, inclusive of both the exercise and rest intervals due to the inability to definitively identify individual exercise bouts, participants' HR exceeded the threshold of $>90 \%$ maximum HR $24 \pm 24 \%$ of the time.

\section{Discussion}

The present study investigated the effect of a 6-month school-based HIIT intervention on BMI, cardiorespiratory fitness, physical activity levels, depression, anxiety and QoL in adolescents. The main findings were that HIIT did not significantly improve BMI, cardiorespiratory fitness, physical activity levels, depression, anxiety or QoL. The lack of adaptation suggests that HIIT exercise was not associated with a positive effect on physical or mental health in adolescents.

Associated to HIIT (Costigan et al., 2017), VPA has previously been shown to improve mental health and QoL (Eddolls et al., 2018), however, contrary to this, the present study indicated that HIIT did not improve anxiety, depression or QoL in adolescents. Whilst the lack of significant findings are likely a result of the small sample size and the subsequent implications on statistical power, additional factors may also have attributed to such findings. Specifically, it could be postulated that such results are attributable to the homogeneity of the population within this study. At baseline, the intervention group's mean scores for the depression and anxiety measurements were relatively low in relation to their respective scales. Equally, for the QoL measurement, the cohort's mean baseline score was also relatively high. Consequently, there may have been a ceiling effect, whereby significant results may not have been attainable due to the already healthy state of mental health and QoL. Indeed, recent research that explored the effects of $\mathrm{CrossFit}^{\mathrm{TM}}$ on mental health in adolescents found that, although no significant improvements were manifest as a result of the high-intensity exercise in the full study sample, participants categorised as 'at risk' of psychological distress demonstrated significant improvements (Eather, Morgan, \& Lubans, 2016). 
Specifically, despite not directly aligning with the measures within the present study, those 'at risk' reported improvements in self-esteem, perceived body fat, perceived appearance and physical self-concept. It is therefore suggested that future research explore the effects of HIIT in those considered to be at risk of developing mental health issues.

Similar to the recent findings of Costigan et al. (2016), who also found that HIIT does not improve mental health, these findings may also be attributable to factors relating to the exercise protocol. Specifically, whilst this study followed the protocol guidelines developed in a recent systematic review (Eddolls et al., 2017), these guidelines were based on the intervention design associated with the greatest physiological benefit; exercise protocols for psychosocial variables may therefore differ. Given that previous research has demonstrated vigorous physical activity (VPA) and HIIT can significantly improve mental health (Eddolls et al., 2018; Gerber et al., 2014; Parfitt et al., 2009; Shepherd et al., 2015), in line with past recommendations (Costigan et al., 2016), future research should establish a HIIT intervention protocol based on psychological outcomes. Indeed, it has been suggested that social interactions inherent to exercise may induce psychological improvements (Biddle \& Asare, 2011; Helseth \& Misvær, 2010). Therefore, although running-based exercises may benefit physiological measures (Eddolls et al., 2017), the individual nature of this type of exercise may not be conducive to peer interaction. Conversely, team-based games, such as those in K. L. Weston et al. (2016), may be more appropriate for improvements to mental health and QoL.

In conjunction with factors relating to the exercise protocol, the lack of significant improvements in anxiety, depression and QoL across all time-points, may be related to the intervention components. Whilst previous research has demonstrated that HIIT, as a singular intervention component, elicits improvements in the mental health of adults (Shepherd et al., 2015), such an approach may not be applicable for adolescents. Indeed, the apparent age-related discrepancies between the findings may, in part, be due to differing levels of experience in coping with symptoms of poor mental health and QoL, with adults better prepared to manage such feelings (Aldwin, 1991). As such, developing an intervention in which HIIT is supplemented by multiple components, such as psychological or educational elements (S. Reynolds, Wilson, Austin, \& Hooper, 2012; Weisz, McCarty, \& Valeri, 2006), that can also engender improvements 
in the mental well-being and QoL of adolescents should be considered. Indeed, psychological or educational interventions combined with exercise have been shown to significantly improve both depression and anxiety (Coventry et al., 2013; Parker et al., 2016). It has been proposed that exercise may afford a person meaningful mastery experience, allowing the opportunity to practice techniques learned via psychological therapy to boost their perceived ability to cope with depression (Craft, 2005). Consequently, HIIT may provide a time-effective platform, when compared to highvolume moderate-intensity exercise, from which to develop coping abilities.

Whilst several methodological and statistical explanations for the lack of significant findings have been hypothesised, such findings may simply be attributable to the lack of increase or maintenance in total physical activity. Although speculative, given that the poor wear-time compliance of the accelerometers and subsequent questionable reliability, it could be postulated that whilst the present study sought to enhance VPA, the HIIT intervention did not increase adolescents' total physical activity. Indeed, in accord with previous literature, total physical activity has been associated with a greater QoL (Wafa et al., 2016) and fewer symptoms of depression and anxiety (J. R. Andersen et al., 2017; Kremer et al., 2014; McMahon et al., 2017). It is therefore possible that the current findings may reflect a "compensation effect" (Ridgers et al., 2014), whereby intervention participants compensate for an elevated level of physical activity on one day with a reduction in physical activity levels the following day. Consequently, increases accrued during the HIIT session were potentially negated by reduced physical activity outside of the exercise sessions. Accordingly, to detect and account for possible alterations in habitual physical activity that may confound any beneficial effects to mental well-being or QoL, future research should ensure that physical activity is tracked throughout any prospective interventions.

It could be speculated that the lack of intervention effect was attributable to the HIIT intervention, which did not elicit improvements to BMI or cardiorespiratory fitness, both of which have been shown to significantly impact depression, anxiety and QoL. Indeed, increased cardiorespiratory fitness has been associated with higher levels of QoL (J. R. Andersen et al., 2017) and has also been shown to protect against the development of mental health issues (Ruggero et al., 2015). Moreover, improved body composition can elicit improvements to self-image and self-esteem (C. E. Ross, 1994), which have both been associated with mental health (Lubans et al., 2016; Markowitz 
et al., 2008). Potential explanations for the lack of significant improvements may, however, be a result of the measures used. For example, given the inherent problems associated with the MSFT, such as motivating adolescents to perform at maximally (Hamlin et al., 2014), it is unclear if the results collected were a true reflection of their cardiorespiratory fitness. Regarding the lack of improvements to BMI, it should be noted that, at baseline, the majority of intervention participants were already considered to be of normal weight. Consequently, reducing BMI may not have been possible given the possibility of a ceiling effect. Furthermore, given that many of the sessions were based around body weight resistance exercises, an increase in muscle mass and subsequent healthy increase in body mass and BMI cannot be dismissed. Regardless, without significantly improving such physiological outcomes, it is unlikely that an exercise intervention will improve mental health or QoL.

Further to the psychological and physical outcomes of the study, considering that the HIIT intervention was largely implemented as planned, it is somewhat surprising that there were no significant intervention effects for anxiety, depression or QoL. Subsequently, it is possible that the lack of significance, at least in part, may be due to the relatively small sample size and thus decreased statistical power. Moreover, it is important to acknowledge that 223 participants initially agreed to take part. Therefore, despite formative research (Winn et al., 2017), this suggests that HIIT or indeed the way this specific HIIT intervention was delivered was not appealing to the target population. It is therefore important to consider capturing the views of participants on the barriers and facilitators to initial and continued participation, consequently providing a useful insight into the efficacy of HIIT.

Although this study has various strengths, there are several limitations. Despite the HR results being comparable to those recently reported (Costigan et al., 2016), it is important to acknowledge that, exclusive of the warm-up and cool-down, this was measured as an average across the whole session. Consequently, the ability to identify the maximum HR achieved during each exercise bout was impeded, though, due to synchronisation issues during the intervention phase, distinguishing between the exercise and rest bouts was not possible. As such, in conjunction with the large standard deviation, it is possible that the participants within this study may not have adhered to the required intensity, which may, at least in part, explain the lack of intervention effect. Beyond the HR results, other measurement tools utilised within 
this thesis are also possibly limited. Specifically, gold standard rating scales (Cusin et al., 2010), such as the HAM-D (Hamilton, 1960) and the BDI (Beck et al., 1961), were not used due to licensing costs and the requirement for a qualified clinician to administer them. However, it should be acknowledged that all mental health measurement tools were validated in the target population (Binagwaho et al., 2012; Fendrich et al., 1990; Löwe et al., 2008; Stockings et al., 2015; Varni et al., 2006, 2003, 2001; Wong et al., 2014). Nonetheless, the self-reported nature of the questionnaires have inherent disadvantages, such as social desirability, which can potentially lead to a participants undervaluing measurement variables (Paulhus \& Vazire, 2005). Despite this, all questionnaires provided acceptable internal consistency, and were therefore deemed reliable. Additional methodological issues, such as of the heterogeneity of the study population may have also compounded the results. Given that the intervention group demonstrated low symptoms of depression and high QoL in comparison to the control group, it could be postulated that intervention effects may have been masked. Consequently, controlling for the baseline values may have ameliorated such issues. However, this type of analysis was deemed unsuitable given that this study utilised repeated measures and would assume that all error terms were independent, which was not the case.

\section{Conclusion}

In conclusion, despite the well-documented physiological and psychological benefits elicited by HIIT in previous studies, the present findings do not support HIIT as a tool to enhance adolescent BMI, cardiorespiratory fitness, physical activity, depression, anxiety or QoL. Whilst there are several limitations that may have confounded these results, poor intervention adherence and subsequent small sample size may have limited the statistical power. As such, to gain an appreciation of participants views, Chapter 6 will explore adolescents' perceptions of the X4ACJ HIIT intervention and elicit their understanding of the barriers and facilitators to initial and continued participation. 


\section{Thesis study map}

\begin{tabular}{|c|c|c|}
\hline Study & & Outcomes \\
\hline $\begin{array}{l}\text { The association } \\
\text { between physical } \\
\text { activity, fitness and } \\
\text { body size and their } \\
\text { effect on mental } \\
\text { well-being and } \\
\text { quality of life in } \\
\text { children and } \\
\text { adolescents }\end{array}$ & Key Findings & $\begin{array}{l}\text { To investigate the relationships between } \\
\text { VPA, BMI and cardiorespiratory fitness on } \\
\text { symptoms of depression, and their } \\
\text { subsequent direct and indirect effects on QoL } \\
\text { in children and adolescents } \\
\text { Vigorous physical activity, cardiorespiratory } \\
\text { fitness and BMI are associated, both directly } \\
\text { and indirectly, with mental well-being and } \\
\text { QoL in children and adolescents. }\end{array}$ \\
\hline $\begin{array}{l}\text { The effect of high- } \\
\text { intensity interval } \\
\text { training on mental } \\
\text { well-being and } \\
\text { quality of life in } \\
\text { adolescents: the } \\
\text { eXercise for Asthma } \\
\text { with Commando } \\
\text { Joe's }{ }^{\circledR} \text { (X4ACJ) trial }\end{array}$ & Key Findings & $\begin{array}{l}\text { To assess the short-term and longer-term } \\
\text { effects of a school-based HIIT intervention } \\
\text { on body mass index, cardiorespiratory } \\
\text { fitness, physical activity levels, depression, } \\
\text { anxiety and QoL in adolescents. } \\
\text { HIIT does not significantly improve BMI, } \\
\text { cardiorespiratory fitness, physical activity, } \\
\text { depression, anxiety or QoL in adolescents. }\end{array}$ \\
\hline $\begin{array}{l}\text { Exploring adolescent } \\
\text { perceptions of a } \\
\text { high-intensity } \\
\text { interval training } \\
\text { intervention: } \\
\text { evaluating the } \\
\text { eXercise for Asthma } \\
\text { with Commando } \\
\text { Joe's }{ }^{\circledR}(\mathrm{X} 4 \mathrm{ACJ}) \text { trial }\end{array}$ & Key Findings & $\begin{array}{l}\text { To explore adolescents' perceptions of the } \\
\text { eXercise for Asthma with Commando Joe's }{ }^{\circledR} \\
\text { (X4ACJ) HIIT intervention and to elicit their } \\
\text { views on the barriers and facilitators to initial } \\
\text { and continued participation. }\end{array}$ \\
\hline
\end{tabular}


Chapter 6 - Exploring adolescent perceptions of a high-intensity interval training intervention: evaluating the eXercise for Asthma with Commando Joe's ${ }^{\circledR}$ (X4ACJ) trial (Study 3)

\section{Introduction}

The prevalence of poor health in adolescents has reached unprecedented levels (Collishaw et al., 2010; Lobstein et al., 2015; Ng et al., 2014). Common limitations to conventional methods for treating such low health are that they are often expensive (McCrone et al., 2008) and time-consuming (Gladstone et al., 2011). Whilst exercise has been identified as a cost-effective tool for health promotion, traditional strategies have principally utilised moderate-intensity continuous exercise (Hansen et al., 1991; McMurray et al., 2002; Meyer et al., 2006). However, questions have been raised regarding the relevance to the sporadic, high-intensity nature of adolescent's habitual play patterns (Brodersen et al., 2007).

High-intensity interval training (HIIT) has been identified as a time-efficient form of exercise that could improve physical (Costigan, Eather, Plotnikoff, Taaffe, \& Lubans, 2015; Eddolls et al., 2017; Logan et al., 2014) and mental health (Shepherd et al., 2015). Despite numerous studies conducting HIIT interventions in adolescents, they have been conducted with varied success (Costigan et al., 2016; Lambrick et al., 2016; Lau et al., 2014; Racil et al., 2016; Tjønna et al., 2009; K. L. Weston et al., 2016). Research to date has attributed this to a low final sample size and its associated implications on statistical power (Moher, Dulberg, \& Wells, 1994), yet few studies have sought to ascertain why there was such poor adherence with regard to attending the sessions, despite being a fundamental aspect of all interventions (Sluijs, Kok, \& van der Zee, 1993).

Given that enjoyment during exercise exhibits a greater affective response (Raedeke, 2007), it could be postulated that such variance was manifest due to differing perceptions of HIIT between studies. Indeed, although there is some evidence that is supportive of the health benefits of HIIT, if participants are unlikely to adhere to interventions in terms of participation, the efficacy of such exercise should be questioned. For example, within Study 2 (p. 86-106) despite 223 participants initially agreeing to take part, only 27 participants attended a sufficient number of sessions $(>70 \%)$ to warrant inclusion in this study. This suggests that HIIT, or the way the eXercise for Asthma with Commando Joe's ${ }^{\circledR}$ (X4ACJ) HIIT intervention was 
delivered, was not appealing. Therefore, the importance of addressing such adherence issues to inform future interventions and identify whether HIIT is an efficient form of health-promotion in adolescents is clear.

Qualitative process evaluation has previously been suggested as a method to explain unexpected quantitative results, such as those in Study 2 (Abildgaard, Saksvik, \& Nielsen, 2016). However, whilst the importance of capturing participants' postintervention attitudes is evident (Hoffmann et al., 2014), to date, there remains a dearth of evaluative research, possibly due to the time-consuming nature of qualitative data collection and analysis. Indeed, whilst two studies have previously explored the feasibility of HIIT through post-intervention focus-groups (Buchan et al., 2013) and questionnaires (Costigan, Eather, Plotnikoff, Taaffe, Pollock, et al., 2015), these process evaluations were limited. Specifically, the questionnaire and focus-group were only included as small secondary measures embedded within wider papers primarily aiming to access the physiological effectiveness of HIIT. Consequently, to maximise the health benefits and participation to exercise, further investigation into participants' perceptions with regard to reasons they enjoy or dislike HIIT is warranted.

Therefore, the aim of this study was to explore adolescents' perceptions of the eXercise for Asthma with Commando Joe's ${ }^{\circledR}$ (X4ACJ) HIIT intervention and to elicit their views on the barriers and facilitators to initial and continued participation.

\section{Methods}

This research was underpinned by ontological relativism and epistemological constructionism. Accordingly, it is assumed that reality is multiple, created and mind dependent, with knowledge being constructed and subjective. A qualitative approach was adopted so that participants were not limited in what they could express, enabling them, in their own words, to communicate their thoughts and perceptions in a more indepth and complex manner.

\section{Participants}

Eighty adolescents $(13.3 \pm 1.0$ years; 45 boys $)$, from the X4ACJ trial, participated in this study. The X4ACJ trial involved a HIIT intervention delivered by a qualified personal trainer from Commando Joe's with previous experience leading group exercise sessions. Participants were asked to attend 3 x 30-minute sessions per week 
prior to or after school for 6 months, with the aim of promoting physiological health and mental well-being. The exercise sessions consisted of multiple bouts of intense exercise lasting between 10-30 seconds, followed by an equal period of rest. During the exercise phase, the exercises were designed to induce a heart rate (HR) exceeding 90\% participants' maximum HR. Participants were either classified as an attendee (n $=36 ; 13.2 \pm 1.0$ years; 23 boys $)$ or a non-attendee $(n=44 ; 13.5 \pm 1.1$ years; 22 boys $)$ if they were actively engaged with the intervention for a sustained period, or attended less than 5\%, of the intervention sessions, respectively. Ethical approval was granted by the institutional research ethics committee (PG/2014/29).

\section{Procedure}

Eighteen semi-structured focus-groups were conducted in groups sizes of 3-6 (Gibson, 2007; Mackintosh, Knowles, Ridgers, \& Fairclough, 2011; Morgan, Gibbs, Maxwell, $\&$ Britten, 2002), except for one interview consisting of only two participants due to pragmatic reasons; no difference between emergent themes were detected and therefore it was deemed appropriate for inclusion. Although there are some disadvantages to using focus-groups with adolescents, such as difficulty in controlling and managing the groups, irrelevant discussions distracting from the main focus and dominant voices (Leung \& Savithiri, 2009; Smithson, 2000), ensuring that the group sizes were small, and subsequently easily controllable, obviated such issues. Indeed, if small with regard to participants numbers, focus-groups with adolescents have been shown to be viable methods by which to explore perspectives (Porcellato, Dughill, \& Springett, 2002). Furthermore, small focus-groups have been recommended for research with young people in order to generate good-quality data (Morgan et al., 2002). Nonetheless, it is important to note that certain participants in focus-groups may be hesitant to express their thoughts, especially when those thoughts oppose the views of others. To circumvent this concern, in addition to being grouped according to attendance, groups were also stratified by academic year group (years 7-10), so that younger participants did not feel intimidated by those in older year groups.

The interviews, facilitated by the lead researcher who had previous experience in conducting focus-groups, were conducted in a quiet space within the school environment (Kennedy, Kools, \& Krueger, 2001) and lasted $45 \pm 7$ minutes. To enable social interaction and observer involvement, participants were positioned around a circular table with WTBE seated amongst the participants (Dilorio, Hockenberry- 
Eaton, Maibach, \& Rivero, 1994; Gibson, 2007). The focus-group questions were structured around eliciting an understanding of the participants' impression of the X4ACJ HIIT intervention, with questions appropriately aligned in accordance with the participants age (Table 6.1). Full interview question scripts for both the attendee and non-attendee groups can be found in Appendices R and S (p. 233-238). All focusgroups were digitally recorded (Samsung Galaxy S7 Edge, Suwon, South Korea).

Table 6.1 Example interview questions

\begin{tabular}{ll}
\hline Section & Examples \\
\hline Session Content & What was your least favourite session and why? \\
& What could we have changed about the sessions to make them \\
more enjoyable? & What made you want to keep coming to sessions? \\
Attendance & How did your impression of the sessions change over time? \\
HIIT & What did you most enjoy about HIIT? \\
Benefits & What did you least enjoy about HIIT? \\
& $\begin{array}{l}\text { Do you think you have had any personal benefits from the } \\
\text { sessions? }\end{array}$ \\
Instructor & What did you think of the Commando Joe's instructor? \\
& How do you think he delivered the sessions?
\end{tabular}

\section{Data analysis}

Data was transcribed verbatim and thematically analysed following V. Clarke and Braun's (2015) process. Analysis began with the lead researcher immersing themself within and familiarising themself with the transcribed data. Following this, using two of the primary methods to identify patterns in thematic analysis (Braun \& Clarke, 2006), the data was both inductively and deductively analysed. Driven by the lead researcher's theoretical interests and epistemological standpoint, data was initially coded deductively or from 'the top down', categorising the data based on broader prehypothesised concepts (instructor, sessions and benefits). Thereafter, however, the broader categorised data was coded inductively from 'the bottom up', based on smaller, but more specific observations that emerged from the data (motivation, 
competition, group size and competing interests etc.). Once all the relevant data had been coded, similar significant broader patterns were clustered into overarching themes. All themes were then reviewed to ensure they were consistent with the narrative of the transcribed data, but also aligned with the aims of the study. Following further refinement of the themes, succinct but informative theme names were defined and then presented using pen profiling.

\section{Data presentation}

Given the need for alternative methodologies within qualitative research to further advance this method within the field of sport and exercise psychology (Biddle, Markland, Gilbourne, Chatzisarantis, \& Sparkes, 2001), key emergent themes were presented as pen profiles. Whilst pen profiling is possibly a less detailed method than standard qualitative research techniques (Ridgers, Knowles, \& Sayers, 2012), it also has its advantages. Indeed, pen profiling is a relatively new method of presenting data that has been shown to be appropriate for representing findings in an intelligible manner for both quantitative and qualitative researchers (Mackintosh et al., 2011; Ridgers et al., 2012).

\section{Trustworthiness of data}

Whilst there is some evidence to support member checking (Lincoln \& Guba, 1985) and the universal criteria, such as those set out by Tracy (2010), given that this research is underpinned by ontological relativism and epistemological constructionism, such measures of trustworthiness were rejected. Indeed, in line with the relativist approach of this study and contradiction to the notion of universal criteria, researchers are unable to make non-socially constructed judgements (J. K. Smith \& Hodkinson, 2009). Furthermore, there is no evidence that member checking can enhance the credibility or trustworthiness of qualitative research (Thomas, 2017). Therefore, consistent with recent qualitative research (D. M. Hill, Carvell, Matthews, Weston, \& Thelwell, 2017), methodological rigour was ensured via the 'critical friends' approach (B. Smith \& McGannon, 2017), whereby the pen profiles, constructed by the lead researcher, were presented to both supervisors who acted as a theoretical sounding board. During this process both supervisors challenged the lead researcher's analytical decisions, encouraging reflection upon the interpretation of the data. 


\section{Results}

In total, four pen profiles were constructed to represent attendees' (Figure 6.1 \& Figure 6.2) and non-attendees' (Figure 6.3Figure 6.4) facilitators and barriers. For narrative analysis, facilitators and barriers for both attendees and non-attendees were combined.

\section{Attendees}

Key emergent themes were structured around the Commando Joe's instructor, the exercise sessions and the benefits of the intervention. Data revealed that participants understood that increased exercise was associated with several benefits, which subsequently facilitated participation in the intervention. Specifically, the attendees identified physiological $(n=29)$ and social benefits $(n=29)$ of the HIIT intervention as leading reasons to attend the sessions:

"For me it was to get fitter... and just to know loads of my friends were going as well". (B21)

Furthermore, attendees $(n=12)$ also suggested that they experienced psychological benefits whilst participating in the X4ACJ trial, which, perhaps also related to the increased sense of achievement suggested by nearly half $(n=17)$ of the adolescents:

"It made me feel better about myself because it felt like I'd achieved something". (B23)

Adolescents who attended felt that the exercise sessions also benefited them in school, as it helped them "get ready for work" (G7) during lessons. Indeed, participants also believed that the X4ACJ intervention improved their vitality $(\mathrm{n}=19)$ and concentration spans $(n=6)$ :

"I would take part again to keep fit... I just feel much better when I'm more fit and more energetic”. (B14)

Aspects of the HIIT sessions themselves emerged as primary facilitators to attendance. The gamification features of the sessions were a leading facilitating factor, with participants suggesting that games motivated their participation $(n=29)$. Furthermore, the games were often competition-based, which the majority $(n=19)$ also believed encouraged further participation:

"The games mainly, because like they were fun and competitive”. (B12) 


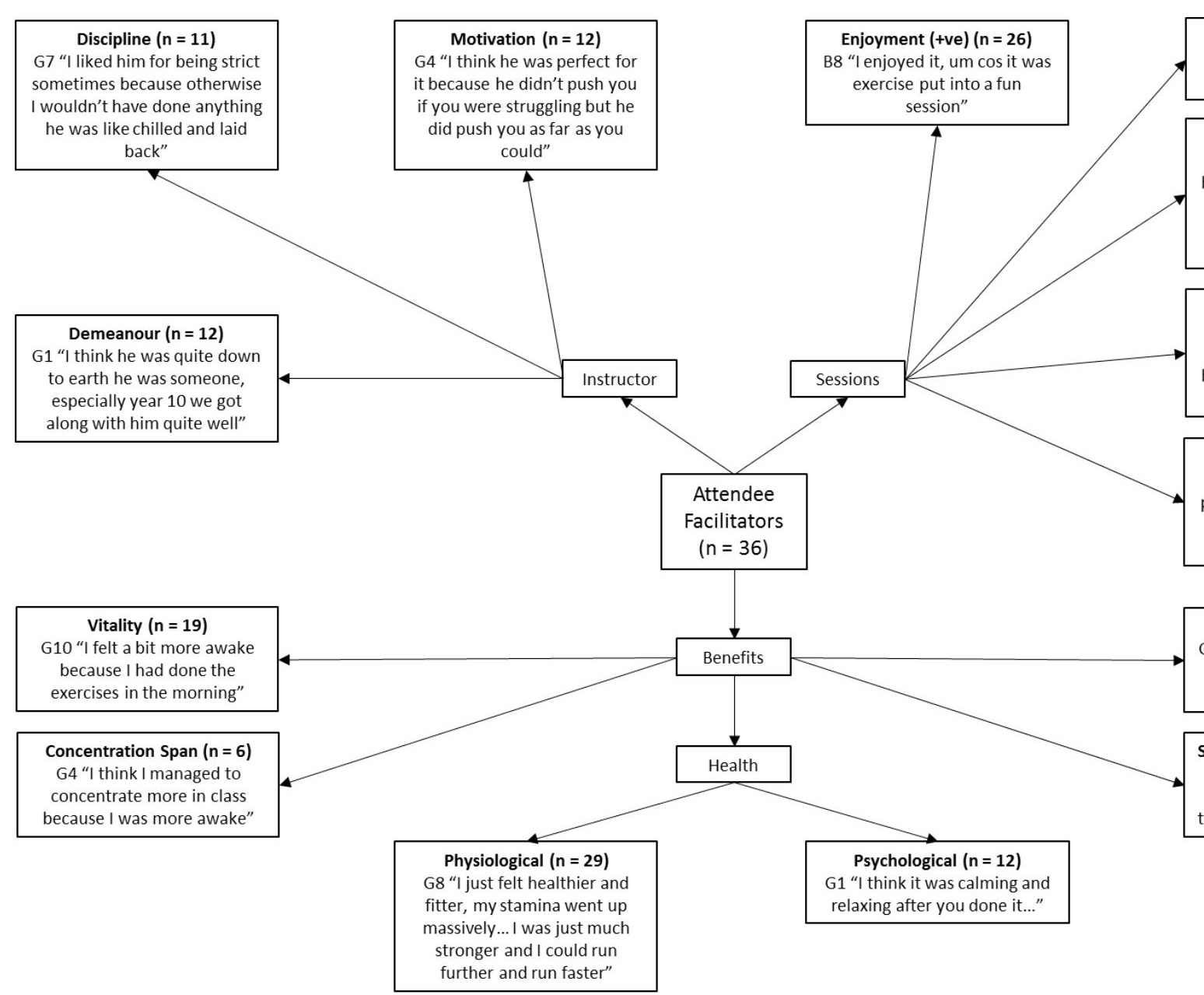

Figure 6.1 Attendee facilitators - pen profile of perceptions of a 6-month high-intensity interval training inter positive 
Additionally, whilst likely linked to games and competition, a large proportion of attendees $(n=26)$ suggested that a reason they attended the exercise sessions was because they enjoyed them:

"I enjoyed it, 'cause it was exercise put into a fun session". (B8)

An additional aspect of the sessions that encouraged adherence in the X4ACJ trial was the challenge of the HIIT exercise. A third of attendees $(n=12)$ expressed that the they enjoyed the challenge of some of the exercises, and that they liked to push themselves:

"It was good to like get pushed, but it was hard as well, so it was good to like reach your limit, so you benefit”. (B22)

Alternatively, however, over half of the attendees $(n=21)$ thought that the sessions were too challenging, indicating that they were being pushed too hard:

“It was pushing us over our limits, so it was too hard”. (B21)

Also, whilst 11 participants suggested that the variation of the session, especially when compared to conventional physical education (PE) lessons, was a facilitating factor, a greater proportion of participants held opposing views, with seventeen participants suggesting that the sessions and exercises were overly repetitive and lacked variation:

"For me it started off good, but then it started going down because it was getting repetitive”. (B14)

Further barriers, such as group sizes were highlighted, with a quarter of participants commenting that overcrowding during the session was an inhibiting factor due to the lack of space:

"I preferred it in the afternoon... the groups were a bit smaller, so you enjoyed it a bit more". (B13)

The type of exercises within the sessions also had a considerable influence on the participants' perceptions of the intervention. Indeed, sprinting- or running-based sessions were described as "boring", with twelve of the attendees perceiving those sessions negatively:

“Constantly running... it was just boring as well, you're not looking forward to just running up and down a sports hall”. (G3) 


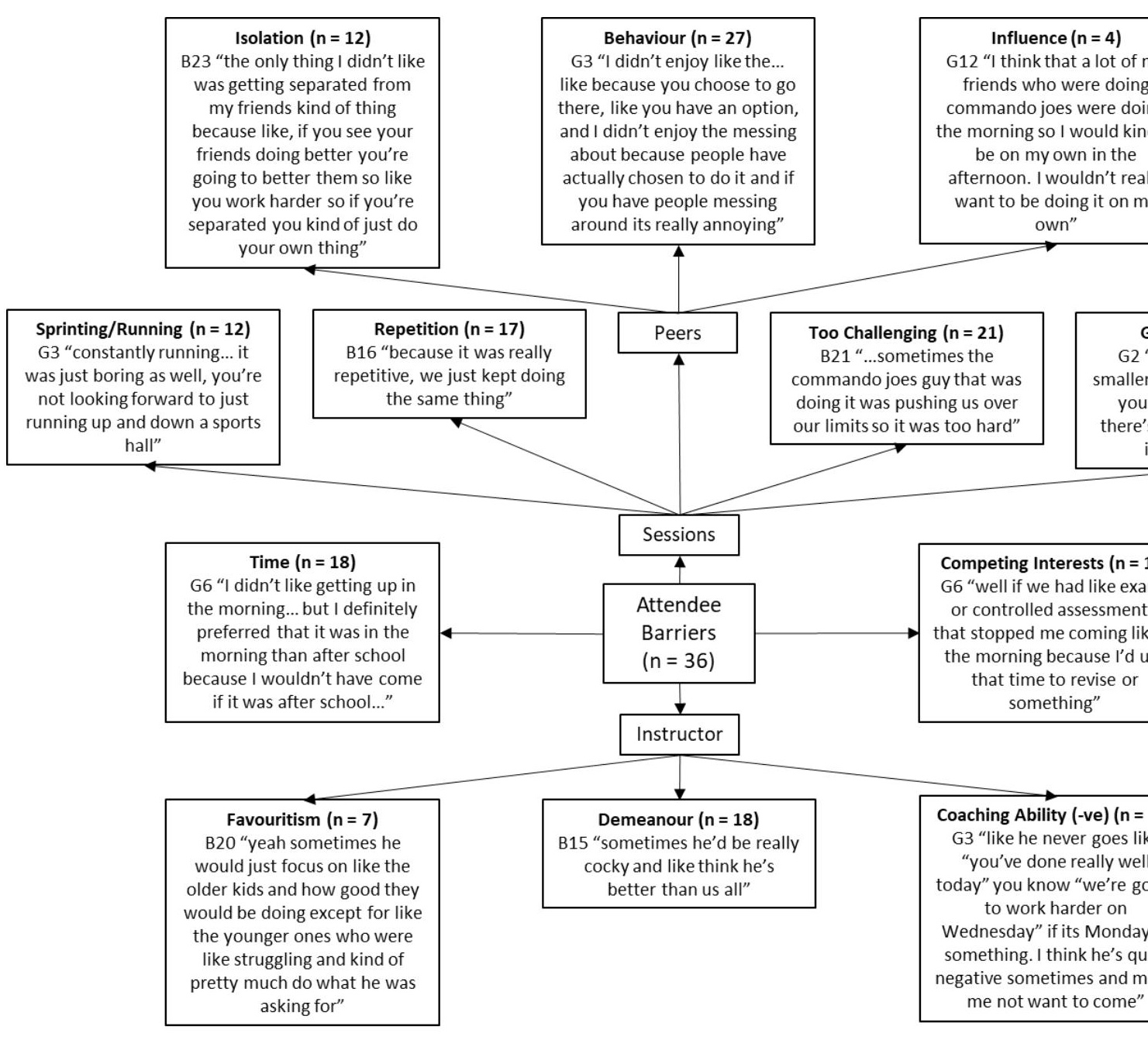

Figure 6.2 Attendee barriers - pen profile of perceptions of a 6-month high-intensity interval training i 
Interestingly, peers emerged as a key influential factor to the X4ACJ exercise sessions. Three-quarters of the attendees commented that disruptive or negative behaviour by other participants within the exercise sessions discouraged further participation:

"I didn't enjoy the messing about because people have actually chosen to do it and if you have people messing around its really annoying”. (G3)

However, peer isolation, the removal from your peer group during the sessions due to disruptive behaviour, was also perceived negatively by 12 attendee participants, despite being used as a strategy to control poor behaviour. Close peers' attendance was also proposed as a potential barrier to participation, with a small proportion of attendees $(n=4)$ stating that they were unlikely to participate if their friends were not attending:

“The only thing I didn't like was getting separated from my friends”. (B23)

The Commando Joe's instructor emerged as both a facilitating and inhibitory factor. Specifically, positive $(n=12)$ and negative $(n=18)$ perceptions of the instructor's demeanour were viewed as a facilitator and barrier, respectively. However, whilst most participants viewed the instructor's demeanour negatively, their motivational abilities were regarded as a dominant facilitator $(\mathrm{n}=12)$, with the discipline kept within the session also acknowledged $(\mathrm{n}=11)$ :

"I liked him for being strict sometimes because otherwise I wouldn't have done anything". (G7)

Conversely, some participants perceived the instructor as a barrier, with a minority of participants $(n=7)$ expressing their belief that the instructor favoured the older year groups, thereby causing resentment. Moreover, although the instructor's motivational abilities were portrayed positively, 13 attendees identified the instructor's coaching ability, or perceived lack of, as a barrier:

"He would just focus on like the older kids and how good they would be doing". (B20)

The timings of the sessions were highlighted as an additional barrier to long-term adherence. Indeed, 18 attendees felt the morning sessions were too early and adolescents were too exhausted to attend after school, with 15 identifying competing interests, such as paper rounds, school work or music lessons, prevented attendance: 


\section{Non-attendees}

For the non-attendees, key emergent themes were also primarily structured around the Commando Joe's instructor, the exercise sessions and the benefits of the intervention. Whilst the attendee group were able to identify several benefits of attending the HIIT sessions, the non-attendee group were only able to recognise the physiological $(n=$ $29)$ and social $(n=16)$ benefits:

"I liked the exercising part to keep fit and it was nice to like do stuff with my friends as well”. (G35)

However, congruent with attendees, over half $(n=23)$ of the non-attendees suggested that games during the sessions facilitated further participation. Whilst distinctly lower than the attendees' group, competitiveness during the sessions was viewed as an encouraging factor by some participants $(\mathrm{n}=8)$. However, despite not attending, a large proportion $(n=21)$ of non-attendees indicated that they enjoyed the sessions:

"I like the competitive side of stuff so any competition you get right into it". (B43)

However, converse to the attendees, fourteen of the non-attendees did not find the sessions enjoyable, and whilst six of the non-attendees enjoyed the variation of the sessions, nine implied that the sessions were repetitive and that these contributed to their non-participation:

"It got boring and because the same stuff was being done all the time". (G20)

Akin to attendees, non-attendees $(\mathrm{n}=6)$ also attributed poor adherence to overcrowding in sessions, suggesting that it prevented them from carrying out the exercises properly, with the variation and the challenge of the sessions also viewed as both a barrier and facilitator. Indeed, although a small proportion $(n=5)$ relished the challenge of the HIIT sessions, a similar amount $(n=7)$ also indicated that the sessions were excessively difficult:

"I found it quite hard because if we pushed like for the full thing and it took a lot of our breath away like if we only had like a 15 second break for example, we're still tired when we go back to doing it". (B34) 

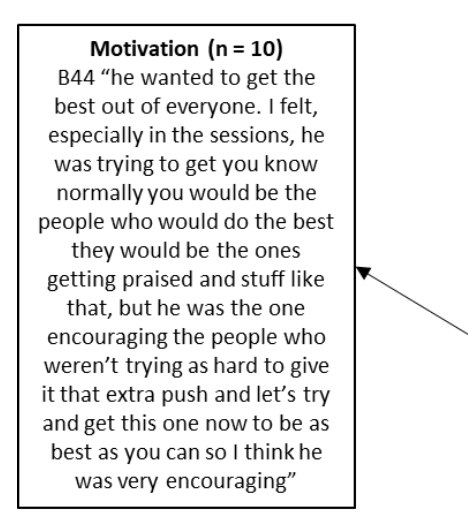

Demeanour ( $n=15)$ B45 "umm, he was sort of umm, we see him as this exarmy sort of hardcore guy but once you get to know him he's quite laid back he... you can talk to him if you have any concerns about certain sports or activities, you can sort of... you feel like you're able to talk to him but also as well as that he's got his sort of... well commandery side so if somethings not been done he will get it done"

Discipline ( $n=5)$ G30 "he kept us on task so there was like no messing about and no one was distracting so we got the work done"

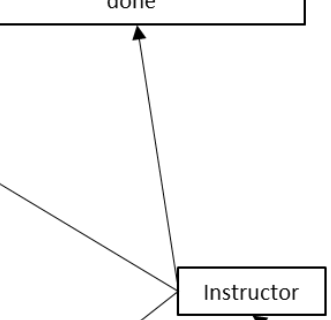

njoyment (+ve) $(n=21)$ B40 "yeah, I enjoyed it I found it interesting"

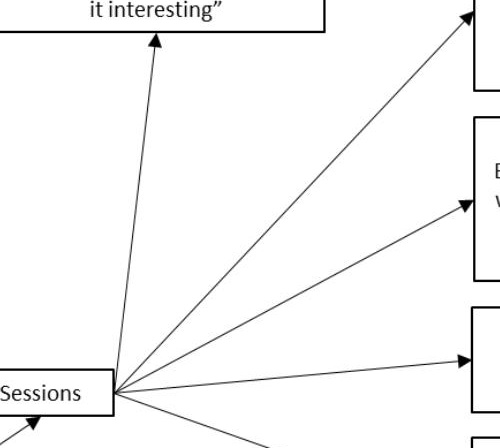

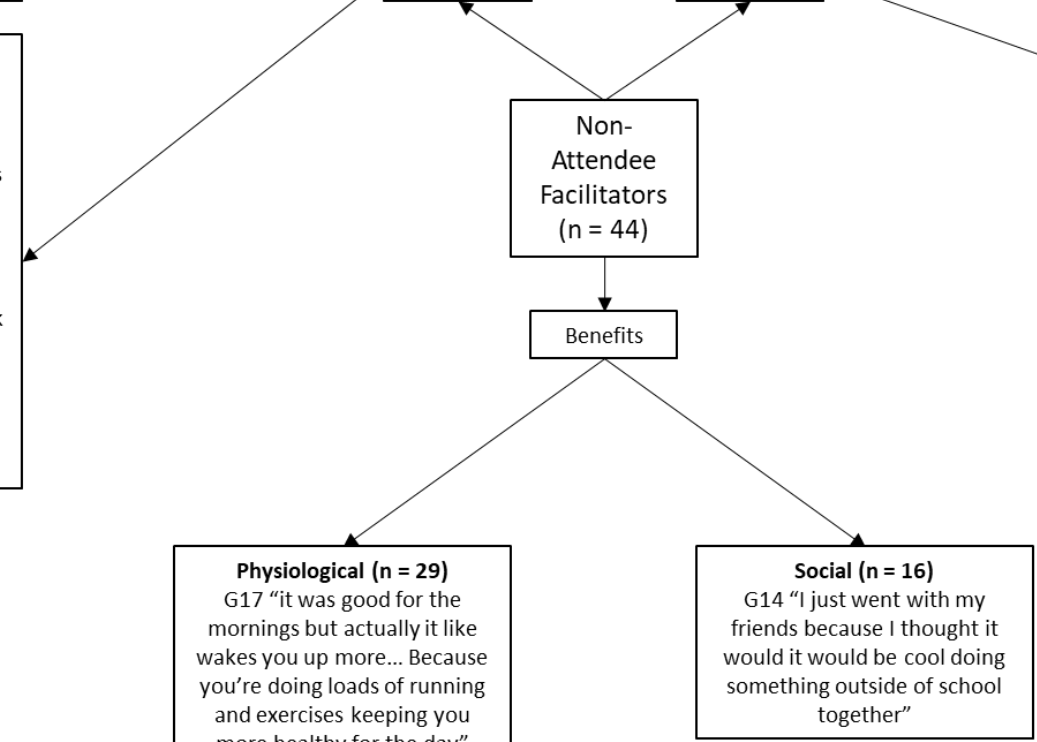

more healthy for the day"

Figure 6.3 Non-attendee facilitators - pen profile of perceptions of a 6-month high-intensity interval training $=$ positive 
Like the attendees, peers were identified as a key influential factor, with a similar proportion of participants $(n=6)$ stating that their close friends' non-attendance was a barrier. However, non-attendees also raised an aversion to exercising with other year groups; over a quarter $(n=12)$ did not want to exercise with either the older or younger year groups:

"It was intimidating [older year groups]”. (G23)

Whilst the attendees expressed mixed perceptions of the instructor, non-attendees were generally more positive. Although seven participants viewed the instructor negatively: "...very intimidating when he's trying to encourage you to get on with it". (B44)

More than double $(n=15)$ suggested that they enjoyed the instructor's positive demeanour. Despite this, although still a facilitating factor, a lower proportion of participants $(\mathrm{n}=5)$ complimented the instructor's disciplinary approach and motivational capabilities $(n=10)$ when compared to the attendee group:

"I like him because when everyone else was in pairs I was on my own, so he got up and went to be my partner... I felt like I worked harder because I had to beat him". (G27)

Although non-attendees expressed numerous facilitators, consistent with attendees, time of the sessions $(n=18)$ and competing interests $(n=21)$ were also raised as key barriers:

"I didn't have time because before school I have to do a paper round... well I have football training and rugby training, so I just can't fit it in really”. (B40)

However, an additional factor that was not suggested within the attendee group was a lack of motivation. Indeed, 12 of non-attendees indicated that they simply could not be bothered:

"I purely couldn't be bothered... it's too early in the morning... and I just couldn't be bothered to go after school”. (G28) 


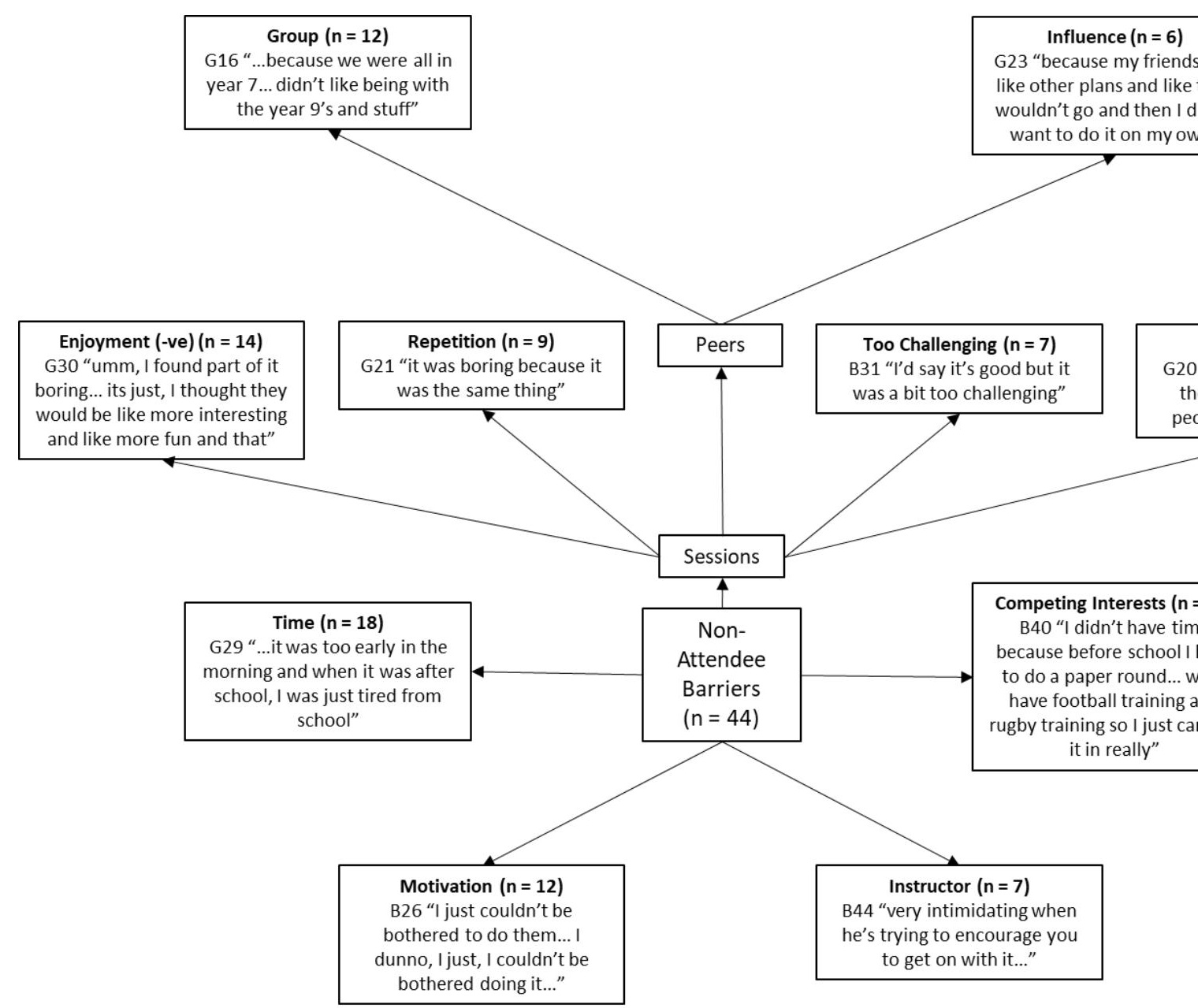

Figure 6.4 Non-attendee barriers - pen profile of perceptions of a 6-month high-intensity interval training ir negative 


\section{Discussion}

The aim of this study was to explore participant perceptions of the X4ACJ HIIT intervention. Data revealed that HIIT may be an enjoyable and engaging form of exercise providing certain factors are considered. The findings suggest that a range of facilitators and barriers to HIIT relating to session timing, exercise activities, group composition, instructor characteristics, exercise difficulty and exercise session protocol should be considered to maximise adherence.

The personal benefits of exercise emerged as the primary facilitator to participation in HIIT, particularly the perceived physiological improvements accrued via the intervention. Indeed, contrary to previous research, albeit in girls only ( $S$. Whitehead \& Biddle, 2008), the majority of the participants in the present study identified health benefits, such as fitness, rather than weight management, as the main physiological benefit:

"It improved fitness which helped with other sports like football... just like I felt like I could run more in football'. (B12)

Aligning with past literature (Mackintosh et al., 2011; McGuire, Neumark-Sztainer, \& Story, 2002; Weinberg et al., 2000), this is encouraging as it indicates that adolescents can conceptualise the association between exercise, health and fitness. However, for the non-attendee group, this awareness of the benefits of exercise did not translate into participation (Withall, Jago, \& Fox, 2011). This highlights that alternative forms of motivation are required to improve participation. For example, in line with the selfdetermination theory (Ryan \& Deci, 2000), although feedback from the health measures may facilitate greater participation for the extrinsically motivated (Proper, van der Beek, Hildebrandt, Twisk, \& Van, 2003; J. R. Whitehead \& Corbin, 1991), for the intrinsically motivated, factors concerning competence and enjoyment are rated higher as reasons for participation (Ryan, Frederick, Lepes, Rubio, \& Sheldon, 1997; Weinberg et al., 2000). Contrastingly, it could be postulated that more immediate motivations such as material rewards were required to increase participation, and although this study did aim to cater for this in a limited capacity, given that the prizes were mainly activity-based, these may not have appealed to the more sedentary nonattendees. Upon reflection, however, encouraging participants to attend an exercise intervention purely for material rewards may not be appropriate in that it somewhat 
defeats the object in terms of developing an appealing exercise intervention that can improve health and instil long-term behaviour change.

In accordance with previous studies that also focused on youths' perceptions of exercise (Allender, Cowburn, \& Foster, 2006; Dishman et al., 2005; Fairclough \& Stratton, 2005), certain aspects of the HIIT sessions themselves also influenced attendance, irrespective of attendance rates. Of intrigue, enjoyment, previously questioned in HIIT due to insufficient evidence (Logan et al., 2014), was identified as a primary facilitator for both groups. However, a lack of enjoyment also emerged as a dominating barrier within the non-attendee group. It could be postulated that such divergence in opinion from the attendees was related to the time at which they ceased to attend. Indeed, as put forward by Varey and Kahneman (1992), final experiences can bear a strong influence on a person's overall judgement. As such, given that the session content was progressively enhanced throughout the intervention, for those participants that only attended at the initial sessions, their judgement was based on possibly the least enjoyable sessions, whereas for the attendees, their judgement was based on the most enjoyable. Regardless, consistent with Mackintosh et al. (2011), Winn et al. (2017) and Logan et al. (2014) these findings further accentuate the need for exercise sessions to be enjoyable. Linked to enjoyment (Mandigo, Holt, Anderson, \& Sheppard, 2008), games within the sessions were also viewed as a key facilitator. Indeed, research that conducted games-based HIIT interventions in adolescents reported exceptionally high adherence rates (K. L. Weston et al., 2016). Furthermore, taking part in team games has been associated with numerous social benefits (Gray, Sproule, \& Wang, 2008), also identified as a key facilitator to exercise participation (Passer, 1981).

Whilst the challenging nature of HIIT was identified as a facilitator by some despite the exercise intensity being relative to individuals, particularly within the nonattendees group, some participants felt that the sessions were too challenging, representing a significant barrier to participation (Slater \& Tiggemann, 2010; S. Whitehead \& Biddle, 2008). In conjunction with a lack of enjoyment, such findings are perhaps also related to the dual-mode theory given that participants were required to exercise above their ventilatory threshold (Ekkekakis, 2003). As posited by Parfitt and Hughes (2009), cognitive displeasure and strong interoceptive cues as a result of high-intensity exercise (Ekkekakis, 2003) can result in a high negative peak in 
affective valence, which could subsequently deter future participation. However, given that a number of participants both enjoyed the sessions and relished the challenge, this theory may be limited, though, such contradictory findings may be explained by the acute nature of Ekkekakis' research compared to the long-term nature of the present study. Furthermore, the dual-mode theory was based primarily on adults whereas the present findings are children's perceptions. Alternatively, therefore, it could be postulated that the non-attendees were potentially less physically fit and able to complete the exercise and, therefore, less inclined to exercise and participate in the intervention (Boyle, Jones, \& Walters, 2008). Regardless, given these findings, it could be proposed that future research should carefully consider participant capabilities and the associated exercise difficulty within the sessions, facilitating personalisation via intra-exercise variability. For example, knee press-ups instead of full press-ups could be suggested as an alternative. Furthermore, although from the facilitators perspective the exercises were easily adaptable to individuals' relative intensity, perhaps greater attention should be taken to ensure that tailoring the exercise to the appropriate intensity is manageable for all participants. Failing to do so may limit the fidelity of the intervention as participants may not be able to achieve the required HR. Alternatively, it could also be postulated that HIIT is possibly too challenging for adolescents and that other modes of exercise should be pursued. This being said, although accentuating poor physical competence can negatively influence participation (Sallis et al., 2000), adolescents suggested the integration of abilityspecific groups alleviate issues relating to exercise difficulty:

"It could sort of be you have different sessions depending on what ability group you're in ... on a Monday it could be the higher and Tuesday it could be the lower...". (G11)

Adolescents' perceptions of the intervention instructor also determined intervention adherence. Research has shown that positive instructor role models can significantly increase youth's physical activity levels and subsequent intention to exercise (Eddolls, McNarry, Stratton, \& Mackintosh, 2016; Humbert et al., 2008; Olivares, CossioBolaños, Gomez-Campos, Almonacid-Fierro, \& Garcia-Rubio, 2015). Equally, however, it has been suggested that poor role models can be detrimental to exercise participation (Slater \& Tiggemann, 2010). Interestingly, a possible reason for the disparity between participants' perceptions is due to the instructor often having to discipline misbehaving participants. This may, therefore, have given the impression of 
a negative demeanour and favouritism towards certain groups (C. A. Williams \& Stevens, 2013) and consequently a negative perception. As such, this suggests that health practitioners should be aware of the prominent role instructors can have on future intervention success.

Analogous with Juvancic-Heltzel, Glickman and Barkley (2013) who found that increased exercise choice, participation and enjoyment improved performance, variation emerged as a key facilitator. Despite this, however, a greater number of participants felt that the variation, or lack thereof, in the sessions was a barrier, irrespective of group. It is possible that such discrepancies in opinion are related to inter-participant differences and, indeed, self-determination (Ryan \& Deci, 2000). For example, those that found the sessions repetitive participated because they were extrinsically motivated by the potential benefits, whereas, those that felt that the variety was adequate may have participated because they were intrinsically motivated by the inherent satisfaction (i.e., enjoyment) of the exercise (Frederick \& Ryan, 1993; Ryan et al., 1997). Therefore, whilst it is difficult to facilitate given the naturally repetitive nature of HIIT, it could be recommended that future research should focus on integrating a wider range of exercises or, as per the self-determination theory (Ryan $\&$ Deci, 2000), provide participants with some autonomy over the session design.

Despite offering opportunities to attend either before or after school, or indeed a variety of both, the time of the intervention sessions were identified as a major barrier to participation. This is somewhat surprising given that the intervention was developed utilising formative research which suggested that, for the majority for participants, delivering sessions outside of school hours was a viable option (Winn et al., 2017). As such, and in combination with the intervention school being unwilling to allocate time to the intervention during the school day, the intervention was implemented outside of school hours. As informed by formative research (Winn et al., 2017), whereby some participants indicated that they would prefer sessions before school and others suggesting that they would prefer sessions after school, both were offered to maximise participation. Nonetheless, ultimately participants either disliked waking up early to attend morning sessions, with some also stating that they would feel too tired at the end of school to go to the session: 
"It was too early in the morning and when it was after school, I was just tired from school”. (G29)

Several participants, especially within the non-attendee groups, also identified barriers such as competing interests, for example, extracurricular sports, jobs or other commitments (Winn et al., 2017). Consequently, given HIIT's previously demonstrated feasibility (Costigan, Eather, Plotnikoff, Taaffe, Pollock, et al., 2015), it could be suggested that future research should consider embedding a HIIT intervention within the school day. Indeed, previous research has successfully demonstrated that incorporating HIIT within a PE lesson can facilitate participation and elicit significant health improvements (K. L. Weston et al., 2016).

Perhaps due to the greater level of exposure given the time spent at the sessions, poor peer behaviour was established as a predominant barrier within the attendees group. These findings are in line with research that suggests that negative experiences during exercise with peers can adversely influence participation (Ries et al., 2008; Slater \& Tiggemann, 2010). Furthermore, in addition to its negative influence on participation, such poor behaviour may have also adversely affected the intervention's fidelity, an integral component of a trial's validity (Bellg et al., 2004; Horner, Rew, \& Torres, 2006; Taylor, Weston, \& Batterham, 2015), for example:

"We stopped all the time because there was people who were messing about, whereas we wanted to start the exercises off, but they kept on mucking about, so we never got to do what we should have done”. (B8)

Linked to poor behaviour, peer isolation was a technique utilised by the instructor to control adverse behaviour, a factor that negatively influenced the participants perceptions of the intervention (Knowles, Niven, \& Fawkner, 2011). Although such opinions are likely to be from the perspective of the participants who got removed, it is possible that such situations were also compounded by overcrowding during the sessions, a factor also viewed by the participants as a barrier. Indeed, when a greater number of participants attended a single session, the probability of detecting adverse behaviour was increased and, subsequently, increased the likelihood of peer isolation. Consequently, given that positive social experiences can foster adherence (Kelleher et al., 2017), to ameliorate such barriers, it could be proposed that future interventions should consider alternative disciplinary techniques (Blatchford, Bassett, \& Brown, 
2011) and also ensure groups remain with a manageable size in order to allow for greater control.

Whilst many of barriers to the intervention cited were somewhat unsurprising, the identification of sprinting/running exercises as a barrier was unexpected given that these were specifically incorporated based on formative research (Winn et al., 2017). These findings therefore question the reliability of adolescents' preconceived perceptions of exercise and traditional qualitative formative research methods. It is therefore suggested that future research should align "taster" sessions with traditional formative research methods, to obtain more informed perceptions. Specifically, the integration of iterative user-engaged intervention development could lead to more enjoyable and sustainable interventions.

Although the present study has various strengths, certain limitations should be acknowledged. It is important to consider the potential influence of self-selection bias on the present findings due to the voluntary nature of the intervention (Ganguli, Lytle, Reynolds, \& Dodge, 1998). Whilst the present study engaged with participants that had signed up to the intervention but did not regularly attend, it could be argued that these participants were still biased, given they still contemplated participation. An additional limitation is that peer pressure may have influenced focus-group discussions and therefore outcomes. In order to conform with perceived socially-desirable responses, participants may have modelled their answers accordingly (Paulhus \& Vazire, 2005). Participants may also have felt pressured not to oppose, or alternatively, to agree with dominant members of the group (Leung \& Savithiri, 2009). It could also be postulated that some participants may have found focus-groups an intimidating environment and could have therefore withheld their opinions. Whilst the author conducting the focus-groups had attended the exercise sessions and built a rapport with some of the participants, it is noteworthy that participants may not have felt comfortable being critical of the intervention. Nonetheless, the authors felt the experience and rapport would have extracted more insightful information. An additional limitation to the study was the lack of interviews with other people involved with the intervention, such as the Commando Joe's instructor. Eliciting the opinions of the X4ACJ project from a facilitator's standpoint may have provided an alternative, yet valuable, insight into some of the themes extracted from the attendee and nonattendee participants. 


\section{Conclusion}

This study was the first to explore adolescents' perceptions of HIIT and elicit their views on the barriers and facilitators to continued adherence. These findings, in combination with evidence advocating HIIT as a means to improve health in adolescents, provide support for the efficacy of HIIT as a population-based health intervention strategy. Findings suggest that researchers should employ a fun and varied games-based intervention within physical education lessons led by a well-qualified and approachable instructor. Sessions should also be well planned and consider the possible variation in ability between participants. Additionally, in conjunction with running or sprinting-based activities, repetition within and between sessions should be avoided. 


\section{Thesis study map}

\begin{tabular}{|c|c|c|}
\hline Study & & Outcomes \\
\hline $\begin{array}{l}\text { The association } \\
\text { between physical } \\
\text { activity, fitness and } \\
\text { body size and their } \\
\text { effect on mental } \\
\text { well-being and } \\
\text { quality of life in } \\
\text { children and } \\
\text { adolescents }\end{array}$ & Key Findings & $\begin{array}{l}\text { To investigate the relationships between } \\
\text { VPA, BMI and cardiorespiratory fitness on } \\
\text { symptoms of depression, and their } \\
\text { subsequent direct and indirect effects on QoL } \\
\text { in children and adolescents } \\
\text { Vigorous physical activity, cardiorespiratory } \\
\text { fitness and BMI are associated, both directly } \\
\text { and indirectly, with mental well-being and } \\
\text { QoL in children and adolescents. }\end{array}$ \\
\hline $\begin{array}{l}\text { The effect of high- } \\
\text { intensity interval } \\
\text { training on mental } \\
\text { well-being and } \\
\text { quality of life in } \\
\text { adolescents: the } \\
\text { eXercise for Asthma } \\
\text { with Commando } \\
\text { Joe's }{ }^{\circledR} \text { (X4ACJ) trial }\end{array}$ & Key Findings & $\begin{array}{l}\text { To assess the short-term and longer-term } \\
\text { effects of a school-based HIIT intervention } \\
\text { on body mass index, cardiorespiratory } \\
\text { fitness, physical activity levels, depression, } \\
\text { anxiety and QoL in adolescents. } \\
\text { HIIT does not significantly improve BMI, } \\
\text { cardiorespiratory fitness, physical activity, } \\
\text { depression, anxiety or QoL in adolescents. }\end{array}$ \\
\hline $\begin{array}{l}\text { Exploring adolescent } \\
\text { perceptions of a } \\
\text { high-intensity } \\
\text { interval training } \\
\text { intervention: } \\
\text { evaluating the } \\
\text { eXercise for Asthma } \\
\text { with Commando } \\
\text { Joe's }{ }^{\circledR}(\mathrm{X} 4 \mathrm{ACJ}) \text { trial }\end{array}$ & Key Findings & $\begin{array}{l}\text { To explore adolescents' perceptions of the } \\
\text { eXercise for Asthma with Commando Joe's }{ }^{\circledR} \\
\text { (X4ACJ) HIIT intervention and to elicit their } \\
\text { views on the barriers and facilitators to initial } \\
\text { and continued participation. } \\
\text { Incorporating a fun and varied games-based } \\
\text { HIIT intervention into physical education } \\
\text { lessons led by a well-qualified and } \\
\text { approachable instructor, whilst avoiding } \\
\text { running or sprinting-based activities, has the } \\
\text { potential to engage adolescents and could } \\
\text { enhance participation in HIIT. }\end{array}$ \\
\hline
\end{tabular}




\section{Chapter 7 - Synthesis}

\section{General Summary}

This thesis presents three studies with the overarching aim of assessing the effects of high-intensity interval training (HIIT) on mental well-being and quality of life (QoL) in adolescents. This thesis includes a systematic review, which explored previous adolescent HIIT interventions with the aim of establishing the optimal HIIT protocol. Following the identification of an optimal protocol based on physiological measures, to use the recommended guidelines within a psychological and QoL intervention, associations between physiological and psychological health were established. Having determined that physiological health was significantly associated with psychological health and QoL, using the information from the systematic review in conjunction with additional formative research (Winn et al., 2017), the second component of this thesis was to implement and measure the effectiveness of the developed protocol. The third and final study presented a post-intervention evaluation of the trial from the participants' perspectives.

\section{Summary of individual study findings}

Whilst there is a wealth of literature that has identified the beneficial effects of HIIT on physiological health (Rosenkranz et al., 2012; Tjønna et al., 2009; K. L. Weston et al., 2016), there remains a paucity of research exploring the effects of HIIT on mental well-being and QoL. Furthermore, the two studies (Costigan et al., 2016; Shepherd et al., 2015) that previously explored HIIT and mental health reported contradictory findings, though this could be attributed to the different exercise protocols. Given this dearth of literature, conducting a systematic review and establishing an optimal protocol based on psychological literature was not possible and, therefore, an alternative approach to forming a suitable exercise protocol was required. Subsequently, to elucidate the most effective HIIT protocol a systematic review was conducted that examined HIIT as a means of influencing key physiological health parameters (Eddolls et al., 2017; Chapter 3). In accord with this aim, thirteen studies were evaluated, providing evidence that HIIT can significantly improve certain health parameters in children and adolescents. Furthermore, optimal HIIT protocol guidelines were established, with findings suggesting running-based sessions, at an intensity of 90\% maximum heart rate (HR)/100-130\% maximal aerobic velocity, 2-3 times a week 
and with a minimum intervention duration lasting 7 weeks, elicits the greatest improvements to health.

Since the findings of the systematic review were based on physiological measures, to use the recommended HIIT protocol guidelines within a psychological and QoL intervention, associations between the subcomponents of physiological and psychological health were required. Consequently, Study 1 aimed to investigate the direct and indirect relationships between physiological health, psychological health and QoL, using structural equation modelling (SEM). Within this cross-sectional study, 576 adolescents' physical activity, body mass index (BMI), cardiorespiratory fitness, depression and QoL were assessed. The results indicated that vigorous physical activity (VPA), cardiorespiratory fitness and BMI were associated, both directly and indirectly, with depression and QoL. Furthermore, higher levels of VPA were associated with a greater cardiorespiratory fitness, lower BMI and healthier state of mental well-being. As such, it could be suggested that improving physiological health via HIIT, the structured form of VPA, may elicit improvements to psychological health and QoL.

Following protocol guidelines identified within the systematic review (Eddolls et al., 2017; Chapter 3) in combination with formative research (Winn et al., 2017), completed as part of the overall eXercise for asthma with Commando Joe's (X4ACJ) project, an intervention was developed and implemented in order to establish the effect of HIIT on mental well-being and QoL. Study 2 examined the effect of the X4ACJ HIIT intervention on BMI, cardiorespiratory fitness, physical activity, anxiety, depression and QoL in adolescents. Participants were invited to 3 x 30-minute sessions per week for 6 months. Within the sessions, participants completed multiple bouts of maximal exercise with the aim of eliciting a HR greater than $90 \%$ age predicted maximum HR. Bouts lasted between 10-30 seconds, followed by an equal rest. Highintensity interval training did not significantly improve BMI, cardiorespiratory fitness, physical activity, anxiety, depression or QoL in the intervention group. Consequently, whilst a number of limitations within the study may have confounded the results, these findings suggest that HIIT does not benefit certain physical health outcomes or mental well-being or QoL. Nevertheless, no detrimental effects to mental health or QoL were detected as a result of the HIIT intervention. 
Given the lack of intervention effect on adolescents' mental health or QoL, the importance of post-intervention evaluation was especially paramount in order to elicit an understanding of the possible intervention shortfalls (Hoffmann et al., 2014). Therefore, the aim of Study 3 was to explore adolescents' perceptions of the X4ACJ HIIT intervention and the barriers and facilitators to participation. Participants were interviewed via semi-structured focus-groups, with data from two groups (attendee and non-attendee) transcribed verbatim and thematically analysed deductively, with key emergent themes represented using pen profiles. The findings indicated that HIIT is a viable and enjoyable form of exercise for adolescents, however, to maximise participation, factors related to session timing, exercise activities, group composition, instructor and exercise progression and protocol should be accounted for. Specifically, data suggested that embedding a HIIT intervention within the school day may improve adherence. Furthermore, sessions should be split into manageable group sizes based on year groups, with the exercise led by a well-qualified, motivational and approachable instructor. Prior to conducting the intervention sessions, lessons should be well planned and varied to avoid repetition and should allow for progression or regression within exercises. Whilst running and sprinting exercises should be avoided, competitive invasion games were deemed the most enjoyable.

\section{General discussion}

In line with the aims stated in Chapter 1, the present thesis developed, conducted and evaluated a HIIT intervention aimed at enhancing adolescents' mental health and QoL. Considering the mixed findings within this thesis, in this section, issues related to the intervention design will be discussed, in addition to the differences between Study 1 and Study 2. Furthermore, potential reasons for the variance in findings within all the studies will be covered.

\section{Intervention design}

The X4ACJ HIIT intervention was developed following the guidance of formative research (Winn et al., 2017) and a systematic review (Eddolls et al., 2017; Chapter 3) while also abiding by the restrictions placed by the participating school. As part of the six-month trial, adolescents were invited to attend $3 \times$ 30-minute exercise sessions per week held on alternative weekdays, either before or after the school day. The exercise sessions, led by a Commando Joe's ${ }^{\circledR}$ instructor, partly replicated previous HIIT 
interventions (Baquet et al., 2010; Boddy et al., 2010; Racil et al., 2016; Sperlich et al., 2011), with participants asked to complete multiple bouts of various exercises, each lasting between 10-30 seconds, followed by an equal period of rest. During the exercise phase, to comply with previously outlined standards (Hood et al., 2011; Little et al., 2011), the exercises were designed to induce a HR exceeding 90\% maximum HR.

Despite a paucity of comparative research, the intervention within Study $\mathbf{2}$ draws some parallels with previous intervention designs. Similar to Costigan et al. (2016), Study 2 followed comparable exercise and rest interval timings, with the exercises completed during the intervention also consisting of a combination of cardiorespiratory and body weight resistance exercises. Furthermore, akin with Study 2, Costigan and colleagues (2016) conducted their intervention within a school with adolescents. Despite stark differences regarding session and intervention duration, the methodological similarities regarding the intervention design between both studies may perhaps explain the analogous findings. In contrast, however, Shepherd et al. (2015) reported significant improvements to psychological health as a result of HIIT. It should be noted, however, that these findings were a result of a laboratory-based mechanicallybraked spinning bike intervention, in which extraneous and independent variables could be closely controlled, thus lowering the ecological validity of the findings. Moreover, it could also be postulated that, despite comparable session durations and exercise interval timings to Study 2, the disparity in results is perhaps due to Shepherd et al. (2015) focusing exclusively on inactive adults. Consequently, future research should perhaps consider the relationship between baseline fitness and the potential magnitude of change over the course of a HIIT intervention.

Although the methodological differences between Study 2 and Shepherd et al. (2015) may partly explain the contrasting findings, several other restrictions related to the intervention design may also have confounded the results. A key factor that likely effected the statistical outcomes of Study 2 was the low sample size, a consequence of the barriers highlighted within Study 3. Indeed, many of those invited to attend the X4ACJ HIIT intervention were either unable to participate due to competing interests or did not want to relinquish their spare time. Furthermore, despite Buchan, Ollis, Thomas, Malina and Baker (2012) recommending physical education lessons as the ideal setting, integrating the entire HIIT intervention within physical education lessons 
within school day was unfeasible. Indeed, similar to issues faced in previous research (Taylor, 2014), the limited proportion of the academic timetable allocated to physical education, conducting 3 x 30-minute sessions within those lessons was not possible. Moreover, similar to the issues described in Buchan et al. (2012), the participating school were unwilling to allocate a portion of other parts of the school timetable specifically for the intervention. Additionally, although previous interventions have used physical education lessons to conduct HIIT (Costigan et al., 2016; K. L. Weston et al., 2016), the intervention school in the present thesis stated the reason that they were not willing to incorporate the intervention within the school day was that the specific exercise activities did not align with the national curriculum. Therefore, to ameliorate issues relating to competing interests or participant aversion to relinquishing their own time, it is recommended that future studies consider the requirements of the national curriculum prior to developing prospective school-based exercise interventions to enable their implementation within physical education lessons. This is especially relevant given the findings within Study 3 and previous research (Donnelly et al., 2016), in which increased physical activity and exercise has been associated with improved academic performance.

Another factor that possibly limited the design of the HIIT intervention was the use of the systematic review (Eddolls et al., 2017; Chapter 3) to form the intervention protocol. Despite Study 1 supporting the use of the physiological findings in the systematic review (Eddolls et al., 2017; Chapter 3) for use within a psychological intervention, it is highly possible that such protocol guidelines are not appropriate to induce improvements to mental health. Consequently, to consolidate findings related to the effect of HIIT on mental health and QoL, impending research should further explore a range of differing protocols. Despite designing the exercise activities using information collected from future participants during formative work (Winn et al., 2017), once participants were reassessed as part of the post-intervention evaluation (Study 3), many of the pre-conceived ideas had altered. For example, the finding of the inclusion of sprinting/running exercises being a barrier within Study $\mathbf{3}$ was somewhat surprising given that the formative review identified it as enjoyable (Winn et al., 2017). Consequently, although widely used across research (Boddy, Knowles, et al., 2012; Mackintosh et al., 2011), these findings bring into question the accuracy of traditional qualitative formative research in exercise. Indeed, when comparing the 
formative research (Winn et al., 2017) and the post-intervention evaluation (Study 3) it appears that adolescents' perceptions can ultimately change once they have had the opportunity to experience said exercise. Therefore, prior to future formative research, to obtain a more informed response, researchers should endeavour to provide "taster" sessions as part of a pilot study.

\section{Effects of VPA and HIIT on mental health and QoL}

The present thesis provides contrasting perspectives as to the effect of high-intensity physical activity or exercise on mental health and QoL. Within Study 1, higher levels of VPA, mediated by BMI, were shown to be indicative of fewer symptoms of depression and greater QoL. Conversely, Study 2 reported that HIIT did not elicit any significant improvements in symptoms of depression or QoL, with significant changes in anxiety attributed to factors outside of the intervention. Since HIIT is a structured form of VPA, the variance in findings between Study $\mathbf{1}$ and Study 2 is intriguing. Specifically, given that the physical activity accrued via HIIT is essentially VPA, the results between Studies $\mathbf{2}$ and $\mathbf{3}$ should be similar.

It could be postulated that the primary reason for the variance between Study $\mathbf{1}$ and Study 2 is that Study 2 did not significantly improve BMI or cardiorespiratory fitness. Within Study 1, increased VPA was shown to only improve depression and QoL indirectly, mediated by BMI and cardiorespiratory fitness, respectively. Subsequently, it could be suggested that without eliciting significant improvements in BMI or cardiorespiratory fitness, HIIT was unable to significantly improve depression, and subsequently QoL. This is further supported by previous research in which physiological improvements attributed to physical activity and exercise, for example, improved body composition (Drenowatz et al., 2016; Gutin et al., 2002), can elicit improvements in self-image and self-esteem (C. E. Ross, 1994), which have both been associated with mental health (Lubans et al., 2016; Markowitz et al., 2008). This suggests that interventions should be multifaceted and aim to improve both physiological and psychological health rather than exclusively concentrating on just one component of health.

Given the relationship between VPA and HIIT, it could also be theorised that the difference in findings between Study $\mathbf{1}$ and Study $\mathbf{2}$ is a result of sample size issues. Indeed, within the intervention group in Study 2, there is scope to suggest that the lack 
of significance, at least in part, may be due to the relatively small sample size and thus decreased statistical power. An additional reason for the variance in findings is perhaps that since Study 2 is cross-sectional in design, causality cannot be inferred and therefore the study is unable to detect the direction of a relationship. Accordingly, it could be proposed that lower symptoms of depression and good QoL are indicative of higher levels of VPA, rather than the other way around. Nevertheless, it has been suggested that if using appropriate and robust statistical analysis, such as SEM, observational trials (Study 1) may better reflect the wider population compared to randomised controlled trials performed in a homogenous sub-group such as in Study 2 (Faraoni \& Schaefer, 2016).

In summary, since there are constraints to both cross-sectional (Study 1) and randomised control trials (Study 2), neither study types should be considered in isolation. Rather, researchers should form their interpretations of results based on methodological rigour (Berger et al., 2012). Therefore, considering the limitations of both studies, it should be assumed that neither study is conclusive. Subsequently, further research is needed to determine the effects of VPA and HIIT on mental health and QoL.

\section{Thesis strengths}

A major strength of this thesis was the documentation of the development, implementation and evaluation of the X4ACJ project. Specifically, this thesis provided a supporting evidence that VPA, the non-structured form of HIIT, could ameliorate mental health and QoL (Study 1), tested the effectiveness of implementing a HIIT intervention as a means of improving mental health and QoL (Study 2) and evaluated the HIIT intervention in terms of participant perceptions (Study 3). Furthermore, this thesis explored a relatively novel area of research, with only a limited number of studies published to date (Costigan et al., 2016; Shepherd et al., 2015). An additional strength that is worth noting is that the intervention was school-based and not laboratory-based. This demonstrates that HIIT can be implemented within schools, with limited equipment. Indeed, although more recent research has moved towards implementing HIIT within a real-world setting (Costigan et al., 2016; K. L. Weston et al., 2016), much of the research to date remains laboratory-based (Baquet et al., 2010; 
Rosenkranz et al., 2012; Tjønna et al., 2009), therefore limiting the ecological validity of their findings.

\section{Thesis limitations}

There are several limitations within this thesis, primarily related to study design. A confounding factor that may have may have introduced bias was the non-blinding of groups. Given that the leading researchers were responsible for both the data collection and intervention implementation, conducting a blind assessment was not possible. However, since the control group participants were not informed that there was an additional group receiving an exercise intervention, it is unlikely such bias would have impaired the results.

A further limitation to this thesis relates to the fidelity to the HIIT protocol, or the degree to which the intervention followed the principles of HIIT. In accord with the key principals of HIIT and in line with the thresholds set out (Hood et al., 2011; Little et al., 2011), the target intensity of the intervention was set at $>90 \%$ HR maximum. However, although still above the threshold for physiological gain (Baquet et al., 2003), HR data suggested that the intervention elicited an average of $\sim 80 \% \mathrm{HR}$ maximum over the exercise sessions. Although, it is pertinent to note that, whilst both the warm-up and cool-down periods were removed, this average includes between exercise rest periods, with mean maximum HR exceeding 90\%. It could also be postulated that the short bout duration and quicker recovery of adolescents compared to adults (Falk \& Dotan, 2006) may have influenced participant HR outcomes. Specifically, participants may have returned to nearer baseline values between each bout, thereby requiring a greater subsequent intensity or duration to achieve a HR maximum greater than $90 \%$. Regardless, participants exceeded the threshold of $>90 \%$ HR maximum $24 \%$ of the time, which, when considering they were at rest for half of the sessions, suggests that the intervention should be considered HIIT. An additional limitation to this study related to HR validity was the reliability of the HR measurement equipment. Although the HR data within the present thesis was in line with previous HIIT research, in many cases, there was a "drop-out" in data, whereby, for short periods, the HR monitors would fail to pick up participants HRs, possibly related to being dislodged due to the vigorous exercise, reducing the overall average. Furthermore, perhaps unavoidable when working with adolescents, poor participant 
behaviour during the sessions occasionally resulted in elongated rest periods to allow the facilitator to discipline. Indeed, a key barrier to participation that was identified within Study 3 was poor behaviour by peers. Considering the limitations related to intervention fidelity and HR reliability, future studies should consider more accurate methods to determine exercise intensity during each exercise session and indeed bouts, which may also allow a dose-response relationship to be elucidated.

An additional limitation of this thesis in relation to work presented in Study $\mathbf{2}$ was the small sample size. Although it was the intention to deliver the X4ACJ HIIT intervention to a large sample, this was not achieved; possibly due to the various barriers featured in Study 3. Indeed, despite the intervention largely being informed by formative research that included participants from the intervention school (Winn et al., 2017), adherence was poor. Therefore, considering the relatively small sample size and subsequent decreased statistical power, it is possible to speculate that this may have confounded any potential significant findings. Accordingly, the findings in Study 2 may not provide an accurate reflection of the effect of HIIT on mental health and QoL. From a statistical analysis standpoint, it could be suggested that future research should consider evaluating practical/clinical significance using the minimum clinically important difference (Batterham \& Hopkins, 2006). However, in terms of participant recruitment and retention, from the findings within Study 3, the primary reason for the lack of attendance was that the sessions took place either prior to or after school. Many participants articulated that they had other commitments, ranging from school sport to being young carers, or that they just did not want to get out of bed early to participate or would rather play with their friends after school:

"I purely couldn't be bothered... it's too early in the morning... and I just couldn't be bothered to go after school”. (G28)

Consequently, to ameliorate this, the leading recommendation would be to ensure that the intervention sessions were embedded within the school day, similar to previous HIIT interventions (Boddy et al., 2010; Costigan, Eather, Plotnikoff, Taaffe, Pollock, et al., 2015; K. L. Weston et al., 2016).

Finally, with respect to the mental health and QoL instruments utilised within this thesis, in line with their instructions, all were measured using self-reporting techniques. In addition to issues related to social desirability (Paulhus \& Vazire, 2005), 
this type of measurement is limited as it relies on participants perceptions of health to be equal, which may not have been the case. Furthermore, it could be postulated that respondent interest may have confounded the results of this thesis. Indeed, given the number of participants completing the measures at any one time, the ability to control and focus every participant's attention exclusively on the questionnaire was unfeasible. Consequently, participants may have answered their questionnaire without due care, potentially effecting the overall findings. Despite this, however, each questionnaire had been previously validated for use within child and adolescent populations and scored adequately with regard to Cronbach's Alpha (Cronbach, 1951).

\section{Implications for future research}

From the work completed in this thesis, in order to further research in HIIT and mental health, there are several recommendations that should be taken into consideration prior to undertaking any future studies. Although some qualitative formative research was conducted for this thesis (Winn et al., 2017), it was limited. As such, before prescribing future HIIT interventions, more comprehensive formative research is warranted. Researchers should consider implementing short pilot studies prior to conducting any long-term large-scale studies, not only to test the effectiveness of any newly designed protocols, but also to gain an understanding of participant's perceptions of the intervention prior to conducting any main trial. Additionally, as highlighted within Chapter 2, there is limited information regarding an "optimal" exercise protocol in terms of exercise dose within adolescent mental health research. As such, more comprehensive formative research may provide greater clarity as to the best frequency, intensity, time and type of exercise to induce improvements to mental health.

Since research to date has only used relatively small samples, larger, more suitablypowered trials are required to provide a more comprehensive assessment of the effects of HIIT on mental well-being and QoL. Consequently, researchers should aim to maximise participant recruitment and ensure participant retention remains high. Based on the findings within Study $\mathbf{3}$, it is recommended that future research integrates prospective HIIT interventions within the school day. It would therefore be beneficial for future interventions to align the exercise activities incorporated within the intervention with the national curriculum. Collaborating with physical education teachers to ensure that future interventions fulfil the national curriculum requirements 
is therefore recommended. Additionally, in line with the findings in Study 3, future research should ensure that interventions employ a fun and varied games-based intervention sessions led by a well-qualified and approachable instructor. Sessions should also be well planned and consider the possible variation in ability between participants and avoid running or sprinting-based activities and repetition within and between sessions.

Given the findings within Study 1, which suggested that VPA was only indirectly associated with depression and QoL, mediated by BMI and cardiorespiratory fitness, future HIIT interventions aiming to improve mental health should remain multifaceted. It is recommended that prospective interventions should primarily target increasing VPA, improving cardiorespiratory fitness and reducing BMI, with the secondary target being to improve mental health and QoL. As such, researchers should monitor participants' VPA levels, cardiorespiratory fitness and BMI throughout HIIT interventions. Indeed, given the postulated minimum intervention duration threshold (Eddolls et al., 2017), to improve mental health outcomes, it may be necessary to amend the session content if the HIIT intervention does not deliver positive physiological results after 7 weeks. Additionally, future research should aim to address issues relating to physical activity accrued outside of interventions. Accordingly, future studies should report objectively measured physical activity levels to assess the potential ramifications of a "compensation effect" that may negate the beneficial effects of HIIT.

\section{Overall conclusion}

Work within this thesis indicates that physiological health is associated with psychological health and QoL, both directly and indirectly. Additionally, increased VPA was correlated with improvements in BMI, symptoms of depression, cardiorespiratory fitness and QoL. Regardless of this, for anxiety, depression and QoL, such benefits did not translate when structured VPA was applied using a HIIT intervention. However, given that the prescribed HIIT intervention was designed using guidelines based on physiological outcomes, despite the association between physiological health and psychological health and QoL, such a protocol may have been unsuitable. Nonetheless, no detrimental effects were noted because of HIIT, therefore this thesis suggests that it should be considered a feasible and time-efficient form of exercise that can improve physical health. Furthermore, given that previous research 
has demonstrated that HIIT can significantly improve mental well-being in adults, taking into consideration the present thesis' limitations and taking forward the implications for future research, there is scope to suggest that HIIT has the potential to improve mental well-being and QoL in adolescents. 


\section{References}

Abarca-Gómez, L., Abdeen, Z. A., Hamid, Z. A., Abu-Rmeileh, N. M., AcostaCazares, B., Acuin, C., ... Ezzati, M. (2017). Worldwide trends in body-mass index, underweight, overweight, and obesity from 1975 to 2016: a pooled analysis of 2416 population-based measurement studies in 128.9 million children, adolescents, and adults. The Lancet.

Abildgaard, J. S., Saksvik, P. Ø., \& Nielsen, K. (2016). How to measure the intervention process? An assessment of qualitative and quantitative approaches to data collection in the process evaluation of organizational interventions. Frontiers in Psychology, 7, 1380.

Ajilore, O., Haroon, E., Kumaran, S., Darwin, C., Binesh, N., Mintz, J., ... Kumar, A. (2007). Measurement of brain metabolites in patients with type 2 diabetes and major depression using proton magnetic resonance spectroscopy. Neuropsychopharmacology, 32, 1224-1231.

Aldwin, C. M. (1991). Does age affect the stress and coping process? Implications of age differences in perceived control. Journal of Gerontology, 46(4), 174-180.

Allender, S., Cowburn, G., \& Foster, C. (2006). Understanding participation in sport and physical activity among children and adults: a review of qualitative studies. Health Education Research, 21(6), 826-835.

American Diabetes Association. (2000). Type 2 diabetes in children and adolescents. Pediatrics, 105(3), 671-680.

American Heart Association. (1997). Obesity and heart disease. Circulation, 96(9), $3248-3250$.

Andersen, J. R., Natvig, G. K., Aadland, E., Moe, V. F., Kolotkin, R. L., Anderssen, S. A., \& Resaland, G. K. (2017). Associations between health-related quality of life, cardiorespiratory fitness, muscle strength, physical activity and waist circumference in 10-year-old children: the ASK study. Quality of Life Research, 26(12), 3421-3428.

Andersen, L. B., Harro, M., Sardinha, L. B., Froberg, K., Ekelund, U., Brage, S., \& Anderssen, S. A. (2006). Physical activity and clustered cardiovascular risk in children: a cross-sectional study (The European Youth Heart Study). The Lancet, 368, 299-304.

Anderson, L., Oldridge, N., Thompson, D. R., Zwisler, A. D., Rees, K., Martin, N., \& Taylor, R. S. (2016). Exercise-based cardiac rehabilitation for coronary heart disease: Cochrane systematic review and meta-analysis. Journal of the American College of Cardiology, 67(1), 1-12.

Andrews, G., \& Slade, T. (2001). Interpreting scores on the Kessler Psychological Distress Scale (K10). Australian and New Zealand Journal of Public Health, 25(6), 494-497.

Angold, A., Costello, E. J., Messer, S. C., Pickles, A., Winder, F., \& Silver, D. (1995). The development of a short questionnaire for use in epidemiological studies of depression in children and adolescents. International Journal of 
Methods in Psychiatric Research, 5, 237-249.

Bailey, R. C., Olson, J., Pepper, S. L., Porszasz, J., Barstow, T. J., \& Cooper, D. M. (1995). The level and tempo of children's physical activities: an observational study. Medicine and Science in Sports Exercise, 27, 1033-1041.

Baker, D. (2011). Recent trends in high-intensity aerobic training for field sports. Professional Strength \& Conditioning, 27, 4-8.

Bandura, A. (1997). Self-efficacy: The exercise of control. New York, NY: W H Freeman and Company.

Baquet, G., Berthoin, S., Dupont, G., Blondel, N., Fabre, C., \& van Praagh, E. (2002). Effects of high intensity intermittent training on peak VO2 in prepubertal children. International Journal of Sports Medicine, 23, 439-444.

Baquet, G., Berthoin, S., Gerbeaux, M., \& van Praagh, E. (2001). High-intensity aerobic training during a 10 week one-hour physical education cycle: effects on physical fitness of adolescents aged 11 to 16. International Journal of Sports Medicine, 22(4), 295-300.

Baquet, G., Gamelin, F., Mucci, P., Thévenet, D., van Praagh, E., \& Berthoin, S. (2010). Continuous vs. interval aerobic training in 8- to 11-year-old children. Journal of Strength and Conditioning Research, 24(5), 1381-1388.

Baquet, G., Guinhouya, C., Dupont, G., Nourry, C., \& Berthoin, S. (2004). Effects of a short-term interval training program on physical fitness in prepubertal children. Journal of Strength And Conditioning Research, 18(4), 708-713.

Baquet, G., van Praagh, E., \& Berthoin, S. (2003). Endurance training and aerobic fitness in young people. Sports Medicine, 33(15), 1127-1143.

Barlow, S. E., \& Expert Committee. (2007). Expert committee recommendations regarding the prevention, assessment, and treatment of child and adolescent overweight and obesity: summary report. Pediatrics, 120(Suppl 4), S164-192.

Bastard, J., Maachi, M., Lagathu, C., Kim, M. J., Caron, M., Vidal, H., ... Feve, B. (2006). Recent advances in the relationship between obesity, inflammation, and insulin resistance. European Cytokine Network, 17(1), 4-12.

Batterham, A. M., \& Hopkins, W. G. (2006). Making meaningful inferences about magnitudes. International Journal of Sports Physiology and Performance, 1, $50-57$.

Baxter-Jones, A. D. G., Eisenmann, J. C., \& Sherar, L. B. (2005). Controlling for Maturation in Pediatric Exercise Science. Pediatric Exercise Science, 17(1), 1830 .

Beck, A. T., Epstein, N., Brown, G., \& Steer, R. A. (1988). An inventory for measuring clinical anxiety: psychometric properties. Journal of Consulting and Clinical Psychology, 56(6), 893-897.

Beck, A. T., Steer, R. A., Ball, R., \& Ranieri, W. F. (1996). Comparison of Beck Depression Inventories-IA and-II in psychiatric outpatients. Journal of Personality Assessment, 67(3), 588-597.

Beck, A. T., Ward, C. H., Mendelson, M., Mock, J., \& Erbaugh, J. (1961). Inventory 
for measuring depression. Archives of General Psychiatry, 4, 561-571.

Beckham, E. E. (2000). Depression. In A. E. Kazdin (Ed.), Encyclopedia of Psychology. American Psychological Association and Oxford University Press.

Belanoff, J. K., Kalehzan, M., Sund, B., Fleming Ficek, S. K., \& Schatzberg, A. F. (2001). Cortisol activity and cognitive changes in psychotic major depression. American Journal of Psychiatry, 158(10), 1612-1616.

Bellg, A. J., Resnick, B., Minicucci, D. S., Ogedegbe, G., Ernst, D., Borrelli, B., ... Czajkowski, S. (2004). Enhancing treatment fidelity in health behavior change studies: best practices and recommendations from the NIH Behavior Change Consortium. Health Psychology, 23(5), 443-451.

Berger, M. L., Dreyer, N., Anderson, F., Towse, A., Sedrakyan, A., \& Normand, S. L. (2012). Prospective observational studies to assess comparative effectiveness: the ISPOR good research practices task force report. Value in Health, 15(2), 217-230.

Biddle, S. J. H., \& Asare, M. (2011). Physical activity and mental health in children and adolescents: a review of reviews. British Journal of Sports Medicine, 45, 886-895.

Biddle, S. J. H., Gorely, T., \& Stensel, D. J. (2004). Health-enhancing physical activity and sedentary behaviour in children and adolescents. Journal of Sports Sciences, 22(8), 679-701.

Biddle, S. J. H., Markland, D., Gilbourne, D., Chatzisarantis, N. L. D., \& Sparkes, A. C. (2001). Research methods in sport and exercise psychology: quantitative and qualitative issues. Journal of Sports Sciences, 19(10), 777-809.

Billat, L. (2001). Interval training for performance: a scientific and empirical practice. Sports Medicine, 31(1), 13-31.

Binagwaho, A., Stulac, S., \& Beardslee, W. R. (2012). Validating the Center for Epidemiological Studies Depression Scale for Children in Rwanda. Journal of the American Academy of Child \& Adolescent Psychiatry, 51(12), 1284-1292.

Bize, R., Johnson, J. A., \& Plotnikoff, R. C. (2007). Physical activity level and health-related quality of life in the general adult population: a systematic review. Preventive Medicine, 45(6), 401-415.

Bjelland, I., Dahl, A. A., Haug, T. T., \& Neckelmann, D. (2002). The validity of the hospital anxiety and depression scale an updated literature review. Journal of Psychosomatic Research, 52, 69-77.

Blair, S. N., Clark, D. G., Cureton, K. J., \& Powell, K. E. (1989). Exercise and fitness in childhood: implications for a lifetime of health. In C. V Gisolfi \& D. R. Lamb (Eds.), Perspectives in Exercise Science and Sports Medicine. New York, NY: McGraw-Hill.

Blatchford, P., Bassett, P., \& Brown, P. (2011). Examining the effect of class size on classroom engagement and teacher-pupil interaction: differences in relation to pupil prior attainment and primary vs. secondary schools. Learning and Instruction, 21, 715-730. 
Blumenthal, J. A., Williams, R. S., Needels, T. L., \& Wallace, A. G. (1982).

Psychological changes accompany aerobic exercise in healthy middle-aged adults. Psychosomatic Medicine, 44(6), 529-536.

Boddy, L. M., Knowles, Z. R., Davies, I. G., Warburton, G. L., Mackintosh, K. A., Houghton, L., \& Fairclough, S. J. (2012). Using formative research to develop the healthy eating component of the CHANGE ! school-based curriculum intervention. BMC Public Health, 12, 710.

Boddy, L. M., Stratton, G., Hackett, A. F., \& George, K. P. (2010). The effectiveness of a 'short, sharp, shock' high intensity exercise intervention in 11- and 12-yearold Liverpool schoolgirls. Archives of Exercise in Health and Disease, 44, 1925.

Boddy, L. M., Thomas, N. E., Fairclough, S. J., Tolfrey, K., Brophy, S., Rees, A., ... Stratton, G. (2012). ROC generated thresholds for field-assessed aerobic fitness related to body size and cardiometabolic risk in schoolchildren. PLOS ONE, $7(9), 8-12$.

Bonhauser, M., Fernandez, G., Püschel, K., Yañez, F., Montero, J., Thompson, B., \& Coronado, G. (2005). Improving physical fitness and emotional well-being in adolescents of low socioeconomic status in Chile: results of a school-based controlled trial. Health Promotion International, 20(2), 113-122.

Boreham, C. A., Paliczka, V. J., \& Nichols, A. K. (1990). A comparison of the PWC170 and 20-MST tests of aerobic fitness in adolescent schoolchildren. The Journal of Sports Medicine and Physical Fitness, 30(1), 19-23.

Bostic, T. J., McGartland, D., \& Hood, M. (2000). A validation of the subjective vitality scale using structural equation modeling. Social Indicators Research, 52(3), 313-324.

Boyle, S. E., Jones, G. L., \& Walters, S. J. (2008). Physical activity among adolescents and barriers to delivering physical education in Cornwall and Lancashire, UK: a qualitative study of heads of PE and heads of schools. $B M C$ Public Health, 9, 279.

Braithwaite, I., Stewart, A. W., Hancox, R. J., Murphy, R., Wall, C. R., Beasley, R., ... ISAAC Phase Three Study Group. (2017). Body mass index and vigorous physical activity in children and adolescents: an international cross-sectional study. Acta Paediatrica, 106(8), 1323-1330.

Braun, V., \& Clarke, V. (2006). Using thematic analysis in psychology. Qualitative Research in Psychology, 3(2), 77-101.

Brodersen, N. H., Steptoe, A., Boniface, D. R., \& Wardle, J. (2007). Trends in physical activity and sedentary behaviour in adolescence: ethnic and socioeconomic differences. British Journal of Sports Medicine , 41, 140-144.

Brooks, S. J., \& Kutcher, S. (2001). Diagnosis and measurement of adolescent depression: a review of commonly utilized instruments. Journal of Child and Adolescent Psychopharmacology, 11(4), 341-376.

Brown, H. E., Pearson, N., Braithwaite, R. E., Brown, W. J., \& Biddle, S. J. H. (2013). Physical activity interventions and depression in children and 
adolescents: a systematic review and meta-analysis. Sports Medicine, 43(3), 195-206.

Brown, J. D., \& Lawton, M. (1986). Stress and well-being in adolescence: The moderating role of physical exercise. Journal of Human Stress, 12(3), 125-131.

Brown, S. W., Welsh, M. C., Labbé, E. E., Vitulli, W. F., \& Kulkarni, P. (1992). Aerobic exercise in the psychological treatment of adolescents. Perceptual and Motor Skills, 74, 555-560.

Buchan, D. S., Ollis, S., Thomas, N. E., Buchanan, N., Cooper, S.-M., Malina, R. M., \& Baker, J. S. (2011). Physical activity interventions: effects of duration and intensity. Scandinavian Journal of Medicine \& Science in Sports, 21(6), 341350 .

Buchan, D. S., Ollis, S., Thomas, N. E., Malina, R. M., \& Baker, J. S. (2012). School-based physical activity interventions: challenges and pitfalls. Child: Care, Health and Development, 38(1), 1-2.

Buchan, D. S., Ollis, S., Young, J. D., Cooper, S., Shield, J. P. H., \& Baker, J. S. (2013). High intensity interval running enhances measures of physical fitness but not metabolic measures of cardiovascular disease risk in healthy adolescents. BMC Public Health, 13(1), 498.

Buchheit, M., \& Laursen, P. B. (2013). High-intensity interval training, solutions to the programming puzzle: Part I: Cardiopulmonary emphasis. Sports Medicine, 43(5), 313-338.

Butte, N. F., Wong, W. W., Adolph, A. L., Puyau, M. R., Vohra, F. A., \& Zakeri, I. F. (2010). Validation of cross-sectional time series and multivariate adaptive regression splines models for the prediction of energy expenditure in children and adolescents using doubly labeled water. Journal of Nutrition, 140(8), 15161523.

Calle, E. E., \& Thun, M. J. (2004). Obesity and cancer. Oncogene, 23, 6365-6378.

Caspersen, C. J., Powell, K. E., \& Christenson, G. M. (1985). Physical activity, exercise, and physical fitness: definitions and distinctions for health-related research. Public Health Reports, 100(2), 126-131.

Cassilhas, R. C., Antunes, H. K. M., Tufik, S., \& de Mello, M. T. (2010). Mood, anxiety, and serum IGF-1 in elderly men given 24 weeks of high resistance exercise. Perceptual and Motor Skills, 110(1), 265-276.

Catellier, D. J., Hannan, P. J., Murray, D. M., Addy, C. L., Conway, T. L., Yang, S., \& Rice, J. C. (2005). Imputation of missing data when measuring physical activity by accelerometry. Medicine and Science in Sports and Exercise, 37, S555-S562.

Christensen, E. H., Hedman, R., \& Saltin, B. (1960). Intermittent and continuous running. Acta Physiologica Scandinavica, 50, 269-286.

Christian, D., Todd, C., Hill, R., Rance, J., Mackintosh, K. A., Stratton, G., \& Brophy, S. (2016). Active children through incentive vouchers - evaluation (ACTIVE): a mixed-method feasibility study. BMC Public Health, 16(1), 890. 
Clarke, S. A., \& Eiser, C. (2004). The measurement of health-related quality of life (QOL) in paediatric clinical trials: a systematic review. Health and Quality of Life Outcomes, 2(1), 66.

Clarke, V., \& Braun, V. (2015). Thematic analysis. In E. Lyons \& A. Coyle (Eds.), Analysing Qualitative Data in Psychology (pp. 84-103). London, UK: Sage Publications.

Clennin, M. N., Payne, J. P. W., Rienzi, E. G., Lavie, C. J., Blair, S. N., Pate, R. R., $\&$ Sui, X. (2015). Association between cardiorespiratory fitness and healthrelated quality of life among patients at risk for cardiovascular disease in Uruguay. PLoS ONE, 10(4), e0123989.

Cohen, A. J., Brauer, M., Burnett, R., Anderson, H. R., Frostad, J., Estep, K., ... Forouzanfar, M. H. (2017). Estimates and 25-year trends of the global burden of disease attributable to ambient air pollution: an analysis of data from the Global Burden of Diseases Study 2015. The Lancet, 389(10082), 1907-1918.

Cohen, J. (1988). Statistical Power Analysis for the Behavioral Sciences. New York, NY: Routledge Academic.

Cohen, J. (1992). Quantitative methods in psychology. Psychological Bulletin, 112(1), 155-159.

Collishaw, S., Maughan, B., Natarajan, L., \& Pickles, A. (2010). Trends in adolescent emotional problems in England: a comparison of two national cohorts twenty years apart. The Journal of Child Psychology and Psychiatry, $51(8), 885-894$.

Cornelissen, V. A., \& Fagard, R. H. (2005). Effects of endurance training on blood pressure, blood pressure-regulating mechanisms, and cardiovascular risk factors. Hypertension, 46(4), 667-675.

Costello, A. J., Dulcan, M. K., \& Kalas, R. (1985). Age Differences in the Reliability of the Psychiatric Interview of the Child. Child Development, 56(1), 265-275.

Costigan, S. A., Eather, N., Plotnikoff, R. C., Hillman, C. H., \& Lubans, D. R. (2016). High-intensity interval training on cognitive and mental health in adolescents. Medicine and Science in Sports and Exercise, 48(10), 1985-1993.

Costigan, S. A., Eather, N., Plotnikoff, R. C., Taaffe, D. R., \& Lubans, D. R. (2015). High-intensity interval training for improving health-related fitness in adolescents: a systematic review and meta-analysis. British Journal of Sports Medicine, 49(19), 1253-1261.

Costigan, S. A., Eather, N., Plotnikoff, R. C., Taaffe, D. R., Pollock, E., Kennedy, S. G., \& Lubans, D. R. (2015). Preliminary efficacy and feasibility of embedding high intensity interval training into the school day: a pilot randomized controlled trial. Preventive Medicine Reports, 2, 973-979.

Costigan, S. A., Ridgers, N. D., Eather, N., Plotnikoff, R. C., Harris, N., \& Lubans, D. R. (2017). Exploring the impact of high intensity interval training on adolescents' objectively measured physical activity: findings from a randomized controlled trial. Journal of Sports Sciences, 36(10), 1087-1094.

Coventry, P. A., Bower, P., Keyworth, C., Kenning, C., Knopp, J., Garrett, C., ... 
Dickens, C. (2013). The effect of complex interventions on depression and anxiety in chronic obstructive pulmonary disease: systematic review and metaanalysis. PLoS ONE, 8(4), e60532.

Craft, L. L. (2005). Exercise and clinical depression: examining two psychological mechanisms. Psychology of Sport and Exercise, 6(2), 151-171.

Craft, L. L., \& Perna, F. M. (2004). The benefits of exercise for the clinically depressed. The Primary Care Companion to The Journal of Clinical Psychiatry, 6(3), 104-111.

Cronbach, L. J. (1951). Coefficient alpha and the internal structure of tests. Psychometrika, 16(3), 297-334.

Cui, W., \& Zack, M. M. (2013). Trends in health-related quality of life among adolescents in the United States, 2001 - 2010. Preventing Chronic Disease, 10, E111.

Cummins, R. A. (1997). Self-rated quality of life scales for people with an intellectual disability: a review. Journal of Applied Research in Intellectual Disabilities, 10(3), 199-216.

Cummins, R. A., Lau, A. L. D., \& Stokes, M. (2004). HRQOL and subjective wellbeing: noncomplementary forms of outcome measurement. Expert Review of Pharmacoeconomics \& Outcomes Research, 4, 413-420.

Curtis, L. (2012). Unit Costs of Health \& Social Care 2012. Canterbury.

Cusin, C., Yang, H., Yeung, A., \& Fava, M. (2010). Handbook of clinical rating scales and assessment in psychiatry and mental health. In L. Baer \& M. A. Blais (Eds.), Handbook of clinical rating scales and assessment in psychiatry and mental health (Vol. 121, pp. 487-488). Humana Press.

Da Costa, D., Rippen, N., Dritsa, M., \& Ring, A. (2003). Self-reported leisure-time physical activity during pregnancy and relationship to psychological well-being. Journal of Psychosomatic Obstetrics and Gynecology, 24(2), 111-119.

De Bourdeaudhuij, I., Van Cauwenberghe, E., Spittaels, H., Oppert, J. M., Rostami, C., Brug, J., ... Maes, L. (2011). School-based interventions promoting both physical activity and healthy eating in Europe: a systematic review within the HOPE project. Obesity Reviews, 12(3), 205-216.

De Civita, M., Regier, D., Alamgir, A. H., Anis, A. H., FitzGerald, M. J., \& Marra, C. A. (2005). Evaluating health-related quality-of-life studies in paediatric populations: some conceptual, methodological and developmental considerations and recent applications. Pharmacoeconomics, 23(7), 659-685.

de Wit, M., \& Hajos, T. (2013). Health related quality of life. In M. D. Gellman \& J. R. Turner (Eds.), Encyclopedia of Behavioral Medicine. New York, NY: Springer.

Department of Health. (2011). Start active, stay active: a report on physical activity from the four home countries' Chief Medical Officer. London, UK.

Derogatis, L. R. (1992). SCL-90-R, administration, scoring and procedures manualII for the R(evised) version and other instruments of the Psychopathology 
Rating Scale Series. Towson, MD: Clinical Psychometric Research.

Dias, K. A., Ingul, C. B., Tjønna, A. E., Keating, S. E., Gomersall, S. R., Follestad, T., ... Coombes, J. S. (2017). Effect of high-intensity interval training on fitness, fat mass and cardiometabolic biomarkers in children with obesity: a randomised controlled trial. Sports Medicine, 48(3), 733-746.

Diener, E., Emmons, R. A., Larsen, R. J., \& Griffin, S. (1985). The Satisfaction With Life Scale. Journal of Personality Assessment, 49(1), 71-75.

Diener, E., \& Suh, E. (1997). Measuring quality of life: economic, social, and subjective indicators. Social Indicators Research, 40, 189-216.

DiLorenzo, T. M., Bargman, E. P., Stucky-Ropp, R., Brassington, G. S., Frensch, P. A., \& LaFontaine, T. (1999). Long-term effects of aerobic exercise on psychological outcomes. Preventive Medicine: An International Journal Devoted to Practice and Theory, 28(1), 75-85.

Dilorio, C., Hockenberry-Eaton, M., Maibach, E., \& Rivero, T. (1994). Focus groups: an interview method for nursing research. The Journal of Neuroscience Nursing, 26(3), 175-180.

Dishman, R. K., Motl, R. W., Saunders, R., Felton, G., Ward, D. S., Dowda, M., \& Pate, R. R. (2005). Enjoyment mediates effects of a school-based physicalactivity intervention. Medicine and Science in Sports and Exercise, 37(3), 478487.

Donahue, K. E., Mielenz, T. J., Sloane, P. D., Callahan, L. F., \& Devellis, R. F. (2006). Identifying supports and barriers to physical activity in patients at risk for diabetes. Preventing Chronic Disease, 3(4), A119.

Donges, C. E., Duffield, R., \& Drinkwater, E. J. (2010). Effects of resistance or aerobic exercise training on interleukin-6, C-reactive protein, and body composition. Medicine and Science in Sports and Exercise, 42(2), 304-313.

Donnelly, J. E., Hillman, C. H., Castelli, D. M., Etnier, J. L., Lee, S., Tomporowski, P., ... Szabo-Reed, A. N. (2016). Physical activity, fitness, cognitive function, and academic achievement in children: a systematic review. Medicine and Science in Sports and Exercise, 48(6), 1197-1222.

Donovan, G. O., Blazevich, A. J., Boreham, C., Cooper, A. R., Ekelund, U., Fox, K. R., ... Stamatakis, E. (2010). The ABC of physical activity for health: a consensus statement from the British Association of Sport and Exercise Sciences. Journal of Sports Sciences, 28(6), 573-591.

Drenowatz, C., Prasad, V. K., Hand, G. A., Shook, R. P., \& Blair, S. N. (2016). Effects of moderate and vigorous physical activity on fitness and body composition. Journal of Behavioral Medicine, 39(4), 624-632.

Drotar, D. (2004). Validating measures of pediatric health status, functional status, and health-related quality of life: key methodological challenges and strategies. Ambulatory Pediatrics, 4(4), 358-364.

Dumith, S. C., Gigante, D. P., Domingues, M. R., \& Kohl, H. W. I. I. I. (2011). Physical activity change during adolescence: a systematic review and a pooled analysis. International Journal of Epidemiology, 40(3), 685-698. 
Dwyer, J., Allison, K. R., Goldenberg, E. R., Fein, A. J., Yoshida, K. K., \& Boutilier, M. A. (2006). Adolescent girls' perceived barriers to participation in physical activity. Adolescence, 41(161), 75-89.

Eather, N., Morgan, P. J., \& Lubans, D. R. (2016). Effects of exercise on mental health outcomes in adolescents: findings from the CrossFit ${ }^{\mathrm{TM}}$ teens randomized controlled trial. Psychology of Sport \& Exercise, 26, 14-23.

Eddolls, W. T. B., McNarry, M. A., Lester, L., Winn, C. O. N., Stratton, G., \& Mackintosh, K. A. (2018). The association between physical activity, fitness and body mass index on mental well-being and quality of life in adolescents. Quality of Life Research.

Eddolls, W. T. B., McNarry, M. A., Stratton, G., \& Mackintosh, K. A. (2016). Parental influences on children's physical self-perceptions, body composition and physical activity levels. The Lancet, $388, \mathrm{~S} 45$.

Eddolls, W. T. B., McNarry, M. A., Stratton, G., Winn, C. O. N., \& Mackintosh, K. A. (2017). High-intensity interval training interventions in children and adolescents: a systematic review. Sports Medicine, 47(11), 2363-2374.

Eiser, C., \& Jenney, M. (2007). Measuring quality of life. Archives of Disease in Childhood, 92(4), 348-350.

Eiser, C., Mohay, H., \& Morse, R. (2000). The measurement of quality of life in young children. Child: Care, Health and Development, 26(5), 401-414.

Eiser, C., \& Morse, R. (2001). The measurement of quality of life in children: past and future perspectives. Developmental and Behavioral Pediatrics, 22(4), 248256.

Ekelund, U., Steene-Johannessen, J., Brown, W. J., Wang Fagerland, M., Owen, N., Powell, K. E., ... Lee, I.-M. (2016). Physical activity attenuates the detrimental association of sitting time with mortality: a harmonised meta-analysis of data from more than one million men and women. The Lancet, 388(1051), 13021310 .

Ekkekakis, P. (2003). Pleasure and displeasure from the body: perspectives from exercise. Cognition and Emotion, 17(2), 213-239.

Esposito, M., Gallai, B., Roccella, M., Marotta, R., Lavano, F., Lavano, S. M., ... Carotenuto, M. (2014). Anxiety and depression levels in prepubertal obese children: a case-control study. Neuropsychiatric Disease and Treatment, 10, 1897-1902.

Evenson, K. R., Catellier, D. J., Gill, K., Ondrak, K. S., \& McMurray, R. G. (2008). Calibration of two objective measures of physical activity for children. Journal of Sports Sciences, 26(14), 1557-1565.

Fairclough, S. J., \& Stratton, G. (2005). Physical activity levels in middle and high school physical education: a review. Pediatric Exercise Science, 17(3), 217.

Falk, B., \& Dotan, R. (2006). Child-adult differences in the recovery from highintensity exercise. Exercise and Sport Sciences Reviews, 34(3), 107-112.

Faraoni, D., \& Schaefer, S. T. (2016). Randomized controlled trials vs. observational 
studies: why not just live together? BMC Anesthesiology, 16(1), 1-4.

Farina, N., Rusted, J., \& Tabet, N. (2014). The effect of exercise interventions on cognitive outcome in Alzheimer's disease: a systematic review. International Psychogeriatrics, 26(1), 9-18.

Faulkner, G., \& Biddle, S. J. H. (2001). Exercise and mental health: it's just not psychology! Journal of Sports Sciences, 19(6), 433-444.

Fendrich, M., Weissman, M. M., \& Warner, V. (1990). Screening for depressive disorder in children and adolescents: validating the Centre for Epidemiologic Studies Depression Scale for Children. American Journal of Epidemiology, 131(3), 538-551.

Fernandez-Fernandez, J., Zimek, R., Wiewelhove, T., \& Ferrauti, A. (2012). Highintensity interval training vs. repeated-sprint training in tennis. Journal of Strength and Conditioning Research, 26(1), 53-62.

Ferrans, C. E., Zerwic, J. J., Wilbur, J. E., \& Larson, J. L. (2005). Conceptual model of health-related quality of life. Journal of Nursing Scholarship, 37(4), 336342.

Ferrari, A. J., Charlson, F. J., Norman, R. E., Patten, S. B., Freedman, G., Murray, C. J. L., ... Whiteford, H. A. (2013). Burden of depressive disorders by country, sex, age, and year: findings from the Global Burden of Disease Study 2010. PLoS Medicine, 10(11), e1001547.

Field, A. (2013). Discovering statistics using IBM SPSS statistics (4th ed.). London, UK: SAGE.

Fisak Jr, B. J., Richard, D., \& Mann, A. (2011). The prevention of child and adolescent anxiety: a meta-analytic review. Prevention Science, 12, 255-268.

Folkins, C. H., \& Sime, W. E. (1981). Physical fitness training and mental health. American Psychologist, 36(4), 373-389.

Fox, K. R. (1999). The influence of physical activity on mental well-being. Public Health Nutrition, 2(3a), 411-418.

Fox, K. R., Cooper, A., \& McKenna, J. (2004). The school and promotion of children's health-enhancing physical activity: perspectives from the United Kingdom. Journal of School Health, 23(2001), 338-358.

Francois, M. E., \& Little, J. P. (2015). Effectiveness and safety of high-intensity interval training in patients with type 2 diabetes. Diabetes Spectrum, 28(1), 3944.

Franks, P. W., Hanson, R. L., Knowler, W. C., Sievers, M. L., Bennett, P. H., \& Looker, H. C. (2010). Childhood obesity, other cardiovascular risk factors and premature death. The New England Journal of Medicine, 362, 485-493.

Frederick, C. M., \& Ryan, R. M. (1993). Differences in motivation for sport and exercise and their relationships with participation and mental health. Journal of Sport Behaviour, 16(3), 125-145.

Freedman, D. S., Mei, Z., Srinivasan, S. R., Berenson, G. S., \& Dietz, W. H. (2007). Cardiovascular risk factors and excess adiposity among overweight children and 
adolescents: the Bogalusa Heart Study. The Journal of Pediatrics, 150(1), 1217.

Funtikova, A. N., Navarro, E., Bawaked, R. A., Fíto, M., \& Schröder, H. (2015). Impact of diet on cardiometabolic health in children and adolescents. Nutrition Journal, 14, 118.

Füssenich, L. M., Boddy, L. M., Green, D. J., Graves, L. E. F., Foweather, L., Dagger, R. M., \& McWhannell, N. (2016). Physical activity guidelines and cardiovascular risk in children: a cross sectional analysis to determine whether 60 minutes is enough. BMC Public Health, 16(67).

Ganguli, M., Lytle, M. E., Reynolds, M. D., \& Dodge, H. H. (1998). Random versus volunteer selection for a community-based study. Journal of Gerontology, 53(1), 39-46.

Garber, C. E., Blissmer, B., Deschenes, M. R., Franklin, B. A., Lamonte, M. J., Lee, I.-M., ... Swain, D. P. (2011). Quantity and quality of exercise for developing and maintaining neuromotor fitness in apparently healthy adults: guidance for prescribing exercise. Medicine and Science in Sports and Exercise, 43(7), 13341359.

Gauvin, L., \& Rejeski, W. J. (1993). The exercise-induced feeling inventory: development and initial validation. Journal of Sport and Exercise Psychology, $15,403-423$.

Gelenberg, A. J., Freeman, M. P., Markowitz, J. C., Rosenbaum, J. F., Thase, M. E., Trivedi, M. H., \& Van Rhoads, R. S. (2010). Practice guideline for the treatment of patients with major depressive disorder (3rd ed.). Arlington, VA: American Psychiatric Association.

Gerber, M., Brand, S., Herrmann, C., Colledge, F., Holsboer-Trachsler, E., \& Pühse, U. (2014). Increased objectively assessed vigorous-intensity exercise is associated with reduced stress, increased mental health and good objective and subjective sleep in young adults. Physiology and Behavior, 135, 17-24.

Gibala, M. J. (2007). High-intensity interval training: a time-efficient strategy for health promotion? Current Sports Medicine Reports, 6(4), 211-213.

Gibala, M. J., Little, J. P., Macdonald, M. J., \& Hawley, J. A. (2012). Physiological adaptations to low-volume, high-intensity interval training in health and disease. The Journal of Physiology, 590(5), 1077-1084.

Gibala, M. J., \& McGee, S. L. (2008). Metabolic adaptations to short-term highintensity interval training: a little pain for a lot of gain? Exercise and Sport Sciences Reviews, 36, 58-63.

Gibson, F. (2007). Conducting focus groups with strategies for success. Journal of Research in Nursing, 12(5), 473-483.

Gill, D. L., Hammond, C. C., Reifsteck, E. J., Jehu, C. M., Williams, R. A., Adams, M. M., ... Shang, Y.-T. (2013). Physical activity and quality of life. Journal of Preventive Medicine and Public Health, 46, S28-S34.

Gillis, L. J., Kennedy, L. C., Gillis, A. M., \& Bar-Or, O. (2002). Relationship between juvenile obesity, dietary energy and fat intake and physical activity. 
International Journal of Obesity, 26(4), 458-463.

Giraldi, T. (2017). Unhappiness, Sadness and "Depression": Antidepressants and the Mental Disorder Epidemic. Basingstoke, UK: Palgrave Macmillan.

Gladstone, T. R. G., Beardslee, W. R., \& O'Connor, E. E. (2011). The prevention of adolescent depression. Psychriatric Clinics of North America, 34(1), 35-52.

Goldfield, G. S., Henderson, K., Buchholz, A., Obeid, N., Nguyen, H., \& Flament, M. F. (2011). Physical activity and psychological adjustment in adolescents. Journal of Physical Activity and Health, 8(2), 157-163.

Gomersall, S. R., Rowlands, A. V, English, C., Maher, C., \& Olds, T. S. (2013). The ActivityStat hypothesis. Sports Medicine, 43, 135-149.

Graham, R., Kremer, J., \& Wheeler, G. (2008). Physical exercise and psychological well-being among people with chronic illness and disability: a grounded approach. Journal of Health Psychology, 13(4), 447-458.

Gray, S., Sproule, J., \& Wang, C. K. J. (2008). Pupils' perceptions of and experiences in team invasion games: a case study of a Scottish secondary school and its three feeder primary schools. European Physical Education Review, 14(2), 179-201.

Green, H., McGinnity, A., Meltzer, H., Ford, T., \& Goodman, R. (2004). Mental health of children and young people in Great Britain.

Guiraud, T., Nigam, A., Gremeaux, V., Meyer, P., Juneau, M., \& Bosquet, L. (2012). High-intensity interval training in cardiac rehabilitation. Sports Medicine, 42(7), 587-605.

Gutin, B., Barbeau, P., Owens, S., Lemmon, C. R., Bauman, M., Allison, J., ... Litaker, M. S. (2002). Effects of exercise intensity on cardiovascular fitness, total body composition, and visceral adiposity of obese adolescents. The American Journal of Clinical Nutrition, 75, 818-826.

Hagger, M. S., Chatzisarantis, N., \& Biddle, S. J. H. (2001). The influence of selfefficacy and past behaviour on the physical activity intentions of young people. Journal of Sports Sciences, 19(9), 711-725.

Häkkinen, A., Rinne, M., Vasankari, T., Santtila, M., Häkkinen, K., \& Kyröläinen, H. (2010). Association of physical fitness with health-related quality of life in Finnish young men. Health and Quality of Life Outcomes, 8(15).

Hamilton, M. (1960). A rating scale for depression. Journal of Neurology, Neurosurgery, and Psychiatry, 23, 56-62.

Hamlin, M. J., Fraser, M., Lizamore, C. A., Draper, N., Shearman, J. P., \& Kimber, N. E. (2014). Measurement of cardiorespiratory fitness in children from two commonly used field tests after accounting for body fatness and maturity. Journal of Human Kinetics, 40(1), 83-92.

Hansen, H. S., Froberg, K., Hyldebrandt, N., \& Nielsen, J. R. (1991). A controlled study of eight months of physical training and reduction of blood pressure in children: the Odense schoolchild study. BMJ, 303(6804), 682-685.

Haskell, W. L., Lee, I.-M., Pate, R. R., Powell, K. E., \& Blair, S. N. (2007). Physical 
activity and public health: updated recommendation for adults from the American College of Sports Medicine and the American Heart Association. Circulation, 116(9), 1081-1093.

Hassmén, P., Koivula, N., \& Uutela, A. (2000). Physical exercise and psychological well-being: a population study in Finland. Preventive Medicine, 30(1), 17-25.

Heitzler, C., Lytle, L., Erickson, D., Sirard, J., Barr-Anderson, D., \& Story, M. (2011). Physical activity and sedentary activity patterns among children and adolescents: a latent class analysis approach. Journal of Physical Activity and Health, 8(4), 457-467.

Helgadóttir, B., Forsell, Y., \& Ekblom, Ö. (2015). Physical activity patterns of people affected by depressive and anxiety disorders as measured by accelerometers: a cross-sectional study. PLoS ONE, 10(1), 1-10.

Helseth, S., \& Misvær, N. (2010). Adolescents' perceptions of quality of life: what it is and what matters. Journal of Clinical Nursing, 19(9-10), 1454-1461.

Herr, N. R., Williams Jr, J. W., Benjamin, S., \& McDuffie, J. (2014). Does this patient have generalized anxiety or panic disorder? The Rational Clinical Examination systematic review. Journal of the American Medical Association, 312(1), 78-84.

Higgins, J. P. T., Sterne, J. A. C., Savović, J., Page, M. J., Hróbjartsson, A., Boutron, I., ... Eldridge, S. (2016). A revised tool for assessing risk of bias in randomized trials. In J. Chandler, J. McKenzie, I. Boutron, \& V. Welch (Eds.), Cochrane Methods. Cochrane Database of Systematic Reviews.

Hill, D. M., Carvell, S., Matthews, N., Weston, N. J. V, \& Thelwell, R. R. C. (2017). Exploring choking experiences in elite sport: the role of self-presentation. Psychology of Sport and Exercise, 33, 141-149.

Hill, M. (1997). Participatory research with children. Child and Family Social Work, 2, 171-183.

Hilyer, J. C., Wilson, D. G., Dillon, C., Caro, L., Jenkins, C., Spencer, W. A., ... Booker, W. (1982). Physical fitness training and counseling as treatment for youthful offenders. Journal of Counseling Psychology, 29(3), 292-303.

Hoelscher, D. M., Feldman, H. A., Johnson, C. C., Lytle, L. A., Osganian, S. K., Parcel, G. S., ... Nader, P. R. (2004). School-based health education programs can be maintained over time: Results from the CATCH Institutionalization study. Preventive Medicine, 38(5), 594-606.

Hoffmann, T. C., Glasziou, P. P., Boutron, I., Milne, R., Perera, R., Moher, D., ... Michie, S. (2014). Better reporting of interventions: template for intervention description and replication (TIDieR) checklist and guide. BMJ, 348.

Holsboer, F. (2000). The corticosteroid receptor hypothesis of depression. Neuropsychopharmacology, 23(5), 477-501.

Hood, M. S., Little, J. P., Tarnopolsky, M. A., Myslik, F., \& Gibala, M. J. (2011). Low-volume interval training improves muscle oxidative capacity in sedentary adults. Medicine and Science in Sports and Exercise, 43(10), 1849-1856. 
Horner, S., Rew, L., \& Torres, R. (2006). Enhancing intervention fidelity: a means of strengthening study impact. Journal for Specialists in Pediatric Nursing, 11(2), $80-89$.

Houben-van Herten, M., Bai, G., Hafkamp, E., Landgraf, J. M., \& Raat, H. (2015). Determinants of health-related quality of life in school-aged children: a general population study in the Netherlands. PLoS ONE, 10(5), e0125083.

Huber, M., Knottnerus, J. A., Green, L., van der Horst, H., Jadad, A. R., Kromhout, D., ... Smid, H. (2011). How should we define health? BMJ, 343(7817), 1-3.

Hughes, C., Barnes, S., Barnes, C., Defina, L., Nakonezy, P., \& Emslie, G. (2013). Depresses Adolescents treated with Exercise (DATE): a pilot randomized controlled trial to test feasibility and establish perliminary effect sizes. Mental Health and Physical Activity, 6(2).

Humbert, M. L., Chad, K. E., Bruner, M. W., Spink, K. S., Muhajarine, N., Anderson, K. D., ... Gryba, C. R. (2008). Using a naturalistic ecological approach to examine the factors influencing youth physical activity across grades 7 to 12. Health Education \& Behaviour, 35, 158-173.

Hunter, J. E., \& Schmidt, F. L. (2004). Methods of meta-analysis: correcting error and bias in research findings (2nd ed.). Thousand Oaks, CA: SAGE.

Hussey, J., Bell, C., \& Gormley, J. (2007). The measurement of physical activity in children. Physical Therapy Reviews, 12(1), 52-58.

Hwang, C. L., Wu, Y. T., \& Chou, C. H. (2011). Effect of aerobic interval training on exercise capacity and metabolic risk factors in people with cardiometabolic disorders: a meta-analysis. Journal of Cardiopulmonary Rehabilitation and Prevention, 31(6), 378-385.

Janssen, I., Katzmarzyk, P. T., Boyce, W. F., Vereecken, C., Mulvihill, C., Roberts, C., ... Pickett, W. (2005). Comparison of overweight and obesity prevalence in school-aged youth from 34 countries and their relationships with physical activity and dietary patterns. Obesity Reviews, 6(2), 123-132.

Janssen, I., \& LeBlanc, A. G. (2010). Systematic review of the health benefits of physical activity and fitness in school-aged children and youth. The International Journal of Behavioral Nutrition and Physical Activity, 7, 40.

Janssens, L., Gorter, J. W., Ketelaar, M., Kramer, W. L. M., \& Holtslag, H. R. (2008). Health-related quality-of-life measures for long-term follow-up in children after major trauma. Quality of Life Research, 17, 701-713.

Jeong, Y.-J., Hong, S.-C., Lee, M. S., Park, M.-C., Kim, Y.-K., \& Suh, C.-M. (2005). Dance movement therapy improves emotional responses and modulates neurohormones in adolescents with mild depression. International Journal of Neuroscience, 115(12), 1711-1720.

Johnson, C. C., Murray, D. M., Elder, J. P., Jobe, J. B., Dunn, A. L., Kubik, M., ... Schachter, K. (2008). Depressive symptoms and physical activity in adolescent girls. Medicine and Science in Sports and Exercise, 40(5), 818-826.

Julian, L. J. (2011). Measures of anxiety: State-Trait Anxiety Inventory (STAI), Beck Anxiety Inventory (BAI), and Hospital Anxiety and Depression Scale- 
Anxiety (HADS-A). Arthritis Care \& Research, 63(Suppl 11), S467-472.

Juvancic-Heltzel, J. A., Glickman, E. L., \& Barkley, J. E. (2013). The effect of variety on physical activity: a cross-sectional study. Journal of Strength and Conditioning Research, 27(1), 244-251.

Kearns, B., Ara, R., Young, T., \& Relton, C. (2013). Association between body mass index and health-related quality of life, and the impact of self-reported longterm conditions - cross-sectional study from the south Yorkshire cohort dataset. BMC Public Health, 13, 1009.

Kelleher, E., Davoren, M. P., Harrington, J. M., Shiely, F., Perry, I. J., \& McHugh, S. M. (2017). Barriers and facilitators to initial and continued attendance at community-based lifestyle programmes among families of overweight and obese children: a systematic review. Obesity Reviews, 18(2), 183-194.

Kenchaiah, S., Sesso, H. D., \& Gaziano, J. M. (2009). Body mass index and vigorous physical activity and the risk of heart failure among men. Circulation, 119(1), 44-52.

Kennedy, C., Kools, S., \& Krueger, R. (2001). Methodological considerations in children's focus groups. Nursing Research, 50(3), 184-187.

Kesäniemi, A., Riddoch, C. J., Reeder, R., Blair, S. N., \& Sørensen, T. I. A. (2010). Advancing the future of physical activity guidelines. International Journal of Behavioural Nutrition and Physical Activity, 7(41), 1-14.

Kessler, H. S., Sisson, S. B., \& Short, K. R. (2012). The potential for high-intensity interval training to reduce cardiometabolic disease risk. Sports Medicine, 42(6), 489-509.

Kessler, R. C., \& Üstün, T. B. (2004). Historical development and present status of the schedule for affective disorders and schizophrenia for school-age children (K-SADS). International Journal of Methods in Psychiatric Research, 13(2), 93-121.

Knowles, A. M., Niven, A., \& Fawkner, S. (2011). A qualitative examination of factors related to the decrease in physical activity behavior in adolescent girls during the transition from primary to secondary school. Journal of Physical Activity and Health, 8, 1084-1091.

Koot, H. M. (2001). The study of quality of life: concepts and methods. In J. L. Wallander \& H. M. Koot (Eds.), Quality of life in child and adolescent illness. Concepts, methods and findings. Sussex: Brunner-Routledge.

Kopelman, P. G. (2000). Obesity as a medical problem. Nature, 404(6778), 635-643.

Korkiakangas, E. E., Alahuhta, M. A., \& Laitinen, J. H. (2009). Barriers to regular exercise among adults at high risk or diagnosed with type 2 diabetes: a systematic review. Health Promotion International, 24(4), 416-427.

Koscik, R. L., Douglas, J. A., Zaremba, K., Rock, M. J., Splaingard, M. L., Laxova, A., \& Farrell, P. M. (2005). Quality of life of children with cystic fibrosis. The Journal of Pediatrics, 147(3), S64-S68.

Kosher, H., Jiang, X., Ben-Arieh, A., \& Huebner, S. (2014). Advances in children's 
rights and children's well-being measurement: implications for school psychologists. School Psychology Quarterly, 29(1), 7-20.

Kovacs, M. (1992). Children's Depression Inventory (CDI) Manual. Toronto, ON: Multi-Health Systems.

Kovacs, M., \& Beck, A. T. (1977). An empirical-clinical approach toward a definition of childhood depression. In J. G. Schulterbrandt \& A. Raskin (Eds.), Depression in Childhood: Diagnosis, Treatment, and Conceptual Models (pp. 1-25). New York, NY: Raven Press.

Kowalski, R. M. (2000). Anxiety. In A. E. Kazdin (Ed.), Encyclopedia of Psychology. American Psychological Association and Oxford University Press.

Kremer, P., Elshaug, C., Leslie, E., Toumbourou, J. W., Patton, G. C., \& Williams, J. (2014). Physical activity, leisure-time screen use and depression among children and young adolescents. Journal of Science and Medicine in Sport, 17(2), 183187.

Kuźbicka, K., \& Rachoń, D. (2013). Bad eating habits as the main cause of obesity among children. Pediatric Endocrinology, Diabetes and Metabolism, 19(3), 106-110.

Kyu, H. H., Bachman, V. F., Alexander, L. T., Mumford, J. E., Afshin, A., Estep, K., ... Forouzanfar, M. H. (2016). Physical activity and risk of breast cancer, colon cancer, diabetes, ischemic heart disease, and ischemic stroke events: systematic review and dose-response meta-analysis for the Global Burden of Disease Study 2013. BMJ, 354, i3857.

Lambrick, D., Westrupp, N., Kaufmann, S., Stoner, L., \& Faulkner, J. (2016). The effectiveness of a high-intensity games intervention on improving indices of health in young children. Journal of Sports Sciences, 34(3), 190-198.

Lanzi, S., Codecasa, F., Cornacchia, M., Maestrini, S., Capodaglio, P., Brunani, A., ... Malatesta, D. (2015). Long maximal incremental tests accurately assess aerobic fitness in class II and III obese men. PLoS ONE, 10(4), 1-12.

Larun, L., Nordheim, L. V, Ekeland, E., Hagen, K. B., \& Heian, F. (2006). Exercise in prevention and treatment of anxiety and depression among children and young people (Review). The Cochrane Database of Systematic Reviews, (3).

Lau, P. W. C., Wong, D. P., Ngo, J. K., Liang, Y., Kim, C. G., \& Kim, H. S. (2014). Effects of high-intensity intermittent running exercise in overweight children. European Journal of Sport Science, 11, 1-9.

Lau, P. W. C., Yu, C. W., Lee, A., \& Sung, R. Y. T. (2004). The physiological and psychological effects of resistance training on Chinese obese adolescents. Journal of Exercise Science and Fitness, 2(2), 115-120.

Laursen, A. H., Kristiansen, O. P., Marott, J. L., Schnohr, P., \& Prescott, E. (2012). Intensity versus duration of physical activity: implications for the metabolic syndrome. A prospective cohort study. BMJ Open, 2, e001711.

Laursen, P. B., \& Jenkins, D. G. (2002). The scientific basis for high-intensity interval training: optimising training programmes and maximising performance in highly trained endurance athletes. Sports Medicine, 32(1), 53-73. 
Laursen, P. B., Shing, C. M., Peake, J. M., Coombes, J. S., \& Jenkins, D. G. (2002). Interval training program optimization in highly trained endurance cyclists. Medicine and Science in Sports and Exercise, 34(11), 1801-1807.

Lazaar, N., Aucouturier, J., Ratel, S., Rance, M., Meyer, M., \& Duché, P. (2007). Effect of physical activity intervention on body composition in young children: influence of body mass index status and gender. Acta Paediatrica, 96(9), 13151320 .

Le, M. T. H., Tran, T. D., Holton, S., Nguyen, H. T., Wolfe, R., \& Fisher, J. (2017). Reliability, convergent validity and factor structure of the DASS-21 in a sample of Vietnamese adolescents. PLoS ONE, 12(7), e0180557.

Lee, D., Artero, E. G., Sui, X., \& Blair, S. N. (2010). Mortality trends in the general population: the importance of cardiorespiratory fitness. Journal of Psychopharmacology, 24(4 Suppl), 27-35.

Lee, I.-M., \& Paffenbarger, R. S. (2000). Associations of light, moderate, and vigorous intensity physical activity with longevity: the Harvard Alumni Health Study. American Journal of Epidemiology, 151(3), 293-299.

Leith, L. M. (1994). Foundations of exercise and mental health. Morgantown, WV: Fitness Information Technology.

Leung, F. H., \& Savithiri, R. (2009). Spotlight on focus groups. Canadian Family Physician, 55(2), 218-219.

Liberati, A., Altman, D. G., Tetzlaff, J., Mulrow, C., Gøtzsche, P. C., Ioannidis, J. P. A., ... Moher, D. (2009). The PRISMA statement for reporting systematic reviews and meta-analyses of studies that evaluate health care interventions: explanation and elaboration. PLoS Medicine, 6(7), e1000100.

Lincoln, Y. S., \& Guba, E. G. (1985). Naturalistic inquiry. Beverly Hills, CA: Sage Publications.

Little, J. P., Gillen, J. B., Percival, M. E., Safdar, A., Tarnopolsky, M. A., Punthakee, Z., ... Gibala, M. J. (2011). Low-volume high-intensity interval training reduces hyperglycemia and increases muscle mitochondrial capacity in patients with type 2 diabetes. Journal of Applied Physiology, 111, 1554-1560.

Lobstein, T., Jackson-Leach, R., Moodie, M. L., Hall, K. D., Gortmaker, S. L., Swinburn, B. A., ... McPherson, K. (2015). Child and adolescent obesity: part of a bigger picture. The Lancet, 385(9986), 2510-2520.

Logan, G. R. M., Harris, N., Duncan, S., \& Schofield, G. (2014). A review of adolescent high-intensity interval training. Sports Medicine, 44(8), 1071-1085.

Lonsdale, C., Rosenkranz, R. R., Peralta, L. R., Bennie, A., Fahey, P., \& Lubans, D. R. (2013). A systematic review and meta-analysis of interventions designed to increase moderate-to-vigorous physical activity in school physical education lessons. Preventive Medicine, 56(2), 152-161.

Lovell, G. P., Ansari, W. E., \& Parker, J. K. (2010). Perceived exercise benefits and barriers of non-exercising female university students in the United Kingdom. International Journal of Environmental Research and Public Health, 7(3), 784798. 
Lovibond, S. H., \& Lovibond, P. F. (1995). Manual for the Depression Anxiety \& Stress Scales (2nd ed.). Sydney: Psychology Foundation.

Löwe, B., Decker, O., Müller, S., Brähler, E., Schellberg, D., Herzog, W., \& Herzberg, P. Y. (2008). Validation and standardization of the Generalized Anxiety Disorder Screener (GAD-7) in the general population. Medical Care, 46(3), 266-274.

Lowery, S. E., Kurpius, S. E. R., Befort, C., Blanks, E. H., Sollenberger, S., Nicpon, M. F., \& Huser, L. (2005). Body image, self-esteem, and health-related behaviors among male and female first year college students. Journal of College Student Development, 46(6), 612-623.

Lubans, D., Richards, J., Hillman, C., Faulkner, G., Beauchamp, M., Nilsson, M., ... Biddle, S. (2016). Physical activity for cognitive and mental health in youth: a systematic review of mechanisms. Pediatrics, 138(3), e20161642.

Luppino, F. S., de Wit, L. M., Bouvy, P. F., Stijnen, T., Cuijpers, P., Penninx, B. W. J. H., \& Zitman, F. G. (2010). Overweight, obesity, and depression: a systematic review and meta-analysis of longitudinal studies. Archives of General Psychiatry, 67(3), 220-229.

MacDonald, M. J., \& Currie, K. D. (2009). Interval exercise is a path to good health, but how much, how often and for whom? Clinical Science, 116, 315-316.

Machado, F. A., \& Denadai, B. S. (2011). Validity of maximum heart rate prediction equations for children and adolescents. Arquivos Brasileiros de Cardiologia, 97(2), 136-140.

Mackintosh, K. A., Knowles, Z. R., Ridgers, N. D., \& Fairclough, S. J. (2011). Using formative research to develop CHANGE!: a curriculum-based physical activity promoting intervention. BMC Public Health, 11(1), 831.

Mackintosh, K. A., Niezen, G., \& Eslambolchilar, P. (2016). Mission Possible: using ubiquitous social goal sharing technology to promote physical activity in children. Movement, Health \& Exercise, 5(2), 1-14.

Mahon, A. D., Marjerrison, A. D., Lee, J. D., Woodruff, M. E., \& Hanna, L. E. (2010). Evaluating the prediction of maximal heart rate in children and adolescents. Research Quarterly for Exercise and Sport, 81(4), 466-471.

Mandigo, J. L., Holt, N., Anderson, A., \& Sheppard, J. (2008). Children's motivational experiences following autonomy-supportive games lessons. European Physical Education Review, 14(3), 407-425.

Manson, J. E., Greenland, P., LaCroix, A. Z., Stefanick, M. L., Mouton, C. P., Oberman, A., ... Siscovick, D. S. (2002). Walking compared with vigorous exercise for the prevention of cardiovascular events in women. The New England Journal of Medicine, 347(10), 716-725.

Markowitz, S., Friedman, M. A., \& Arent, S. M. (2008). Understanding the relation between obesity and depression : causal mechanisms and implications for treatment. Clinical Psychology: Science and Practice, 15(1), 1-20.

Marsh, H., Debus, R., \& Bornholt, L. (2008). Validating young children's selfconcept responses: methodological ways and means to understand their 
responses. In D. M. Teti (Ed.), Handbook of Research Methods in Developmental Science (pp. 138-160). Oxford, UK: Blackwell Publishing.

Martinez-Gomez, D., Ruiz, J. R., Ortega, F. B., Casajús, J. A., Veiga, O. L., Gonza, M., ... Sjöström, M. (2010). Recommended levels and intensities of physical activity to avoid low-cardiorespiratory fitness in European adolescents: the HELENA study. American Journal of Human Biology, 22, 750-756.

Martínez-Vizcaíno, V., Sánchez-López, M., Notario-Pacheco, B., Salcedo-Aguilar, F., Solera-Martínez, M., Franquelo-Morales, P., ... Rodríguez-Artalejo, F. (2014). Gender differences on effectiveness of a school-based physical activity intervention for reducing cardiometabolic risk: a cluster randomized trial. International Journal of Behavioral Nutrition and Physical Activity, 11(154).

Mattocks, C., Ness, A. R., Leary, S. D., Tilling, K., Blair, S. N., Shield, J., ... Riddoch, C. J. (2008). Use of accelerometers in a large field-based study of children: protocols, design issues, and effects on precision. Journal of Physical Activity \& Health, 5(1), S98-S111.

Matza, L. S., Swensen, A. R., Flood, E. M., Secnik, K., \& Leidy, N. K. (2004). Assessment of health-related quality of life in children: a review of conceptual, methodological, and regulatory issues. Value in Health, 7(1), 79-92.

Mayorga-Vega, D., Aguilar-Soto, P., \& Viciana, J. (2015). Criterion-related validity of the 20-M shuttle run test for estimating cardiorespiratory fitness: a metaanalysis. Journal of Sports Science and Medicine, 14, 536-547.

McArdle, W. D., Katch, F. I., \& Katch, V. L. (1996). Exercise physiology. Baltimore, MD: Williams \& Wilkins.

McCrone, P., Dhanasiri, S., Patel, A., Knapp, M., \& Lawton-Smith, S. (2008). Paying the price: the cost of mental health care in England to 2026. King's Fund.

McDowell, C. P., MacDonncha, C., \& Herring, M. P. (2017). Brief report: associations of physical activity with anxiety and depression symptoms and status among adolescents. Journal of Adolescence, 55, 1-4.

McGuire, M. T., Neumark-Sztainer, D. R., \& Story, M. (2002). Correlates of time spent in physical activity and television viewing in a multi-racial sample of adolescents. Pediatric Exercise Science, 14, 75-86.

Mclaughlin, L., \& Hinyard, L. J. (2014). The relationship between health-related quality of life and body mass index. Western Journal of Nursing Research, 36(8), 989-1001.

McMahon, E. M., Corcoran, P., O’Regan, G., Keeley, H., Cannon, M., Carli, V., ... Wasserman, D. (2017). Physical activity in European adolescents and associations with anxiety, depression and well-being. European Child and Adolescent Psychiatry, 26(1), 111-122.

McMurray, R. G., Harrell, J. S., Bangdiwala, S. I., Bradley, C. B., Deng, S., \& Levine, A. (2002). A school-based intervention can reduce body fat and blood pressure in young adolescents. The Journal of Adolescent Health, 31(02), 125132. 
McNarry, M. A., Mackintosh, K. A., \& Stoedefalke, K. (2014). Longitudinal investigation of training status and cardiopulmonary responses in pre- and earlypubertal children. European Journal of Applied Physiology, 114(8), 1573-1580.

McNarry, M. A., Welsman, J. R., \& Jones, A. M. (2011). The influence of training and maturity status on girls' responses to short-term, high-intensity upper- and lower-body exercise. Applied Physiology, Nutrition, and Metabolism, 36(3), 344-352.

Meyer, A. A., Kundt, G., Lenschow, U., Schuff-Werner, P., \& Kienast, W. (2006). Improvement of early vascular changes and cardiovascular risk factors in obese children after a six-Month exercise program. Journal of the American College of Cardiology, 48(9), 1865-1870.

Miadich, S. A., Everhart, R. S., Borschuk, A. P., Winter, M. A., \& Fiese, B. H. (2015). Quality of life in children with asthma: a developmental perspective. Journal of Pediatric Psychology, 40(7), 672-679.

Michalos, A. C. (2004). Social indicators research and health-related quality of life research. Social Indicators Research, 65, 27-72.

Mirwald, R. L. (1981). Longitudinal comparison of aerobic power in active and inactive boys aged 7.0 to 17.0 years. Annals of Human Biology, 8(5), 405-414.

Mirwald, R. L., Baxter-Jones, A. D. G., Bailey, D. A., \& Beunen, G. P. (2002). An assessment of maturity from anthropometric measurements. Medicine and Science in Sports and Exercise, 34(4), 689-694.

Moher, D., Dulberg, C. S., \& Wells, G. A. (1994). Statistical power, sample size, and their reporting in randomized controlled trials. Journal of the American Medical Association, 272(2), 122-124.

Monyeki, M. A., Neetens, R., Moss, S. J., \& Twisk, J. (2012). The relationship between body composition and physical fitness in 14 year old adolescents residing within the Tlokwe local municipality, South Africa: the PAHL study. BMC Public Health, 12, 374.

Morales, P. F., Sánchez-López, M., Moya-Martínez, P., García-Prieto, J. C., Martínez-Andrés, M., García, N. L., \& Martínez-Vizcaíno, V. (2013). Healthrelated quality of life, obesity, and fitness in schoolchildren: the Cuenca study. Quality of Life Research, 22(7), 1515-1523.

Morgan, M., Gibbs, S., Maxwell, K., \& Britten, N. (2002). Hearing children's voices: methodological issues in conducting focus groups with children aged 711 years. Qualitative Research, 2(1), 5-20.

Mossman, S. A., Luft, M. J., Schroeder, H. K., Varney, S. T., Fleck, D. E., Barzman, D. H., ... Strawn, J. R. (2017). The Generalized Anxiety Disorder 7-item scale in adolescents with generalized anxiety disorder: signal detection and validation. Annals of Clinical Psychiatry, 29(4), 227-234.

Myers, J., McAuley, P., Lavie, C. J., Despres, J.-P., Arena, R., \& Kokkinos, P. (2015). Physical activity and cardiorespiratory fitness as major markers of cardiovascular risk: their independent and interwoven importance to health status. Progress in Cardiovascular Diseases, 57(4), 306-314. 
Myers, K., \& Winters, N. C. (2002). Ten-year review of rating scales. II: scales for internalizing disorders. Journal of the American Academy of Child and Adolescent Psychiatry, 41(6), 634-659.

Nakagawa, S., \& Cuthill, I. C. (2007). Effect size, confidence interval and statistical significance: A practical guide for biologists. Biological Reviews of the Cambridge Philosophical Society, 82(4), 591-605.

National Assembly for Wales Children Young People and Education Committee. (2014). Inquiry into child and adolescent mental health services (CAMHS). Cardiff.

National Collaborating Centre for Mental Health. (2011). Common mental health problems: identification and pathways to care. Manchester, UK.

National Collaborating Centre for Mental Health and The National Institute for Health and Care Excellence. (2010). Depression: the treatment and management of depression in adults (updated edition). Leicester: The British Psychological Society and The Royal College of Psychiatrists.

Nayir, T., Uskun, E., Yürekli, M. V, Çelick, A., \& Okyay, R. A. (2016). Does body image affect quality of life?: a population based study. PLOS ONE, 11(9), e0163290.

Nelwamondo, F. V, Mohamed, S., \& Marwala, T. (2007). Missing data: a comparison of neural network and expectation maximization techniques. Current Science, 93(11), 1514-1521.

Netz, Y., Wu, M. J., Becker, B. J., \& Tenenbaum, G. (2005). Physical activity and psychological well-being in advanced age: a meta-analysis of intervention studies. Psychology and Aging, 20(2), 272-284.

Nevill, A. M., Stewart, A. D., Olds, T., \& Holder, R. (2006). Relationship between adiposity and body size reveals limitations of BMI. American Journal of Physical Anthropology, 129(1), 151-156.

Ng, M., Fleming, T., Robinson, M., Thomson, B., Graetz, N., Margono, C., ... Gakidou, E. (2014). Global, regional, and national prevalence of overweight and obesity in children and adults during 1980-2013: a systematic analysis for the global burden of disease study 2013. The Lancet, 384(9945), 766-781.

Nolen-Hoeksema, S. (2001). Gender differences in depression. Current Directions in Psychological Science, 10(5), 173-176.

Norris, R., Carroll, D., \& Cochrane, R. (1992). The effects of physical activity and exercise training on psychological stress and well-being in an adolescent population. Journal of Psychosomatic Research, 36(1), 55-65.

Nourry, C., Deruelle, F., Guinhouya, C., Baquet, G., Fabre, C., Bart, F., ... Mucci, P. (2005). High-intensity intermittent running training improves pulmonary function and alters exercise breathing pattern in children. European Journal of Applied Physiology, 94(4), 415-423.

Obert, P., Mandigouts, S., Nottin, S., Vinet, A., N'Guyen, L. D., \& Lecoq, A. M. (2003). Cardiovascular responses to endurance training in children: effect of gender. European Journal of Clinical Investigation, 33(3), 199-208. 
Office for National Statistics. (2017). Deaths Registered in England and Wales (Series DR): 2016. London, UK.

Olivares, P. R., Cossio-Bolaños, M. A., Gomez-Campos, R., Almonacid-Fierro, A., \& Garcia-Rubio, J. (2015). Influence of parents and physical education teachers in adolescent physical activity. International Journal of Clinical and Health Psychology, 15(2), 113-120.

Olson, L. M., Lara, M., \& Frintner, M. P. (2004). Measuring health status and quality of life for US children: relationship to race, ethnicity, and income status. Ambulatory Pediatrics, 4(4), 377-386.

Ortega, F. B., Artero, E. G., Ruiz, J. R., Vicente-Rodriguez, G., Bergman, P., Hagströmer, M., ... Castillo, M. J. (2008). Reliability of health-related physical fitness tests in European adolescents. The HELENA Study. International Journal of Obesity, 32, S49-S57.

Osman, A., Hoffman, J., Barrios, F. X., Kopper, B. A., Breitenstein, J. L., \& Hahn, S. K. (2002). Factor structure, reliability, and validity of the Beck Anxiety Inventory in adolescent psychiatric inpatients. Journal of Clinical Psychology, $58(4), 443-456$.

Ostojic, S. M., Stojanovic, M., Jukic, I., Pasalic, E., \& Jourkesh, M. (2009). The effects of six weeks of training on physical fitness and performance in teenage and mature top-level soccer players. Biology of Sport, 26(4), 379-387.

Palmer, H. S. (2010). Exercise training for a time-poor generation: enhanced skeletal muscle mitochondrial biogenesis. Journal of Physiology, 588(11), 1817-1818.

Parfitt, G., \& Hughes, S. (2009). The exercise intensity-affect realationship: evidence and implications for exercise behaviour. Journal of Exercise Science \& Fitness, 7(2), S34-S41.

Parfitt, G., Pavey, T., \& Rowlands, A. V. (2009). Children's physical activity and psychological health: the relevance of intensity. Acta Paediatrica, 98(6), 10371043.

Parikh, T., \& Stratton, G. (2011). Influence of intensity of physical activity on adiposity and cardiorespiratory fitness in 518 year olds. Sports Medicine, 41(6), 477-488.

Parker, A. G., Hetrick, S. E., Jorm, A. F., Mackinnon, A. J., McGorry, P. D., Yung, A. R., ... Purcell, R. (2016). The effectiveness of simple psychological and physical activity interventions for high prevalence mental health problems in young people: a factorial randomised controlled trial. Journal of Affective Disorders, 196, 200-209.

Pasquali, R., \& Vicennati, V. (2000). Activity of the hypothalamic-pituitary-adrenal axis in different obesity phenotypes. International Journal of Obesity, 24(Suppl 2), S47-S49.

Passer, M. W. (1981). Children in sport: participation motives and psychological stress. Quest, 33(2), 231-244.

Pate, R. R., Pratt, M., Blair, S. N., Haskell, W. L., Macera, C. A., Bouchard, C., ... Wilmore, J. H. (1995). Physical activity and public health: a recommendation 
from the Centers for Disease Control and Prevention and the American College of Sports Medicine. Journal of the American Medical Association, 273(5), 402407.

Pate, R. R., Wang, C., Dowda, M., Farrell, S. W., \& O’Neill, J. R. (2006).

Cardiorespiratory fitness levels among US youth 12 to 19 years of age. Archives of Pediatrics \& Adolescent Medicine, 160(10), 1005-1012.

Patrick, J., Dyck, M., \& Bramston, P. (2010). Depression Anxiety Stress Scale: is it valid for children and adolescents? Journal of Clinical Psychology, 66(9), 9961007.

Paulhus, D. L., \& Vazire, S. (2005). The self-report method. In R. W. Robins, R. C. Fraley, \& R. F. Krueger (Eds.), Handbook of research methods in personality psychology (pp. 224-239). New York: Guilford.

Peluso, M. A. M., \& de Andrade, L. H. S. G. (2005). Physical activity and mental health: the association between exercise and mood. Clinics, 60(1), 61-70.

Penedo, F. J., \& Dahn, J. R. (2005). Exercise and well-being: a review of mental and physical health benefits associated with physical activity. Current Opinion in Psychiatry, 18(2), 189-193.

Petruzzello, S. J., Landers, D. M., Hatfield, B. D., Kubitz, K. A., \& Salazar, W. (1991). A meta-analysis on the anxiety-reducing effects of acute and chronic exercise. Sports Medicine, 11(3), 143-182.

Phillips, W. T., Kiernan, M., \& King, A. C. (2003). Physical activity as a nonpharmacological treatment for depression: a review. Complementary Health Practice Review, 8(2), 139-152.

Piccinelli, M., \& Wilkinson, G. (1999). Gender differences in depression: critical review. British Journal of Psychiatry, 177, 486-493.

Piers, E. V, \& Harris, D. B. (1969). The Piers-Harris Children's Self-Concept Scale. Los Angeles, CA: Western Psychological Services.

Porcellato, L., Dughill, L., \& Springett, J. (2002). Using focus groups to explore children's perceptions of smoking: reflections on practice. Health Education, 102(6), 310-320.

Post, M. W. M. (2014). Definitions of quality of life: what has happened and how to move on. Topic in Spinal Cord Injury Rehabilitation, 20(3), 167-180.

Poznanski, E., \& Mokros, H. (1996). Children's Depression Rating Scale-Revised $(C D R S-R)$. Los Angeles, CA: WPS.

Prochaska, J. J., Sallis, J. F., \& Long, B. (2001). A physical activity screening measure for use with adolescents in primary care. Archives of Pediatrics and Adolescent Medicine, 155(5), 554-559.

Proper, K. I., van der Beek, A. J., Hildebrandt, V. H., Twisk, J. W., \& Van, M. W. (2003). Short term effect of feedback on fitness and health measurements on self reported appraisal of the stage of change. British Journal of Sports Medicine, 37, 529-534.

Racil, G., Coquart, J. B., Elmontassar, W., Haddad, M., Chaouachi, A., Amri, M., \& 
Chamari, K. (2016). Greater effects of high- compared with moderate-intensity interval training on cardio-metabolic variables, blood leptin concentration and ratings of perceived exertion in obese adolescent females. Biology of Sport, $33(2), 145-152$.

Radloff, L. S. (1977). The CES-D Scale: a self-report depression scale for research in the general population. Applied Psychological Measurement, 1(3), 385-401.

Radloff, L. S. (1991). The use of the Center for Epidemiologic Studies Depression Scale in adolescents and young adults. Journal of Youth and Adolescence, 20(2), 149-166.

Raedeke, T. D. (2007). The relationship between enjoyment and affective responses to exercise. Journal of Applied Psychology, 19, 105-115.

Ransford, C. P. (1982). A role for amines in the antidepressant effect of exercise: a review. Medicine and Science in Sports and Exercise, 4(1), 1-10.

Rasmussen, A. R., Wohlfahrt-Veje, C., Tefre de Renzy-Martin, K., Hagen, C. P., Tinggaard, J., Mouritsen, A., ... Main, K. M. (2015). Validity of selfassessment of pubertal maturation. Pediatrics, 135(1), 86-93.

Ratel, S., Lazaar, N., Dore, E., Baquet, G., Williams, C. A., Berthoin, S., ... Duche, P. (2004). High-intensity intermittent activities at school: controversies and facts. Journal of Sports Medicine and Physical Fitness, 44(3), 272-280.

Ravens-Sieberer, U., Erhart, M., Wille, N., Wetzel, R., Nickel, J., \& Bullinger, M. (2006). Generic health-related quality-of-life assessment in children and adolescents: methodological considerations. Pharmacoeconomics, 24(12), 1199-1220.

Ravens-Sieberer, U., Gosch, A., Rajmil, L., Erhart, M., Bruil, J., Duer, W., ... Tountas, Y. (2005). KIDSCREEN-52 quality-of-life measure for children and adolescents. Expert Review of Pharmacoeconomics \& Outcomes Research, 5(3), $353-364$.

Ravens-Sieberer, U., Herdman, M., Devine, J., Otto, C., Bullinger, M., Rose, M., \& Klasen, F. (2014). The European KIDSCREEN approach to measure quality of life and well-being in children: development, current application, and future advances. Quality of Life Research, 23(3), 791-803.

Ravens-Sieberer, U., Karow, A., Barthel, D., \& Klasen, F. (2014). How to assess quality of life in child and adolescent psychiatry. Dialogues in Clinical Neuroscience, 16(2), 147-158.

Rebar, A. L., Stanton, R., Geard, D., Short, C., Duncan, M. J., \& Vandelanotte, C. (2015). A meta-meta-analysis of the effect of physical activity on depression and anxiety in non-clinical adult populations. Health Psychology Review, 9(3), $366-378$.

Rees, D. I., \& Sabia, J. J. (2010). Exercise and adolescent mental health: new evidence from longitudinal data. Journal of Mental Health Policy and Economics, 13(1), 13-25.

Reichert, F. F., Barros, A. J. D., Domingues, M. R., \& Hallal, P. C. (2007). The role of perceived personal barriers to engagement in leisure-time physical activity. 
American Journal of Public Health, 97(3), 515-519.

Reynolds, C. R., \& Richmond, B. O. (1985). Revised Children's Manifest Anxiety

Scale. Los Angeles, CA: Western Psychological Services.

Reynolds, S., Wilson, C., Austin, J., \& Hooper, L. (2012). Effects of psychotherapy for anxiety in children and adolescents: a meta-analytic review. Clinical Psychology Review, 32(4), 251-262.

Rich, C., Geraci, M., Griffiths, L., Sera, F., Dezateux, C., \& Cortina-Borja, M. (2013). Quality control methods in accelerometer data processing: defining minimum wear time. PLoS ONE, 8(6), e67206.

Ridgers, N. D., Knowles, Z. R., \& Sayers, J. (2012). Encouraging play in the natural environment: a child-focused case study of Forest School. Children's Geographies, 10(1), 49-65.

Ridgers, N. D., Timperio, A., Cerin, E., \& Salmon, J. (2014). Compensation of physical activity and sedentary time in primary school children. Medicine and Science in Sports and Exercise, 46(8), 1564-1569.

Ries, A. V, Gittelsohn, J., Voorhees, C. C., Roche, K. M., Clifton, K. J., \& Astone, N. M. (2008). The environment and urban adolescents' use of recreational facilities for physical activity: a qualitative study. American Journal of Health Promotion, 23, 43-51.

Riley, A. W. (2004). Evidence that school-age children can self-report on their health. Ambulatory Pediatrics, 4(Suppl 4), 371-376.

Rippe, J. M., Price, J. M., Hess, S. A., Kline, G., Demers, K. A., Damitz, S., ... Freedsoni, P. (1998). Improved psychological well-being, quality of life, and health practices in moderately overweight women participating in a 12-week structured weight loss program. Obesity Research, 6(3), 208-218.

Roberts, R. E., Deleger, S., Strawbridge, W. J., \& Kaplan, G. A. (2003). Prospective association between obesity and depression: evidence from the Alameda County Study. International Journal of Obesity, 27, 514-521.

Robertson, R., Wenzel, L., Thompson, J., \& Charles, A. (2017). Understanding NHS financial pressures. London, UK.

Rokholm, B., Baker, J. L., \& Sørensen, T. I. A. (2010). The levelling off of the obesity epidemic since the year 1999 - a review of evidence and perspectives. Obesity Reviews, 11(12), 835-846.

Rosenkranz, S. K., Rosenkranz, R. R., Hastmann, T. J., \& Harms, C. A. (2012). High-intensity training improves airway responsiveness in inactive nonasthmatic children: evidence from a randomized controlled trial. Journal of Applied Physiology, 112(7), 1174-1183.

Rosner, B. (2011). Fundamentals of Biostatistics (7th ed.). Boston, MA: Brooks/Cole.

Ross, C. E. (1994). Overweight and depression. Journal of Health and Social Behavior, 35(1), 63-79.

Ross, L. M., Porter, R. R., \& Durstine, J. L. (2016). High-intensity interval training 
(HIIT) for patients with chronic diseases. Journal of Sport and Health Science, $5,1-6$.

Rothberg, A. E., Mcewen, L. N., Kraftson, A. T., Neshewat, G. M., Fowler, E., Burant, C. F., \& Herman, W. H. (2015). The impact of weight loss on healthrelated quality-of-life: implications for cost-effectiveness analyses. Quality of Life Research, 23(4), 1371-1376.

Rothon, C., Edwards, P., Bhui, K., Viner, R. M., Taylor, S., \& Stansfeld, S. A. (2010). Physical activity and depressive symptoms in adolescents: a prospective study. BMC Medicine, 8(32).

Rowland, T. W. (1998). The biological basis of physical activity. Medicine and Science in Sports and Exercise, 30(3), 392-399.

Ruggero, C. J., Petrie, T., Sheinbein, S., Greenleaf, C., \& Martin, S. (2015). Cardiorespiratory fitness may help in protecting against depression among middle school adolescents. Journal of Adolescent Health, 57(1), 60-65.

Ruiz, J. R., Artero, E. G., Ortega, F. B., Sjo, M., Suni, J., \& Castillo, M. J. (2009). Predictive validity of health-related fitness in youth: a systematic review. British Journal of Sports Medicine, 43, 909-923.

Rumsby, B. (2015). Alarming fall in school PE lessons casts doubt over government's commitment to tackling obesity crisis. The Telegraph. Retrieved from http://www.telegraph.co.uk/sport/othersports/schoolsports/11343801/Alarmingfall-in-school-PE-lessons-casts-doubt-over-governments-commitment-totackling-obesity-crisis.html

Ryan, R. M., \& Deci, E. L. (2000). Self-determination theory and the facilitation of intrinsic motivation, social development, and well-being, 55(1), 68-78.

Ryan, R. M., Frederick, C. M., Lepes, D., Rubio, N., \& Sheldon, K. M. (1997). Intrinsic motivation and exercise adherence. International Journal of Sport Psychology, 28, 335-354.

Ryley, A. (2013). Children's BMI, overweight and obesity. In C. R. Mindell (Ed.), Health Survey for England - 2012. London: NHS England.

Saanijoki, T., Tuominen, L., Tuulari, J. J., Nummenmaa, L., Arponen, E., Kalliokoski, K., \& Hirvonen, J. (2017). Opioid release after high-intensity interval training in healthy human subjects. Neuropsychopharmacology.

Sach, T. H., Barton, G. R., Doherty, M., Muir, K. R., Jenkinson, C., \& Avery, A. J. (2007). The relationship between body mass index and health- related quality of life: comparing the EQ-5D, EuroQol VAS and SF-6D. International Journal of Obesity, 31, 189-196.

Sahoo, K., Sahoo, B., Choudhury, A. K., Sofi, N. Y., Kumar, R., \& Bhadoria, A. S. (2015). Childhood obesity: Causes and consequences. Journal of Family Medicine and Primary Care, 4(2), 187-192.

Saint-Maurice, P. F., Troiano, R. P., Berrigan, D., Kraus, W. E., \& Matthews, C. E. (2018). Volume of light versus moderate-to-vigorous physical activity: similar benefits for all-cause mortality? Journal of the American Heart Association, 
7(7), e008815.

Sallis, J. F., Prochaska, J. J., \& Taylor, W. C. (2000). A review of correlates of physical activity of children and adolescents. Medicine and Science in Sports and Exercise, 32(5), 963-975.

Sandercock, G., Ogunleye, A., \& Voss, C. (2015). Six-year changes in body mass index and cardiorespiratory fitness of English schoolchildren from an affluent area. International Journal of Obesity, 39(10), 1504-1507.

Sandercock, G., Voss, C., McConnell, D., \& Rayner, P. (2010). Ten year secular declines in the cardiorespiratory fitness of affluent English children are largely independent of changes in body mass index. Archives of Disease in Childhood, 95(1), 46-47.

Saunders, R. P., Pate, R. R., Dowda, M., Ward, D. S., Epping, J. N., \& Dishman, R. K. (2012). Assessing sustainability of Lifestyle Education for Activity Program (LEAP). Health Education Research, 27(2), 319-330.

Scarborough, P., Bhatnagar, P., Wickramasinghe, K. K., Allender, S., Foster, C., \& Rayner, M. (2011). The economic burden of ill health due to diet, physical inactivity, smoking, alcohol and obesity in the UK: an update to 2006-07 NHS costs. Journal of Public Health, 33(4), 527-535.

Schalock, R. L. (1996). Reconsidering the conceptualization and measurement of quality of life. In R. L. Schalock (Ed.), Quality of Life, Volume I:

Conceptualization and Measurement (pp. 123-139). Washington DC: American Association on Mental Retardation.

Schmidt, L. J., Garratt, A. M., \& Fitzpatrick, R. (2000). Instruments for Mental Health: A Review. London, UK: Patient-Reported Health Instruments Group.

Schmitz, K. E., Hovell, M. F., Nichols, J. F., Irvin, V. L., Keating, K., Simon, G. M., ... Jones, K. L. (2004). A validation study of early adolescents' pubertal selfassessments. Journal of Early Adolescence, 24(4), 357-384.

Scholes, S. (2016). Health survey for England 2015: physical activity in children. London, UK.

Schönfeld, P., Brailovskaia, J., Bieda, A., Zhang, X. C., \& Margraf, J. (2016). The effects of daily stress on positive and negative mental health: mediation through self-efficacy. International Journal of Clinical and Health Psychology, 16(1), $1-10$.

Scott, K. M., Bruffaerts, R., Simon, G. E., Alonso, J., Angermeyer, M., Girolamo, G. De, ... Von Korff, M. (2008). Obesity and mental disorders in the general population: results from the world mental health surveys. International Journal of Obesity, 32, 192-200.

Sharpe, H., Patalay, P., Fink, E., Vostanis, P., Deighton, J., \& Wolpert, M. (2016). Exploring the relationship between quality of life and mental health problems in children: implications for measurement and practice. European Child \& Adolescent Psychiatry, 25(6), 659-667.

Shephard, R. J. (2003). Limits to the measurement of habitual physical activity by questionnaires. British Journal of Sports Medicine, 37, 197-206. 
Shepherd, S. O., Wilson, O. J., Taylor, A. S., Thøgersen-Ntoumani, C., Adlan, A. M., Wagenmakers, A. J. M., \& Shaw, C. S. (2015). Low-volume high-intensity interval training in a gym setting improves cardio-metabolic and psychological health. PLOS ONE, 10(9), e0139056.

Shiroma, E. J., Cook, N. R., Manson, J. E., Moorthy, M. V, Buring, J. E., Rimm, E. B., \& Lee, I.-M. (2017). Strength training and the risk of type 2 diabetes and cardiovascular disease. Medicine and Science in Sports and Exercise, 49(1), 4046.

Sigal, R. J., Alberga, A. S., Goldfield, G. S., Prud'homme, D., Hadjiyannakis, S., Gougeon, R., ... Kenny, G. P. (2014). Effects of aerobic training, resistance training, or both on percentage body fat and cardiometabolic risk markers in obese adolescents: the healthy eating aerobic and resistance training in youth randomized clinical trial. Journal of the American Medical Association Pediatrics, 168(11), 1006-1014.

Simmons, M., Wilkinson, P., \& Dubicka, B. (2015). Measurement issues: depression measures in children and adolescents. Child and Adolescent Mental Health, 20(4), 230-241.

Singh, N. A., Stavrinos, T. A., Scarbek, Y., Galambos, G., Liber, C., \& Singh, M. A. F. (2005). A randomized controlled trial of high versus low intensity weight training versus general practitioner care for clinical depression in older adults. Journals of Gerontology Series A-Biological Sciences and Medical Sciences, 60(6), 768-776.

Skinner, A. C., Perrin, E. M., Moss, L. A., \& Skelton, J. A. (2015). Cardiometabolic risks and severity of obesity in children and young adults. The New England Journal of Medicine, 373, 1307-1317.

Slater, A., \& Tiggemann, M. (2010). "Uncool to do sport": A focus group study of adolescent girls' reasons for withdrawing from physical activity. Psychology of Sport \& Exercise, 11, 619-626.

Sloan, R. A., Sawada, S. S., Martin, C. K., Church, T., \& Blair, S. N. (2009). Associations between cardiorespiratory fitness and health-related quality of life. Health and Quality of Life Outcomes, 7(47).

Sluijs, E. M., Kok, G. J., \& van der Zee, J. (1993). Correlates of exercise comoliance in physical therapy. Physical Therapy, 73(11), 771-786.

Smith, B., \& McGannon, K. R. (2017). Developing rigor in qualitative research: problems and opportunities within sport and exercise psychology. International Review of Sport and Exercise Psychology.

Smith, J. K., \& Hodkinson, P. (2009). Challenging neorealisim: a response to Hammersley. Qualitative Inquiry, 15(1), 30-39.

Smithson, J. (2000). Using and analysing focus groups: limitations and possibilities. International Journal of Social Research Methodology, 3(2), 103-119.

Solans, M., Pane, S., Estrada, M.-D., Serra-Sutton, V., Berra, S., Herdman, M., ... Rajmil, L. (2008). Health-related quality of life measurement in children and adolescents: a systematic review of generic and disease-specific instruments. 
Value in Health, 11(4), 742-764.

Sperlich, B., De Marées, M., Koehler, K., Linville, J., Holmberg, H., \& Mester, J.

(2011). Effects of 5 weeks of high-intensity interval training vs. volume training in 14-year-old soccer players. Journal of Strength and Conditioning Research, 25(5), 1271-1278.

Spielberger, C. D. (1973). Manual for the State-Trait Anxiety Inventory for Children. Palo Alto, CA: Consulting Psychologisits Press.

Spielberger, C. D., \& Edwards, C. D. (1973). STAIC Preliminary Manual for the State-Trait Anxiety Inventory for Children ("How I Feel Questionnaire"). Sunnyvale, CA: Consulting Psychologists Press.

Spielberger, C. D., Gorsuch, R. L., \& Lushene, R. E. (1970). Manual for the StateTrait Anxiety Inventory (self-evaluation questionnaire). Palo Alto, CA: Consulting Psychologists Press.

Spitzer, R. L., Kroenke, K., Williams, J. B. W., \& Löwe, B. (2006). A brief measure for assessing generalized anxiety disorder: the GAD-7. Archives of Internal Medicine, 166(10), 1092-1097.

Stavrakakis, N., Roest, A. M., Verhulst, F., Ormel, J., de Jonge, P., \& Oldehinkel, A. J. (2013). Physical activity and onset of depression in adolescents: a prospective study in the general population cohort TRAILS. Journal of Psychiatric Research, 47(10), 1304-1308.

Stephens, T. (1988). Physical activity and mental health in the United States and Canada: evidence from four population surveys. Preventive Medicine, 17(1), $35-47$.

Stevens, J., Murray, D. M., Catellier, D. J., Hannan, P. J., Lytele, L. A., Elder, J. P., ... Webber, L. S. (2005). Design of the Trial of Activity in Adolescent Girls (TAAG). Contemporary Clinical Trials, 26(2), 223-233.

Stewart, A., Marfell-Jones, M., Olds, T., \& de Ridder, H. (2011). International standards for anthropometric assessment. Lower Hutt, NZ: International Society for the Advancement of Kinanthropometry.

Stockings, E., Degenhardt, L., Yi, Y., Mihalopoulos, C., Liu, A., Hobbs, M., \& Patton, G. (2015). Symptom screening scales for detecting major depressive disorder in children and adolescents: a systematic review and meta-analysis of reliability, validity and diagnostic utility. Journal of Affective Disorders, 174, 447-463.

Stonerock, G. L., Hoffman, B. M., Smith, P. J., \& Blumenthal, J. A. (2015). Exercise as treatment for anxiety: systematic review and analysis. Annals of Behavioral Medicine, 49(4), 542-556.

Stratton, G., Canoy, D., Boddy, L. M., Taylor, S. R., Hackett, A. F., \& Buchan, I. E. (2007). Cardiorespiratory fitness and body mass index of 9 - 11-year-old English children: a serial cross-sectional study from 1998 to 2004. International Journal of Obesity, 31, 1172-1178.

Strauss, R. S., Rodzilsky, D., Burack, G., \& Colin, M. (2001). Psychosocial correlates of physical activity in healthy children. Archives of Pediatrics \& 
Adolescent Medicine, 155(8), 897-902.

Ströhle, A., Höfler, M., Pfister, H., Müller, A.-G., Hoyer, J., Wittchen, H.-U., \& Lieb, R. (2007). Physical activity and prevalence and incidence of mental disorders in adolescents and young adults. Psychological Medicine, 37(11), 1657-1666.

Swallen, K. C., Reither, E. N., Haas, S. A., \& Meier, A. M. (2005). Overweight, obesity, and health-related quality of life among adolescents: the National Longitudinal Study of Adolescent Health. Pediatrics, 115(2), 340-347.

Tajik, E., Abd Latiff, L., Adznam, S. N., Awang, H., Yit Siew, C., \& Abu Bakar, A. S. (2017). A study on level of physical activity, depression, anxiety and stress symptoms among adolescents. The Journal of Sports Medicine and Physical Fitness, 57(10), 1382-1387.

Tan, Z. S., Spartano, N. L., Beiser, A. S., DeCarli, C., Auerbach, S. H., Vasan, R. S., \& Seshadri, S. (2017). Physical activity, brain volume, and dementia risk: the Framingham Study. Journals of Gerontology: Medical Sciences, 72(6), 789795.

Tanaka, H., Monahan, K. D., \& Seals, D. R. (2001). Age-predicted maximal heart rate revisited. Journal of the American College of Cardiology, 37(1), 153-156.

Tanasescu, M., Leitzmann, M. F., Rimm, E. B., Willett, W. C., Stampfer, M. J., \& $\mathrm{Hu}, \mathrm{F}$. B. (2002). Exercise type and intensity in relation to coronary heart disease in men. Journal of the American Medical Association, 288(16), 19942000 .

Tanner, J. M. (1962). Growths of adolescence. Oxford, UK: Blackwell Scientific.

Tao, F. B., Xu, M. L., Kim, S. D., Sun, Y., Su, P. Y., \& Huang, K. (2007). Physical activity might not be the protective factor for health risk behaviours and psychopathological symptoms in adolescents. Journal of Paediatrics and Child Health, 43(11), 762-767.

Taylor, K. L. (2014). Project FFAB (Fun Fast Activity Blasts): effect of a novel school-based high-intensity interval training intervention on cardiometabolic risk markers and physical activity levels in adolescents. Teeside University.

Taylor, K. L., Weston, M., \& Batterham, A. M. (2015). Evaluating intervention fidelity: an example from a high-intensity interval training study. PLoS ONE, 10(4), e0125166.

Telenius, E. W., Engedal, K., \& Bergland, A. (2015). Long-term effects of a 12 weeks high-intensity functional exercise program on physical function and mental health in nursing home residents with dementia: a single blinded randomized controlled trial. BMC Geriatrics, 15(1), 158.

Testa, M. A., \& Simonson, D. C. (1996). Assessment of quality-of-life outcomes. The New England Journal of Medicine, 334(13), 835-840.

Thabrew, H., Mcdowell, H., Given, K., \& Murrell, K. (2017). Systematic review of screening instruments for psychosocial problems in children and adolescents with long-term physical conditions. Global Pediatric Health, 4, 1-25. 
Thomas, D. R. (2017). Feedback from research participants: are member checks useful in qualitative research? Qualitative Research in Psychology, 14(23-41).

Tjønna, A. E., Stølen, T. O., Bye, A., Volden, M., Slørdahl, S. A., Odegård, R., ... Wisløff, U. (2009). Aerobic interval training reduces cardiovascular risk factors more than a multitreatment approach in overweight adolescents. Clinical Science, 116, 317-326.

Tomkinson, G. R., \& Olds, T. S. (2007). Secular changes in pediatric aerobic fitness test performance: the global picture. Medicine and Sports Science, 50, 46-66.

Townsend, N., Bhatnagar, P., Wickramasinghe, K., Williams, J., Vujcich, D., \& Rayner, M. (2013). Children and Young People Statistics 2013. London, UK.

Tracy, S. J. (2010). Qualitative quality: eight "big-tent" criteria for excellent qualitative research. Qualitative Inquiry, 16(10), 837-851.

Troiano, R. P., Berrigan, D., Dodd, K. W., Mâsse, L. C., Tilert, T., \& Mcdowell, M. (2008). Physical activity in the United States measured by accelerometer. Medicine and Science in Sports and Exercise, 40(1), 181-188.

Trost, S. G. (2007). Measurement of physical activity in children and adolescents. American Journal of Lifestyle Medicine, 1(4), 299-314.

Trost, S. G., Kerr, L. M., Ward, D. S., \& Pate, R. R. (2001). Physical activity and determinants of physical activity in obese and non-obese children. International Journal of Obesity and Related Metabolic Disorders, 25(6), 822-829.

Trost, S. G., Loprinzi, P. D., Moore, R., \& Pfeiffer, K. A. (2011). Comparison of accelerometer cut points for predicting activity intensity in youth. Medicine and Science in Sports and Exercise, 43(7), 1360-1368.

Truman, J., Robinson, K., Evans, A. L., Smith, D., Cunningham, L., Millward, R., \& Minnis, H. (2003). The Strengths and Difficulties Questionnaire: a pilot study of a new computer version of the self-report scale. European Child and Adolescent Psychiatry, 12(1), 9-14.

Tsutsumi, T., Don, B. M., Zaichkowsky, L. D., \& Delizonna, L. L. (1997). Physical fitness and psychological benefits of strength training in community dwelling older adults. Applied Human Science, 16(6), 257-266.

Ubel, P. A., Loewenstein, G., \& Jepson, C. (2003). Whose quality of life? A commentary exploring discrepancies between health state evaluations of patients and the general public. Quality of Life Research, 12(6), 599-607.

US Department of Health and Human Services. (1999). Mental health: a report by the Surgeon General. Rockville, MD.

Varey, C., \& Kahneman, D. (1992). Experiences extended across time: evaluation of moments and episodes. Journal of Behavioral Decision Making, 5(3), 169-185.

Varni, J. W., Burwinkle, T. M., \& Seid, M. (2006). The PedsQL ${ }^{\text {TM }} 4.0$ as a school population health measure: feasibility, reliability, and validity. Quality of Life Research, 15, 203-215.

Varni, J. W., Burwinkle, T. M., Seid, M., \& Skarr, D. (2003). The PedsQL ${ }^{\text {TM }} 4.0$ as a pediatric population health measure: feasibility, reliability, and validity. 
Ambulatory Pediatrics, 3(6), 329-341.

Varni, J. W., Seid, M., \& Rode, C. A. (1999). The PedsQL: measurement model for the pediatric quality of life inventory. Medical Care, 37(2), 126-139.

Varni, J. W., Seid, M. S., \& Kurtin, P. S. (2001). PedsQL ${ }^{\text {TM }} 4.0$ : reliability and validity of the Pediatric Quality of Life Inventory ${ }^{\mathrm{TM}}$ Version 4.0 generic core scales in healthy and patient populations. Medical Care, 39(8), 800-812.

Vlachioti, E., Matziou, V., Perdikaris, P., Mitsiou, M., Stylianou, C., Tsoumakas, K., $\&$ Moschovi, M. (2016). Assessment of quality of life of children and adolescents with cancer during their treatment. Japanese Journal of Clinical Oncology, 46(5), 453-461.

Vos, T., Barber, R. M., Bell, B., Bertozzi-Villa, A., Biryukov, S., Bolliger, I., ... Murray, C. J. L. (2015). Global, regional, and national incidence, prevalence, and years lived with disability for 301 acute and chronic diseases and injuries in 188 countries, 1990 - 2013: a systematic analysis for the Global Burden of Disease Study 2013. The Lancet, 386(9995), 743-800.

Wafa, S. W., Shahril, M. R., Ahmad, A., Zainuddin, L. R., Ismail, K. F., Aung, M. M. T., \& Yusoff, N. A. (2016). Association between physical activity and health-related quality of life in children: a cross-sectional study. Health and Quality of Life Outcomes, 14(71).

Wallander, J. L., \& Koot, H. M. (2016). Quality of life in children: a critical examination of concepts, approaches, issues, and future directions. Clinical Psychology Review, 45, 131-143.

Wang, Z. Y. (1999). Symptoms Checklist 90. In X. D. Wang, X. L. Wang, \& H. Ma (Eds.), Rating Scales for Mental Health (pp. 31-35). Beijing: Chinese Mental Health Journal Publishing Company.

Warburton, D. E. R., McKenzie, D. C., Haykowsky, M. J., Taylor, A., Shoemaker, P., Ignaszewski, A. P., \& Chan, S. Y. (2005). Effectiveness of high-intensity interval training for the rehabilitation of patients with coronary artery disease. The American Journal of Cardiology, 95(9), 1080-1084.

Warburton, D. E. R., Nicol, C. W., \& Bredin, S. S. D. (2006). Health benefits of physical activity: the evidence. Canadian Medical Association Journal, 174(6), 801-809.

Ware Jr, J. E., \& Sherbourne, C. D. (2015). The MOS 36-item short-form health survey (SF-36): I. Conceptual framework and item selection. Medical Care, 30(6), 473-483.

Warren, J. M., Henry, C. J. K., Lightowler, H. J., Bradshaw, S. M., \& Perwaiz, S. (2003). Evaluation of a pilot school programme aimed at the prevention of obesity in children. Health Promotion International, 18(4), 287-296.

Wasfy, M. M., \& Baggish, A. L. (2016). Exercise dose in clinical practice. Circulation, 133, 2297-2313.

Watson, D., Clark, L. A., \& Tellegen, A. (1988). Development and validation of brief measures of positive and negative affect: the PANAS scales. Journal of Personality and Social Psychology, 54(6), 1063-1070. 
Weinberg, R., Tenenbaum, G., McKenzie, A., Jackson, S., Anshel, M., Grove, R., \& Fogarty, G. (2000). Motivation for youth participation in sport and physical activity: relationships to culture, self-reported activity levels, and gender. International Journal of Sport Psychology, 31(3), 321-346.

Weissman, M. M., Orvaschel, H., \& Padian, N. (1980). Children's symptom and social functioning self-report scales: comparison of mothers' and children's reports. Journal of Nervous Mental Disorders, 168(12), 736-740.

Weisz, J. R., McCarty, C. A., \& Valeri, S. M. (2006). Effects of psychotherapy for depression in children and adolescents: A meta-analysis. Psychological Bulletin, 132(1), 132-149.

Welch, N., McNaughton, S. A., Hunter, W., Hume, C., \& Crawford, D. (2009). Is the perception of time pressure a barrier to healthy eating and physical activity among women? Public Health Nutrition, 12(7), 888-895.

Westgarth-Taylor, C., Hawley, J. A., Rickard, S., Myburgh, K. H., Noakes, T. D., \& Dennis, S. C. (1997). Metabolic and performance adaptations to interval training in endurance-trained cyclists. European Journal of Applied Physiology, 75(4), 298-304.

Weston, A. R., Myburgh, K. H., Lindsay, F. H., Dennis, S. C., Noakes, T. D., \& Hawley, J. A. (1997). Skeletal muscle buffering capacity and endurance performance after high-intensity interval training by well-trained cyclists. European Journal of Applied Physiology, 75(1), 7-13.

Weston, K. L., Azevedo, L. B., Bock, S., Weston, M., George, K. P., \& Batterham, A. M. (2016). Effect of novel, school-based high-intensity interval training (HIT) on cardiometabolic health in adolescents: Project FFAB (Fun Fast Activity Blasts) - an exploratory controlled before-and-after trial. PLoS ONE, 11(8), e0159116.

Weston, K. S., Wisløff, U., \& Coombes, J. S. (2014). High-intensity interval training in patients with lifestyle-induced cardiometabolic disease: a systematic review and meta-analysis. British Journal of Sports Medicine, 48(16), 1227-1234.

Weston, M., Taylor, K. L., Batterham, A. M., \& Hopkins, W. G. (2014). Effects of low-volume high-intensity interval training (HIT) on fitness in adults: a metaanalysis of controlled and non-controlled trials. Sports Medicine, 44, 10051017.

Whitehead, J. R., \& Corbin, C. B. (1991). Youth fitness testing: the effect of percentile-based evaulative feedback on intrinsic motivation. Research Quarterly for Exercise and Sport, 62(2), 225-231.

Whitehead, S., \& Biddle, S. (2008). Adolescent girls' perceptions of physical activity: a focus group study. European Physical Education Review, 14(2), 243262.

Whitelaw, S., Teuton, J., Swift, J., \& Scobie, G. (2010). The physical activity Mental wellbeing association in young people: a case study in dealing with a complex public health topic using a "realistic evaluation" framework. Mental Health and Physical Activity, 3(2), 61-66. 
Williams, C. A., \& Stevens, D. (2013). Physical activity and exercise training in young people with cystic fibrosis: current recommendations and evidence. Journal of Sport and Health Science, 2(1), 39-46.

Williams, D. M. (2008). Exercise, affect, and adherence: an integrated model and a case for self-paced exercise. Journal of Sport and Exercise Psychology, 30(5), 471-496.

Williams, J., Wake, M., Hesketh, K., Maher, E., \& Waters, E. (2005). Health-related quality of life of overweight and obese children. The Journal of the American Medical Association, 293(1), 70-76.

Wilson, I. B., \& Cleary, P. D. (1995). Linking clinical variables with health-related quality of life: a conceptual model of patient outcomes. The Journal of the American Medical Association, 273(1), 59-65.

Winn, C. O. N., Mackintosh, K. A., Eddolls, W. T. B., Stratton, G., Wilson, A. M., Rance, J. Y., ... Davies, G. A. (2017). Perceptions of asthma and exercise in adolescents with and without asthma. Journal of Asthma.

Wipfli, B. M., Rethorst, C. D., \& Landers, D. M. (2008). The anxiolytic effects of exercise: a meta-analysis of randomized trials and dose-response analysis. Journal of Sport and Exercise Psychology, 30(4), 392-410.

Wisløff, U., Ellingsen, Ø., \& Kemi, O. J. (2009). High-intensity interval training to maximize cardiac benefits of exercise training? Exercise and Sport Sciences Reviews, 37(3), 139-146.

Withall, J., Jago, R., \& Fox, K. R. (2011). Why some do but most don't. Barriers and enablers to engaging low-income groups in physical activity programmes: a mixed methods study. BMC Public Health, 11, 507.

Wong, N., Kady, L., Mewton, L., Sunderland, M., \& Andrews, G. (2014). Preventing anxiety and depression in adolescents: a randomised controlled trial of two school based internet-delivered cognitive behavioural therapy programmes. Internet Interventions, 1(2), 90-94.

World Health Organization. (2003). Caring for children and adolescents with mental disorders. Geneva, Switzerland.

World Health Organization. (2006). Constitution of the World Health Organization. Geneva, Switzerland.

World Health Organization. (2018). Strengthening mental health promotion (Fact sheet, No. 220). Geneva, Switzerland.

Wu, M. H., Lee, C. P., Hsu, S. C., Chang, C. M., \& Chen, C. Y. (2015). Effectiveness of high-intensity interval training on the mental and physical health of people with chronic schizophrenia. Neuropsychiatric Disease and Treatment, 11, 1255-1263.

Yu, S., Yarnell, J. W. G., Sweetnam, P. M., \& Murray, L. (2003). What level of physical activity protects against premature cardiovascular death? The Caerphilly study. Heart, 89(5), 502-506.

Zack, M. M. (2013). Health-Related Quality of Life - United States, 2006 and 2010. 
In CDC Health Disparities and Inequalities Report - United States, 2013 (Vol. 62, pp. 105-111). Atlanta, GA.

Zima, B. T., Murphy, J. M., Scholle, S. H., Hoagwood, K. E., Sachdeva, R. C., Mangione-Smith, R., ... Jellinek, M. (2013). National quality measures for child mental health care: background, progress, and next steps. Pediatrics, 131(Suppl 1), S38-S49.

Zuckerman, M., \& Lubin, B. (1990). Manual for the Multiple Affect Adjective Check List. San Diego, CA: Educational and Industrial Testing Service. 


\section{Appendices}

Appendix A - Eddolls, W. T. B., McNarry, M. A., Stratton, G., Winn, C. O. N., \& Mackintosh, K. A. (2017). High-intensity interval training interventions in children and adolescents: a systematic review. Sports Medicine, 47(11), 23632374

Sperts Med (2017) 47:2363-2374 DOI to. 10076+10279-017-0753-8 SYSTEMATIC REVIEW

High-Intensity Interval Training Interventions in Children and Adolescents: A Systematic Review

Wiliam T. B. Eddolls ${ }^{1}$ - Melitta A. MeNarry ${ }^{1}$ - Gareth Stratton ${ }^{1,2}$ -

Charles O. N. Wina - Kelly A. Mackintosh

Puttishat anline: 22 lane 2017

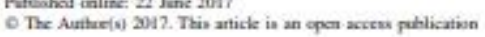

Ahstract

Background Whilst there is increacing interest in the efticacy of high-imensiay inserval training in children und adolescents as a time-effective method of eliciting heall benefins, there remains listle consenous within the literature regarding the most effective means for delivering a highinsensity interval training intervention. Given the globa health issoes swrounding childhood obesity and associated health implications, the idestification of effective inservention strategies is imperative.

Objectives The aim of this review was to examine thighinsensity imerval training as a means of influescing key health porameters and wo elucidate the most effective tighintensity iaterval training protocol

Malkads Studies were included if they: (1) studied healthy childrea and/or adokecents (ared 5-18 years); (2) poescribed an intervention that was deemed high inoensity; and (3) reported bealth-related outcome measures

Resuls A toeal of 2092 studies were initially retrieved from four databuses. Studies that were deemed to meet the crineria were doronlouded in their entirety and independently assessed for relevance by two authors using the pro-

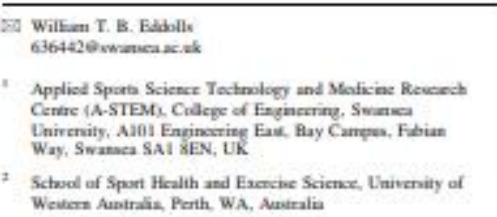

deterwined criveria. From this, 13 studies were deemed suisuble. This review foend that thigh-intensity interval training in children and adolescents is a time-effective method of improving candiovasceler disease biomarkers, but evidence regarding other health-related meaoures is nooer equivocal. Running-baved sessions, at an intensity of $390 \%$ beat rate maximum/ $100-130 \%$ maximal aerobic improvemens in participunt thealth.

Conclisvion While high-insensity interval training imperoves cardiovascular disease biomarkers, and the evidence supponts the effectiveness of running-based sessions, as coulined above, further recoumendations as to optimal exercise duration and red intervals remain ambigoosen owing to the pascity of literature and the methodological finitutions of sadies presently available.

\section{Ney Paints}

High-iwensity interval training cas improve certain cardiovascular health parameters in chaldren and adolescents.

Evidence supporting the overall effectiveness of high-intensity interval training as a means of eliciting improvements to other health coscomes. specibcally body composition and blood pressure, remains unclear.

While this review enables the estublishment of suggested guidelines for high-imensky interval training peotocols, recommendutions for souse protocol desails remain unclear. 


\section{Introductio}

Worldaide, the prevalence of childhood and adolescent obesity has reached unparalleled levels $[1,2]$. Specifically, in the UK, approximately $28 \%$ of childrea are classified as overweight or obese $[2,3]$, wepresenting a significant buroverweight or obese $\{2,3\}$, mepresenting a significant burden on health services. Indeed, current estimases saggest as type 2 diabeses mellitus [4] and coromary heart divease [5]. cost the Nixional Health Service f5.1 billion per year [6]. Whilst some reports suggest a plateau in paediatric [6]. Whilst some reports suggest a plateau in puediatric obesity over the last decade [7], others suggest that UK
obesity levels have increased between 1980 and 2014 by 48

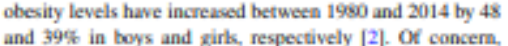
and $39 \%$ in boys and girk, respectively [2]. $\mathcal{O}$ concern, paediatric obesity has been wsocciated with an increased prevalence of candiometabolis risk factors [8], which the of premature morality $[9,10]$. Although the causes of obesity and cardiometabolic risk are multifaceted, low physical activity levels, as well as high engagement in sedentary pursaits, have been identified as key comonibutory factors $[9,11-13]$. Specifically, according to the lasest statistics, in England, only $21 \%$ of boys and $16 \%$ of girls meet UK physical activity guidelines of at least $60 \mathrm{~min}$ of moderate-to-vigoroes physicel activity every day [3], with physical activity with aqe $(14-16)$. Effective imerventions targeted at increasing youth physical activity levek are therefore impenaive.

While traditional interventions designed to increase physical activity and improve health have principally used moderate-intensity continuous exercise [17-19], the relevance of such programmes to the sporadic high-intensity nature of children's habitual play patterns has been questioned [20]. Comsequenuly, high-intensity imserval-based programmes have recently been investigated as a potentially posent and time-efficiemt form of physical activity and health promotion [21]. Indeed, whilst exercise, a subcomponeme of physical activity, is structured and conducted for the bealth-axsociated benefits, it could provide a necessary mediseory step to provoke positive long-term behuvioeral change. However, to date, studies have demonstrated varied saccess at eliciting significant improvements [22-25], posentially owing to a lack of consencous regarding in optimum high-intensity interval training (HIrT) intervention protocol with regard to exercise intensity, frequency and duration. Nonetheless, recent systematic reviews [26, 27] highlighted that HIIT can elicit greater improvements in healthelated porameers (ic. cardiometabolic health and body composition) in adolescents, compared with traditional programmes $[23,28-30]$. However, no systematic revieas have imvestigated the effects of HIIT on primary school-aged children, the identified potential differences between pre-pubertal and pubertal youth in the adapeations elicited of provided optimal HIIr proeocol recommendations. Therefore, the purpose of this review was to systematically synthesise the scientific literature reganding HIIT on improving body composition, candiometabolis health and candiavascular

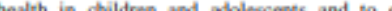
optimal HIIr prosocol with regard to session strocture. imsensity, frequency and duration.

\section{Methodology}

In line with the Preferred Reporting Items for Systematic Reviews and Meta-Analyses [31], the following method. ology details the review's inclusion criteria, search strah. egy, data collection and study analysis protocols.

\subsection{Inclusiou Criteria}

2.1.1 Types of Stoudy

The present review included studies involving interventions targesed at reducing obesity-relased ptrysiological parameters with a principal focus on one of the following: high-intensity persical activity, high-inechsity exercise/training of high-intensity intermittent/interval exercise/training interventions.

\subsubsection{Types of Participand}

Studies incooporating children and adolescents between the ages of 5 and 18 years were included. These age con-

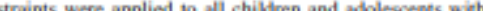
out disability, imespective of whether they were a healthy weighn, overweight of obese. Studies that wsed physical activity interventions as part of a treatment for specific illnesses were excloded.

2.1.3 Intenearion Variables and Oufcoms Measures

To be incladed in the review, studies were required to report a minimem of one intervention exercise sessice imsensity variable and one outcome measure, meavured at baseline and post-intervention and compured with either a moderate-intensity exercise intervention or control group.

2.1.4 Intervention Materesiry Variables

Interventions were defined as high intensity if: (1) the incensity was $\geq 90 \%$ peak axygen uptake [32]: (2) had an insensity that was $\geq 100 \%$ maximal aerobic speed [33]; and/or (3) ensured that the participant's heart rate was

Q Springer 
$\geq 90 \%$ of their peak heart rate [34, 35]. There were no restaictions applied regarding the daration of the intervention.

\subsubsection{Primary Ounows}

Primary outcomes included cardiometabolic health markens, namely mean systolic (SBP) and diastolic (DBP) bloow pressure, body composition in the form of body mass inde: (BMII), body fat percentage (BPF) and fat-tree max, and cardiovascular disease (CVD) biomarker analysis including at least one of the following: glucose, insulin, triglycerid: and total cholesterol, as well as its sub-fractions, lowdensity lipoprosein cholesterol and high-density lipoproetin cholesterol.

\subsection{Search Strategy}

Electronic dacabases were searched until September 2016, with no restriction set an the publication year. The PubMed and SCOPUS databases were explored uxing the following keyword search strategy, devised by the research team and verified by a subject libraian: (high intemsity training $\mathrm{OB}$ high intensity exercise* OR high intensity activit* OR high insensity intermittent training $O R$ intensity intermittent exercise* $\mathrm{OR}$ high imensity imermiktent activit* or high imsensity interval training $O R$ high intensity interval exercise" OR high intensity interval activis*) AND (child* $\mathrm{O}$ children $\mathrm{OR}$ pediatric $\mathrm{OR}$ paediatric $\mathrm{OR}$ adolescen* $\mathrm{O}$ juvenile*) AND (health OR healthy). Inclusion of at least one of the keywords was required in the study title for it to be considered. Studies were excluded based on language only stodies written in English were included. Addition: stadies were identifed by searching the reference lists of included studies. Google Scholar and Reseanchlate wer: also searched to identify studies that were potentially overlooked by the database searches

\subsection{Data Collection and Analysk}

Pertinent stady abstracts from the stated search strategies were downloaded and independently screened. Stodes th were deemed to meet the criteria were downloaded in their entirety and independenaly assessed for relevance by two authors waing the pre-determined criteri. When stady information was missing, the research team attempted to consact the primary unthoe of the incomplete study. If the author failed to respond, the study was excluded.

\subsection{Effect Size}

Coben's $d$ was weed wo desermine the standardised mean effect of HIIT on the previously outlined bealth-related outcome measures companed with a control group or a moderate- or light-intensity group [36]. Confidence intervals (Cls) were calculated by applying an ecuation recoummended by Nakagawa and Cuthill [37], employing standard error calculations [38]. For stadies that provided values for both moderate and control groups, moderise

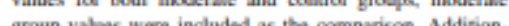

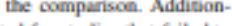
ally, effect size was not calculated for stadies that failed to disclose post-intervention mean values.

\subsection{Risk of Bias Assessment}

Risk of bias was assessed independendly by two revieaers using the Cochrane risk of bias tool (RoB 20) [39]. Using the RoB $2.0 \mathrm{tool}$, stodies were awarded an overall risk of bias grade of either high, some or low risk of bias. This overall grade was calculated by asessing five domains: (A) bias arising from the randomisation process; (B) bias owing to devixions from intended interventions; (C) bias owing to missing outoome data; (D) bias in measurement of the outcome; and (E) bias in selection of the reported resalt.

\subsection{Heterogeneity Assessment}

Because of the variation of the study characteristics in this review, for example between interventions, outcome mea. sures and cohort popolations, it was deemed unsuitable to amalgamate the resuls for a meta-analysis. Therefore, the resalks in this review were analysed narratively.

\section{Results}

The database search generaied 2092 studies. Once dupli. cates were removed, 54 title/abotracts were screened for eligibaily, with the reference list search producing three further studies. From this, 13 studies were deemed suitable. The screening process is shown in Fig. 1. In total, 7 of the 13 studies included in this review examined the effects of HIIT innerventions on pre-pubertal participants, the characteristics of athich are sammarised in Table 1 , with the remaining six stodies exansining the effects of HWT interventions on pubertal participants (Table 2).

\subsection{Risk of Bias}

The methodological rigour of stadies incloded in this review, according to the ridk of thias assessment, is presented in Table 3. Seven stodies were considered to have a high rikk of bias [22, 24, 25, 29, 40,41, 45]. whereas only two $[28,43]$ and for $[23,30,42,44]$ studies were contwo $[28,43]$ and for $[23,30,42,44]$ stodies were con- 
Fig. 1 Phanes of stud

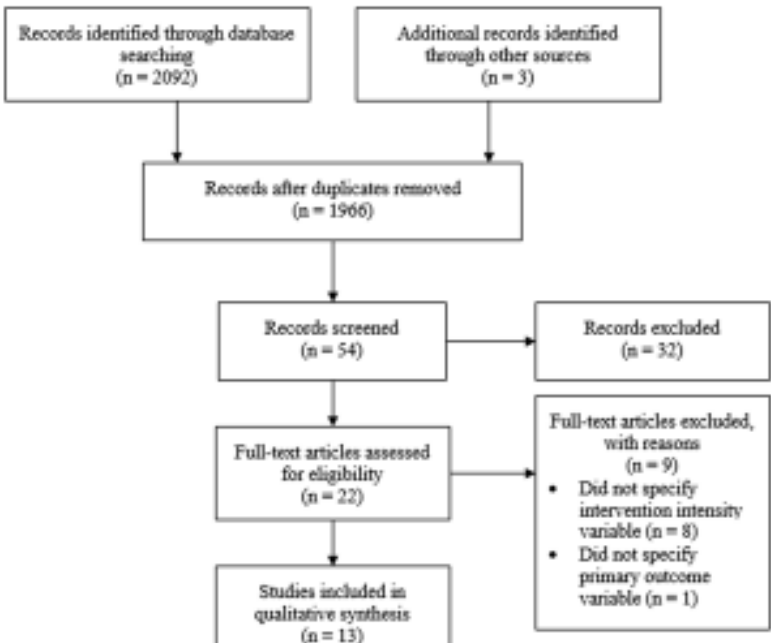

stodies deemed to have a high or some risk of bias $[22,24,25,28,29,40,41,43,45]$, the bias arose from the randomisation process (domain $\mathbf{A}$ ).

\subsection{Bedy Mass and Compesition}

All 13 included studies reponted the effect of HIIr com pared with moderate-iniensity exercike or a control groep on BMI $(n=9)$, BP The results revealed little evidence to suggest that HUT ce elicit significant changes in body composition (Table 4), although Tjonna et al. [23] and Racil et al. [30], boch of which were assessed to be at low risk of bias, reporied significant improvements in BMII and BP\% associaned with a medium-to-large effect sizes following a 3-mont inservention.

It is pertinent to note that whilst other stodies failed to find a significant imporovement in measures of body mass or composition, there was a general trend for a greater change in body mass and composition in the HIrT groeg $[22,28,29,43,45]$. The exception to this was Baquet et al. [25], whe saw significant increases to BMI and BP\% for both the HIIT protocol and the control group, however, this stady was deemed to have a high risk of bias. While mo significant benefins were reperted in pre-pubernal children significant improvements in body mass and composition have been demowstrated in pubertal children $[23,30]$. suggesting a poeential maturational effect. There were inoufficient data to examine potential sex differences reganding the effscacy of HIIT in eliciting significant changes in body composition.

\subsection{Cardievascular Healt!}

3.3.1 Blood Pressure

Five studies investigated the effect of HIIT on SBP and DBP, with the majocity concluding significant benefits were obained (Table 5). Specifically, two studies that were assessed to be of low ratk of bias. Tionm et al. [23] and Racil et a. [30], reported significant improvements in both SBP and DBP following the intervention, although it is pertinent to mooe the low effect sizes associated with the (30i) (Table 5). Whitat the ren trend towards a lower SBP and DBP. Interestingly, Boddy et al. [44], demonotrating methodological rigour through a low risk of bias, found an increase in both DBP and SBP in the HIIT group and reductions in the control group, thoogh not signibcark. Regarding maturation differences, to stodies examining pre-pubertal children reported 


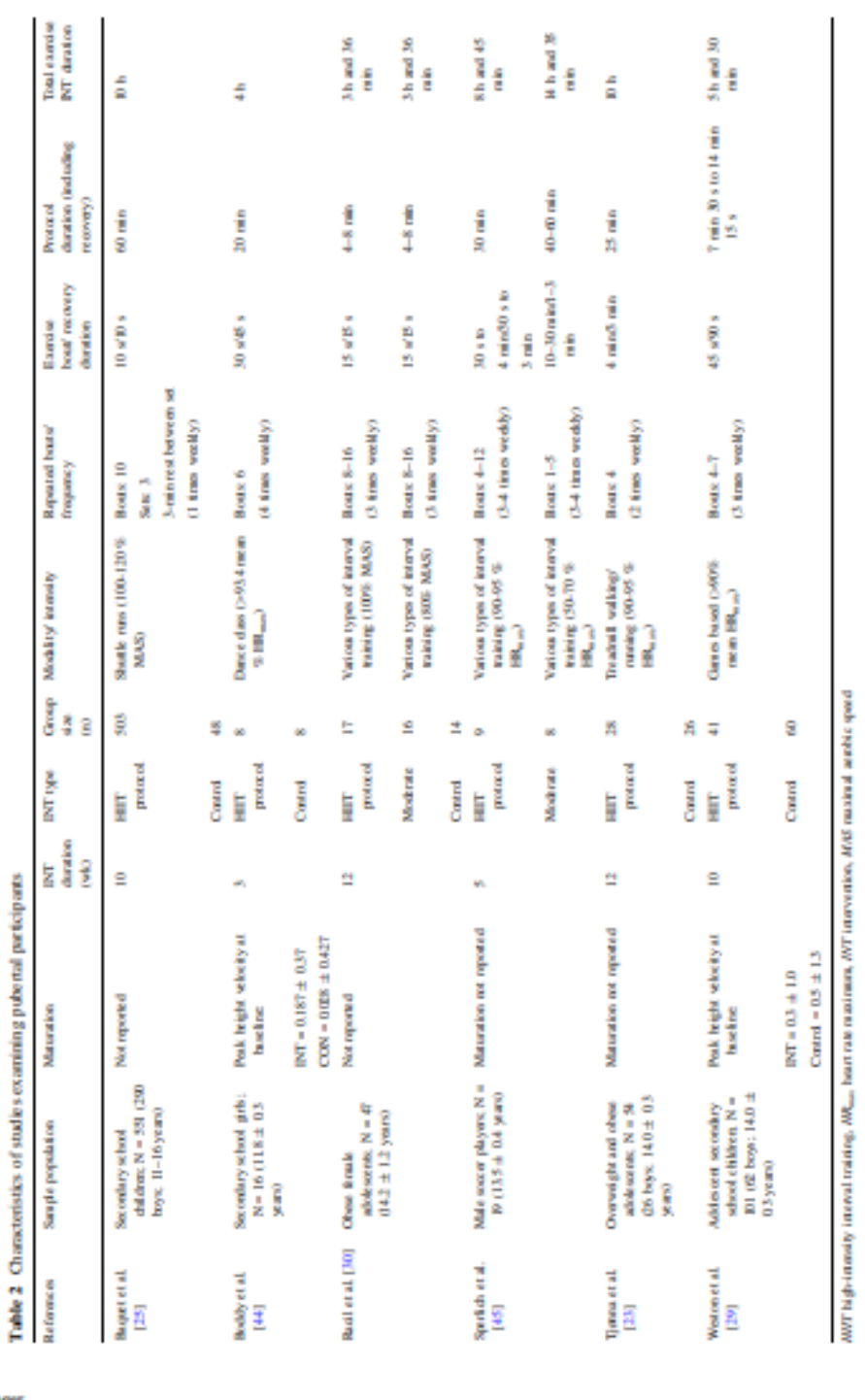

Q springer 
Tahle 3 Rick of bis mosment

\begin{tabular}{|c|c|c|c|c|c|c|}
\hline Referentes: & Derrain A & Demain B & Denain C & Damia D & Damain E & Toted \\
\hline Baquat et al. [22] & High & Lew & Low & Low & Lum & High \\
\hline Baquat et al. [25] & Hegh & Lest & Low & Low & Lan & High \\
\hline 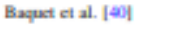 & $\mathrm{Hg}_{\mathrm{gh}}$ & Low & Low & Lanw & Lurw & Fight \\
\hline Baquat et al. [41] & Higk & Los & Low & Lum & Low & Hight \\
\hline Boddy at al [44] & Low & Lest & Low & Lum & Larw & Low \\
\hline Lenbrick et al. $\mid 42]$ & Low & Low & Low & Low & Lane & Lenw \\
\hline Las at al. [24] & High & Low & Low & Low & Lanw & Hight \\
\hline Nourry et al. [43] & Sunec & Len & Low & Low & Low & Same \\
\hline Racil et al. [30\}] & Low & Low & Low & Lum & Lan & Lew \\
\hline Reosenkranz of al [2X] & Sunec & Lew & Low & Low & Lan & Same \\
\hline Sperlisish et al. [45] & Hegh & Low & Low & Lumw & Low & High \\
\hline Tytana et al. [23] & Low & Low & Low & Lunw & Lan & Lonw \\
\hline Wotre et al. [29] & Hght & Low & Low & Lurw & Lurw & High \\
\hline
\end{tabular}

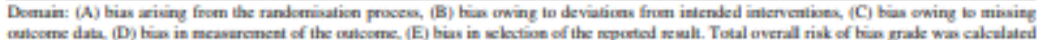

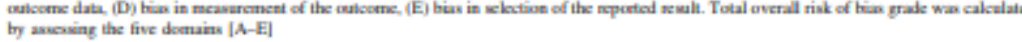

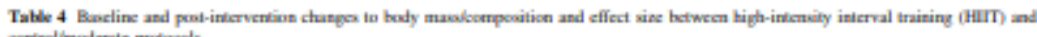
sontinolimoderate protiosol

\begin{tabular}{|c|c|c|c|c|c|}
\hline Resinences: & Orticume meavers & $\begin{array}{l}\text { HIIT (mean change } \\
\text { frour busline) }\end{array}$ & 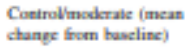 & $\begin{array}{l}\text { Effeat är } \\
\text { (Cohan's d) }\end{array}$ & $25 \% \mathrm{C}$ \\
\hline Baypect of al. [25] & BMI $\left(\mathrm{kg} / \mathrm{m}^{2}\right)$ & $0.40^{* *}$ & $0.60=0$ & -0.14 & -0.78 to 0.49 \\
\hline Boyect if al. [40] & BMI $\left(\mathrm{kg}^{2} \mathrm{~m}^{2}\right)$ & 0.10 & -0.300 .20 & -0.10 & -0.78 to 0.58 \\
\hline Boddy et al [44] & BMI $\left(\mathrm{kg}^{2} \mathrm{~m}^{2}\right)$ & -0.50 & 0.20 & a.93 & $0.30 \mathrm{w} 1.56$ \\
\hline Lambrick of al. $\mid 42\}$ & BMI $\left(\mathrm{kg}^{2} \mathrm{~m}^{2}\right)$ & 0.00 & 0.00 & a.23 & -0.20 to 0.87 \\
\hline Law el at. [24] & BMI $\left(\mathrm{kg} / \mathrm{m}^{2}\right)$ & 0.20 & $0.100 .40^{*}$ & 0.42 & -0.18 to 1.01 \\
\hline Raxil at al [30] & BMI $\left(\mathrm{kg} / \mathrm{m}^{2}\right)$ & $-3.20^{\circ}$ & $0.30 y=1.70^{\circ}$ & -1.41 & -3.52 to 0.69 \\
\hline Rouscrikrane et al. [28] & BMI $\left(\mathrm{kg}^{2} / \mathrm{m}^{2}\right)$ & -1.40 & 0.00 & -0.06 & -0.73 to 0.61 \\
\hline Tjensa af al. [23] & BMI $\left(\mathrm{kg}^{\mathrm{m}} \mathrm{m}^{2}\right)$ & $-0.70^{* *}$ & -0.20 & -1.50 & -206 to -0.94 \\
\hline Weston et al. [29] & BMI $\left(\mathrm{kg}^{2} \mathrm{~m}^{2}\right)$ & $-0.000^{\circ}$ & essr & N/A & N/A \\
\hline Baypect if al. [25] & Boty fat (w) & $1.60^{\circ}$ & $1.30^{\circ}$ & -0.31 & -0.94 is 0.33 \\
\hline Boypect at al. [2] & Bodty $f=(\omega)$ & -0.90 & -0.70 & Q.10 & -0.51 to 0.71 \\
\hline Baypect ef al. [41] & Baty fat (w) & 0.10 & Q.10 & $\omega \omega 2$ & -066 to 0.69 \\
\hline Boddy et al [44] & Bacty fat (W) & 0.22 & 0.46 & 0.61 & $0.14=1.07$ \\
\hline Lambrick of al. [42] & Bady $f=(\%)$ & -0.10 & 0.40 & $a c s$ & -0.29 t 0.65 \\
\hline Nourry at al. [43] & Bady $f:(\Phi)$ & 150 & aso & 0.14 & -0.56 to 0.54 \\
\hline Rasil at al [30] & Bady $f=(\omega)$ & $-3.90^{*}$ & $=0.50^{-1}-3.20 \%$ & -0.59 & -1.06 to $=0.12$ \\
\hline Resecrikrane et al. [28] & Bady fat (w) & -2.20 & -1.00 & -0.17 & -0.80 to 6.45 \\
\hline Tjonsa ef al. [23] & Bady $f=(\omega)$ & $-0.90^{* *}$ & -0.30 & 3.00 & 2.3563 .65 \\
\hline Spertich et al. [05] & FFM (bz) & 1.00 & a.90 & a.71 & 0.28 to 1.13 \\
\hline
\end{tabular}

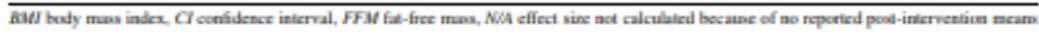

$* p<0.05, * p<0$ 0.01, sipnificantly different from buscline

- Values adjantod fer nex, bueline value and maturity offect

significant changes, whereas significant improvements were reported in pubertal popalations $[23,30\}$. Sex dit-

terences could not be investigated because of insufficient data.

3.3.2 Cardionascular Disnase Biowasker Health

Foor studies examined the effect of HIIT on CVD

biomarkers, specifically blood glocose $(n=4)$, toeal

Q springer 


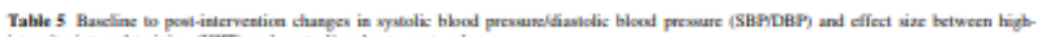

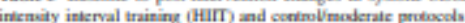

\begin{tabular}{|c|c|c|c|c|c|}
\hline Beferentres: & $\begin{array}{l}\text { Ouloseme measure } \\
\text { (mmily) }\end{array}$ & $\begin{array}{l}\text { HIIT (mean charge } \\
\text { from buseline) }\end{array}$ & $\begin{array}{l}\text { Conitrul/moderate (mean } \\
\text { change frem bueline) }\end{array}$ & $\begin{array}{l}\text { Effect sise } \\
\text { (Coben'x } d \text { ) }\end{array}$ & $95 \% \mathrm{C}$ \\
\hline Boddy at al [44] & SRP & 10.10 & -1.40 & 0.34 & 0.08 to 0.61 \\
\hline Raxil et al. [39| & SAP & $-0.60^{\circ}$ & $0.00 y-0.40^{*}$ & $a, 00$ & -0.26 to 0.26 \\
\hline Resecrikrass of al [28] & SRP & -2.20 & -2.50 & -0.54 & $-0.52 \mathrm{de}-0.2 \mathrm{t}$ \\
\hline Tyena et al. [23] & SBP & $-9.40^{* *}$ & $-2.50^{\circ}$ & -200 & $-2.31 \mathrm{db}-1.60$ \\
\hline Wotue at al [20] & SBP & $-5.00 r$ & $-1.00^{2}$ & $\mathrm{NA}$ & NA \\
\hline Boddy at al [44] & DEP & 5.90 & -4.10 & 1.14 & 0.75 t 1.52 \\
\hline Racil et al. [39] & DBP & $-600^{*}$ & $-1.0 y-4.00 \%$ & -0.32 & -0.64 to 0.01 \\
\hline Rlosenkranz of al [2X] & DEP & -2.50 & -1.70 & -0.83 & $-1.18 \mathrm{de}-0.48$ \\
\hline Tyena et al. [23] & DBP & $-5.50^{* *}$ & 1.80 & -1.50 & $-1.50 \mathrm{do}-1.11$ \\
\hline Wotun at al [29] & DBP & $-600 r$ & $-400^{\circ}$ & NA & $\mathrm{N} / \mathrm{A}$ \\
\hline
\end{tabular}

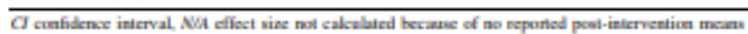

$* p<0.05 ; * t p<0.04$ significatly different frem bacline

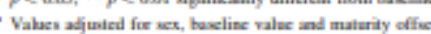

cholesterol ( $n=2$ ), hizh-density lipoprotein cholesterol $(n-3)$, low-density lipopeotein cholesterol $(n-1)$ ), blood triglycerides $(n=3)$ and insulin $(n=2)$. The results, in Table 6, suppon HIIT as an effective strategy for mproving CVD biomarker health. All $\alpha$ the stodies demonstrated significant $[23,28,30]$ or clinically substantial [29] improvements in multiple CVD biomarker

outcome measures. Tjanna et al. [23] reported greater significant improvements for blood zlucose in favour of the HIIT group $(p<0.01)$ compared with the control groep $(p<0.05)$, with an effect size indicating large clinically mportant differences $(d=-1.43,95 \% \mathrm{Cl}-3.0100 .16)$. Additionally, results from Rosenkranx et al. [28] saggest arge signiscant redoctions in total cholesterol compared

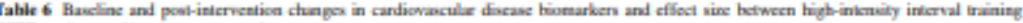
(HIIT) and cuetrolinoderais protinsobi

\begin{tabular}{|c|c|c|c|c|c|}
\hline Refefernas: & Oulcome meaure & $\begin{array}{l}\text { HIIT (mean change } \\
\text { from baselase) }\end{array}$ & $\begin{array}{l}\text { Controlimoderaile (mean } \\
\text { change frum buacline) }\end{array}$ & $\begin{array}{l}\text { Eifect sine } \\
\text { (Cuhen'x } d \text { ) }\end{array}$ & $95 \% \mathrm{CI}$ \\
\hline Racil et al. [\$S] & Cluxuese (mmole $\left.\mathrm{L}^{-1}\right)$ & $-0.20^{*}$ & $0.00-0.200$ & -0.32 & -0.4 is 0.13 \\
\hline Resenkrans of al [2X] & Gluxues (mejtL) & 5.20 & an & -0.16 & -1.80 to 1.17 \\
\hline Tyena et al. [23] & 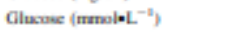 & $-0.30 *$ & -0.10 & $-1,43$ & -301 to 0.16 \\
\hline Wotra et al [29] & Claxues $\left(\operatorname{manol} \cdot \mathrm{L}^{-1}\right)$ & $-0.10 \%$ & $-0.03^{*}$ & $\mathrm{~N} / \mathrm{A}$ & $\mathrm{NA}$ \\
\hline Resertibrass of al [2X] & Total chalesterol (mg/AL) & $-2200 \%$ & $2 \omega 0$ & -0.93 & $-1.16 \mathrm{~b}-0.30$ \\
\hline Wotud et al [29] & Total chulesderul (mmalvel. ${ }^{-1}$ ) & $-0.2 \sigma^{2}$ & $00 \sigma^{2}$ & N/A & NA \\
\hline Rosentrans of al [2X] & HDL-dholestend (mplit.) & 9.90 & 360 & 0.42 & 002 to 0.81 \\
\hline Tyena et al. [23] & HDL-Cholestend (mmalkL. ${ }^{-1}$ ) & $0.11^{*}$ & $0 \omega$ & 0.35 & -0.50 to 1.20 \\
\hline Wotus et al [29] & 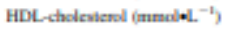 & -0.14 & $-0.24^{*}$ & $\mathrm{~N} / \mathrm{A}$ & NA \\
\hline Resentrans of al [28] & LX-chrlaternd (mg/AL) & $-34 \mathrm{Non}$ & -5.60 & -1.67 & $-2.03 \mathrm{~b}-1.31$ \\
\hline Resenkrane et al. $(2012)$ [28] & Triglyctrides (mgdL) & 23.50 & 3.50 & $-\infty .07$ & -0.33 to 0.19 \\
\hline Typra et al. [23] & 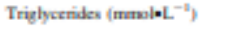 & -0.50 & -0.10 & -0.71 & -1.57 tง 0.14 \\
\hline Wotun et at. [29] & Triglyzrrides (mavi $\mid \mathrm{L}^{-4}$ ) & $-0.06^{\circ}$ & $0.18^{\circ}$ & N/A & NA \\
\hline Racil et al. [30] & Invalin $\left(\mathrm{GU} \mathbf{m L} \mathbf{L}^{-1}\right)$ & $-5.70^{*}$ & $-0.80-4.30^{\circ}$ & $-a \times 2$ & $-1.55 b-0.10$ \\
\hline Tyma et al. [23] & Insulin (pmolL.) & $-54.30^{\circ}$ & $-33.60^{\circ}$ & -0.46 & $-0.20 \mathrm{~b}-a .22$ \\
\hline
\end{tabular}

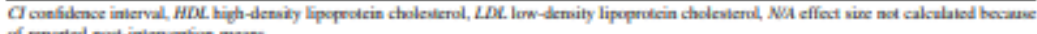

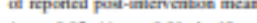

$* p<0.05 ; * p<0.04$ significantly different from baxline

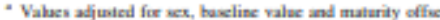

\section{Q spriager}


with the control groep. Further, the aswociated effect size (d $=-0.93$, 95\% CI -1.16 to -0.70$)$ suggested highly clinically important differences. Rosenkranz et al. [28] saw significant reductions $(p<0.08)$ in low-density lipoproeei cholesterol as a result of HIrr, with a large and clinically important difference $(d--1.67 .95 \% \mathrm{Cl}-2.03$ mo -1.31$)$ compared with a small non-signifcant increase in the control groop. Examining blood insulin results in Tjonn et al. [23], larger significant redactions in the HIIT groop were found compared with the control group, with further significant redactices after a 12-month follow-up. I addition, high-density lipoprosein cholesterol significantly improved in the HIIT group compared with a non-significant increase in the control group. Furthermoore, Racil et al. [30) reporied signibic insalin in boh HIIr and moderate-intensity probocols compared with a coetrol group, with a greaner improvement reported in the HIIT groep for both measures. Effect sizes for boh blood glucose and insulin were bow to modernet $(d=0.32,95 \% \mathrm{Cl}=0.44$ w0.13) and large $(d=-0.82$. $95 \% \mathrm{Cl}=1.55$ to -0.10 , respectively.

Notwithotanding the limised improvement in blood triglycerides, Weston et al. [29] reported clinically substantial beneficial effects as a resalt of HIIT despite increased triglyceride levels reponted in the control group. Despin these encouraging findings, it is pertinent to note that the stodies by Rosenkranz et al. [28] and Weston et al. [29] were assessed to have some and a high risk of bias, respectively. therefore, caution should he talen when iaterpreting these stodies as method stadies as method the results. There was no effect owing 00 maturation on CVD biomarkers, with significant or clinically substantid imporovements found in both pre-pubertal [28] and puberti $[23,29,30]$ children. Sex differences were nos reponted and therefore, their effect is unknown.

\subsection{High-Inteasity Interval Training Intervention Protocal}

All studies included in this review provided a detaile description of their intervention protocol in terms of sessice structurne, duration, intensity and frequency, in addition to intervention duration key details of these are summarised in Tables 1 and 2. Based on the foor stodes that demonstrated significant health improvements $[23,28-30]$. a runningbased HIIr inservention at an intensily of $>90 \%$ heart rate maximum/100-130 \% maximal aerobic velocity, two wo tree times a week wath a minimum inservention daratice lasting 7 weels could be considered the seygested practice. However, suggested exercise session duration and rest studies, a motion also sapponed by Baquet et al. [46].

4 Discussion

The aim of the current review was to symesise previous thenaure that examined HIIT in children and adolescents and establish its porential effect on body composition, cardiometabolic health and cardiovascular health. In abdition, this review aimed to identify an opeimsal HIIT protocol with regand to session strecture, intensity, frequency and duration. In accond with this aim 13 stodies were evaluated providing evidence suggesting that HIIr can significantly mprove certain bealth parameters in children and adolescents. However, evidence supporting the overall effectiveness of HIIT as a means of eliciting improvements to

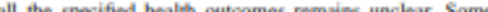
guidelines for a HIIT protocol were established, thoagh recommendations for certain protocol detaik remain unidentified.

Advancing previous reviews [26, 27], the findings of the curremt review sagest that pubertal children may achieve a greater benefit as a resalt of HIIT when compared with prepubertal children, a topic that has been widely debated (47-Soj. However, is is pertinent w note that this may be 2 the imerpretation of previoes studies. First, the duration of the HIIT interventions examining pubertal participants tended to be longer than those in pre-pubertal children. Given the present findings sargesting that a minimum of 7 weeks is required for siznificant adapeations to be man-

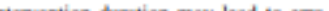

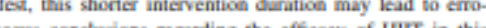
of HIIr in this population.

Furthermore, differences in the participant characteristics between pre-pubertal and pabertal stodies with regand to baseline body mass or body composition and health status may confound interpretation of inter-study differences and their attribution to maturity per se. Specifically, the majority of studies in pre-pubertal children used those of a moemal weight compared with the inclasion of overweight or obese participants in perbertal studies, which may predispose these latter studies to demonstrating greater health benefits, imespective of their biological age. Moreover, both Tjomna et al. [23] and Racil et al. [30] did not report maturation stages, subsequently casting ambiguity wer the cobort's troe maturecional stage. Despite this, when focusing on the additional stady involving pubertal children [29], is generally elicited greater improvemens in outcome measures when compared with the stody imvolving pre-pubertul children [28]. Moreover, only two of the stodies involving pubertal children that demonstrated positive signifcan results $[23,30]$ conoidered dietary intake; a bilure to acoount for changes in chetary' intalk

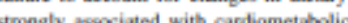


Desity [52] in children and adolescents, confounds the inserpretation of the results and their atribution to the exercise per se. Finally, in addition to the aforementioned methodological limitations, the interpretation of the overal findings of this study may also be limited by a mixed risk of bias between the significant studies.

An adidienal facter tat may ooenribute to the collestive umbiguity megud astion 'compensation effect'. Specifically, a recem study has suggested that school children appear to compensate for increased physical activity levels, with a redaction in physical activity undertaken the folloring day [53]. Additionally, the 'uctivinystat' bypochesis suzgests tha screased levels of physical activity during coe part of the day may result in a compensutory decrease in physica activity in another part [54]. Therefore, there is scope to suggest that prescribing exescise that reflects the charac teristics of children's comparatively elevated levels of hatitual play [55] may result in a decrease in habitu. physical activiny levels that day or the suboequent day. This therefore highlights the need to measure habitual physical activity, as was the case for only foor of the stodies included within this review $[23,28,29,44]$, alongside the previoesaly outlined study outcomes. Regarding the effect o HIIT between sexes, no cosclasions could be drawn none of the studies included in this review provided breakdown of between sex differences. This coeld have mplications for fisure reseanch, given the possibility the eflects of exercise interventioes on body sine [56, 57], cardiorespiratory health [58] and cardiometabolic health 157) may be sex dependent in children. Therefore, future cuadies stould endeavour to report a more expansive breakdown of results, thus providing clarification as to possible sex and maxurational differences associated wit HIIT.

Whilst the studies within this review have advanced ou understanding rezarding the influence of HIIT in childre under laboratory-based conditions, the relatively small sample sizes and intervention delivery methods highlight potential issues regarding larger scale implementation of HIIT. Schools have frequently been wed as a foundation in the implementation of physical activity interventions $59,60]$ because of their access to a greater population of

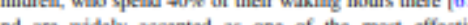
wocations to promote physical activity and health. Furhermore, previows studies have demoenstrated how shorlerm HIIT interventions have been successfully embedded within the school timetable $[44,62]$. It has been suggested that HIIT can allow foe greater class control compured with comentional phycical edueation lessons and ean be capted to inclate specife movements related to different ports [63]. Therefore, siven the promising findings regarding the effectiveness of HIrr, future research may wish to consider bow long-term HIrr interventions could be incorporated within the school environment. A key consideration in the development of future interventions, and purticipans'st engasement in and adterence to the Whilst HIIr has teen suggeded to be a preferable exercise modality to more conventional aerobic exercise [26], further research is required, with only one study in the present review considering this aspect of intervention development and implementation [42]. Finally, the sustained post-intervention efficacy of HIIT imerventions that reported siznificant improvements to body composition and car-

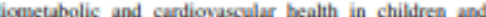
adolescents remains indeterminate owing to a predominant scarcity of studies reporting a post-intervention follow-up. Therefore, future studies stowald incorporate a follow-up period within their study design to assess the long-term post-intervention sustainabiliny of positive HIIT elicited benefins.

\section{Condusion}

High-intensity inserval training is a time-effective method of improving CVD biomarker health in children and adolescents. Horwever, evidence supporting is effectiveness in additional health measures remains equivocal. This review suggests that running-bused sessicens, at an intensity of

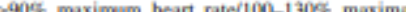
Jake times a week and with a minimums imservention duration lasting $>7$ wecks, elicit improvements in health makers bowever, these findings are limised by the mixed risk of bias between the significant stodies. Further recommendations as to exercise duration and rest intervals remain ambigwoes owing to the pascity and methodological limitations of studies presently available.

Complance with Ethical Standard

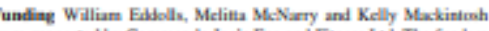
were upperted by Conenando bec'x Fun and Fitsex Lid. The funder

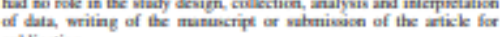
publication.

Conflict of hebrest walliam Eddolke Melita MeNarry, Careth

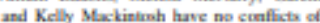

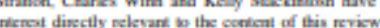

Batios approval Apporval to cunduct this nescench war granted by 
Open Access This anticle is daitributed under the lerms of the

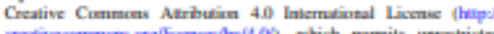

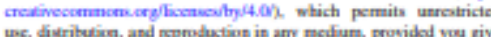

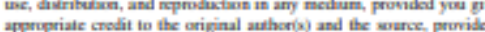
link to the Creative Commea liceme, and indicate if ctuages were made.

\section{References}

1. Letedcin T, Jaskwe-Leach R, Moodie MEl, et al. Child and adrlacent docity: part of a bigger pichure. Lascet.
2015:385-2510-20.

2. Ng M. Fleming T, Redinese M, et al. Chobal, regicend, and sational preralesce of onerweigtt and obesily in chilinen and

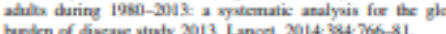

3. Ryklen of divenes study 2013. Lancel 2014:34:766-81.

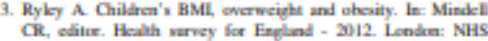
OR, oditice. Hest

4. Anerican Debeter Aassciatica. Type 2 dibctes in chülren and

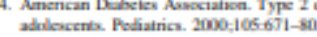

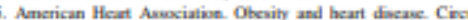

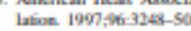

6. Sarbersugh $\mathrm{P}$, Bhatrager $\mathrm{P}$, Wiskramairghe KK, et al. The occovereic berden of ill heilth due to det, phyaical insativity, senching, aloutal and obenily in the UK: an updte to 2006

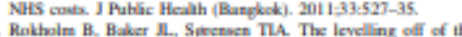

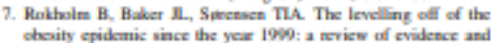
chosity epidanic nince the yese 1900: 1

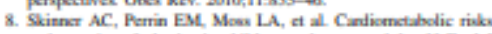

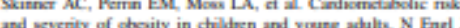
Mod. 2015;373:1307-17.

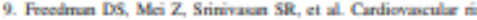
factoes and eacres actpen adrlocerts: the Bogaluas Heat Sudy. I Pediat.

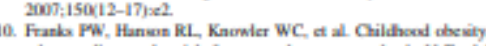

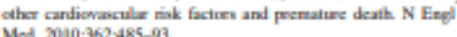

11. Janoen I, Katmersyk PT, Boyce WF, at al. Compariwe of oncrweight and abecily prevalence in schod-agod youth frum 34 petteme Otos Rev. 2005, 1 123-32

12. Trut SQ Kerr LM, Ward DS, et al. Promeal activity and

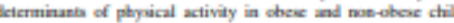
dren. Int I Obes Relat Metah Diend. 2001;25:822-9

13. Kupelintar PO. Otesity a a meitad problem Natur:

14. Sills IF, Proctuba II, Tagles WC. A neview of comrelutes of phrosial activity of children and adolewente. Med Sci Spourt

5. Heirlar C, Lyte L, Eriskwe D et al. Phycical activity and

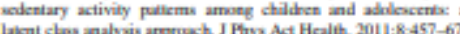

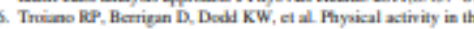

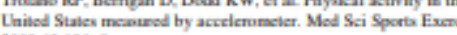
2008:40: $181-8$.

17. Mcrurray RQ, Harrell IS, Bangliwala S1, et al. A school-troed

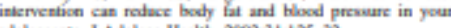

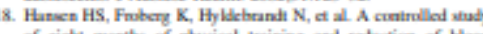
of cight marths of plysical trairing and redection of thood

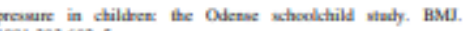
$203652-5$

9. Meyer AA Kundt G, Lenshow U, at al. Imprenement of early

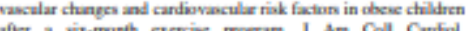
after as acomint

20. Bailey RC, Obua I. Popper SL, et al The level and iterpo of

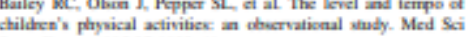

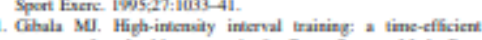

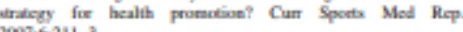
Bepect $\mathrm{C}, \mathrm{B}$

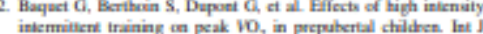
Sports Med. 2002;23:439-44.

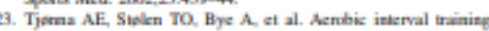

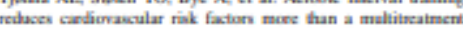

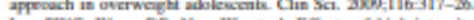
Las PAC, Wang De, Ngo JK, at al. Elfext of thigh-intenaity

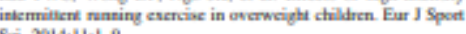

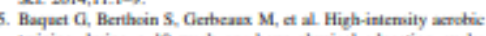

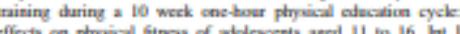
effect on phymad finos of

6. Logan CRM, Heris N, Dunese S, at al. A reries of adrlocent tigh-irtcnsity interval traizing, Spert Med. 2014;44:1071-85.

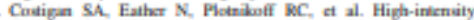
interval training for introving healh-relatod fitros in adobs.

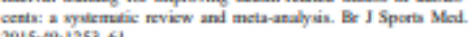

8. Rusenkrane SK, Rusenkrane RR, Hawnann TI, et al High-ie-

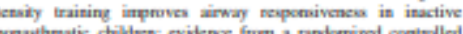
trial I A Apd Froviol. 2012:112:1174-83.

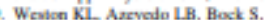

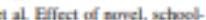

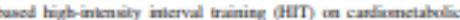

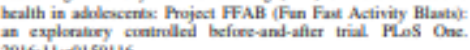
$2016: 11$ : 00159116

0. Racil G, Coypart IB, Elmontawe W, et al. Greatcr effect of

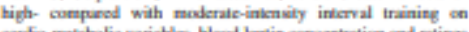

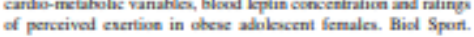
of perctived esaction

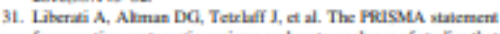

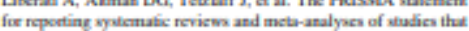
evaluts healh care interverticase explaration and elaboration.

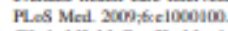

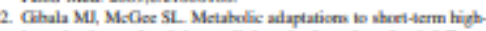

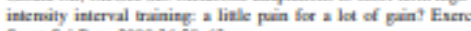
Sport Si Rev. 2008:3658-62

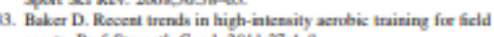

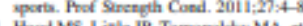

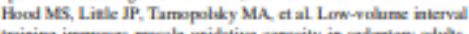

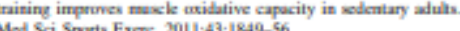

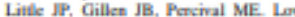

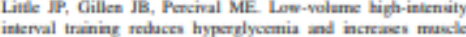

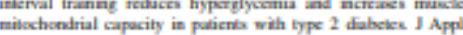
Firyid. 2011:111: 1554-60.

6. Cuken 1. Statistical porwer analypis for the beturiaral weiences. New Yark: Rouliad ge Acadonn; 1908 .

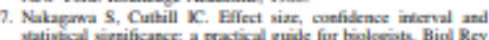

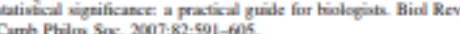

Q Springer 


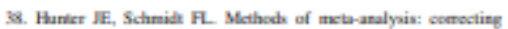

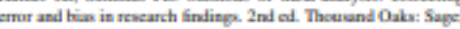

9. Hegim IPT, Sienne IAC, Samovie I, at al. A meriend bool for Mocsing raik of his in randorined trials ls Chandler Costrane Datahues Syd Rev. 2016;1015uppl 1):CD201601.

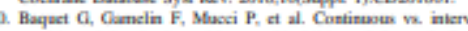

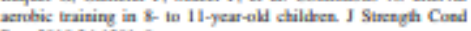
hes. $2010224: 1381-5$

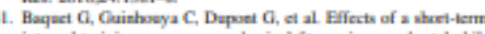

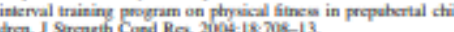

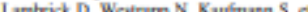

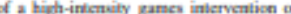
health in young chiliten. I Sports Sci. 2016034:190-8.

43. Noursy C, Deruelle F, Guinhruya C, et al. High-internity intermittent running training inpowo pelmenary fuaction and alkit.

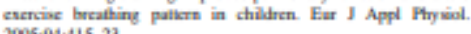

4. Bodty LM, Sration Q. Hacket AF, et al. The effloctinenes of a

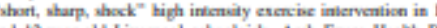
2010:44:19-25.

5. Spetich B, De Martea M. Kostilet K, et al. Effects of 5 wecto de

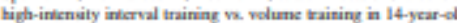
woser player. I Sinerith Coed Re. 2011:25-1271-

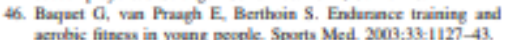
actubic fitses in yoeng poople Sports Med 2003; 33-1127-43.

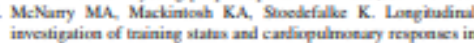

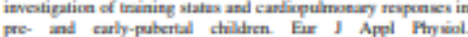
pre- and carly.p.p.

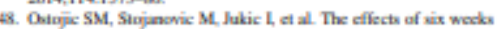

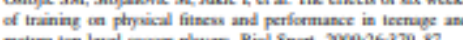

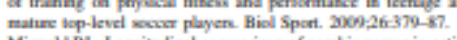

9. Mirwald RL. Leagitudinal corrpariocn of actubic power in active and inactive bs

9. MeNary MA. Welman JR, Jeno AM. The influsace of tricinizg

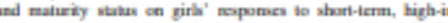
lesaìy upper- and lowat
51. Funtilues AN, Noramo E, Bawaked RA et al. Impast of diet on canfometabolic heath in ctlden and adolocent. Nutr 1 (14:118

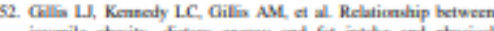

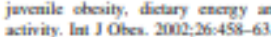

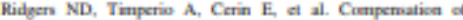
propesal activity and sedentary time in primsy schod chilater. Med Sci Sports Frem. 2014:46:1564-9.2

54. Ruwlend TW. The bidogical hasis of phyaical activity. Med Sei

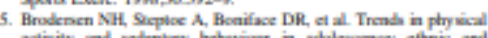

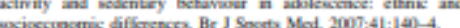

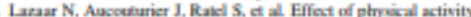

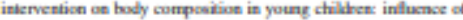
bady mas index states and gender. Acta Pacdiat. 2007:96:1315-20.

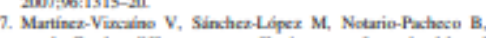

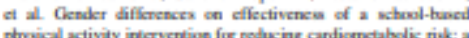

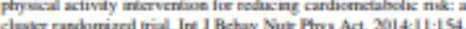

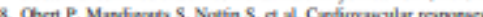
to enderace trairing is tiliter eflod of pendr. Ear $1 \mathrm{Cin}$ Imeatif 2003;33:190-208.

9. Chrietion D, Todd C, Hell R. at all Active children thrsupt incrtive rouchers-evaluation (ACTVE): a mised-mecthod feaAnility tudy. BMC Public Heath 2016:16:800.

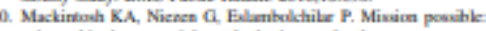

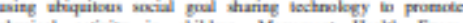

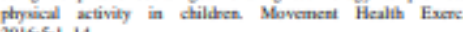

61. Fus KR, Cosper A, MeKenna I. The sethorel and prometive of

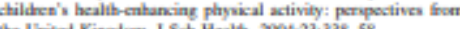
Couriga sa. Fith $\mathrm{s}$.

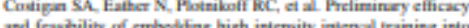
the school dry: a pilut randominod cuatoded trial. Prer Med Rep. 2015:2073-

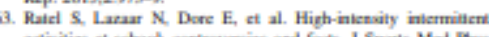

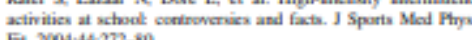


Appendix B - Eddolls, W. T. B., McNarry, M. A., Lester, L., Winn, C. O. N., Stratton, G., \& Mackintosh, K. A. (2018). The association between physical activity, fitness and body mass index on mental well-being and quality of life in adolescents. Quality of Life Research.

Qually of Lin hesanch

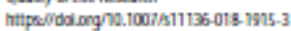

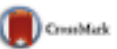

The association between physical activity, fitness and body mass index on mental well-being and quality of life in adolescents

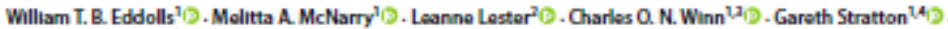
Kolly A. Mackintosh ${ }^{10}$

Acroptod:8 hine 2018

eThe Nuthorpit 2018

Abstract

Purpose The purpose of the current study was to imvesligate the mediabory role trtween viporuus physical activity, bod muss inder (BMD), and cardionespinitury fitness on symptoms of depression and their suboequent dirct and indinect effects on quality of lie (QoL).

Mathods Five hundred and swerty-six adolescents' (314 boys, $12.5 \pm 1.1$ yeass) physical artivity kevelk, cardicaspinialory fitness, BMI, levels of depressive symptoms, and Qol. were measured. Structural equation modelling was used to evaluate the differenx in linear structural aswociations between variabies.

Rasults The modil suger sted that cardiorespinatury fitness $(\hat{\phi}=0.16, p<0.001)$ and symploms of depression $(\beta--0.52$,

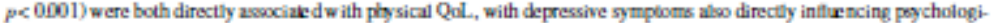

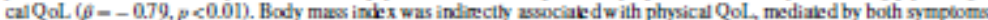
of depres thy by cantionespiration tion

al wity, carlinespinatory timess and dimetly and indirectly, with mental well being and Qd. It could, tix $x$ fure, be postulated that enhancing cardiom:pinintory fitmss and BMI thruugh increasing vigorous pbysical activity may beneficial to both mental well-being and Qol. in adolescents.

Kaywords Physical activity - Candionespiralury fitness - Body mass index · Mental well-being - Quality of life Adolescerts

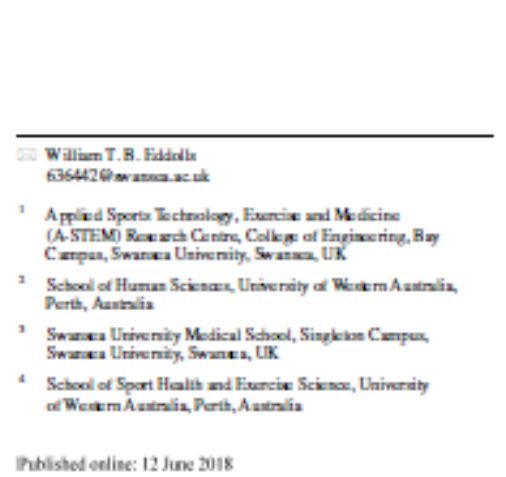

Introduction

Quality of bie (QoL), a sutsect of iralth defined by the Wurld Health Orpanisation to include the plysical, mental and social well-being of a person [1], is widely recognixed as 2 fundumental element in the evaluation of populasion health [2] and should be consider the underlying target for health interventions [3]. Nonetheless, despile its widely accepted

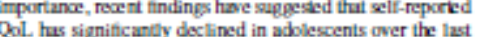
iccade [4], highlighting the need fir intevertions that me. ciflcally tanget Ool in this population. Howe ver, due is the Thective naure Q Qal, it cannot be direets enhanced (S). nets further ifiention as potential mediasos through which benhance Qol. 
Pavious nesearch has shown that intervensons thas mod fy certain health ar lated parameles associaled with Qol. vach as physical lealth and merial well-being, can indirectly

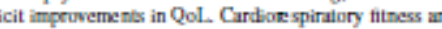
ody mass inder (BMD), inter-related indicators of healt 6). paedict QoL_; those with a higher candioaspindory fit bess ex perie noe a beter QoL. [7], and owenweight or obe individuals typically exrr riencing a poorer $\mathrm{QoL} / 81$. Curs crespiratury fthess and BMI hne also ben associaled with

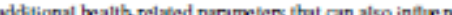

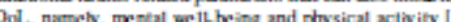
Q1., nam 0. Inied, arearch has sugessided that werwe ight or obese

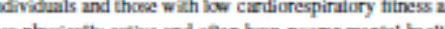
ess physically active and conen hene pooner mental tratt. Specifically relating to QoL., those suffering from ment: $r$ at th isers, or not moeting physical attivity guidelines of $\omega$ min of moderale-bo-vigurous paysical activity per dis. ire at greater risk of a pour QooL [11, 12] However, despis hese predictors being identified, the interaction $x$ twoes them and the influence of mertal health on QoL remzin olential indimet milationohips be health rhaviours ind the ir modieting and interactive influence on QoL. Fir ex ample, when compared to moderate physical activity. vigoeuus physical activity has been associated with fewe symptoms of dipression [13], the moet common form menial illness in the UK [14], and has also been shown medict cardionespindory fitmess and adiposity [10]. Thus, vigorous physical activity levels have the polential to indrectly affect Qol. through sympioms of depression, adipcris and cardionasculir fithess,

Therefore, the purpose of the curnent study was to imves Egeve the militionships between nigorous physical axtivity, BMI and cardions spiralory fitness on sympioms of depres son, and their subsequent dinet and indirect effects on $\mathrm{Ool}$ using structural equation model lling.

\section{Methods}

\section{Participants}

Five hundred and wenenty-six adolescents ( 314 boys $12.5 \pm 1.1$ years) book purt in the stucty. Following parental consent and child assent, measures were taken over appros imately 6 weeks at the beginning of the academ

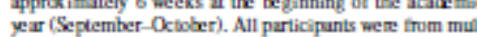
Eple, randomly stratitied, comprehensive schools located Eple, randomily siratified, comprebensive schools located in South Walex, UK. Approwil to conduct this research wis granted by the insolusional ethical abvisory committex (ethics number. PG/2014/29) and pericemed in acocentence with the Declaration of Helsinki.

\section{Measures}

Anthropometric and maturity assessment

Stature and body mass were measured, to the $\mathrm{x}$ arest $0.1 \mathrm{c}$ ind $0.1 \mathrm{~kg}$, respectively, uxing a stadiomeler (Seca 213, Sece Lid. Birmingtam, UK) and body mass scales (Seca 809 Seca Lisd, Birmingham, UK). From the measures, BMI

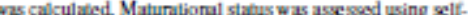
reporied indices of pubic har, as described by Tanner [15]

\section{Physical activity levels}

The GT3X + (ActiGinaph, Pensacola, FL, USA) was used so measure and recoed the quantity and frecir noy of body movement, providing a valid and reliable objective measure of physical activity [16]. Participonts were asked to wear the acceleromeler, st at $100 \mathrm{~Hz}$, on the ir right mid zxilla the acceleromeist, st af $100 \mathrm{~Hz}$, on tre ir nigh mid- zxilla line at the level of the iliac crest fur seven full days, only removing it if they unde riook contact or waler-based activises. Wear-time dirries wer wed to log why tre accelerumeles were taken off and for how long. Data were analysed using KineSoft (uersion 3.3.67; KineSoft, Suskatche wan, Canada) employing $1 \mathrm{~s}$ epochs with sutained r riods of at least 20 min is mero counts considered nog we ar time [17]. A minimum drily wear time of $10 \mathrm{~h}$ foe 3 deve, including

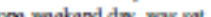

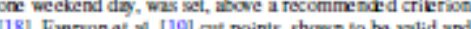
be valid and mants of attivity iniensity in childen and adolescents [20], wene used to calculate vigurous physical ativity 6.4012 counts per minule).

\section{Depressive symptoms messurament}

Symptoms of depression were measured using the Cenier for Epidemiologic Studies Depression Scale fie Children (CES-DC) qx sliunnaire [21], which has been vilidated for use within the adolescent population [22]. The CFS.DC is 120-item scule with overall scores ranging from 0 is 60 with higher scores representing a grealer ievel of depreswive sympinms. Inkernal melizhility fisr thes scale, based on Cronbach's Alpha [23], was dermed good ( $x=0.91$ ).

\section{Quality of Iffe messurement}

Perceived Qoll. was assessed wing the Pediatric Quality of Life Imvenbory (PeddOL) Teenaper Report, Version 4.0 [24], a widily validated 23 -ik m scale designed for uee with perEcipants aged between 13 and 18 years $[25)$. Within the construct of the 23-item questionnaire, the me zure is broken down into four scales: physical ( $n-8)$, emotional $(n-5)$, 
social $(n-5)$ and school functioning $(n-5)$. Physical $(n-8)$ and prychological (n- 15) Ool, summery scones were calcy. live. Soores nanged between 0 and $M 0$, with higher soom: indicasve of a better Qol. Iniemal meliahilaies for physica Qol. $(\alpha-0.759)$ and paycholggical Qol. $(\alpha-0.875)$ were demed acceptable [23].

Cardlorespirstory fitness

Candiorepintory fitrxs was eximuled using the multi-stap fitness bist, a previousty valdated field meroure in chillte [26]. The multi-stax fithess best was conducied over a 20-m [26]. Trex, in a hart-ifloor indoer sports hall, with the total num-

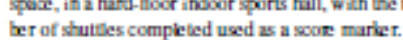

\section{Statistical analysis}

Analysis was guided by Ferrarset al. [2T] conceptual modk which hypothesised that measured fralth outcomes can pre. dict Qoll. Data were analysed uxing IBM SPSS Statides for Windows, Version 220 (IB M Corp, Armonk, NY, 2013) and IBM SpSS AMOS for Windows. Version 220 (IBM Corp, Armonk, NY, 2013). Missing dita ( $n=903,17.4 \%$ ) were imputed using anexpectalion-maximisabion al gunithn. Structural eçuasion modelling was ued to evaluate the dis ference in linear structural associations betwoen variable: Direct effects were estimated using dinect path ooefficients betwern two me zured variables and indinect associations werr estimated as a product of two dirct effects betwer three measured variahies. Given the polential confounding

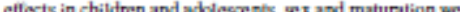
rcoound for win the model. All dita ane proventes

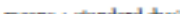

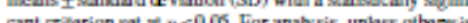
stated dowere standardised ( $p$ ).

\section{Results}

The means and SD of the me arued variabes of the chlilden and adolesxnts that purticipated in this study, dichotomise by ser, are presertied in Tahk 1 .

\section{Confirmatory factor analysis}

Inspection of the moxil ft indixs (NFF - 0.981, TH - 0.982 CF -0.991 , RMSEA <0.06) saspested that the modil was acoptable. Stancturdived purameler eslimates for the mex: uneme nt: model are prowided in Fig, L.

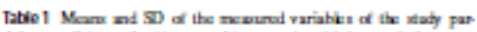

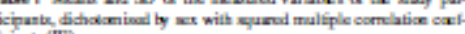

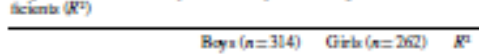

\begin{tabular}{llll}
\hline BMII & $2.93 \pm 4.11$ & $20.69 \pm 3.74$ & 1.04
\end{tabular}

MSFT $\quad 469=23.1 \quad 33.2 \pm 147 \quad 360$

GSDC $\quad 143 \pm 10.0 \quad 30.3 \pm 11.6 \quad$ Ds

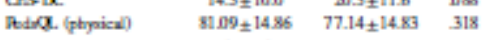
$\begin{array}{llll}\text { Radiad (poychological) } & 78.58 \pm 15.79 & 73.30 \pm 16.97 & .620\end{array}$ \begin{tabular}{llll}
$\mathrm{va}$ & $2609 \pm 10.21$ & $22.75 \pm 7.64$ & 032 \\
\hline
\end{tabular}

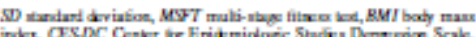

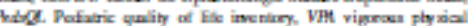
stivity

\section{Structural equation model}

Standardised and unstandardised regression weights with standard errors between variables included within the model are prowided within Table 2 .

\section{Vigorous physical activity}

The model (Fig. 1) revealed that vigarous physical attiv. ty was directly related to BMI $(\beta--0.29, p<0.001)$ and cardiurespiratory titness $(\hat{\rho}-0.24, p<0.05)$, and ind rectly related to depressive symptoms scores $(\hat{\phi}=-0.03$, $p<0.01)$ and physical Qol $(\beta-0.04, p<0.001)$, med aled by BMI and candiomespiratory fitness, respectively.

\section{Body mass index}

Body mass index was directly related to cardicmespiration fitness $(\hat{\beta}=-0.33, p<0.901)$ and deparsive symptoms sours $(\beta=0.12, p<0.05)$. Indirect relationships were found between BMI and physical QoLL, medialed by cardiomespiratory fitness $(\beta-0.05, \beta<0.001)$ and symptums of deparssion $(\hat{\phi}=-0.06, p<0.001)$. Additionally, an indirect relasionship was found between BMI and poychological Qol. $(\beta--0.09, p<0.001)$, mediated by symptoms of dipression.

\section{Cardlorespiratory fitness}

Candiomspinalory fitmess had direct and indirect effects on QoL me asures, cardionespiratory fitness socess had a dinct relationship with physical $Q \circ L(\beta-0.16, p<0.01)$, and an

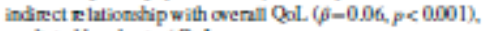
mediated by physical $\mathrm{Qol}$

Q Sprioger 


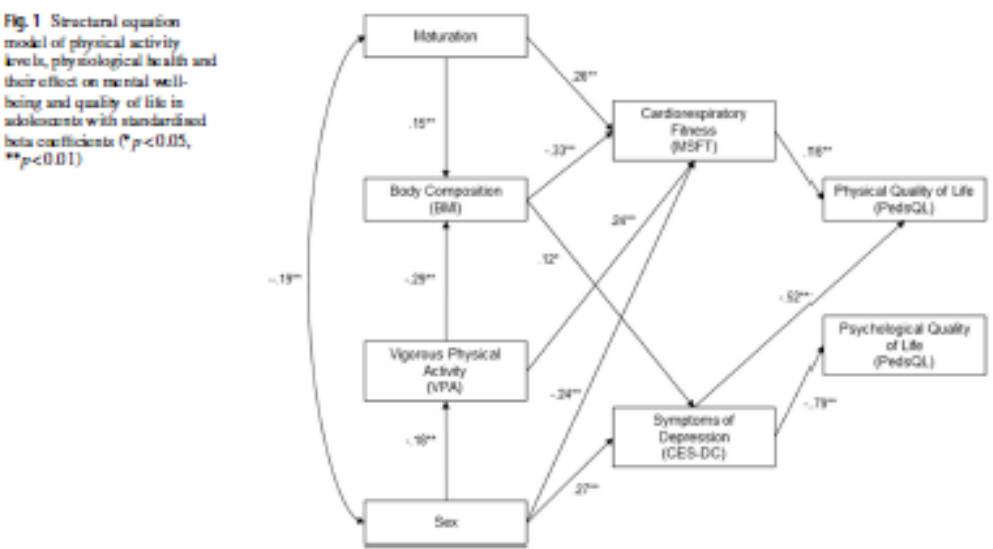

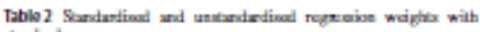

\begin{tabular}{|c|c|c|c|}
\hline Path & $\beta$ & B & Sㅍ \\
\hline $5=x \rightarrow \operatorname{VaA} *$ & -0.18 & -3.34 & 0.76 \\
\hline Mataration $\rightarrow$ BMe* & 0.15 & 0.55 & 0.15 \\
\hline $\mathrm{WA} \rightarrow \mathrm{BM} \cdot \cdot$ & -0.20 & -0.13 & $\mathrm{om}$ \\
\hline Mataration $\rightarrow$ MSTT"* & 0.26 & 0.53 & ong \\
\hline $\mathrm{BMI} \rightarrow \mathrm{MSF}+*$ & -0.33 & -0.18 & $\mathrm{om}$ \\
\hline $\mathrm{BM} \rightarrow \operatorname{CES}-\mathrm{DC}^{\circ}$ & 0.12 & 0.33 & 0.11 \\
\hline $\mathrm{VA} \rightarrow \mathrm{Mg} \mathrm{T}^{*}$ & 0.24 & 0.06 & $0 \mathrm{Dt}$ \\
\hline $\operatorname{sex} \rightarrow \operatorname{css} D C^{*}$ & 0.27 & 604 & 0.59 \\
\hline $\mathrm{Sex} \rightarrow \mathrm{MST} *$ & -0.24 & -1.04 & 0.15 \\
\hline CS:DC $\rightarrow$ Podid . (pophological)" & -0.79 & -1.17 & 0.04 \\
\hline MSFT $\rightarrow$ Padra. (phyniay)" & 0.16 & 0.95 & $0 . x$ \\
\hline CrS DC $\rightarrow$ Podia (phy ried)" & -0.52 & $-0 . x$ & ons \\
\hline
\end{tabular}

QoL $(\beta=-0.20, p<0.001)$ and psychological Qol $(\beta=-0.61, p<0.001)$

\section{Maturation and sex}

Maturation directly affected BMI $(\hat{\beta}=0.15, p<0.001)$ and cardiar ppinalory tithess scores $(\beta-0.26, p<0.001)$, whilst set was found to directly affect vigurous pbysical activity $(\hat{\theta}=-0.18, \rho<0.01)$, cardiorespinticy fitmess $(\beta-0.24$, $p<0.001)$ and deparsine symptoms $(\beta-0.27, p<0.901)$.

\section{Discussion}

This study investigated the relationship and mediatory effects bebreen physical activity levels, BMI, candicasp. natory fitness and sympeoms of depression, and the ir sub. sequent effect on physical and poychological Qd. Models sappested that viporous physical activity, cardicaspintory theess and BMI were associated, both directly and indirectly. with mental we li being and physical and poychological onl

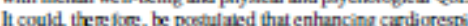
cle line atory tiness and B wat trough increasing vigoosus physical attivity may be be neficial to both mental well being and
Qol in abolescents.

Depression

There was a direct relationship between symptoms of sepression and peychological $\mathrm{Q}$ oL. $(\beta=-0.79, p<0.01)$ and physical $\mathrm{QoL}(\beta--0.52, p<0.001)$ and an ind:met relationship with overall $\mathrm{QoL}$ mediated by physical

The current mesults show that, in accond with previous linerature [28], a healthier BMI is associated with iver symptoms of depression. It is possibit that this association. at kat in purt, may $\mathbf{x}$ due to poychosocial facturs associ. aled with BMI. For example, stigmatisation towards those

S) Sprisgr 
hat hue a higher BMI may cause lower selfe stoem, induse segative self-imaxs and lead to perceptons of social is Iation, thus eliciting de pressive symptoms [29]. A revice by Markowize et al. [30] also proposes that the associsson between obx sity and dipression is related to conxr rganding sif-perceived health and appearance, wherehy those with higher BMI experience, or ine mon vulnerable to, deparsion

As alluded to previousty, symptoms of deparsion were 2isocialed with physical and psycholoy ast Inderd, coneruent with previous reseirch [11], findings from the present study suggest that lower levels of deparssive symptums were aswocianed with higher levels of poychological and physical Qol. highlighting the link between me rital well-being and Qoll. In addition to the dinect associations with depressive sympinms, the model also proposes that a ralthier BMI is indinectly associated with a grealer poychoopgical and pbysical QoL, medialed by levels of depresswe symploms and cardionspiralogy fitnex, mespectively. As out. lined in previous literature [31], the findings, therefore, identify BMI as a bey influential facbor that can significantly

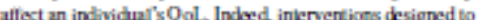
mporve Q

Beyund depressive symptoms and BMI, participants with a higher candiomspintony titness also experienced a better plysical Qd. [7]. Given the link between cardiorespiralory fitness and BMI obsenved in this study and elsewhem [33]. the associalion between cardiorespiralury fitness and physical QoL [34] may also be miated to a negative self-image and selfesjeem [29], components that are encompersed within the Qol. measurement. In addition to prychosocial

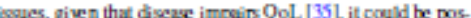

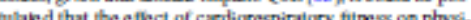

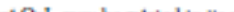
in mechaniturs explaining the connection between cardions. piratory fitness and Qol. ane not entimely understood [37] and, therefure, wurnat further meganh. Subsequently, future interventions sseking to enhanx Q ol, should remain mulsfaceled, simultarousty improwing candorespiralory fitrsa, BMI and sympioms of depression.

A furtier inieresting finding was that, whilst higher levels of vigurous physizal activity weme directly associaled with lower BMI and increased candiorespiratury fitness [38, 39]. hipher kevels of vigceous physical astivity were also ind. rectly associated with fewer sympocms of depression, mediand by BMI. It is postuland that, the negative correlation retween vigorous physical activity and BMI may be associned with impruvements in sy mploms of depression, potentially due to greater megulation of the hypochalamic-pitu. tary-airenal zxis and insulin control, we well as improwed self-perceplions in both appe inane and he alth $(20,30,40)$
Consequencly, increasing vigoeuus physical activity may elicit improvements to mot conly a person's physical tralth but also mas improve mentai well beirq. Indoed, For [4] found that ir althy adults should consider vigorous e se rise is a mears of impruving mentall well-being.-However, with the exaption of Partitt et al. [42] and Gerber et al. [13]. who found vigorous physical activity elicited mental health benefits beyond those engendered trough bow and moder

ale physical activity, there is limited regearch that focuses on the effects of viporcus physical artivity on mental we ring, expecially in chiltren and adelescents. Ginen these

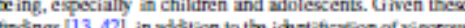
the physical actovity as 2 moderatur or mentar beal th within the

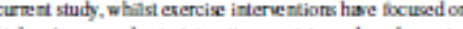
high volume, modenuk-intensity ar resistance-bured exercis [43], allernative funms or intersities of evertive may be mon: beneficial. Subsequently, dspile previous mied findings [44, 45], the curnent study presents a newiound advocasy for the potential of high-intensity exercise, a siructured form of vigurous pbysical activity, to directly and indrectly improwe symptoms of depression and physical and psychological

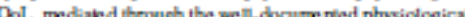
ralth ber fits [46].

Congrin it with pevious reseanh [47], the present study in monstrated that sex had a significant relationship with both cardicrespiratory fitness and symptoms of depression: girls were more tikely to ex perience poorer finess and mental well-being. These differences may, in purt, be related bes irummental corsidentions, fir ex ample, sociocultural roiks and poychological attribules [48] or hormonal facion: (49). However, it is also important to consider the importance of the diverpenx in physical development during

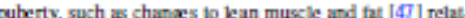

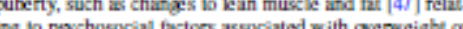
(n) obesily $[20,30]$, therefure, further highlighting the need for $\mathbf{r}$ ath interventions to primarity focus on imperving BMI benhance werall tralth

Thes is the tirst study to use structural equation modelling to delermix boch direct and indinect associations between dhysiological health, physical axtivity levels, mental well. ring and Qol. Furthermore, the present study accounied for maturational states and sex, boch of which are often overlooked in child and adolescent studies, w well as uxing robust, previously validied, meavuns of plysical attiv.

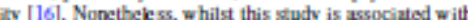
rumerous strengthe, there are inevitably some limilations. Wis a within the model, given its well-established melationship with the measured variables $[90)$, may limit the interpretaEon of the findings. Despile its frequent utilisalion within clinical mesearth, the application of BMI is commonly sabject to scepticism due to the measure's inabiliny to dif. ferentiale between kan and tat body mass [51]. Additiona 
Imitations inchude the self-reporting of mertal well-being und tix associated disabtuntages, such as social desinability [52]. Furthermore, for the QoL, measurement a number of participants fell below the minimum age threshobd fur the Teenager Report (13-18 tears), however, given that them are only small differences bebreen Child Report and Teenage Report (i.e., mpplacing the woed child with kenaner), although it cannot be ruled out, it was demed unlikely to have influenced the results. However, all of the questionsinted acceptable reliability. Finally, primarily dx is perexpectation-max imisation was utilised to impute missing dath, and whilst it has previously been reporied as a valid method [53], it is important to acknowede the influenx the absencr of a complete dare set mas hwe had on the findings within this stuady.

\section{Conclusions}

In conclusion, the present study found that vigurous physi-

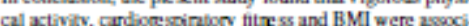
sied, both dinetly and indirectly, with mental well being and physical and poychological Qol.. As such, it could be and physical and poychological Qol. As such, it could be by increasing vigorous physical activity may be beneficial to boch mental well being and Qol in adolescents. Subser. cuently, our findings supgest that, to elicit improve ments to Qol, exercise or physical axtivity interventions should remain multi-faceted, simultaneously focusing on candiores pintory fitrss, BMI and symptoms of depression.

Acknowlodgements The authors would like to thenk the atat and

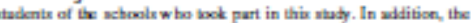

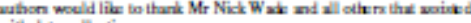
witt data crilection.

Funding William Fidolle, Melitta MeNery and Kelly Madintruch

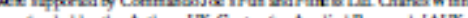

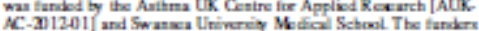

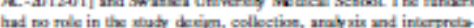

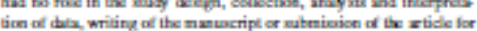
putlication

Compllance with ethical standards

Conflict of imbarest Wilium Fiddile Mdits MeNary, Clarle Wirn,

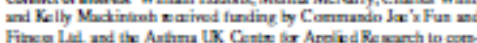

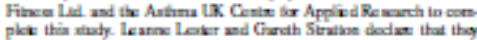

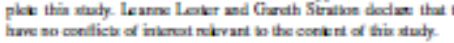

Ethical approval Approwal in condact this newath wax granied by

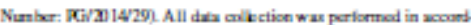

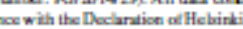

Intormed consent Written parental on pardian concent and child

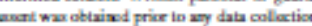

Open Access This articke in dietribubd under the terma of the Cres.

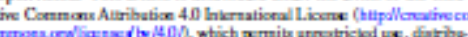

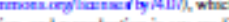

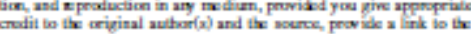

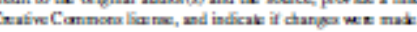

\section{References}

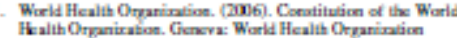

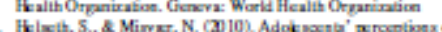

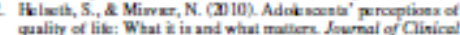
Quality of lik: What 2 in and wit.

3. Ramen-Sictere, U., Heriman, M., Derix, J., Oten, C., Ball$\checkmark \mathrm{C}$.

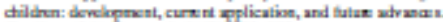

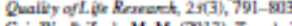

Cai, W., 2 Zad, M. M. (2013). Tands in balth-rilued qual.

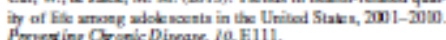

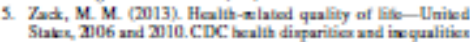

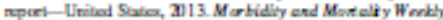
Repori, 62, 105-111

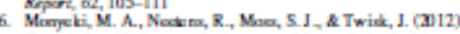
The rdaticentip batween body armpenition and phy rical tix: in 14 yor old adolocents sniding within the Takwe loo Feath $12,374$.

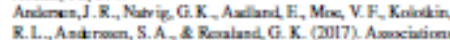

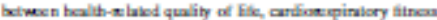

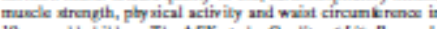

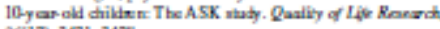
20(12), 3421-34x

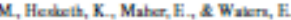

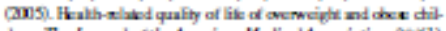

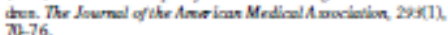

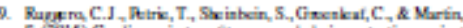

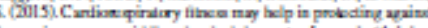

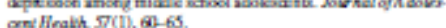
Purik, T, s sintion $G$.

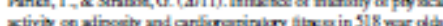

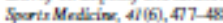

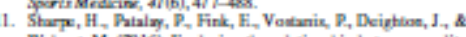

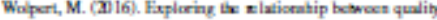

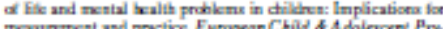

Dining $25(6) 650-60$

M. R. Ahrad, A., Zaination, 8. Imail, K.F.Aang, M, M. T, \& Ywod, N. A. (a) 16). A vorix

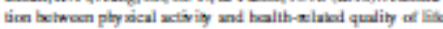

을 spinger 


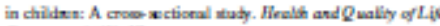
Ontovers, $14(1), 17$

13. Griser, M, Brand, 5 , He

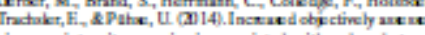

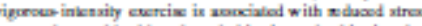

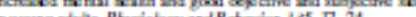

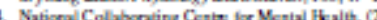

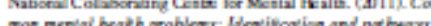

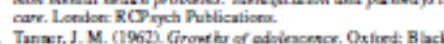

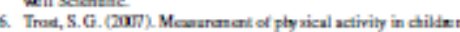

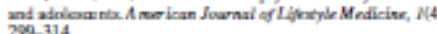

Cuklier, D. J., H zernan, D. J., Merrny, D. M. Addy, C. L..,Con

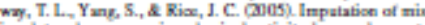

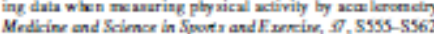
8. Rich, C, Gersei, M., Gritthn, L., Sera, F, Derakex, C. Cortina-Boriz, M. (A) 13) Quality craturd methodr in acak

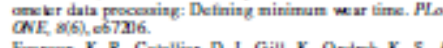

9. Finemon, K. R., Craellier, D. J., Gill, K., Ondrik, K. S., MeMurny, R.G. (anos) Calitration of two objuctive manus of phy nical activity

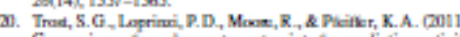

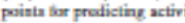

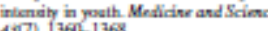
Wisman. M. M.

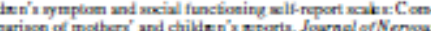

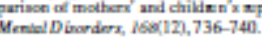

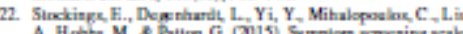

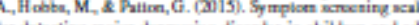

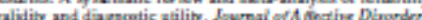

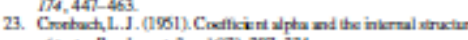

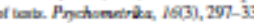

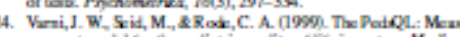

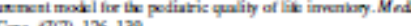

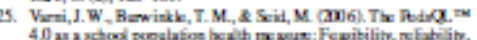

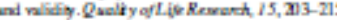

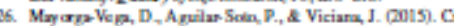

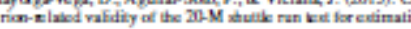

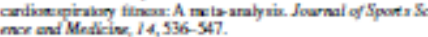

1. Fine C.E. 7 .

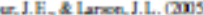
Conoptaal modil of bolth-slaked quality of lik. Jowrnal of

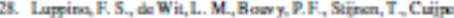

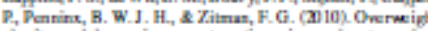

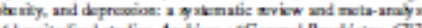

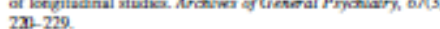
2al-220

.

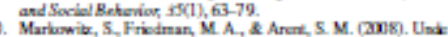

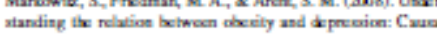

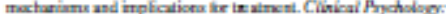
Schimse and Pration, 15(1, 1-X).

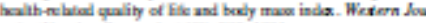

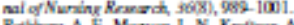

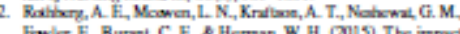

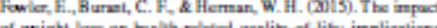
of weight has on wath-ritided quality of-lik: implications $1371-13 / 6$.

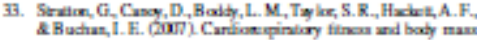

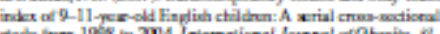
$1172-178$.

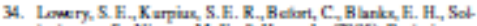

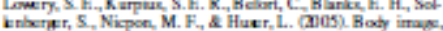

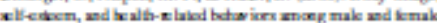

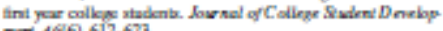

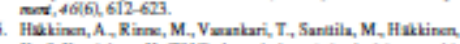

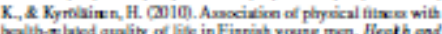

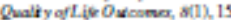

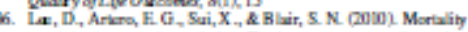

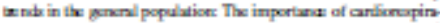

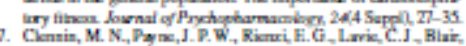

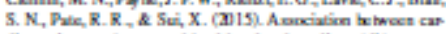

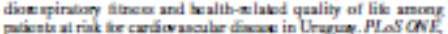

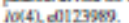

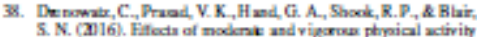

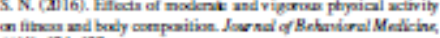

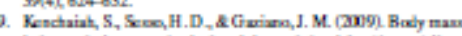

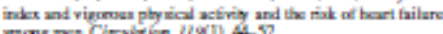

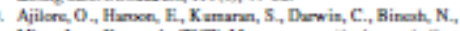

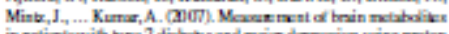

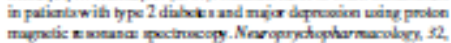

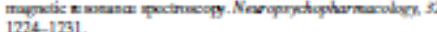

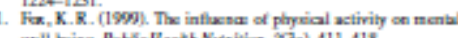

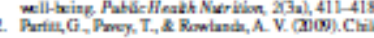

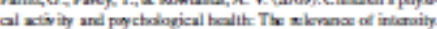

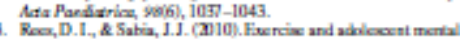

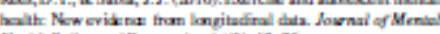
Reath Poligy and Econsmiex 19(1), 13.

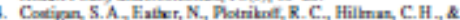

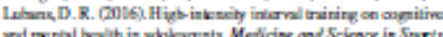
and Finein, 45(101, 1985-1993.

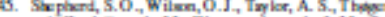

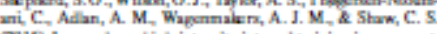

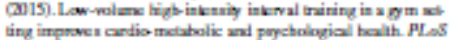

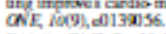

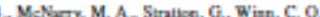

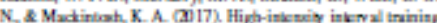

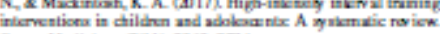
Sport Medinin, $f(11), 2363-2374$. 


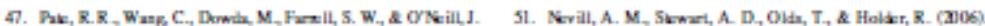

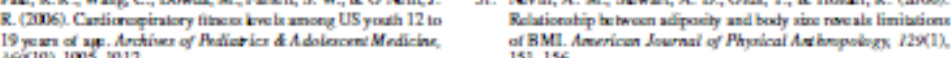

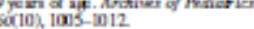

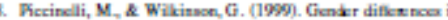

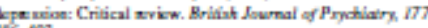

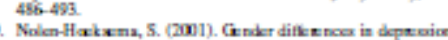

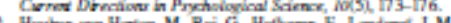

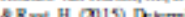

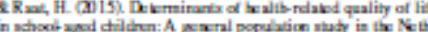
151-156

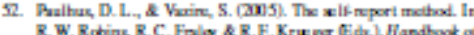
R. W. Roding, R.C. Faly B. R.F. Krugar Gide), Handbook of

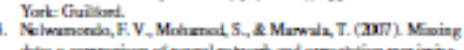

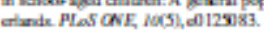

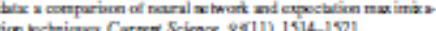




\title{
Appendix C - Headteacher information sheet (intervention)
}

\author{
Applied Sports Technology Exercise and Medicine Research Centre (A-STEM)
}

Sport and Health Portfolio, College of Engineering

\section{HEADTEACHER INFORMATION SHEET (Intervention)}

(Version 1.2, Date: 01/06/2015)

Project Title:

The x4a trial: eXercise for Asthma with Commando Joes.

Contact Details:

William Eddolls

Charles Winn

Dr Kelly Mackintosh

Dr Melitta McNarry

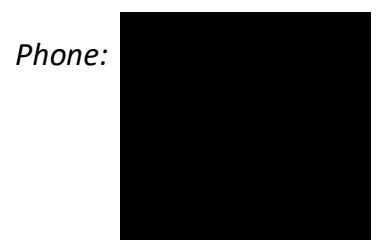

Email:

1. Invitation Paragraph

Thank you for taking the time to read this information sheet, it will provide you with the details of our study and hopefully provide you with the information you require to help you decide if you want your school to participate. It is important to say at this point that the decision to take part is entirely up to you and that your school will not be at a disadvantage for future studies should you decide not to participate.

2. What is the purpose of the study?

A strong relationship has been shown between physical inactivity and obesity. Worryingly only a small portion of children meet the government guidelines of 60 minutes of exercise. This, in a lot of cases, is because time constraints do not allow children to get the exercise they need. Short bouts of high intensity exercise have previously been shown to be an effective and useful way of meeting exercise requirements. Therefore, the purpose of this study is to assess whether high intensity training can be used as an effective method of improving numerous physical and mental health factors including asthma symptoms and quality of life.

3. Why has your school been chosen?

Your school has been selected via random assignment after being selected as a potentially suitable school. The results from the initial questionnaire will help us identify pupils with asthma and we would therefore like to involve them within the study.

However this does not mean your schools has to take part in the study. This is voluntary and you have the right to withdraw your school from the study at any time. If you wish to withdraw from the study you will not be hassled, get in trouble, and you will not have to give a reason for your withdrawal. All participants will be able to withdraw themselves from the study at any point without fear of penalty on withdrawal. 


\section{What will happen to your pupils if they take part?}

Once consent/assent is returned students will be invited to attend our exercise programme. This is a 45 minute exercise session that includes 30 minutes of high intensity exercise, conducted 3 times a week before or after school. These sessions will alter weekly and last for 6 months. Over the course of the intervention this totals approximately 54 hours. An example session would be planned as follows:

Warm-up - This will last approximately 5 minutes. In the warm-up the students will take part in light jogging and stretches to ensure their body is ready to exercise.

High intensity exercise - This will consist of numerous bouts of 30 second exercises followed by 30 seconds rest. Exercises will include numerous military style games. This section of the programme will last 30 minutes.

Warm-down - This will be conducted to ensure that the children experience minimal muscle soreness following exercise. This will last 10 minutes to allow for possible asthma related issues.

All pupils will also be invited to an assessment day where a number of tests will be conducted. These assessment days will occur at 5 time points across approximately 12 months but should only require the students to miss half a day of lessons per time point. This will total approximately 25 hours. These tests will include:

Questionnaires - These will include quality of life, mental well-being, asthma health utilization, asthma control and an anxiety questionnaire.

Lung function test and fractional exhaled nitric oxide - Participants will be asked to blow hard into one device and gently into a tube connected to a monitor, these will be taken 5 times throughout the year.

Maturation assessments - The children will be asked to complete a questionnaire. It consists of showing line drawings of different pubertal stages. A box next to each picture is then ticked by the child to identify which pubertal stage resembles them. This will be done in complete privacy and only looked at by researchers on completion of the study. Please see attached sheet for line drawings.

Multi-stage fitness test - The children will be asked to complete a bleep test to assess their fitness at different points throughout the year. It will require the children to run $20 \mathrm{~m}$ shuttle runs to the sound of beeps which increase in speed until the child no longer reaches the line before the beep sounds.

Focus groups - In this the children will be interview in groups of 4-6 and asked questions related to exercise and asthma. These sessions will be either videoed or voice recorded. Those that are randomly selected to attend will be asked to provide us with 1 hour of their time.

Physical activity measurement - Children will be asked to wear a physical activity monitor for 7 consecutive days. They will also be asked to keep an activity log to record when they remove the accelerometer. Handing out and collecting the accelerometers will take approximately 1 hour at 5 time points over 12 months, totaling 5 hours. 
A smaller group of children will also be selected for the laboratory testing (roughly 16 per school [8 asthmatic and 8 non-asthmatic]). They will be asked to attend a laboratory session over 4 days (this will be a maximum of 40 mins each session) at 5 time points. These also can be run in your school. During this laboratory testing students will be asked to complete tests that will include:

Functional fitness test - In this test they will practice using the static bicycle until they are happy with it. It will start off easy and get harder until your child says they can't keep going. During this, they will breath into a mask to allow us to measure the air that they breathe in and out. This mask does not make breathing any harder and you can talk through it and remove it at any time they feel uncomfortable about wearing it. The exercise will last approximately 15 minutes.

Blood analysis - A small pin prick will be administered to the children's finger where an extremely small drop of blood will be taken, if the children do not feel comfortable doing this test then they will be able to decline without any question.

Blood pressure and heart rate - Participants will be asked to place an inflatable cuff around their arm. This will inflate to momentarily slow the blood flow to the arm to measure blood pressure. Once the measurement is collected the cuff will be removed. To measure heart rate, a monitor will be worn around the student's chest. This is an elastic belt that fits all. An area will be designated to allow the student's to attach this in private.

Pulse Wave Velocity - in order to measure the health of the children's arteries we will measure the speed at which blood pressure waves flow. In order to measure this we will need to apply a cuff around the neck and upper leg of the children. This measurement should only last 5 minutes.

\section{What are the possible disadvantages of taking part?}

There aren't any significant risks or discomforts within the study. If your pupils follow our instructions which will ensure that you are appropriately warm for the activity then the risks will be minimised. There is a small risk of injury from the activity (as in any Physical Education class); however there will be trained first aiders on hand to deal with any injuries which may occur. Asthmatics taking part in the study may succumb to exercise induced asthma after the exercise is complete; this is the main reason for the warm down as if this does occur it will be roughly 7 minutes after the main part of the programme. If this does occur we have personnel trained in asthma care in this situation that would be able to support the child appropriately with inhaler use.

\section{What are the possible benefits of taking part?}

Your student's physical fitness and mental wellbeing including your student's quality of life could improve through completing this program. It will also help provide crucial answers to research questions in the health and exercise area of study namely asthma, childhood obesity and other effects of physical inactivity.

\section{Will my pupils taking part in the study be kept confidential?}

Although your pupils' details will be taken prior to conducting the study, they will remain anonymous and this would be the same should the study be published. Your pupil's personal information will be stored on a protected computer with a password and personal information will 
be destroyed at the study end (with consent forms stored in a locked cabinet in a secure area, to be destroyed within 5 years of study end). Members of the university will be able to access your information under supervision.

8. What if I have any questions?

If you have any problems or need any further information about the study or the testing then please contact any of the research team using the contact details provided. 


\section{Appendix D - Headteacher information sheet (control)}

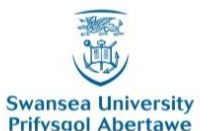

Prifysgol Abertawe

Applied Sports Technology Exercise and Medicine Research Centre (A-STEM)

Sport and Health Portfolio, College of Engineering

\section{HEADTEACHER INFORMATION SHEET (Control) \\ (Version 1.2, Date: 01/06/2015)}

Project Title:

The x4a trial: eXercise for Asthma with Commando Joes.

\section{Contact Details: \\ William Eddolls \\ Charles Winn \\ Dr Kelly Mackintosh \\ Dr Melitta McNarry}

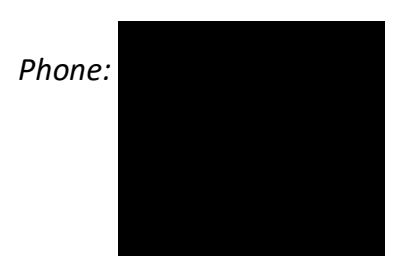

Email:

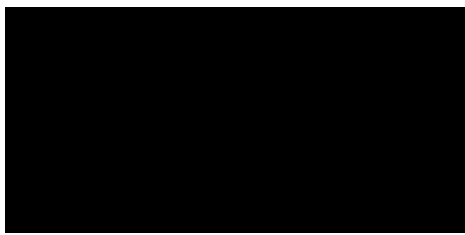

1. Invitation Paragraph

Thank you for taking the time to read this information sheet, it will provide you with the details of our study and hopefully provide you with the information you require to help you decide if you want your school to participate. It is important to say at this point that the decision to take part is entirely up to you and that your school will not be at a disadvantage for future studies should you decide for them not to participate.

2. What is the purpose of the study?

A strong relationship has been shown between physical inactivity and obesity. Worryingly only a small portion of children meet the government guidelines of 60 minutes of exercise. This, in a lot of cases, is because time constraints do not allow children to get the exercise they need. Short bouts of high intensity exercise have previously been shown to be an effective and useful way of meeting exercise requirements. Therefore, the purpose of this study is to assess whether high intensity training can be used as an effective method of improving numerous physical and mental health factors including asthma symptoms and quality of life.

\section{Why has your school been chosen?}

Your school has been selected via random assignment to be a control school. This will mean that similar measures are made on the children as in the intervention schools where the exercise programme is being delivered so that we can tell if the programme has made a difference. However this does not mean your school has to take part in the study. This is voluntary and you have the right to withdraw your school from the study at any time. If you wish to withdraw from the study you will not be contacted further, and you will not have to give a reason for your schools withdrawal. All participants will be able to withdraw themselves from the study at any point without fear of penalty on withdrawal.

4. What will happen to your pupils if they take part?

Once consent/assent is returned your students all pupils will also be invited to an assessment day 
where a number of tests will be conducted. These assessment days will occur at 5 time points across approximately 12 months but should only require the students to miss half a day of lessons per time point (these days will be run at your school). These tests will include:

Questionnaires - These will include quality of life, mental well-being, asthma health utilization, asthma control and an anxiety questionnaire.

Lung function test and fractional exhaled nitric oxide - Participants will be asked to blow hard into one device and gently into a tube connected to a monitor.

Maturation assessments - The children will be asked to complete a questionnaire. It consists of showing line drawings of different pubertal stages. A box next to each picture is then ticked by the child to identify which pubertal stage resembles them. This will be done in complete privacy and only looked at by researchers on completion of the study.

Multi-stage fitness test - The children will be asked to complete a bleep test to assess their fitness at different points throughout the year. It will require the children to run $20 \mathrm{~m}$ shuttle runs to the sound of beeps which increase in speed until the child no longer reaches the line before the beep sounds.

Physical activity measurement - Children will be asked to wear a physical activity monitor for 7 consecutive days. They will also be asked to keep an activity log to record when they remove the accelerometer. Handing out and collecting the accelerometers will take approximately 1 hour at 5 time points over 12 months, totaling 5 hours.

A smaller group of children will also be selected for the laboratory testing (roughly 16 per school [8 asthmatic and 8 non-asthmatic]). They will be asked to attend a laboratory session over 4 days (this will be a maximum of 40 mins each session) at 5 time points. These also can be run in your school. During this laboratory testing students will be asked to complete tests that will include:

Functional fitness test - In this test they will practice using the static bicycle until they are happy with it. It will start off easy and get harder until your child says they can't keep going. During this, they will breath into a mask to allow us to measure the air that they breathe in and out. This mask does not make breathing any harder and you can talk through it and remove it at any time they feel uncomfortable about wearing it. The exercise will last approximately 15 minutes.

Blood analysis - A small pin prick will be administered to the children's finger where an extremely small drop of blood will be taken, if the children do not feel comfortable doing this test then they will be able to decline without any question.

Blood pressure and heart rate - Participants will be asked to place an inflatable cuff around their arm. This will inflate to momentarily slow the blood flow to the arm to measure blood pressure. Once the measurement is collected the cuff will be removed. To measure heart rate, a monitor will be worn around the student's chest. This is an elastic belt that fits all. An area will be designated to allow the student's to attach this in private. 
Pulse Wave Velocity - in order to measure the health of the children's arteries we will measure the speed at which blood pressure waves flow. In order to measure this we will need to apply a cuff around the neck and upper leg of the children. This measurement should only last 5 minutes.

\section{What are the possible disadvantages of taking part?}

There aren't any significant risks or discomforts within the study. If your pupils follow our instructions which will ensure that you are appropriately warm for the activity then the risks will be minimised. There is a small risk of injury from the activity (as in any Physical Education class); however there will be trained first aiders on hand to deal with any injuries which may occur. Asthmatics taking part in the study may succumb to exercise induced asthma after the exercise is complete; this is the main reason for the warm down as if this does occur it will be roughly 7 minutes after the main part of the programme. If this does occur we have personnel trained in asthma care in this situation that would be able to support the child appropriately with inhaler use.

\section{What are the possible benefits of taking part?}

Your student's physical fitness and mental wellbeing including your student's quality of life could improve through completing this program. It will also help provide crucial answers to research questions in the health and exercise area of study namely asthma, childhood obesity and other effects of physical inactivity.

\section{Will my pupils taking part in the study be kept confidential?}

Although your pupils' details will be taken prior to conducting the study, they will remain anonymous and this would be the same should the study be published. Your pupil's personal information will be stored on a protected computer with a password and personal information will be destroyed at the study end (with consent forms stored in a locked cabinet in a secure area, to be destroyed within 5 years of study end). Members of the university will be able to access your information under supervision.

\section{What if I have any questions?}

If you have any problems or need any further information about the study or the testing then please contact any of the research team using the contact details provided. 


\section{Appendix E - Headteacher consent form}

Applied Sports Technology Exercise and Medicine Research Centre (A-STEM)

Sport and Health Portfolio, College of Engineering

HEADTEACHER CONSENT FORM

(Version 1.2, Date: 01/06/2015)

Project Title:

The $x 4 a$ trial: eXercise for Asthma with Commando Joes.

Contact Details:

William Eddolls

Charles Winn

Dr Kelly Mackintosh

Dr Melitta McNarry
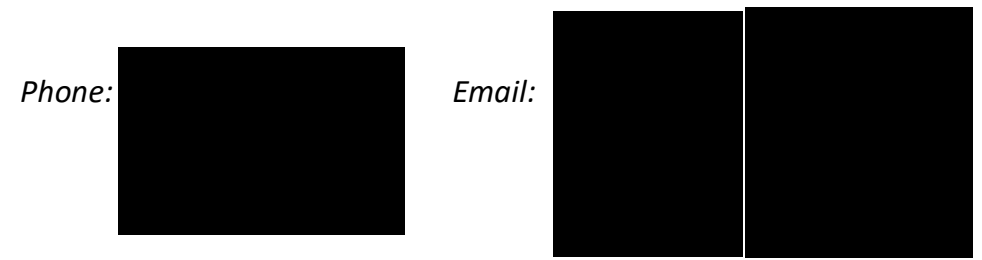

Please initial box

1. I confirm that I have read and understood the information sheet dated 01/06/2015 (version number 1.2) for the above study and have had the opportunity to ask questions.

2. I understand that my school's participation is voluntary and that I am free to withdraw at any time, without giving any reason, without my legal rights being affected.

3. I understand that sections of any of data obtained may be looked at by responsible individuals from the Swansea University or from research regulatory authorities where it is relevant to my taking part in research. I give permission for these individuals to have access to these records. Analysis will be done on anonymous data.

4. I give consent for video and voice recording and photography. (providing parent/guardian have provided consent)

5. I agree for my school to take part in the above study.

Name of School

Name of Headteacher

Researcher
Date

Date
Signature

Signature 


\section{Appendix F - Parental/guardian information sheet (intervention)}

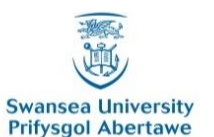

Prifysgol Abertawe

Applied Sports Technology Exercise and Medicine Research Centre (A-STEM)

Sport and Health Portfolio, College of Engineering

\section{PARENTAL/GUARDIAN INFORMATION SHEET (Intervention) \\ (Version 1.2, Date: 01/06/2015)}

Project Title:

The $x 4 a$ trial: eXercise for Asthma with Commando Joes.

Contact Details:

William Eddolls

Charles Winn

Dr Kelly Mackintosh

Dr Melitta McNarry

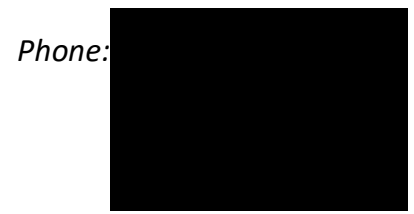

Email:

1. Invitation Paragraph

Thank you for taking the time to read this information sheet, it will provide you with the details of our study and hopefully provide you with the information you require to help you decide if you want your child to participate. It is important to say at this point that the decision to take part is entirely up to you and that your child will not be at a disadvantage for future studies should you decide for them not to participate.

2. What is the purpose of the study?

A link has been shown between exercise and obesity. Worryingly only a small number of children meet the government guidelines of 60 minutes of exercise. This, a lot of the time, is because time does not allow children to get the exercise they need. High intensity exercise has been shown to be a good way of getting enough exercise. So, we want to see whether high intensity training can be used to improve mental and physical health including asthma and quality of life.

\section{Why has your child been chosen?}

Your child's school has been chosen randomly. However this does not mean your child has to take part in the study. This is voluntary and you have the right to withdraw them from the study at any time. If you wish to withdraw your child from the study you will not be hassled, and you will not have to give a reason for their withdrawal. Your child will also be able to withdraw themselves from the study at any point without fear of penalty on withdrawal.

4. What will happen to your child if they take part?

Your child will be invited to attend our exercise program. This is a 45 minute exercise session that includes 30 minutes of high intensity exercise conducted 3 times a week before or after school over 6 months. Over the course of the intervention this totals approximately 54 hours. These sessions will alter weekly. An example session would be planned as follows:

Warm-up - This will last approximately 5 minutes. In the warm-up the children will take part in light 
jogging and stretches to ensure their body is ready to exercise.

High intensity exercise - This will consist of numerous bouts of 30 second exercises followed by 30 seconds rest. Exercises will include numerous military style games. This section of the programme will last 30 minutes.

Warm-down - This will be conducted to ensure that the children experience minimal muscle soreness following exercise. This will last 10 minutes to allow for possible asthma related issues.

Your child will also be invited to an assessment day where a number of tests will be conducted. These assessment days will occur at 5 time points across approximately 12 months but should only require your child to miss half a day of lessons per time point. These tests will include:

Questionnaires - These will include quality of life, mental well-being, asthma health utilization, asthma control and an anxiety questionnaire.

Lung function test and fractional exhaled nitric oxide - Participants will be asked to blow hard into one device and gently into a tube connected to a monitor, these will be taken 5 times throughout the year.

Maturation assessments - The children will be asked to complete a questionnaire. It consists of showing line drawings of different pubertal stages. A box next to each picture is then ticked by the child to identify which pubertal stage resembles them. This will be done in complete privacy and only looked at by researchers on completion of the study. Please see attached sheet for line drawings.

Multi-stage fitness test - Your child will be asked to complete a bleep test to assess their fitness at different points throughout the year. It will require them to run $20 \mathrm{~m}$ shuttle runs to the sound of beeps which increase in speed until they no longer reach the line before the beep sounds.

Focus groups - In this the children will be interview in groups of 4-6 and asked questions related to exercise and asthma. These sessions will be either videoed or voice recorded. Those that are randomly selected to attend will be asked to provide us with 1 hour of their time.

Physical activity measurement - Children will be asked to wear a physical activity monitor for 7 consecutive days. They will also be asked to keep an activity log to record when they remove the accelerometer. Handing out and collecting the accelerometers will take approximately 1 hour at 5 time points over 12 months, totaling 5 hours.

Your child may also be selected for the laboratory testing (roughly 16 per school [8 asthmatic and 8 non-asthmatic]). They will be asked to attend a laboratory session over 4 days (this will be a maximum of 40 mins each session) at 5 time points. These also can be run in your school. During this laboratory testing the children will be asked to complete a number of tests that will include:

Functional fitness test - In this test they will practice using the static bicycle until they are happy with it. It will start off easy and get harder until your child says they can't keep going. During this, they will breath into a mask to allow us to measure the air that they breathe in and out. This mask does not make breathing any harder and you can talk through it and remove it at any time they feel 
uncomfortable about wearing it. The exercise will last approximately 15 minutes.

Blood analysis - A small pin prick will be administered to the children's finger where an extremely small drop of blood will be taken, if your child does not feel comfortable doing this test then they will be able to decline without any question.

Blood pressure and heart rate - The children will be asked to place an inflatable cuff around their arm. This will inflate to momentarily slow the blood flow to the arm to measure blood pressure. Once the measurement is collected the cuff will be removed. To measure heart rate, a monitor will be worn around your child's chest. This is an elastic belt that fits all. An area will be designated to allow your child to attach this in private.

Pulse Wave Velocity - in order to measure the health of your child's arteries we will measure the speed at which blood pressure waves flow. In order to measure this we will need to apply a cuff around the neck and upper leg of your child. This measurement should only last 5 minutes.

\section{What are the possible disadvantages of taking part?}

There aren't any significant risks or discomforts within the study. If your child follows our instructions which will ensure that they are appropriately warm for the activity then the risks will be minimised. There is a small risk of injury from the activity (as in any Physical Education class); however there will be trained first aiders on hand to deal with any injuries which may occur. Asthmatics taking part in the study may succumb to exercise induced asthma after the exercise is complete; this is the main reason for the warm down as if this does occur it will be roughly 7 minutes after the main part of the programme. If this does occur we have personnel trained in asthma care in this situation that would be able to support the child appropriately with inhaler use.

\section{What are the possible benefits of taking part?}

Your child may find it interesting to know what some of their measurements are e.g. their lung functions, fitness, physical fitness and mental wellbeing including your child's quality of life could improve through completing this program. The study is also likely to help provide crucial answers to research questions in the health and exercise area of study namely asthma, childhood obesity and other effects of inactivity.

\section{Will my child taking part in the study be kept confidential?}

Although your child's details will be taken prior to conducting the study, they will remain anonymous and this would be the same should the study be published. Your child's personal information will be stored on a protected computer with a password and personal information will be destroyed at the study end (with consent forms stored in a locked cabinet in a secure area, to be destroyed within 5 years of study end). Members of the university will be able to access your information under supervision.

\section{What if I have any questions?}

If you have any problems or need any further information about the study or the testing then please contact any of the research team using the contact details provided. If you have concerns or questions regarding the programme but do not want to contact the research team directly, please contact your child's school tutor. 


\section{Appendix G - Parental/guardian information sheet (control)}

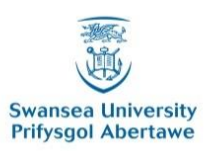

Applied Sports Technology Exercise and Medicine Research Centre (A-STEM) Sport and Health Portfolio, College of Engineering

PARENTAL/GUARDIAN INFORMATION SHEET (Control)

(Version 1.2, Date: 01/06/2015)

Project Title:

The $x 4 a$ trial: eXercise for Asthma with Commando Joes.

Contact Details:

William Eddolls

Charles Winn

Dr Kelly Mackintosh

Dr Melitta McNarry

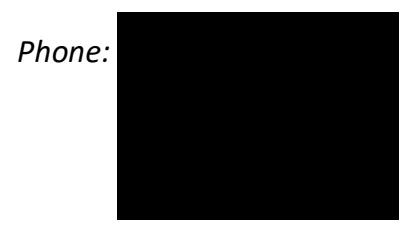

Email:

1. Invitation Paragraph

Thank you for taking the time to read this information sheet, it will provide you with the details of our study and hopefully provide you with the information you require to help you decide if you want your child to participate. It is important to say at this point that the decision to take part is entirely up to you and that your child will not be at a disadvantage for future studies should you decide for them not to participate.

2. What is the purpose of the study?

A link has been shown between exercise and obesity. Worryingly only a small number of children meet the government guidelines of 60 minutes of exercise. This, a lot of the time, is because time does not allow children to get the exercise they need. High intensity exercise has been shown to be a good way of getting enough exercise. So, we want to see whether high intensity training can be used to improve mental and physical health including asthma and quality of life.

3. Why has your child been chosen?

Your child's school has been chosen randomly to be a control school. This will mean that similar measures are made on the children as in the intervention schools where the exercise programme is being delivered so that we can tell if the programme has made a difference. However this does not mean your child has to take part in the study. This is voluntary and you have the right to withdraw them from the study at any time. If you wish to withdraw your child from the study you will not be hassled, and you will not have to give a reason for their withdrawal. Your child will also be able to withdraw themselves from the study at any point without fear of penalty on withdrawal.

4. What will happen to your child if they take part?

Your child will be invited to an assessment day where a number of tests will be conducted. These assessment days will occur at 5 time points across approximately 12 months but should only require your child to miss half a day of lessons per time point. These tests will include: 
Questionnaires - These will include quality of life, mental well-being, asthma health utilization and a physical self-perception questionnaire.

Lung function test and fractional exhaled nitric oxide - Participants will be asked to blow hard into device and gently into a tube connected to a monitor, these will be taken 5 times throughout the year.

Maturation assessments - The children will be asked to complete a questionnaire. It consists of showing line drawings of different pubertal stages. A box next to each picture is then ticked by the child to identify which pubertal stage resembles them. This will be done in complete privacy and only looked at by researchers on completion of the study. Please see attached sheet for line drawings.

Multi-stage fitness test - Your child will be asked to complete a bleep test to assess their fitness at different points throughout the year. It will require them to run $20 \mathrm{~m}$ shuttle runs to the sound of beeps which increase in speed until they no longer reach the line before the beep sounds.

Physical activity measurement - Children will be asked to wear a physical activity monitor for 7 consecutive days. They will also be asked to keep an activity log to record when they remove the accelerometer. Handing out and collecting the accelerometers will take approximately 1 hour at 5 time points over 12 months, totaling 5 hours.

Your child may also be selected for the laboratory testing (roughly 16 per school [8 asthmatic and 8 non-asthmatic]). They will be asked to attend a laboratory session over 4 days (this will be a maximum of 40 mins each session) at 5 time points. These also can be run in your school. During this laboratory testing the children will be asked to complete a number of tests that will include:

Functional fitness test - In this test they will practice using the static bicycle until they are happy with it. It will start off easy and get harder until your child says they can't keep going. During this, they will breath into a mask to allow us to measure the air that they breathe in and out. This mask does not make breathing any harder and you can talk through it and remove it at any time they feel uncomfortable about wearing it. The exercise will last approximately 15 minutes.

Blood analysis - A small pin prick will be administered to the children's finger where an extremely small drop of blood will be taken, if your child does not feel comfortable doing this test then they will be able to decline without any question.

Blood pressure and heart rate - The children will be asked to place an inflatable cuff around their arm. This will inflate to momentarily slow the blood flow to the arm to measure blood pressure. Once the measurement is collected the cuff will be removed. To measure heart rate, a monitor will be worn around your child's chest. This is an elastic belt that fits all. An area will be designated to allow your child to attach this in private.

Pulse Wave Velocity - in order to measure the health of your child's arteries we will measure the speed at which blood pressure waves flow. In order to measure this we will need to apply a cuff around the neck and upper leg of your child. This measurement should only last 5 minutes. 


\section{What are the possible disadvantages of taking part?}

There aren't any significant risks or discomforts within the study. If your child follows our instructions which will ensure that they are appropriately warm for the activity then the risks will be minimised. There is a small risk of injury from the activity (as in any Physical Education class); however there will be trained first aiders on hand to deal with any injuries which may occur. Asthmatics taking part in the study may succumb to exercise induced asthma after the exercise is complete; this is the main reason for the warm down as if this does occur it will be roughly 7 minutes after the main part of the programme. If this does occur we have personnel trained in asthma care in this situation that would be able to support the child appropriately with inhaler use.

\section{What are the possible benefits of taking part?}

Your child may find it interesting to know what some of their measurements are e.g. their lung functions, fitness, physical fitness and mental wellbeing including your child's quality of life. The study is also likely to help provide crucial answers to research questions in the health and exercise area of study namely asthma, childhood obesity and other effects of inactivity.

\section{Will my child taking part in the study be kept confidential?}

Although your child's details will be taken prior to conducting the study, they will remain anonymous and this would be the same should the study be published. Your child's personal information will be stored on a protected computer with a password and personal information will be destroyed at the study end (with consent forms stored in a locked cabinet in a secure area, to be destroyed within 5 years of study end). Members of the university will be able to access your information under supervision.

\section{What if I have any questions?}

If you have any problems or need any further information about the study or the testing then please contact any of the research team using the contact details provided. If you have concerns or questions regarding the programme but do not want to contact the research team directly, please contact your child's school tutor. 


\section{Appendix H - Parental/guardian consent form}

Applied Sports Technology Exercise and Medicine Research Centre (A-STEM)

Sport and Health Portfolio, College of Engineering

Swansea University

Prifysgol Abertawe

\section{PARENTAL/GUARDIAN CONSENT FORM \\ (Version 1.2, Date: 01/06/2015)}

Project Title:

The x4a trial: eXercise for Asthma with Commando Joes.

Contact Details:

William Eddolls

Charles Winn

Dr Kelly Mackintosh

Dr Melitta McNarry

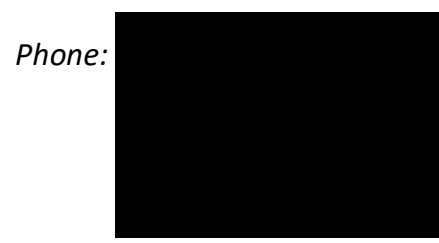

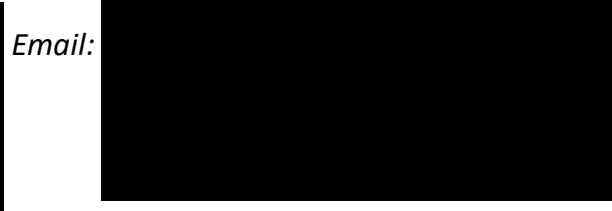

Please initial box

1. I confirm that I have read and understood the information sheet dated 01/06/2015 (version number 1.2) for the above study and have had the opportunity to ask questions.

2. I understand that my participation is voluntary and that I am free to withdraw at any time, without giving any reason, without my legal rights being affected.

3. I understand that sections of any of data obtained may be looked at by responsible individuals from the Swansea University or from regulatory authorities where it is relevant to my taking part in research. I give permission for these individuals to have access to these records. Analysis will be done on anonymous data.

4. I give consent for video and voice recording (discussion groups) and Photography.

5. Does your child have asthma, as confirmed by a doctor?

Yes/No

6. I agree for my child to take part in the above study.

Name of Child

Name of Parent/Guardian

Date

Signature

Researcher

Date

Signature 


\section{Appendix I - Participant information sheet (intervention)}

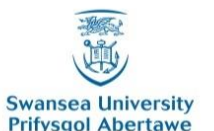

Prifysgol Abertawe

Applied Sports Technology Exercise and Medicine Research Centre (A-STEM)

Sport and Health Portfolio, College of Engineering

\section{PARTICIPANT'S INFORMATION SHEET (Intervention)}

(Version 1.2, Date: 01/06/2015)

Project Title:

The $x 4 a$ trial: eXercise for Asthma with Commando Joes.

\section{Contact Details:}

William Eddolls

Charles Winn

Dr Kelly Mackintosh

Dr Melitta McNarry

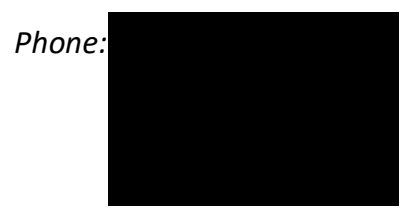

Email:

1. Invitation Paragraph

You are being invited to take part in a research study. Before you decide it is important for you to understand why the research is being done and what it will involve. Please take time to read the following information carefully and discuss it with others if you wish. Ask us if there is anything that is not clear or if you would like more information. If you want to stop taking part in this testing at anytime just tell one of the researchers.

\section{What is the purpose of the study?}

A link has been shown between obesity and lack of exercise. Worryingly only a few children meet guidelines that suggest that children and teenagers get 60 minutes of exercise per day. This study is looking into exercise and how it can affect health both mentally and physically in secondary school children in the South Wales. This is so that we can try and improve mental and physical health of children your age across Wales and the U.K.

\section{Why have I been chosen?}

You been chosen because you fit the example this study wishes to look at. You are between the ages of 11-14 years.

However this does not mean you have to take part in the study. Meaning you can withdraw from the study at any time without fear of penalty on withdrawal. If you want to withdraw you will not have to give a reason. Just let one of us know.

\section{What will happen to if you take part?}

Your will be invited to attend our exercise program. This is a 45 minute exercise session that includes 30 minutes of high intensity exercise conducted 3 times a week before or after school over 6 months. Over the course of the intervention this totals approximately 54 hours. These sessions will alter weekly. An example session would be planned as follows: 
Warm-up - This will last approximately 5 minutes. In the warm-up you will take part in light jogging and stretches to ensure their body is ready to exercise.

High intensity exercise - This will consist of numerous bouts of 30 second exercises followed by 30 seconds rest. Exercises will include numerous military style games. This section of the programme will last 30 minutes.

Warm-down - This will be conducted to ensure that you experience minimal muscle soreness following exercise. This will last 10 minutes to allow for possible asthma related issues.

You will be invited to an assessment day where a number of tests will be conducted. These assessment days will occur at 5 time points across approximately 12 months but should only require you to miss half a day of lessons per time point. These tests will include:

Questionnaires - These will include quality of life, mental well-being, asthma health utilization, asthma control and an anxiety questionnaire.

Static Bicycle

Lung function test and fractional exhaled nitric oxide - You will be asked to blow hard into one device and gently into a tube connected to a monitor, these will be taken 5 times throughout the year.

Maturation assessments - You will be asked to complete a questionnaire. It consists of showing line drawings of different pubertal stages. A box next to each picture is then ticked by yourself to identify which pubertal stage resembles you. This will be done in complete privacy and only looked at by researchers on completion of the study. Please see attached sheet for line drawings.

Multi-stage fitness test - You will be asked to complete a bleep test to assess their fitness at different points throughout the year. It will require you to run $20 \mathrm{~m}$ shuttle runs to the sound of beeps which increase in speed until you can no longer reach the line before the beep sounds.

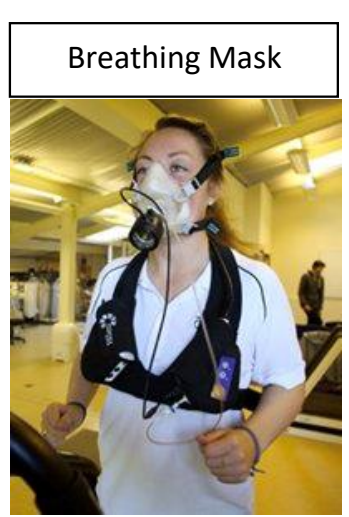

Focus groups - You will be interview in groups of 4-6 and asked questions related to exercise and asthma. These sessions will be either videoed or voice recorded. Those that are randomly selected to attend will be asked to provide us with 1 hour of their time.

Physical activity measurement - You will be asked to wear a physical activity monitor for 7 days. You will also be asked to keep an activity log to record when they remove the monitor. Handing out and collecting the accelerometers will take approximately 1 hour at 5 time points over 12 months, totaling 5 hours.

You may also be selected for the laboratory testing (roughly 16 per school 8 asthmatic and 8 nonasthmatic]). You will be asked to attend a laboratory session over 4 days (this will be a maximum of 40 mins each session) at 5 time points. These also can be run in your school. In these laboratory tests you will be asked to complete a number of tests that will include:

Functional fitness test - In this test you will practice using the static bicycle until you are happy with 
it. It will start off easy and get harder until your say you can't keep going. During this, you will breath into a mask to allow us to measure the air that you breathe in and out. This mask does not make breathing any harder and you can talk through it and remove it at any time you feel uncomfortable about wearing it. The exercise will last approximately 15 minutes.

Blood analysis - A small pin prick will be administered to your finger where an extremely small drop of blood will be taken, if you do not feel comfortable doing this test then you will be able to decline without any question.

Blood pressure and heart rate - You will be asked to place an inflatable cuff around your arm. This will inflate to momentarily slow the blood flow to the arm to measure blood pressure. Once the measurement is collected the cuff will be removed. To measure heart rate, a monitor will be worn around your chest. This is an elastic belt that fits all. An area will be designated to allow you to attach this in private.

Pulse Wave Velocity - in order to measure the health of your arteries we will measure the speed at which blood pressure waves flow. In order to measure this we will need to apply a cuff around your neck and upper leg. This measurement should only last 5 minutes.

\section{What are the possible disadvantages of taking part?}

There aren't any real risks or discomforts within the study. If you follow our instructions which will ensure that you are warmed up for the activity then the risks will be reduced. There is a small risk of injury from the activity (as in any Physical Education class); however there will be trained first aiders on hand to deal with any injuries which may occur. Asthmatics taking part in the study may succumb to exercise induced asthma after the exercise is complete. However this is unlikely. This is the main reason for the warm down as if this does occur it will be roughly 7 minutes after the main part of the programme. If this does occur we have trained personnel who will be able to care for you.

\section{What are the possible benefits of taking part?}

You may find it interesting to know what some of your measurements are e.g. your lung functions, fitness. Physical fitness and mental wellbeing including your quality of life could improve through completing this program. The study is also likely to help provide crucial answers to research questions in the health and exercise area of study namely asthma, childhood obesity and other effects of inactivity.

\section{Will my taking part in the study be kept confidential?}

Yes. Although your details will be collected before the start of the study, you will remain nameless and this will be the same if they study is published. Your personal information will be stored on a protected computer with a password. Only members of the university will be able to access your information under strict supervision.

\section{What if I have any questions?}

If you have any problems or need any further information about the study or the testing then please contact Charlie or Tom on the two first addresses at the start of the pack. If you have concerns or questions regarding the programme but do not want to contact the research team directly, please contact your school tutor. 


\section{Appendix J - Participant information sheet (control)}

Applied Sports Technology Exercise and Medicine Research Centre (A-STEM)

Sport and Health Portfolio, College of Engineering

\section{PARTICIPANT'S INFORMATION SHEET (Control) \\ (Version 1.2, Date: 01/06/2015)}

Project Title:

The x4a trial: eXercise for Asthma with Commando Joes.

\section{Contact Details:}

William Eddolls

Charles Winn

Dr Kelly Mackintosh

Dr Melitta McNarry

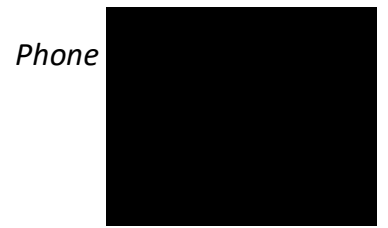

Email:

1. Invitation Paragraph

You are being invited to take part in a research study. Before you decide it is important for you to understand why the research is being done and what it will involve. Please take time to read the following information carefully and discuss it with others if you wish. Ask us if there is anything that is not clear or if you would like more information. If you want to stop taking part in this testing at anytime just tell one of the researchers.

\section{What is the purpose of the study?}

A link has been shown between obesity and lack of exercise. Worryingly only a few children meet guidelines that suggest that children and teenagers get 60 minutes of exercise per day. This study is looking into exercise and how it can affect health both mentally and physically in secondary school children in the South Wales. This is so that we can try and improve mental and physical health of children your age across Wales and the U.K.

\section{Why have I been chosen?}

You been chosen because you fit the example this study wishes to look at. You are between the ages of 11-14 years.

However this does not mean you have to take part in the study. Meaning you can withdraw from the study at any time without fear of penalty on withdrawal. If you want to withdraw you will not have to give a reason. Just let one of us know.

\section{What will happen to if you take part?}

You will be invited to an assessment day where a number of tests will be conducted. These assessment days will occur at 5 time points across approximately 12 months but should only require you to miss half a day of lessons per time point. These tests will include: 
Questionnaires - These will include quality of life, mental well-being, asthma health utilization, asthma control and an anxiety questionnaire.

Lung function test and fractional exhaled nitric oxide - You will be asked to blow hard into one device and gently into a tube connected to a monitor, these will be taken 5 times throughout the year.

Multi Stage Fitness Test - You will be asked to complete a bleep test to assess their fitness at different points throughout the year. It will require you to run $20 \mathrm{~m}$ shuttle runs to the sound of beeps which increase in speed until you can no longer reach the line before the beep sounds.

Maturation assessments - You will be asked to complete a questionnaire. It consists of showing line drawings of different pubertal stages. A box next to

Static Cycle
each picture is then ticked by yourself to identify which pubertal stage resembles you. This will be done in complete privacy and only looked at by researchers on completion of the study. Please see attached sheet for line drawings.

Physical activity measurement - You will be asked to wear a physical activity monitor for 7 days. You will also be asked to keep an activity log to record when they remove the monitor. Handing out and collecting the accelerometers will take approximately

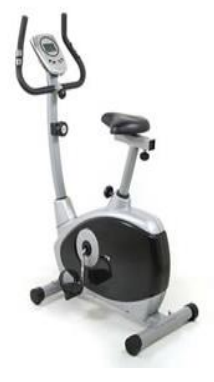
1 hour at 5 time points over 12 months, totaling 5 hours.

You may also be selected for the laboratory testing (roughly 16 per school 8 asthmatic and 8 nonasthmatic]). You will be asked to attend a laboratory session over 4 days (this will be a maximum of 40 mins each session) at 5 time points. These also can be run in your school. In these laboratory tests you will be asked to complete a number of tests that will include:

\section{Breathing Mask}

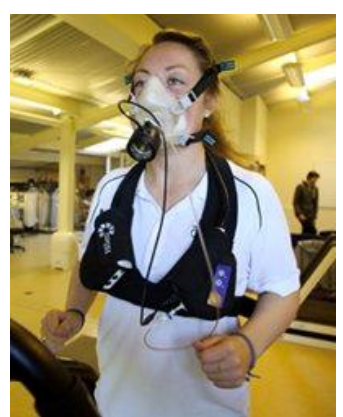

Functional fitness test - In this test you will practice using the static bicycle until you are happy with it. It will start off easy and get harder until your say you can't keep going. During this, you will breath into a mask to allow us to measure the air that you breathe in and out. This mask does not make breathing any harder and you can talk through it and remove it at any time you feel uncomfortable about wearing it. The exercise will last approximately 15 minutes.

Blood analysis - A small pin prick will be administered to your finger where an extremely small drop of blood will be taken, if you do not feel comfortable doing this test then you will be able to decline without any question.

Blood pressure and heart rate - You will be asked to place an inflatable cuff around your arm. This will inflate to momentarily slow the blood flow to the arm to measure blood pressure. Once the measurement is collected the cuff will be removed. To measure heart rate, a monitor will be worn 
around your chest. This is an elastic belt that fits all. An area will be designated to allow you to attach this in private.

Pulse Wave Velocity - in order to measure the health of your arteries we will measure the speed at which blood pressure waves flow. In order to measure this we will need to apply a cuff around your neck and upper leg. This measurement should only last 5 minutes.

\section{What are the possible disadvantages of taking part?}

There aren't any real risks or discomforts within the study. If you follow our instructions which will ensure that you are warmed up for the activity then the risks will be reduced. There is a small risk of injury from the activity (as in any Physical Education class); however there will be trained first aiders on hand to deal with any injuries which may occur. Asthmatics taking part in the study may succumb to exercise induced asthma after the exercise is complete. However this is unlikely. This is the main reason for the warm down as if this does occur it will be roughly 7 minutes after the main part of the programme. If this does occur we have trained personnel who will be able to care for you.

\section{What are the possible benefits of taking part?}

You may find it interesting to know what some of your measurements are e.g. your lung functions, fitness. The study is also likely to help provide crucial answers to research questions in the health and exercise area of study namely asthma, childhood obesity and other effects of inactivity.

\section{Will my taking part in the study be kept confidential?}

Yes. Although your details will be collected before the start of the study, you will remain nameless and this will be the same if they study is published. Your personal information will be stored on a protected computer with a password. Only members of the university will be able to access your information under strict supervision.

\section{What if I have any questions?}

If you have any problems or need any further information about the study or the testing then please contact Charlie or Tom on the two first addresses at the start of the pack. If you have concerns or questions regarding the programme but do not want to contact the research team directly, please contact your school tutor. 


\section{Appendix K - Participant assent form}

Applied Sports Technology Exercise and Medicine Research Centre (A-STEM)

Sport and Health Portfolio, College of Engineering

\section{PARTICIPANT'S ASSENT FORM}

(Version 1.2, Date: 01/06/2015)

Project Title:

The x4a trial: eXercise for Asthma with Commando Joes.

Contact Details:

William Eddolls

Charles Winn

Dr Kelly Mackintosh

Dr Melitta McNarry
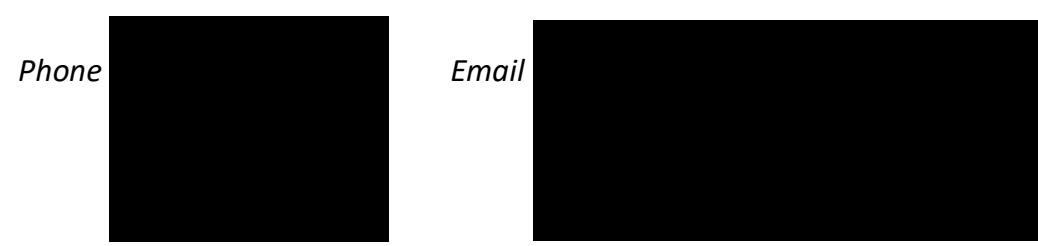

Please initial box

1. I confirm that I have read and understood the information sheet dated 01/06/2105 (version number 1.2) for the above study and have had the opportunity to ask questions.

2. I understand that my participation is voluntary and that I am free to withdraw at any time, without giving any reason, without my medical care or legal rights being affected.

3. I understand that sections of any of data obtained may be looked at by responsible individuals from the Swansea University or from regulatory authorities where it is relevant to my taking part in research. I give permission for these individuals to have access to these records. Analysis will be done on anonymous data.

4. I give consent for video and voice recording and photography.

5. Do you have asthma, as confirmed by a doctor?

6. I agree to take part in the above study.

Name of Participant

$$
\text { Date }
$$

Signature
Yes/No
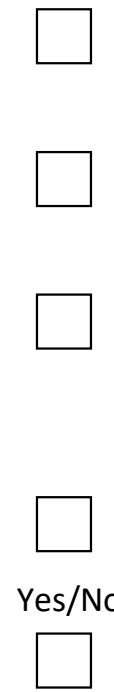
Appendix L - Self-report Tanner stage scale (male)

$\checkmark$ Tick the box that most closely resembles yourself.
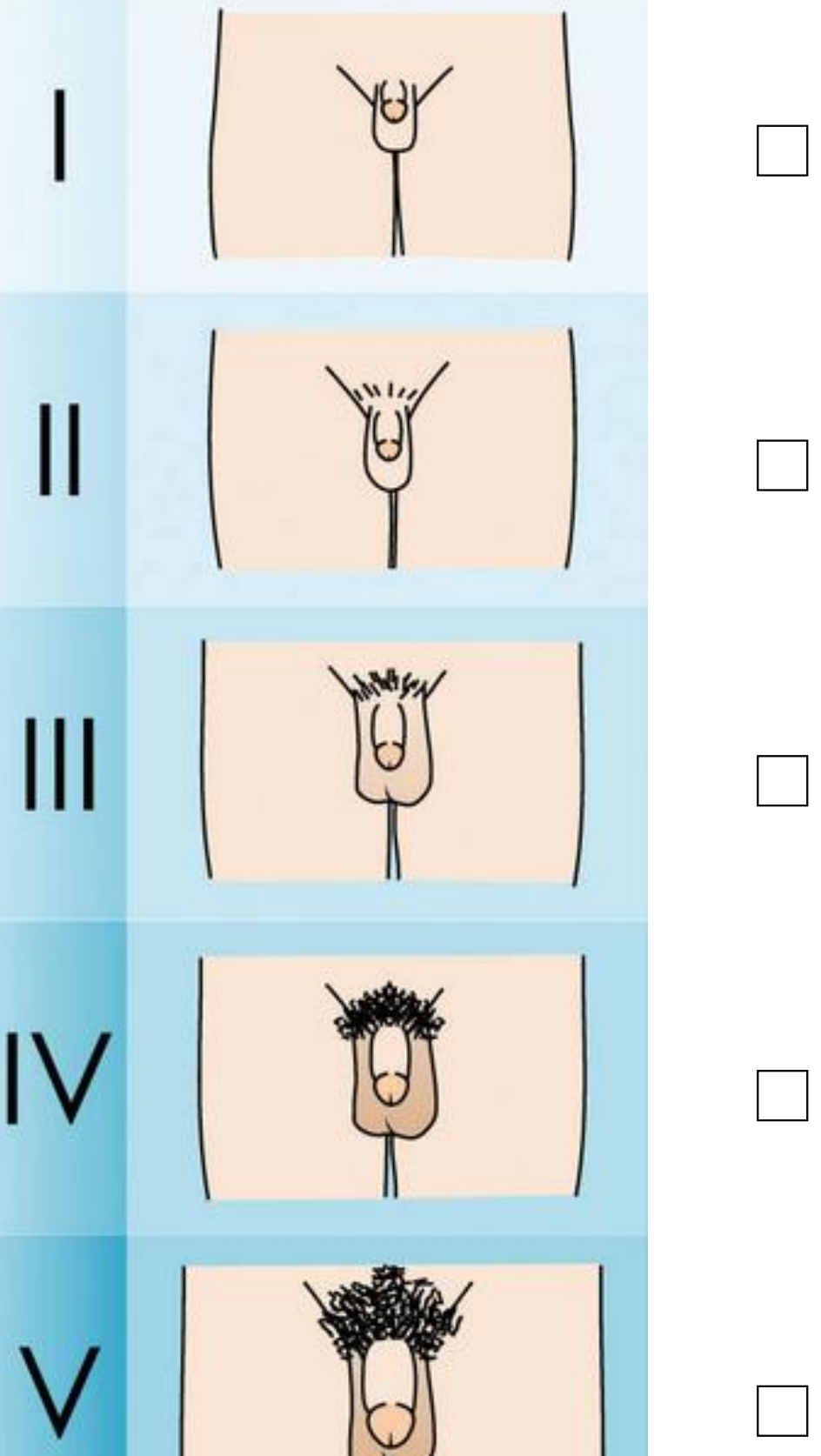
Appendix M - Self-report Tanner stage scale (female)

$\square$ Tick the box that most closely resembles yourself.

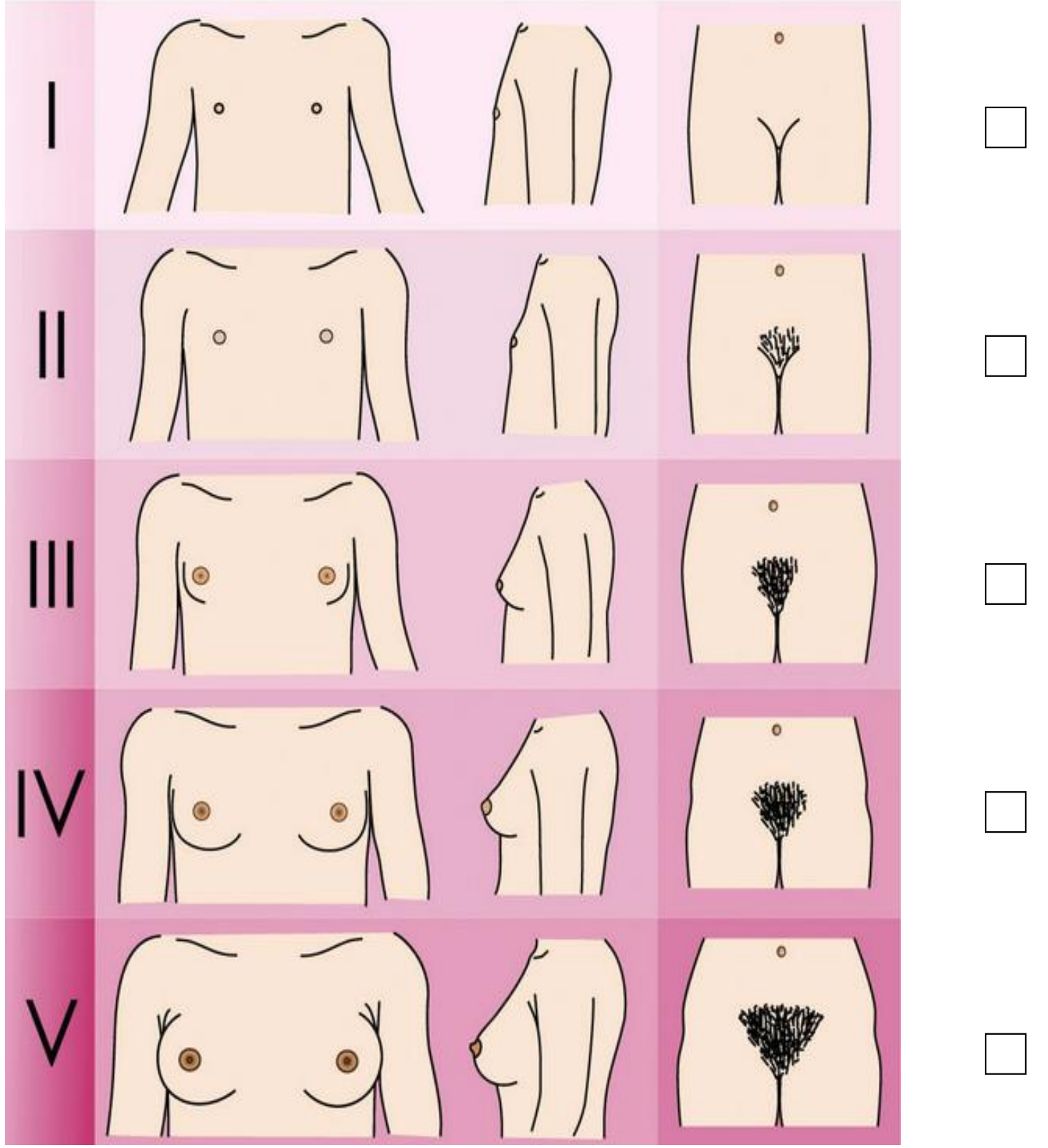




\section{Appendix N - Centre for Epidemiological Studies Depression Scale for Children INSTRUCTIONS}

Below is a list of the ways you might have felt or acted. Please check how much you have felt this way during the past week.

\section{DURING THE PAST WEEK}

1. I was bothered by things that usually don't bother me.

2. I did not feel like eating, I wasn't very hungry.

3. I wasn't able to feel happy, even when my family or friends tried to help me feel better.

4. I felt like I was just as good as other kids.

5. I felt like I couldn't pay attention to what I was doing.

\section{DURING THE PAST WEEK}

6. I felt down and unhappy.

7. I felt like I was too tired to do things.

8. I felt like something good was going to happen.

9. I felt like things I did before didn't work out right.

10. I felt scared.

\section{DURING THE PAST WEEK}

11. I didn't sleep as well as I usually sleep.

12. I was happy

13. I was more quiet than usual.

14. I felt lonely, like I didn't have any friends.

15. I felt like kids I know were not friendly or that they didn't want to be with me.

\section{DURING THE PAST WEEK}

16. I had a good time.

17. I felt like crying.

18. I felt sad.

19. I felt people didn't like me

20. It was hard to get started doing things.

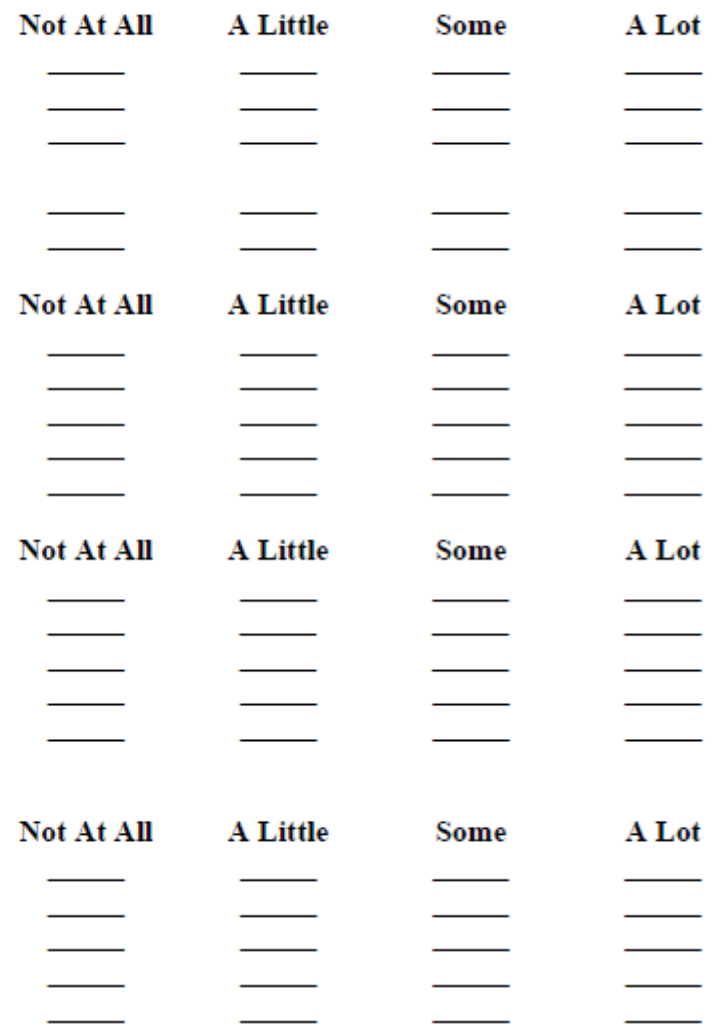


Appendix O - Pediatric Quality of Life Inventory

In the PAST MONTH, how much of a problem has this been for you ...

\begin{tabular}{|l|c|c|c|c|c|}
\hline About My HEALtH AND Activities (problems with...) & Never & $\begin{array}{c}\text { Almost } \\
\text { Never }\end{array}$ & $\begin{array}{c}\text { Somne- } \\
\text { times }\end{array}$ & Often & $\begin{array}{c}\text { Slmost } \\
\text { Allways }\end{array}$ \\
\hline $\begin{array}{l}\text { 1. It is hard for me to walk more than a couple of streets } \\
\text { (about } 100 \text { metres) }\end{array}$ & 0 & 1 & 2 & 3 & 4 \\
\hline 2. It is hard for me to run & 0 & 1 & 2 & 3 & 4 \\
\hline 3. It is hard for me to do sports activities or exercise & 0 & 1 & 2 & 3 & 4 \\
\hline 4. It is hard for me to lift heavy things & 0 & 1 & 2 & 3 & 4 \\
\hline 5. It is hard for me to have a bath or shower by myself & 0 & 1 & 2 & 3 & 4 \\
\hline 6. It is hard for me to do chores around the house & 0 & 1 & 2 & 3 & 4 \\
\hline 7. I have aches and pains & 0 & 1 & 2 & 3 & 4 \\
\hline 8. I feel tired & 0 & 1 & 2 & 3 & 4 \\
\hline
\end{tabular}

\begin{tabular}{|l|c|c|c|c|c|}
\hline About MY FeELINGs (problems with...) & Never & $\begin{array}{c}\text { Almost } \\
\text { Never }\end{array}$ & $\begin{array}{c}\text { Some- } \\
\text { times }\end{array}$ & $\begin{array}{c}\text { Orten } \\
\text { Almost } \\
\text { Always }\end{array}$ \\
\hline 1. I feel afraid or scared & 0 & 1 & 2 & 3 & 4 \\
\hline 2. I feel sad & 0 & 1 & 2 & 3 & 4 \\
\hline 3. I feel angry & 0 & 1 & 2 & 3 & 4 \\
\hline 4. I have trouble sleeping & 0 & 1 & 2 & 3 & 4 \\
\hline 5. I worry about what will happen to me & 0 & 1 & 2 & 3 & 4 \\
\hline
\end{tabular}

\begin{tabular}{|l|c|c|c|c|c|}
\hline How I Get ON with OTHERs (problems with...) & Never & $\begin{array}{c}\text { Almost } \\
\text { Never }\end{array}$ & $\begin{array}{c}\text { Some- } \\
\text { times }\end{array}$ & Often & $\begin{array}{c}\text { Almost } \\
\text { Always }\end{array}$ \\
\hline 1. I have trouble getting on with other teenagers & 0 & 1 & 2 & 3 & 4 \\
\hline 2. Other teenagers do not want to be my friend & 0 & 1 & 2 & 3 & 4 \\
\hline 3. Other teenagers tease me & 0 & 1 & 2 & 3 & 4 \\
\hline 4. I cannot do things that other teenagers my age can do & 0 & 1 & 2 & 3 & 4 \\
\hline 5. It is hard to keep up with other teenagers my age & 0 & 1 & 2 & 3 & 4 \\
\hline
\end{tabular}

\begin{tabular}{|l|c|c|c|c|c|}
\hline About School / College (problems with...) & Never & $\begin{array}{c}\text { Almost } \\
\text { Never }\end{array}$ & $\begin{array}{c}\text { Some- } \\
\text { times }\end{array}$ & Often & $\begin{array}{c}\text { Almoet } \\
\text { Always }\end{array}$ \\
\hline 1. It is hard to pay attention in class & 0 & 1 & 2 & 3 & 4 \\
\hline 2. I forget things & 0 & 1 & 2 & 3 & 4 \\
\hline $\begin{array}{l}\text { 3. I have trouble keeping up with my school / college } \\
\text { work }\end{array}$ & 0 & 1 & 2 & 3 & 4 \\
\hline 4. I miss school / college because of not feeling well & 0 & 1 & 2 & 3 & 4 \\
\hline 5. I miss school / college to go to the doctor or hospital & 0 & 1 & 2 & 3 & 4 \\
\hline
\end{tabular}


Appendix P - Winn, C. O. N., Mackintosh, K. A., Eddolls, W. T. B., Stratton, G., Wilson, A. M., Rance, J. Y., ... Davies, G. A. (2017). Perceptions of asthma and exercise in adolescents with and without asthma. Journal of Asthma.

Perceptions of asthma and exercise in adolescents with and without asthma 


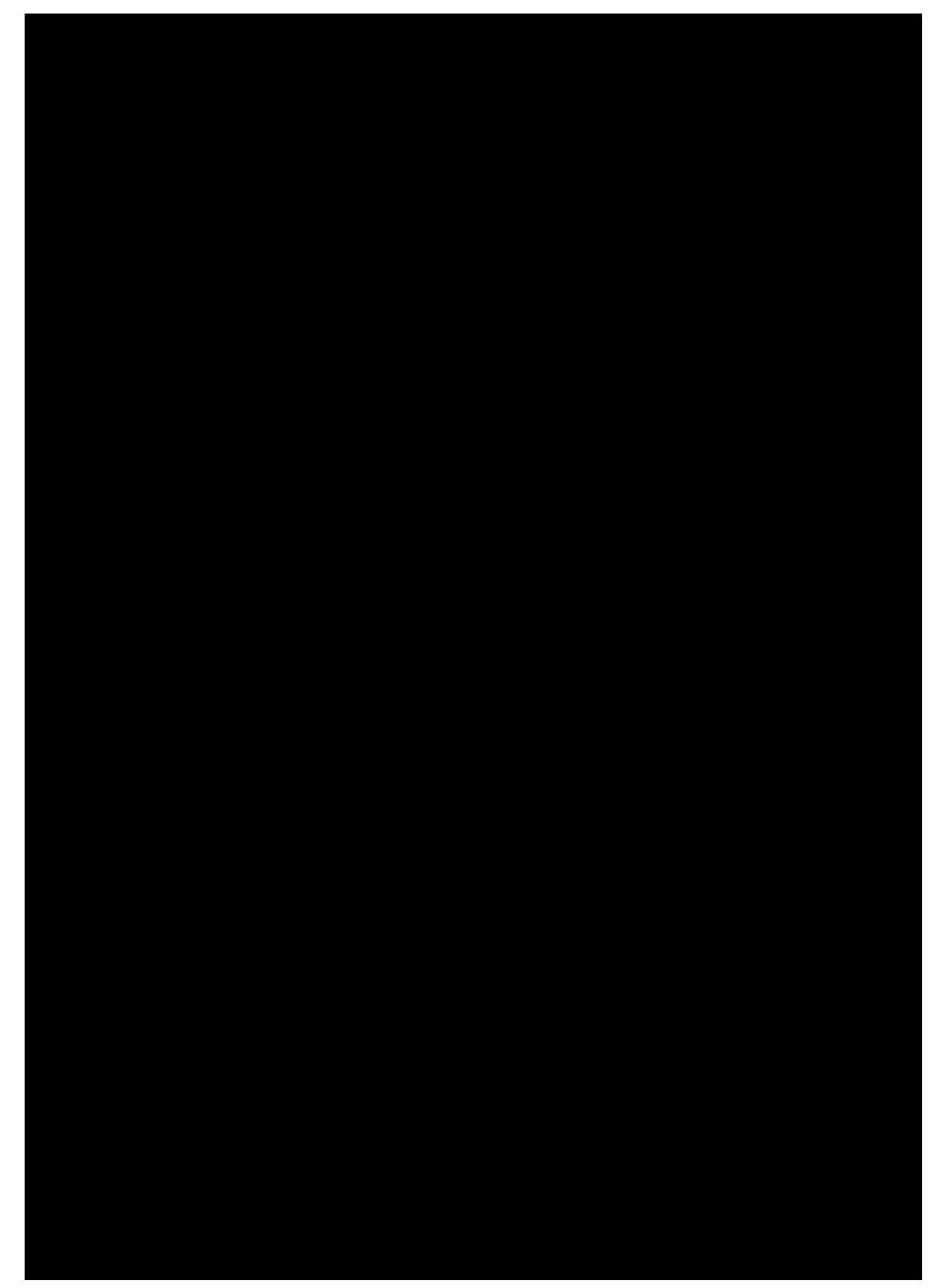


JOUEKAL OF ASTHMA $\Theta 3$

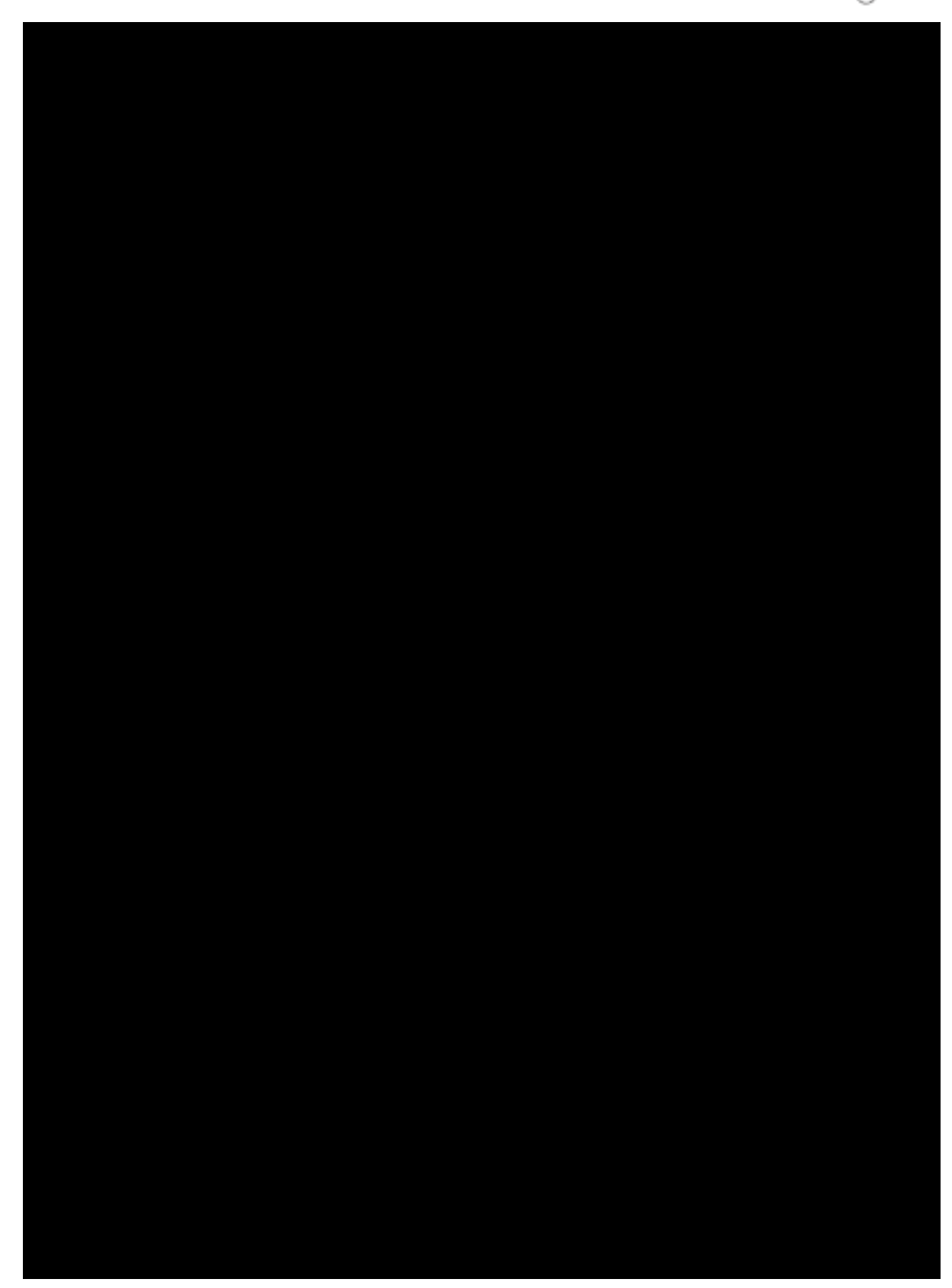


$4 \odot$ CaN. WNWETAL

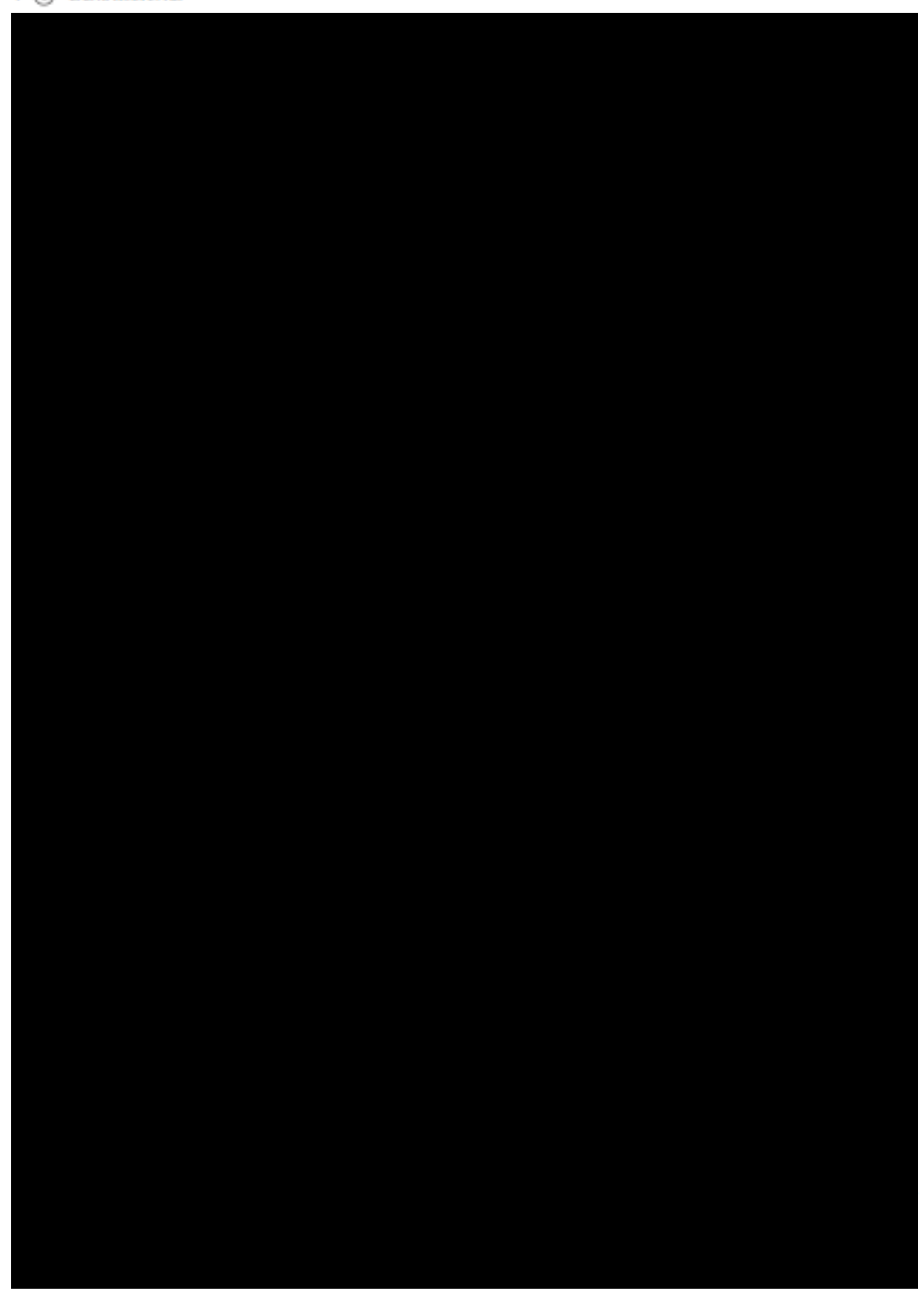




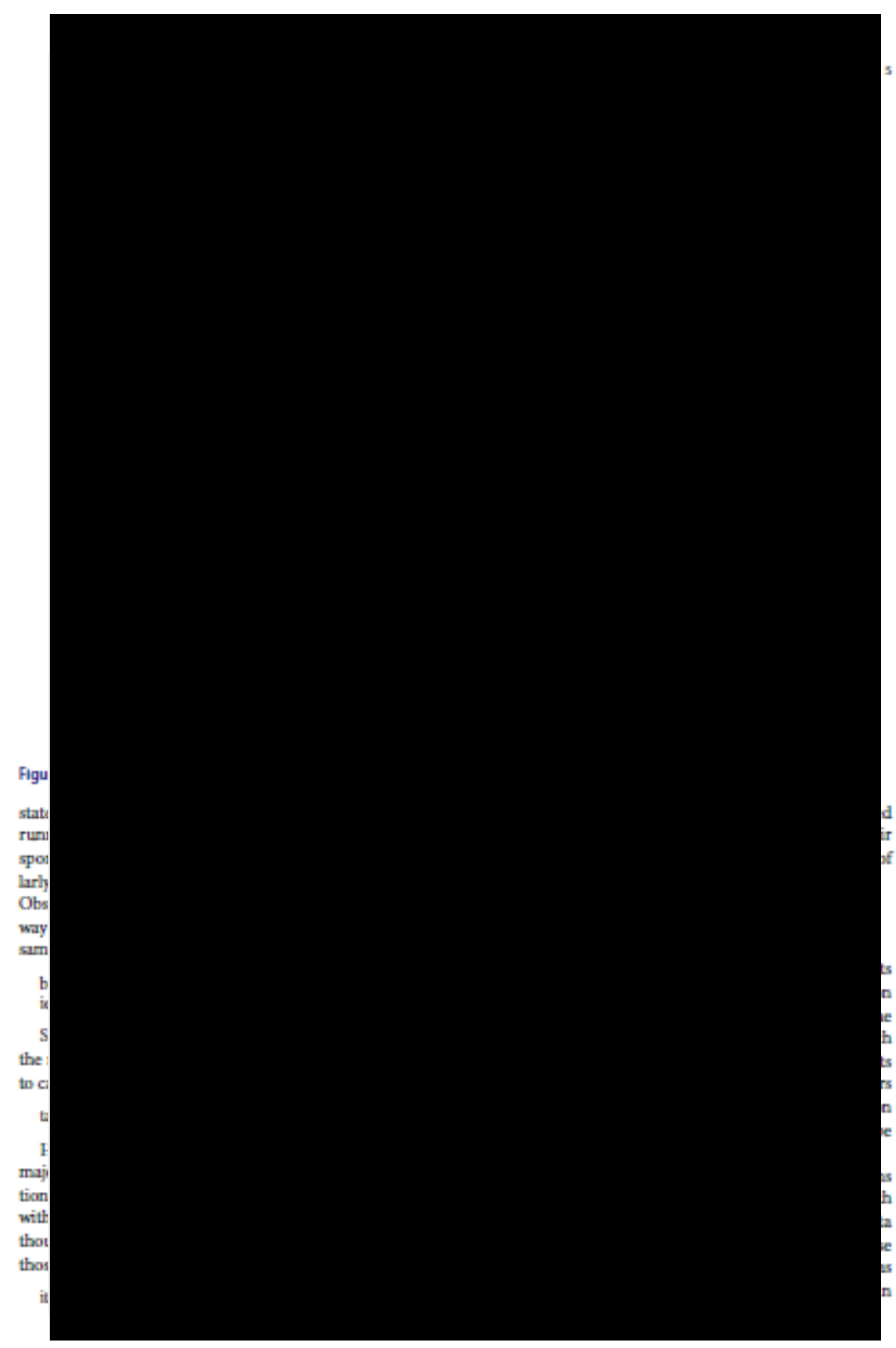




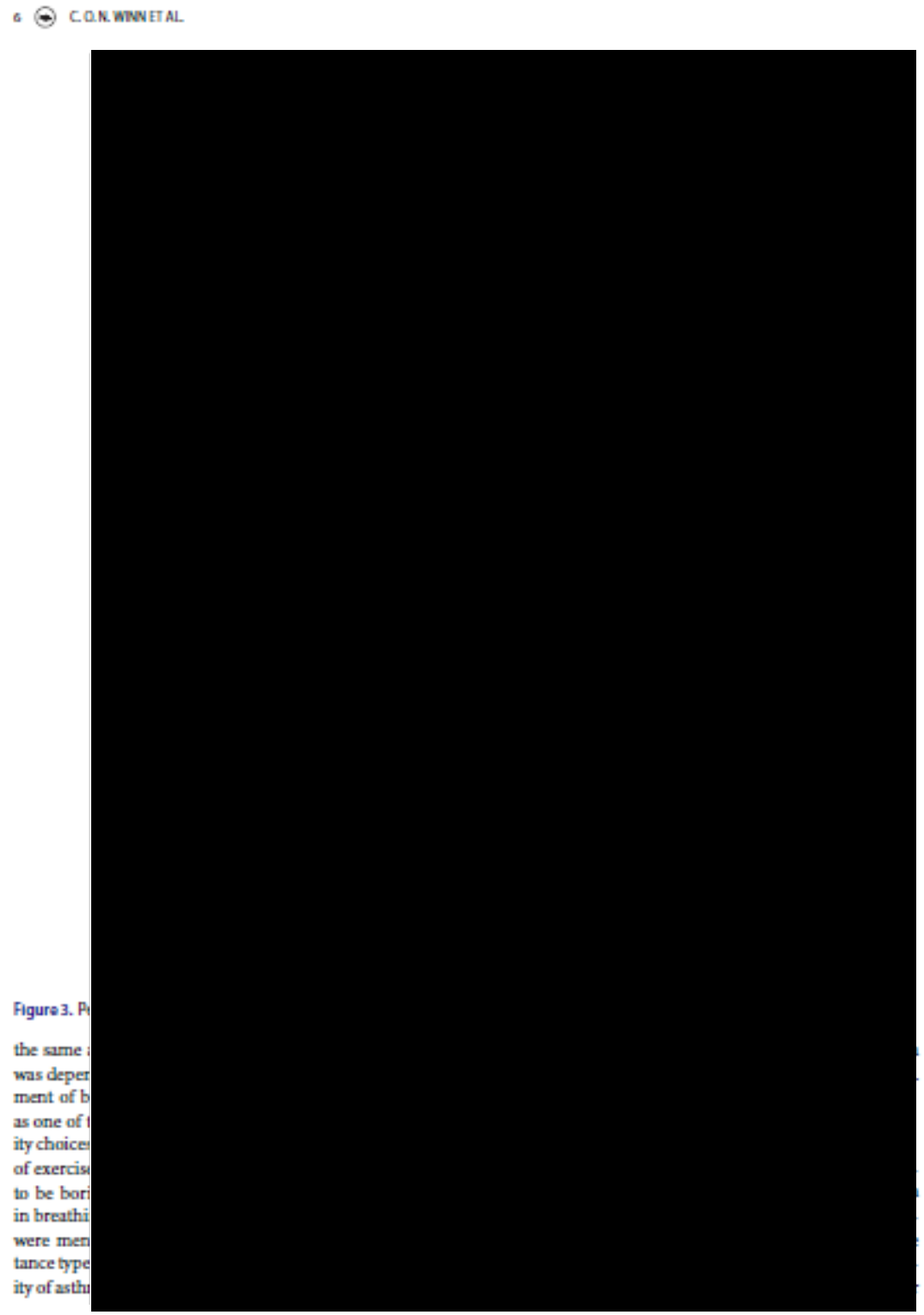


JOUENAL OF ASTHMA $\odot$

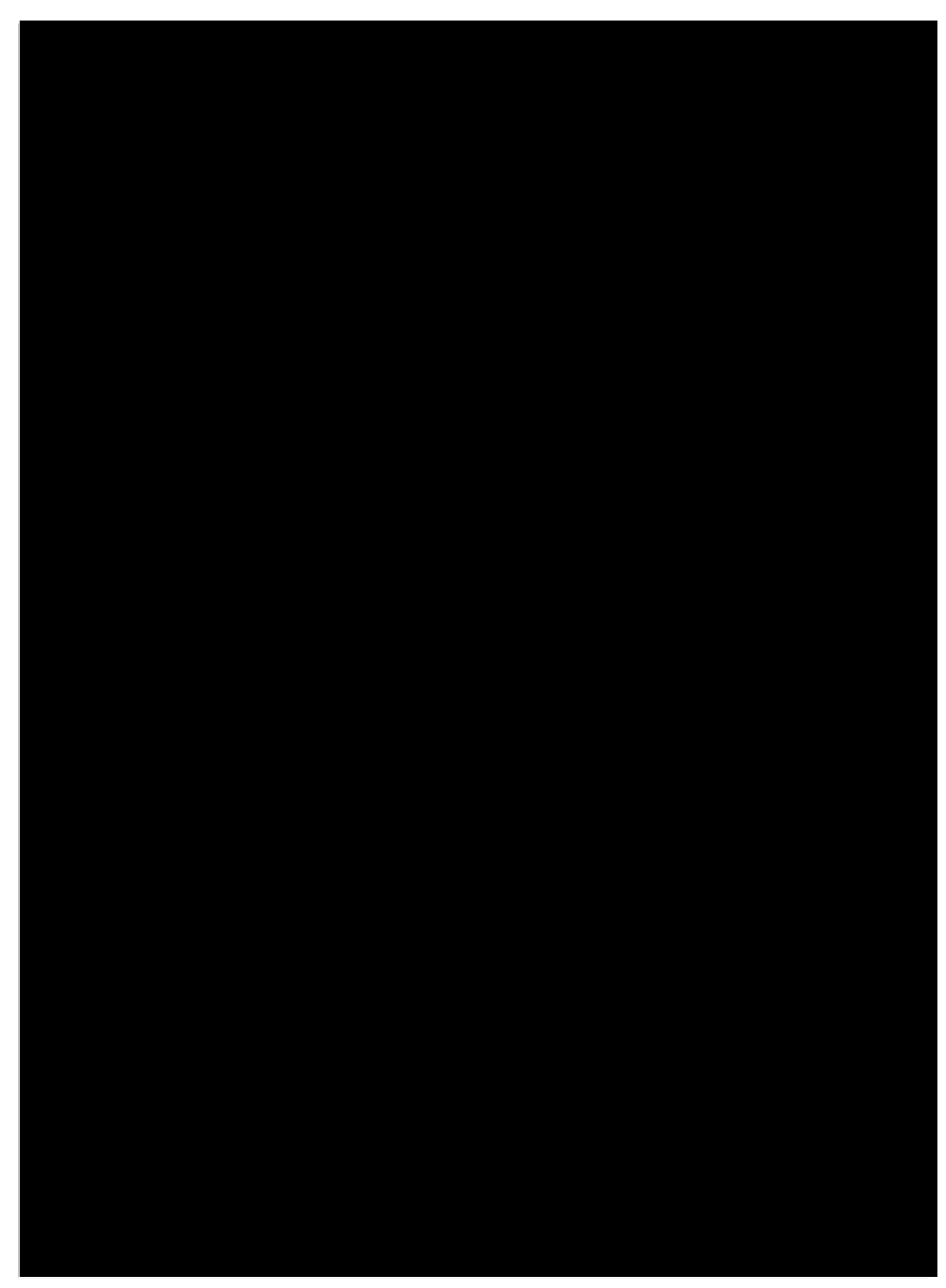




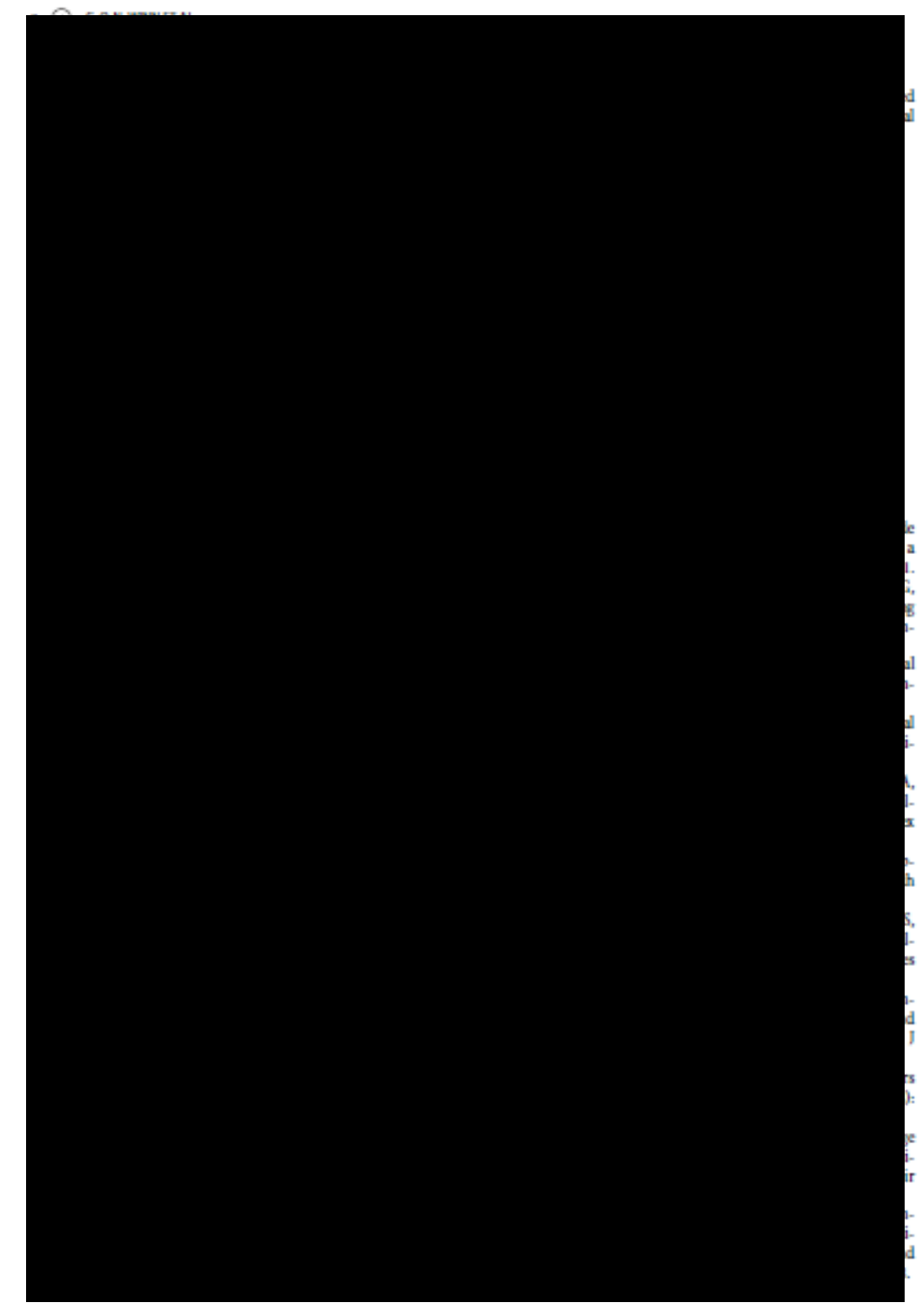


JOURWL OF ASTHM $\odot$

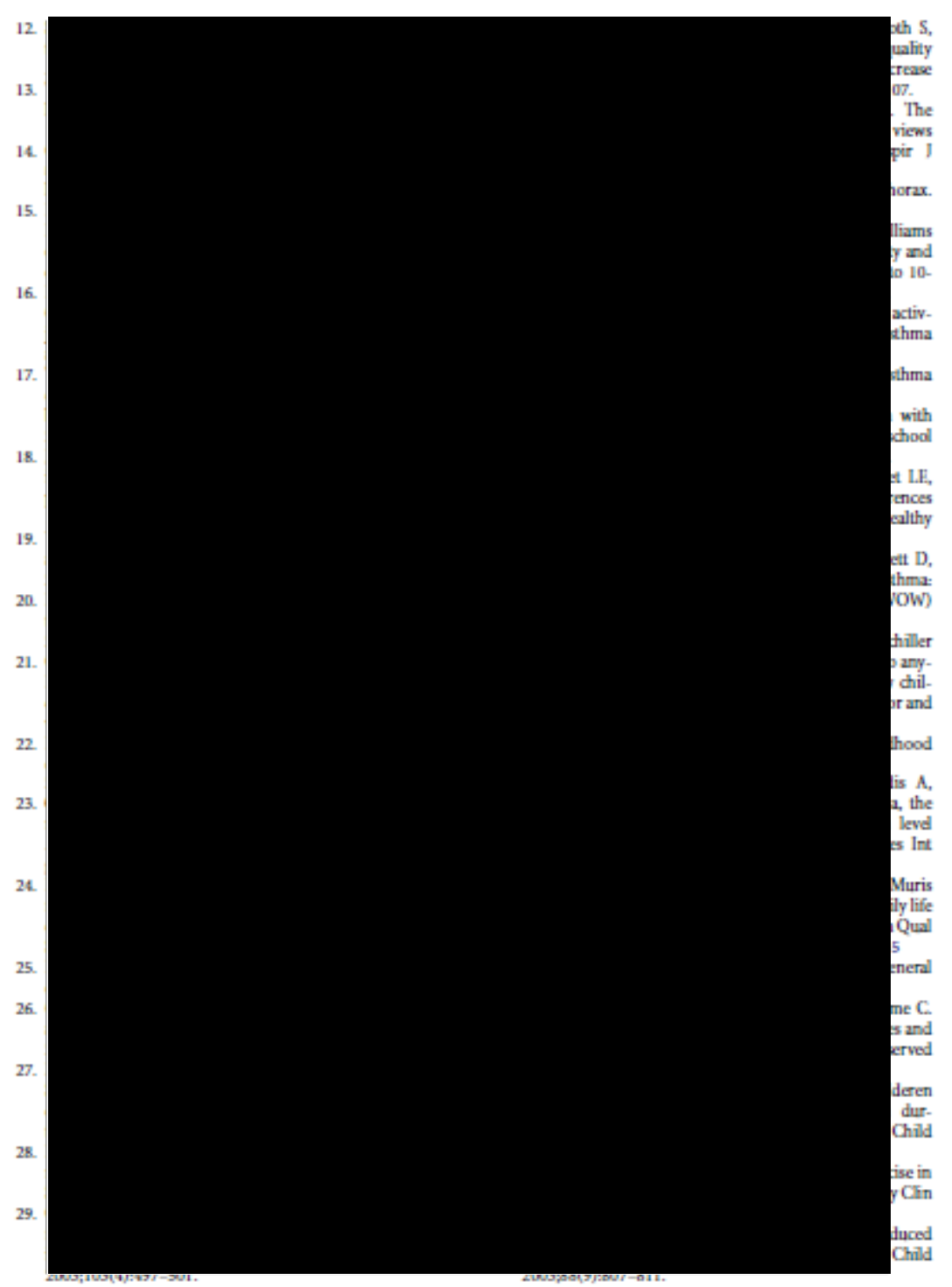




\section{Appendix Q - Generalized Anxiety Disorder 7 questionnaire}

Over the last 2 weeks, how often have you

been bothered by the following problems?

(Use " $"$ " to indicate your answer)

1. Feeling nervous, anxious or on edge

2. Not being able to stop or control worrying

3. Worrying too much about different things

4. Trouble relaxing

5. Being so restless that it is hard to sit still

6. Becoming easily annoyed or irritable

7. Feeling afraid as if something awful might happen
Not Several More than Nearly

at all days half the every day

$\begin{array}{lll}0 & 1 & 2\end{array}$

$\begin{array}{llll}0 & 1 & 2 & 3\end{array}$

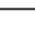

1

23

\begin{tabular}{llll}
\hline 4. Trouble relaxing & 0 & 1 & 2 \\
\hline 5. Being so restless that it is hard to sit still & 0 & 2 & 3 \\
\hline
\end{tabular}

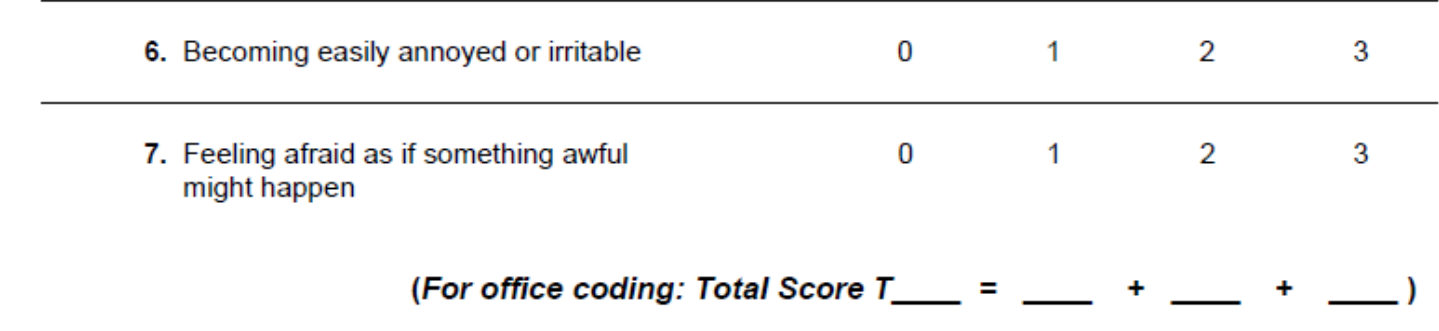




\section{Appendix R - Attendee interview script}

- Ground rules

1. Before you speak state your name

2. One person speak at a time

3. Talk loudly...

4. Speak for yourself, using "I" statements

5. Participate in both talking and listening

6. Be critical of ideas but respect different points of view and different perspectives

7. Stay on the topic and don't digress too much

8. Maintain the confidentiality of opinions expressed in this discussion

9. Focus on issues that need to be discussed and not individuals

10. Wait for one person to finish speaking and don't interrupt others

- Ice Breaker - (we start) Give name and what you most enjoy doing

- What do you think about Commando Joe's?

- What did you think of the Commando Joe's sessions?

- How would you describe the sessions to friends? And what about family?

- Thinking about the sessions that you attended, describe your favourite session...

○ What was it about that session that you enjoyed?

○ What made the session enjoyable?

- And what about your least favourite session, how would you describe that?

- What was it about the session that you didn't like?

- What would have made that particular session more enjoyable?

- Overall, what was the best part of Commando Joe's?

- Overall, what was your least favourite part of Commando Joe's?

\section{Attendance}

- What made you originally want to attend Commando Joe's?

- What did you think it was going to be like before you attended? 
- Now think back to your first session. What was your first impression of the intervention?

- What was your impression of the sessions at the end?

○ Why do you think your impressions changed? OR: How did your initial impressions change over time?

- How often did you attend the sessions?

○ Why did you attend that many sessions?

- What was it that made you want to go?

○ Why?

- What was it that made you not want to go? AND/OR What stopped you being able to attend?

○ Why?

- Why do you think some people signed up but didn't attend?

- How do you think we need to encourage people to attend these sort of sessions in the future?

- What sorts of things do you think we needed to do to make sure people kept attending?

As you know the sessions were run as high intensity interval training...

- Describe what you thought high intensity interval training was before the sessions started...

- Now describe what you think high intensity interval training is now...

- What is your opinion of high intensity interval training?

- What did you enjoy most about it?

○ What did you enjoy least about it?

- How would you describe high intensity interval training to someone else?

- If you were to design a high intensity interval training session what would it be like?

- What would you think about this becoming part of your PE lesson options?

○ Why?

\section{How the sessions made you feel?}


- Think back to just before a session. How did you feel before the session started?

○ Prompt about how they feel mentally and physically

○ Why do you think that is?

- And what about after the session finished. How did you feel then?

- Prompt about how they feel mentally and physically

○ Why do you think that is?

- What do you think the benefits are of attending the sessions?

○ Why?

○ Can they give any real-life examples?

- Do you think you have had any personal benefits to the sessions?

- How have the sessions made you feel?

○ If they talk about tiredness etc. ask about how they felt as a person...

○ Can they give any real-life examples?

- Thinking back to what we have just spoken about, did the sessions changed how you think about Commando Joe's?

○ Did the sessions change how you think about high intensity training?

Moving on again, we are now going to talk about the Commando Joe or $\mathrm{Mr}$ Hudson (Remind them that it is completely anonymous and that what their individual comments won't get back to the Commando Joe):

- What did you think about Mr Hudson?

- What did you think about how he delivered the sessions?

- What did you like the most about Mr Hudson?

- What did you like the least about the Mr Hudson?

- What could he have done to improve the sessions?

- How could he have changed and improved the way he instructed the sessions?

- Describe what the ideal Commando Joe would be like?

- If you could have any one taking the session, who would it be (and why)?

- How would having someone else deliver the sessions change the sessions?

○ Obviously, all of these need to be probed...

- Okay, so one last time, if there was one thing you could change about the session what would you change and why? 


\section{Appendix S - Non-attendee interview script}

- Ground rules

1. Before you speak state your name

2. One person speak at a time

3. Talk loudly...

4. Speak for yourself, using "I" statements

5. Participate in both talking and listening

6. Be critical of ideas but respect different points of view and different perspectives

7. Stay on the topic and don't digress too much

8. Maintain the confidentiality of opinions expressed in this discussion

9. Focus on issues that need to be discussed and not individuals

10. Wait for one person to finish speaking and don't interrupt others

- Ice Breaker - (we start) Give name and what you most enjoy doing

- What do you think about Commando Joe's?

- What did you think the sessions would be like?

\section{(If some attended, ask the following questions)}

- What did you think of the Commando Joe's sessions?

- How would you describe the sessions to friends? And what about family?

- Thinking about the sessions that you attended, describe your favourite session...

○ What was it about that session that you enjoyed?

$\circ$ What made the session enjoyable?

- And what about your least favourite session, how would you describe that?

- What was it about the session that you didn't like?

- What would have made that particular session more enjoyable?

\section{(For those that didn't attend any sessions)}

- What did you think of the Commando Joe's sessions?

- How did people that turned up to the sessions describe them to you? 


\section{Attendance}

- What made you originally want to attend Commando Joe's?

- What did you think it was going to be like?

\section{(If they turned up)}

- How often did you attend the sessions?

○ Why did you attend that many sessions?

- What was your first impression of the intervention? And did that change over time?

- What was it that made you want to go?

○ Why?

- What was it that made you not want to go?

○ Why?

- What was the reason you stopped coming?

- For those that didn't come to a single session, what was it that meant you didn't attend?

- What sort of thing do you think we could have done to encourage you to come to the sessions and why?

- What could we have changed that meant you would have come?

As you may know the sessions were run as high intensity interval training...

- Describe what you think high intensity interval training is...

- What is your opinion of high intensity interval training?

- Do you think that because it was high intensity training it put you off?

○ Why?

○ Was it/did you think it was too hard?

- If you were to design a high intensity interval training session what would it look like?

- What would you think about high intensity interval training becoming part of your PE lesson options?

○ Why? 
- Now forgetting the high intensity training. If we could have done any sort of exercise, what sort of exercise could we have put on that would have encouraged you to turn up to the sessions and why?

Moving on again, we are now going to talk about the Commando Joe or Mr Hudson (Remind them that it is completely anonymous and that what their individual comments won't get back to the Commando Joe):

- What did you think about Mr Hudson?

- What did you like the most about Mr Hudson?

- What did you like the least about the Mr Hudson?

- What could he have done to improve

- Describe what the ideal Commando Joe would be like?

- If you could have any one taking the session, who would it be (and why)?

- How would having someone else deliver the sessions encourage you to come?

○ Obviously, all of these need to be probed... 


\section{Appendix T - Authorship statement}

Candidate William Thompson Bezant Eddolls

Author 1 Melitta Anne McNarry

Author 2 Charles Oliver Nathan Winn

Author 3 Gareth Stratton

Author 4 Kelly Alexandra Mackintosh
College of Engineering

Swansea University

Swansea University

Swansea University

Swansea University

High-intensity interval training interventions in children and adolescents: a systematic review

\section{Located in Chapter 3}

Candidate contributed to the design, data collection, data analysis and writing of the manuscript (50\%).

Author 1 contributed to the design and writing of the manuscript (20\%).

Author 2 contributed to the writing of the manuscript (5\%).

Author 3 contributed to the data analysis and writing of the manuscript $(5 \%)$.

Author 4 contributed to the design and writing of the manuscript (20\%).

We the undersigned agree with the above stated "proportion of work undertaken" for each of the above published peer-reviewed manuscripts contributing to this thesis:

Signed Candidate

Author 1

Author 2

Author 3

Author 4

Author 5

Author 6 